University of Louisville

ThinkIR: The University of Louisville's Institutional Repository

$12-2019$

\title{
Characterization of the impact of prenatal cigarette smoke exposure on age- and sex-specific SIRT1-mediated hepatic molecular phenotype in C57BL/6 mice.
}

\section{Kendall S. Stocke}

University of Louisville

Follow this and additional works at: https://ir.library.louisville.edu/etd

Part of the Maternal and Child Health Commons

\section{Recommended Citation}

Stocke, Kendall S., "Characterization of the impact of prenatal cigarette smoke exposure on age- and sexspecific SIRT1-mediated hepatic molecular phenotype in C57BL/6 mice." (2019). Electronic Theses and Dissertations. Paper 3308.

https://doi.org/10.18297/etd/3308

This Doctoral Dissertation is brought to you for free and open access by ThinkIR: The University of Louisville's Institutional Repository. It has been accepted for inclusion in Electronic Theses and Dissertations by an authorized administrator of ThinkIR: The University of Louisville's Institutional Repository. This title appears here courtesy of the author, who has retained all other copyrights. For more information, please contact thinkir@louisville.edu. 


\title{
CHARACTERIZATION OF THE IMPACT OF PRENATAL CIGARETTE SMOKE EXPOSURE ON AGE- AND SEX-SPECIFIC SIRT1-MEDIATED HEPATIC MOLECULAR PHENOTYPE IN C57BL/6 MICE
}

\author{
By \\ Kendall S. Stocke \\ B.S., Kentucky Wesleyan College, 2008 \\ M.S., University of Louisville, 2013
}

A Dissertation

Submitted to the Faculty of the

School of Public Health and Information Sciences of the University of Louisville

In Partial Fulfillment of the Requirements for the degree of

Doctor of Philosophy in Public Health and Information Sciences

Department of Environmental and Occupational Sciences

University of Louisville

December 2019 



\section{CHARACTERIZATION OF THE IMPACT OF PRENATAL CIGARETTE SMOKE EXPOSURE ON AGE- AND SEX-SPECIFIC SIRT1-MEDIATED HEPATIC MOLECULAR PHENOTYPE IN C57BL/6 MICE}

By

Kendall Stocke

B.S., Kentucky Wesleyan College, 2008

M.S., University of Louisville, 2013

A Dissertation Approved on

November 25, 2019

by the following Dissertation Committee:

Rachel E. Neal, Ph.D., Dissertation Director

Cynthia Corbitt, Ph.D.

M. Michele Pisano, Ph.D.

Robert M. Greene, Ph.D.

Gary W. Hoyle, Ph.D.

Qi Zheng, Ph.D. 


\section{DEDICATION}

This dissertation is dedicated to my grandfather Hubert Whitsitt who taught me the value of hard

work, persistence, and perseverance, especially in the face of great adversity. I would not be the person I am today without him. 


\section{ACKNOWLEDGEMENTS}

The people who have ultimately played a role during my time as a Ph.D. student are numerous. I want to thank my wife Sanaya for the love and support (and nearly infinite patience) she provided to me when it came to my work. I also want to thank my mentor Dr. Rachel Neal for always pushing me to do better, whether it came to my lab work, writing, or making myself the best scientist that I can be. I also want to thank her for allowing me the freedom and independence to investigate and try new approaches to analyzing data. I want to thank the other members of my committee: Dr. Cynthia Corbitt, for always offering her perspective and advice, Drs. Michele Pisano and Robert Greene for the use of their data for part of my analyses and the use of their cigarette smoke exposure instrument; Dr. Gary Hoyle for the continual support and comments on my dissertation work; and Dr. Qi Zheng for his comments and help in guiding my initial statistical analyses. I want to thank Dr. Guy Brock for answering all the random questions that I had when I was starting out learning how to use R for my statistical analyses. In a similar vein, I want to thank Dr. Doug Lorenz for his invaluable advice and guidance on how to implement some of the more complex aspects of my experimental design into my statistical analyses using R. Finally, I want to thank my whole family, both in America and across the world, who always provided me with unconditional love and support. I also want to thank my whole family for always being exceptionally patient when I excitedly and exhaustively speak to them about some aspect of my work. 


\begin{abstract}
CHARACTERIZATION OF THE IMPACT OF PRENATAL CIGARETTE SMOKE EXPOSURE ON AGE- AND SEX-SPECIFIC SIRT1-MEDIATED HEPATIC MOLECULAR PHENOTYPE IN
\end{abstract} C57BL/6 MICE Kendall Stocke

December 2, 2019

In the U.S., $7.2 \%$ of women still report having smoked at some point during pregnancy despite known risks to fetal health. Prenatal cigarette smoke exposure (CSE) in children puts them at risk for low birth weight, premature birth, and other adverse impacts on developmental and postnatal health. Children subjected to prenatal CSE have a higher risk of adulthood metabolic disease predicted by the Barker Hypothesis. The hepatic molecular phenotype associated with this risk is unknown. This dissertation characterizes the prenatal CSE-induced hepatic molecular phenotype during three key life stages.

We used a murine model of prenatal CSE utilizing a Teague TE-10C cigarette smoking apparatus that induces low birth weight with catch-up growth. This model was used in a preliminary study to observe the hepatic metabolic phenotype of prenatal (gestational day 6-18) CSE offspring during adulthood in conjunction with a high-fat diet (HFD) or control low-fat diet (CD) feeding for three months. The hepatic molecular phenotype was characterized by the expression of SIRT1, a Class III deacetylase $\mathrm{NAD}^{+}$sensor, and associated genes and proteins. Female CSE offspring maintained on a HFD exhibited exacerbated weight gain and body fat accumulation while male CSE offspring exhibited decreased SIRT1-related protein expression.

Follow up studies subjected offspring to prenatal (GD1-18) CSE and measured their hepatic molecular phenotype at the neonatal and post-weaning periods. Male CSE offspring at 1.5 weeks of age exhibited signs of elevated SIRT1 signaling independent of the main SIRT1 regulatory loop. Female CSE 1.5-week old offspring exhibited signs of delayed functional liver 
ontogeny via depressed expression of Sirt1, related enzymes, and serum glucose levels. Weaned fed male CSE offspring exhibited mixed expression in SIRT1-regulatory pathways. Fasted male CSE offspring exhibited signs of an exacerbated fasting response via increased SIRT1-related gluconeogenic mRNA expression. Fed female CSE offspring exhibited an exacerbated fed response and untimely gluconeogenic mRNA expression. Fasted female CSE offspring exhibited signs of an exacerbated fasting response via SIRT1-related mRNA and protein expression and untimely lipogenic mRNA expression. This work attempted to extend the Barker Hypothesis by characterizing the hepatic molecular phenotype at key life stages in offspring subjected to prenatal CSE. 


\section{TABLE OF CONTENTS}

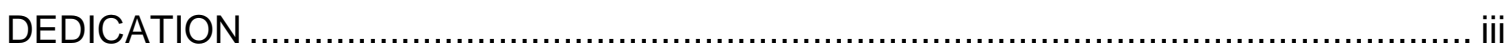

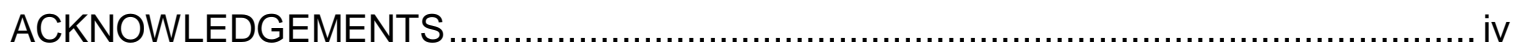

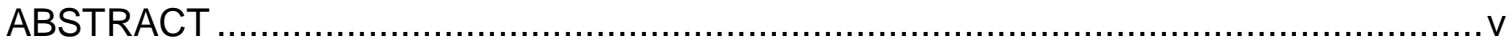

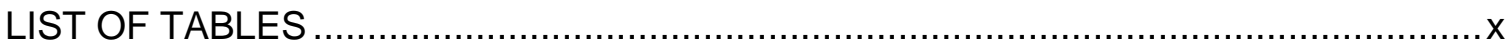

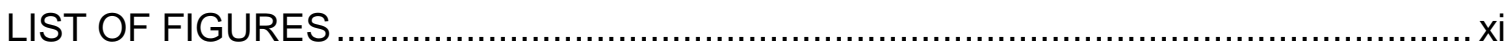

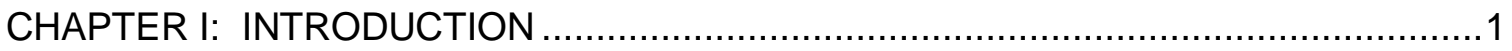

Direct Cigarette Smoke Exposure …...........................................................

Secondhand Cigarette Smoke Exposure ................................................. 4

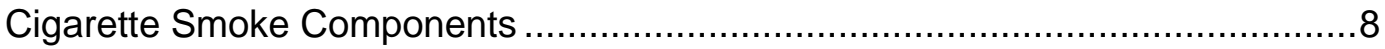

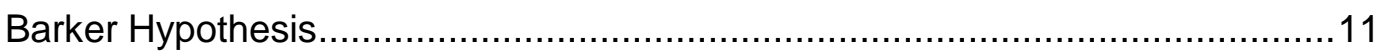

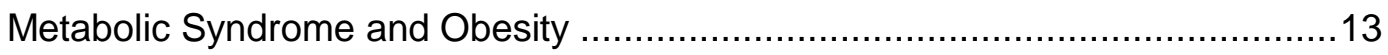

Liver Development and Function................................................................ 15

Non-Alcoholic Fatty Liver Disease .........................................................22

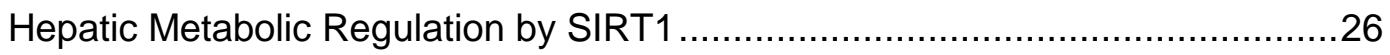

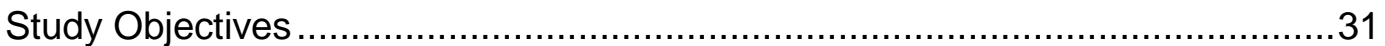


CHAPTER II: PRENATAL CIGARETTE SMOKE EXPOSURE IMPACTS ADULTHOOD WEIGHT GAIN AND SIRT1-ASSOCIATED METABOLIC PATHWAYS DISTINCTLY BY

SEX

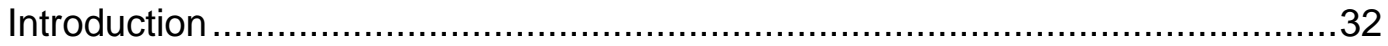

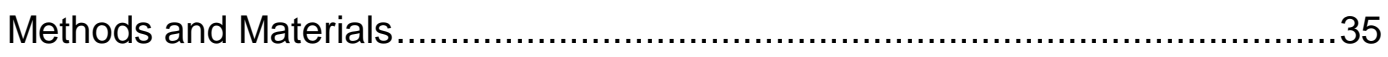

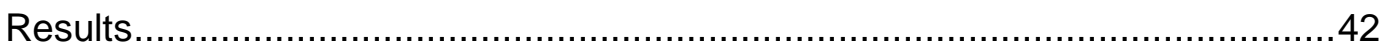

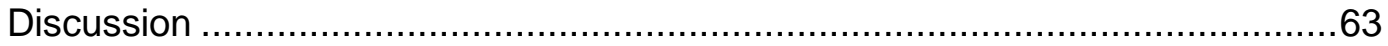

CHAPTER III: IN UTERO CIGARETTE SMOKE EXPOSURE PERTURBS HEPATIC EXPRESSION OF METABOLIC PATHWAYS IN A SEX-DEPENDENT MANNER

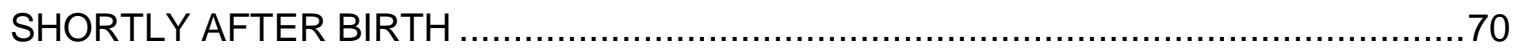

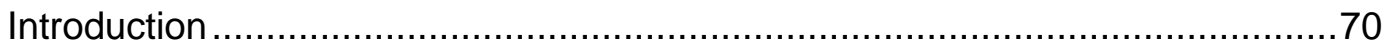

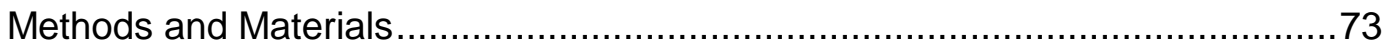

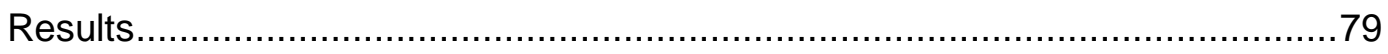

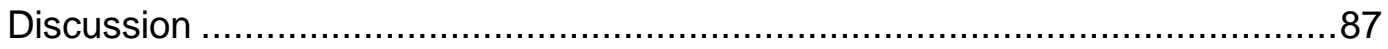

CHAPTER IV: IN UTERO EXPOSURE TO CIGARETTE SMOKE EXACERBATES THE HEPATIC FASTING RESPONSE IN OFFSPRING AND CAUSES POOR ...................93

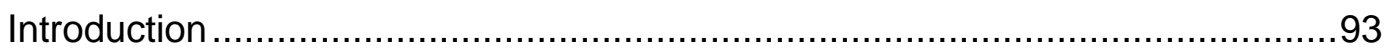

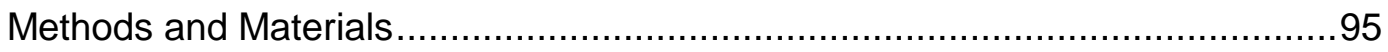

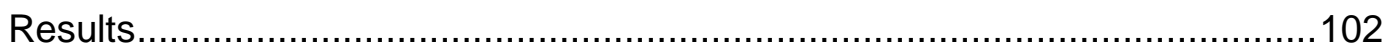

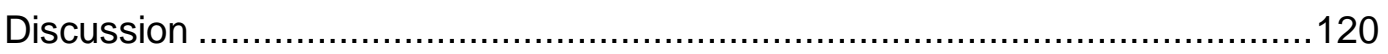

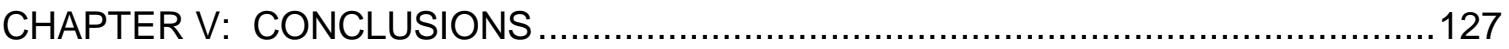


REFERENCES.

CURRICULUM VITAE .. 


\section{LIST OF TABLES}

Table 1. Forward and reverse primer pairs for RT-qPCR ….......................................77

Table 2. Forward and reverse primer pairs for RT-qPCR 100 


\section{LIST OF FIGURES}

Figure 1. SIRT1 Regulatory and Downstream Target Pathways ................................29

Figure 2. CSE exacerbates diet-modulated sex-specific weight gain ..........................43

Figure 3. CSE-induced increase in sex-specific weight and modulated by diet ............45

Figure 4. High-fat diet results in greater calorie consumption ................................47

Figure 5. Minor effect of CSE on the rate of calorie consumption modulated by diet.....48

Figure 6A. Female CSE offspring traveled more quickly on HFD than male CSE

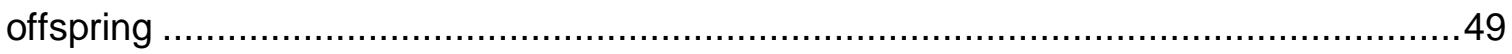

Figure 6B. Female CSE offspring traveled more on HFD than male CSE offspring ......51

Figure 6C. Female CSE offspring on HFD display less anxious behavior than male CSE

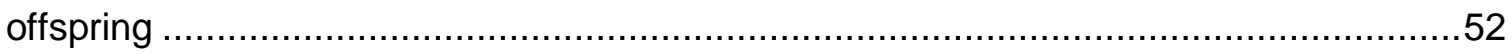

Figure 7. Prenatal CSE increases body fat accumulation in female CSE offspring

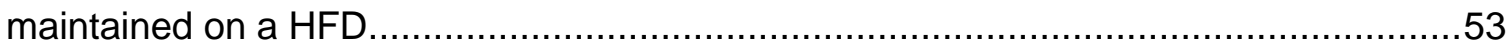

Figure 8. Male offspring maintained on a low-fat diet do not exhibit liver fat deposition in

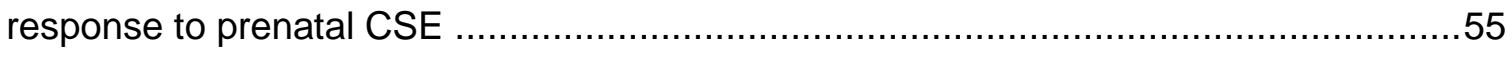

Figure 9A. Prenatal CSE or high-fat diet independently decrease hepatic SIRT1 protein

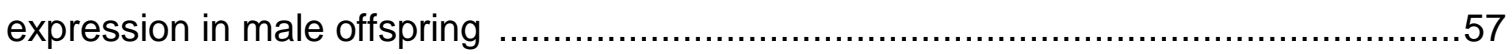

Figure 9B. Prenatal CSE does not impact hepatic NAMPT expression, while HFD increases NAMPT expression in female offspring 
Figure 10A. The impact of prenatal CSE on hepatic GAPDH expression is highly modulated by diet in male offspring

Figure 10B. Prenatal CSE and high-fat diet independently decrease PEPCK expression in male offspring

Figure 11A. Offspring exposed in utero to cigarette smoke exhibit a phenotype of low birth weight followed by subsequent catch-up growth

Figure 11B. Female offspring exposed in utero to cigarette smoke exhibit decreased serum glucose levels

Figure 12. Exposure in utero to cigarette smoke alters hepatic expression of SIRT1 and NAMPT in male offspring and decreases acetylation of lysine residues in all offspring...... 83-84

Figure 13A. Prenatal cigarette smoke exposure in male offspring increased hepatic gene expression of Sirt1, Nampt, and key regulatory gluconeogenic enzymes.....

Figure 13B. Prenatal cigarette smoke exposure in female offspring decreased hepatic expression of SIRT1-regulatory loop genes, as well as related groups of metabolic genes

Figure 14. Offspring exposed in utero to cigarette smoke exhibit catch-up growth by weaning

Figure 15. Exposure in utero to cigarette smoke increased expression of SIRT1 in fed female offspring and decreased hepatic expression of NAMPT in all fed offspring 105-106

Figure 16. Exposure in utero to cigarette smoke altered hepatic expression of SIRT1 and NAMPT in fasted male offspring 107-108 
Figure 17. Prenatal cigarette smoke exposure in fed offspring increased hepatic gene expression of Sirt1 and modulates the expression of other related enzymes by sex

Figure 18. Prenatal cigarette smoke exposure in fasted offspring modulated hepatic expression of SIRT1 regulatory loop based on sex...... $112-113$

Figure 19. Prenatal cigarette smoke exposure in fed offspring differentially modulated hepatic gene expression of SIRT1-related metabolic processes by sex $116-117$

Figure 20. Prenatal cigarette smoke exposure in fasted female offspring exacerbated fasting response-induced hepatic gene expression $118-119$ 


\section{CHAPTER I: INTRODUCTION}

\section{Direct Cigarette Smoke Exposure}

The prevalence of smoking in the U.S. population remains a significant public health issue despite campaigns to minimize or eliminate this behavior. The 2016 smoking prevalence among U.S. adults 18 years or older was $15.5 \%$, with the rate being unequal between men (17.5\%) and women (13.5\%) [1]. The smoking prevalence among age groups varies with the highest being at $18.0 \%$ in middle-aged individuals (45-64 years), followed by $17.6 \%$ in adults ( $25-44$ years), $13.1 \%$ in young adults (18-24 years), and $8.8 \%$ in the elderly (65 years or older) [1]. Alaskan Natives/Native Americans smoke at nearly double the rate of White or Black individuals $(31.8 \%$, $16.6 \%$, and $16.5 \%$ respectively) [1]. The smoking prevalence decreased with higher levels of educational attainment [1]. Of those individuals who held a graduate degree, $4.5 \%$ were smokers [1]. The prevalence of smoking in people with a high school degree was $19.7 \%$, while it was $40.6 \%$ for those who received a GED [1]. This difference in smoking prevalence between equivalent academic achievements suggests there was some other underlying factor that educational attainment alone did not fully capture. The prevalence of smoking in people below the poverty line was $56.5 \%$ higher than in individuals at or above the poverty line [1]. The prevalence of smoking in self-reported disabled adults was $21.2 \%$ versus $14.4 \%$ in non-disabled people [1]. Smoking prevalence was higher for people who exhibited serious psychological distress as rated on the Kessler Scale (35.8\%) as compared to individuals who scored low on this clinical measure $(14.7 \%)$ [1]. It is evident that the prevalence of smoking is not evenly distributed in the U.S. population because certain demographic groups are more likely to engage in this health-adverse behavior.

Smoking occurred at a significant rate in the U.S. in 2008 in both high school (8.1\%) [2] and middle school (1.8\%) [2], although it was not as common as in adults (13.7\%) [3]. The rates of smoking any tobacco product in high school and middle school students have declined since 
2014-2015 [2]. However, there has been a tremendous resurgence in the rate of using any tobacco product between $2017-2018$ in high school students (from $19.6 \%$ to $27.1 \%$ ) and middle school students (from $5.6 \%$ to $7.2 \%$ ) [2]. The prevalence of smoking e-cigarettes or vaping has gone down overall from 2014 to 2018 (from $3.7 \%$ to $3.2 \%$, respectively), although it is projected to increase in the future [4]. This upward trend in the prevalence of smoking e-cigarettes or vaping behaviors is likely due to increased numbers of young adults (18-24 years old) engaging in these behaviors [4]. E-cigarette use is particularly concerning in high school students, in which it has increased from 2011 to 2018 (from 1.5\% to 20.8\%, respectively) [5]. Rates of e-cigarette usage has also increased in middle school students from 2011 to 2018 (from 0.6\% to 4.9\%, respectively) [5]. It is evident that the previously declining rates of smoking in adolescents are exhibiting a resurgence due to increasing rates of e-cigarette smoking or vaping behaviors that will undoubtedly contribute to future health problems.

Smoking is still responsible for numerous deaths in the U.S. population. For the period spanning 2010-2014, approximately 638,500 deaths from lung cancer in the U.S. were attributed to smoking (818,500 deaths if including all cancers) [6]. Between the years 2010-2014, an additional 803,000 deaths from cardiovascular and metabolic disease in the U.S. were attributed to smoking and 565,500 deaths from pulmonary diseases [6]. In $2014,448,865$ U.S. adults aged 35 years or older died due to smoking-related causes [7]. The percent attributable fraction of deaths due to smoking in the same age group of U.S. adults in 2014 was highest in Kentucky (22.1\%) followed by Arkansas (21.5\%), Nevada (21.3\%), Tennessee (21.1\%), West Virginia (20.6\%), Oklahoma (20.2\%), and Missouri (20.1\%) [7]. The relative risk of death from various diseases in adults 55-64 years old was exacerbated due to smoking [6]. For lung cancer, the relative risk of death for smokers versus non-smokers was 19.03 for men and 18.95 for women [6]. The relative risk of smokers versus non-smokers of dying due to COPD was 84.76 for men and 22.58 for women [6]. The relative risk for male and female smokers versus non-smokers dying from stroke was 2.07 and 3.27, respectively [6]. The relative risk of dying from coronary heart disease in male smokers versus non-smokers was 2.99, while the relative risk in female smokers versus non-smokers was 3.25 [6]. The risk of death due to other heart disease was 2.50 and 1.49 times higher in male and 
female smokers vs non-smokers, respectively [6]. Smoking caused a clear majority of deaths from lung cancer and COPD [6], which was unsurprising due to smoking being smoking the primary cause of COPD [6]. Smoking increased the risk of several cancers including lung, liver and colorectal cancer [6]. A report on the 2015 Global Burden of Disease Study found that $11.5 \%$ of global deaths were attributable to smoking [8]. A more recent report found that in 2016, smokingattributable deaths from 1990 to 2016 increased by $20.1 \%$ worldwide [9]. This same report also found that smoking was the second and sixth leading risk factor for deaths in men and women, respectively [9]. Despite ongoing efforts to curb this health-adverse behavior in the U.S. and beyond, it is evident that smoking continues to exert a heavy death toll.

Smoking is a significant risk factor for the development of type 2 diabetes. According to a 2007 meta-analysis utilizing 25 cohort studies, there was a higher risk for Type 2 diabetes in heavy smokers compared to light or former smokers [10]. A more recent meta-analysis in 2015 utilizing 88 prospective studies found that the relative risk for Type 2 diabetes current smokers compared to non-smokers was 1.37 [11]. This same meta-analysis found this increase in risk for Type 2 diabetes was dose-dependent with the relative risk being highest in heavy smokers (1.57) followed by moderate (1.34) and light smokers (1.21) [11]. A meta-analysis published in 2017 utilizing 22 studies found that the pooled relative risk of developing Type 2 diabetes was 1.38 in current smokers versus non-smokers and 1.19 for former smokers versus non-smokers [12]. That same meta-analysis found that smoking-attributable cases of Type 2 diabetes in men and women were $18.8 \%$ and 5.4\%, respectively [12]. A prospective cohort study published in 2010 following Korean adults 30-95 years old found that the risk for outpatient treatment for diabetes was 1.55 times higher for current smokers (20 cigarettes per day or more) compared to non-smokers [13]. That prospective cohort study also found that the effect of smoking on outpatient diabetes treatment risk was lower in women compared to men [13]. Considering that the CDC found diabetes in the U.S. was the seventh leading cause of deaths in 2015 and lead to $\$ 245$ billion in medical expenses in 2012 [14], it is evident that smoking has tremendous potential to negatively impact public health.

Smoking has several adverse reproductive effects such as but not limited to decreased sperm quality $[15,16]$ and decreased probability of getting pregnant or achieving a live birth [17]. 
A cross-sectional study published in 2015 found that women who actively smoked had increased odds for spontaneous abortion (1.16 times higher), stillbirths (1.44 times higher), and ectopic pregnancies (1.43) [18]. In a meta-analysis in 2014 utilizing 98 studies, the risk of miscarriage in mothers who actively smoked during pregnancy was 1.32 times higher compared to non-smokers [19]. A meta-analysis published in 2016 utilizing 142 studies found that the risk of either stillbirth or neonatal death was 1.33 times higher for mothers who were active smokers versus non-smokers [20]. A case-control study using Cameroonian women found that smoking increased the odds of ectopic pregnancy by 2.68 fold. [21]. Impacts of cigarette smoke exposure on the fetus and infants are discussed in the section on secondhand cigarette smoke exposure. It is evident that aiding women in abstaining from smoking is critical to preventing adverse reproductive health outcomes.

Smoking has a substantial cost not just on health, but economically as well. Smoking comprised $8.7 \%$ of aggregated annual healthcare spending in U.S. by the year 2010 [22]. Approximately two-thirds of this cost was being paid by the public through Medicaid, Medicare, and other governmental programs [22]. It was found in a 2018 report that a positive return on investment of $78.4 \%$ on Medicaid plans after four years and $30.6 \%$ on Medicare plans after three years could be generated by utilizing the recommended smoking cessation prescription coverage given by the Affordable Care Act [23]. It is evident that minimizing or eliminating smoking not only has the potential to alleviate public health burdens, but also to recoup financial loses in combating smoking-attributable disease.

\section{Secondhand Cigarette Smoke Exposure}

Secondhand cigarette smoke exposure has a significant effect on health outcomes. Between 2010-2014, secondhand smoke in the U.S. was attributable to 206,420 deaths [6]. One study found that secondhand smoke exposure increased the hazard ratio of dying from cardiovascular disease (1.38), coronary heart disease (1.31), and any circulatory disease (1.28) [24]. A positive dose-response relationship was found between secondhand smoke and death from circulatory disease [24]. It was found that people who lived with a partner who smoked more than 30 cigarettes each day were associated with a hazard ratio of 2.94 for circulatory disease-related 
death [24]. A pooled analysis performed by the International Lung Cancer Consortium utilizing 18 case-control studies demonstrated that the odds of developing any lung cancer from being exposed to any amount of secondhand smoke was 1.31 times higher than never being exposed [25]. Notably, secondhand smoke is associated with 2.11 times higher odds of developing small cell carcinoma compared to non-small cell carcinoma [25]. A meta-analysis utilizing 24 studies demonstrated a higher relative risk due to secondhand smoke exposure for developing COPD (1.66), stroke (1.35), and ischemic heart disease (1.27) [26]. It is evident that not only do smokers present a clear danger to their own health by engaging in cigarette smoking, but they also endanger the health of others. It is clearly in the interest of improving public health to provide assistance and encouragement in aiding smoking cessation efforts.

There are several reproductive health outcomes related to secondhand smoke. A crosssectional analysis found that non-smoking women who were exposed to secondhand smoke had higher odds for spontaneous abortion (1.17 times higher), stillbirths (1.55 times higher), and ectopic pregnancies (1.61) [18]. In a meta-analysis in 2014 utilizing 98 studies, the risk of miscarriage in mothers who actively smoked during pregnancy was 1.11 times higher compared to non-smokers [19]. A study published in 2017 found that non-smoking women who lived with at least two smokers had 1.25 higher odds of experiencing pregnancy loss [27]. It is evident that in order to ensure optimal reproductive outcomes, it is essential to continue informing smokers that their behavior not only impacts their own health but also the health of others around them.

A primary outcome of cigarette smoke exposure during pregnancy is intrauterine growth retardation, which presents itself as low birth weight $[28,29]$. It has been found that prenatal cigarette smoke exposure was associated with increased diagnoses of attention deficit hyperactivity disorder in children [30]. A meta-analysis conducted in 2011 using 172 studies found that in utero cigarette smoke exposure increased the rate of congenital disabilities including orofacial clefts, congenital heart defects, gastroschisis, craniosynostosis, clubfoot, anorectal atresia, musculoskeletal defects, hernias, and undescended testes [31]. It has been found that exposure to cigarette smoke in utero doubled the risk of Sudden Infant Death Syndrome in infants [32]. The Ottawa Prenatal Prospective Study Cohort has shown that male children exposed to 
prenatal cigarette smoke have an earlier onset of puberty as measured by the age at which the voice differentiates and the age of onset of shaving [33]. A prospective cohort study found that female children exposed to cigarette smoke in utero exhibited earlier age of menarche that was exacerbated the more cigarettes smoked per day by their mother [34]. It is without question that failure to prevent expectant mothers from engaging in cigarette smoking results in adverse health consequences for their children.

It has been found that mouse models of prenatal CSE exhibit low birth weight [35-41], decreased crown-rump length [41] as well as decreased somite number [42]. Low birth weight has also been documented in rat models of prenatal CSE [43]. Animal models have found alterations in behavioral development such as reference memory, spatial learning, and decreased anxiety [44]. Mice subjected to in utero cigarette smoke exposure exhibited enhanced airway hyperreactivity, inflammation from pulmonary eosinophils, and airway secretion following stimulation by house dust mite inhalation [45]. One study found that female mice subjected to in utero cigarette smoke exposure exhibited increased body weight and both high- and low-density lipoprotein [46]. That same study also found that male offspring exposed in utero to cigarette smoke and maintained on a high-fat diet for two weeks exhibited increased weight, total cholesterol, and high-density lipoprotein [46]. This data indicates that in utero cigarette smoke exposure sensitizes offspring to the effects of high-fat diet consumption, which can only contribute to the likelihood of developing metabolic disease in adulthood. It is evident that animal models of prenatal cigarette smoke exposure also exhibit adverse health outcomes in offspring.

During 2016 in the U.S., $7.2 \%$ of women reported smoking at any point during pregnancy [47], which was a decrease from the previous rate of $8.4 \%$ overall in 2014 [48] and $9.7 \%$ overall in 2008 [49]. Assuming one child per pregnancy, a rate of smoking during pregnancy of $7.2 \%$ in the U.S. translates to at least 284,000 fetuses that were subjected to prenatal secondhand cigarette smoke in 2016 [47]. Of those women who smoked during pregnancy, the highest prevalence occurred in women 20-24 years old, followed by $15-19$ years old and $25-29$ years old [47]. Native Indians and Alaskan Natives are most at risk of smoking during pregnancy, followed by White, Black, Native Hawaiian/Pacific Islander, Hispanic, and Asian [47]. Those more likely to smoke 
during pregnancy had a high school (or equivalent) degree or less, with the prevalence being lowest for lowest for individuals with at least a Bachelor's degree [47]. Although many states in 2016 had a low smoking prevalence in expectant mothers (5\% or less), states such as Kentucky (18.4\%) and West Virginia (25.1\%) had much higher rates of smoking during pregnancy [47]. These rates are lower than the 2014 rates in Kentucky (20.7\%) and West Virginia (27.1\%) [48]. These rates were even lower still than the 1999 rates in Kentucky (24.5\%) and West Virginia (26.1\%) [50]. The trends in these rates highlight the fact that while in some states the rate has been steadily going down, in other places, the rate has not changed much. Even more alarming is the fact that smoking while pregnant has been underreported, with an analysis of 1999-2006 NHANES data indicating that $22.9 \%$ of pregnant women did not report their current smoking status [51]. Women who smoked while pregnant in the U.S. in 1983 incurred an estimated $\$ 267$ million in neonatal healthcare costs [52]. It has been estimated that smoking during pregnancy in the U.S. in 1996 was attributed to \$244 million of neonatal expenses in Medicaid programs[53]. Using U.S. data from 2001-2002, an estimated $\$ 122$ million in costs for delivery of all infants were attributable to maternal smoking during pregnancy [54]. It is evident that the prevalence of smoking during pregnancy is not evenly distributed in the U.S. population because certain demographic groups are more likely to engage in this costly health-adverse behavior.

Even maternal secondhand smoke exposure is enough to induce in utero changes to the fetus. One study found that pregnant women exposed to secondhand smoke had 2.71 times higher odds of delivering a low birth weight infant [55]. Another study found that the effects of secondhand smoke on low birth weight may, in part, be due to decreased placenta size [56]. A cross-sectional study published in 2019 found that the children of mothers exposed to secondhand smoke exhibited a birth weight that was, on average, 205.6 grams lower than normal [57]. A case-control study found that fathers who smoked while their partner was pregnant were associated with 1.15 higher odds of having children that developed childhood acute lymphoblastic leukemia [58]. A Hong Kong birth cohort found that secondhand smoke exposure during pregnancy resulted in increased behavioral problems at 11 years old according to the Revised Parent's Rutter Scales, which 
measure conduct, emotional, and inattention/hyperactivity problems [59]. It is essential to ensure that smokers are aware of the impact that their smoking has on children before they are even born.

\section{Cigarette Smoke Components}

According to the U.S. Food and Drug Administration, there are 93 established harmful and potentially harmful constituents in tobacco smoke [60], but one of the most important and addictive is the alkaloid nicotine. The content of nicotine and other alkaloids in tobacco is dependent on how it is processed, the use of specific blends, sun curing, and additive use to alter the $\mathrm{pH}$ of the generated smoke [61]. Nicotine exerts its addictive effects by competitively binding acetylcholine targets, which has several effects including the release of dopamine [62] and $\beta$-opioids [63] along with inhibition of long term GABA secretion [64]. Nicotine has also been shown to increase levels of serotonin acutely while decreasing serotonin levels chronically [65]. Prenatal nicotine exposure also recapitulates key impacts of prenatal smoke exposure. For example, it has been hypothesized that prenatal nicotine exposure induces adrenal alterations to increase the risk of hypoxia-induced Sudden Infant Death Syndrome [66]. Specifically, $6 \mathrm{mg} / \mathrm{kg} /$ day maternal infusion of nicotine through an osmotic minipump from gestational day 5 to parturition found offspring exhibited inhibited catecholamine release in response to hypoxic challenge alterations [66]. Prenatal nicotine injections twice daily with $2 \mathrm{mg} / \mathrm{kg}$ free base nicotine resulted in offspring with non-sex-specific low birth weight and subsequent catch-up growth by postnatal day 10 [67]. Xu et al. [68] demonstrated that injecting pregnant Wistar rats twice daily with $1 \mathrm{mg} / \mathrm{kg}$ nicotine from gestational day ten results in lower offspring body weight one week after birth. The offspring from this same study also exhibited subsequent catch-up growth in addition to the development of metabolic syndrome when exposed to a high-fat diet [68]. That same study did note some differences in timing of the catchup growth, in which it started in female offspring around postnatal week 11 and in male offspring starting around postnatal week 16 [68]. It has also been found that injecting dams with $1 \mathrm{mg} / \mathrm{kg} / \mathrm{day}$ nicotine bitartrate starting two weeks before mating through weaning results in increased offspring serum and lipid triglycerides [69]. Injecting female Wistar rats with $1 \mathrm{mg} / \mathrm{kg} /$ day nicotine during pregnancy through lactation resulted in lost offspring pancreatic beta-cell mass [70]. This same 
study also found in these offspring glucose intolerance without changes in insulin levels or weight [70]. Prenatal nicotine exposure through maternal subcutaneous injection with $1-3 \mathrm{mg} / \mathrm{kg} /$ day nicotine hydrogen tartrate resulted in lower fetal rat weight [71]. This same study also found proportional decreased fetal brain weight, loss of brain cells and histological abnormalities such as mitochondrial swelling and intracytoplasmic edema in the hippocampal CA1 region of the brain [71]. Infusion of pregnant rat dams with $6 \mathrm{mg} / \mathrm{kg} /$ day nicotine hydrogen tartrate via an implanted osmotic pump starting on gestational day 12 and onward inhibited male offspring brain aromatase activity, which plays an important role in rodent developmental brain masculinization [72]. Specifically, this inhibition of brain aromatase activity in exposed male offspring occurred in the preoptic area, hypothalamus, and amygdala, thereby disrupting brain sexual differentiation [72]. We have not noted any similar published findings in female offspring, which do not depend on prenatal aromatase activity for brain sexual differentiation [73]. As we discuss in more detail in a later section, changes to the hypothalamus can have important consequences on hepatic metabolic development. It is evident that not only does prenatal nicotine exposure have generalized systemic impacts on offspring, but in particular results in lower birth weight and catch-up growth with indications of perturbed metabolic health later in life.

Another principal cigarette constituent is tobacco-specific nitrosamines (TSNAs). These compounds vary in concentration based on processing methods mentioned previously [74] as well as the use of nitrogen-containing fertilizer [75]. The TSNA 4-(methylnitrosamino)-1-(3-pyridyl)-1butanone (NNAL) has been found both in the urine [76-78] and in the amniotic fluid of offspring born to mothers who smoked during pregnancy [79]. Nicotine-derived nitrosamine ketone (NNK) has also been detected in the urine of newborn children exposed in utero to cigarette smoke [78]. $\mathrm{N}$-nitrosamines, of which TSNAs are a part, have known carcinogenic effects. Specifically, NNK and N-nitrosonornicotine (NNN), which are two important TSNAs in tobacco smoke, have been found to cause lung tumors and esophageal tumors, respectively [80]. We did not note at this time any published association of TSNAs with low birth weight or long-term effects such as metabolic syndrome. Given how TSNAs can be transferred to the fetus, it is essential for future studies that 
the potential of TSNAs to induce low birth weight with catch-up growth or adulthood metabolic effects be thoroughly investigated.

Polycyclic aromatic hydrocarbons (PAHs), including benzo[a]pyrene and benzo(a)pyrene diol epoxide, are a class of compounds produced from the incomplete combustion of organic matter [81]. One main factor affecting PAH content in tobacco leaves is the level of nitrates in the soil [82, 83]. The levels of n-nitrosamines and PAHs have been found to be inversely related in tobacco leaves since higher levels of nitrates in the soil lowers the amount of PAHs produced but increases the levels of $n$-nitrosamines [84]. Levels of benzo[a]pyrene (BaP), in particular, can be used as a surrogate for total $\mathrm{PAH}$ levels, as research has indicated that $\mathrm{BaP}$ and total $\mathrm{PAH}$ levels are correlated [85]. The primary mechanism of BaP carcinogenicity is the formation of DNA adducts induced by the metabolic byproduct benzo(a)pyrene diol epoxide (BPDE) [86]. Prenatal exposure to PAHs has been associated with small for gestational age in children $[87,88]$. Prenatal exposure to BaP has been shown to cause low birth weight in children [89]. Another study [90] found that daily oral gavage of pregnant dams with $\mathrm{BaP}$ during gestational day 7-16 resulted in increased obesity-related measures in adult female offspring such as increased weight gain, visceral fat, and hepatic lipid deposition. However, the authors from that study admitted the dosage of BaP they used is 36 -fold higher than what is experienced by women exposed throughout pregnancy to a high amount of PAHs from cigarette smoke or other sources [90]. We did not note any published studies regarding catch-up growth and prenatal exposure to PAHs. It is evident that exposure in utero to PAHs such as BaP manifest some key characteristics predicted by the Barker Hypothesis including low birth weight and increased obesity-related measures in adulthood, but more studies should be done to confirm this is the case.

Volatile organic compounds (VOCs) in cigarettes are produced from the incomplete combustion of organic matter, much like PAHs. The exact composition of VOCs present in cigarette smoke, however, varies significantly based on burning conditions such as the amount of $\mathrm{O}_{2}$ present and the temperature at the cigarette tip [91]. Notable VOCs include benzene and aldehydes such as acetaldehyde, acrolein, and formaldehyde [92]. Benzene, in particular, is a potent carcinogen contributing $9.5 \%$ to the cancer risk index of cigarette smoke, with aldehydes and other VOCs 
contributing 43.6\% [93]. VOCs [94], including formaldehyde [95] and benzene [96], have been associated with low birth weight in children. Although we did not note any published studies on the development of metabolic syndrome or obesity following prenatal exposure to VOCs, studies should be done to examine this because of how often low birth weight has been associated with obesity in adulthood.

Heavy metals are present in tobacco because of absorption from the soil $[97,98]$. Cadmium [99], lead [99], and mercury [100] are readily absorbed by tobacco plants, which is correlated with the concentration found in the soil. Both cadmium and lead are easily transferred from tobacco into smoke $[101,102]$. In cigarette smoke, cadmium is mostly in the particulate phase, while lead is split into both gas and particulate phases in equal proportions [103]. In addition to being carcinogenic, cadmium has been proposed as an agent contributing to in utero cigarette smoke exposure-induced low birth weight $[104,105]$. It has been hypothesized that cadmium causes this by interfering with placental zinc and copper transfer [104] and inhibiting placental progesterone production [105]. Five-year-old children exposed in utero to cadmium were recently shown to be associated with approximately 25 -fold increased odds of being obese [106]. Prenatal lead exposure has been shown to result in delayed infant growth during the first two years of life, especially if their mothers consumed less than average amounts of calcium [107]. A recent study examining maternal red blood cell lead levels revealed 1.65 times increased odds of having an overweight or obese child by eight years of age on average [108]. It is evident that heavy metals, as well as other components of cigarette smoke discussed in this section, have great potential to cause manifestations of the Barker Hypothesis. This potential makes it all the more prudent to examine the effects of in utero cigarette smoke exposure on the development of adulthood metabolic disease.

\section{Barker Hypothesis}

The Barker Hypothesis, more broadly known as the Developmental Origins of Health and Disease, states that adverse in utero environmental factors result in an increased risk of adultonset diseases [109]. Specifically, it appears that it is low birth weight and not preterm birth that 
results in this increased risk later in life [110]. The amount of nutrition received by the developing fetus has a direct impact on birth weight. It has been shown that children affected by maternal caloric restriction during pregnancy exhibited an increased risk of obesity [111-114], heart disease [112, 115-118], diabetes [115, 119-122], and hypertension [115, 123-125]. Further studies in animals [126-134] and human epidemiological studies [135-137] support the Barker Hypothesis by illustrating instances of maternal caloric insufficiency leading to adulthood manifestations of metabolic disease.

Children exposed to active maternal smoking during pregnancy [138-142] or environmental tobacco smoke after birth [143-147] have been found to have an increased risk of obesity and metabolic syndrome. A recent meta-analysis in 2018 utilizing 16 studies found the odds of children (the mean age of the children ranged from 3.8 to 15 years old) being overweight were increased when mothers smoked 1-15 cigarettes each day [148]. A proposed mechanism for this increased risk of metabolic syndrome development in children is from the Barker Hypothesis-derived concept of the "thrifty phenotype" [149]. This hypothesis predicts in the context of placental insufficiency that the fetus responds to maternal smoking in a manner that is analogous to caloric restriction [150]. Nicotine, acting as a vasoconstrictor, limits blood flow from the mother to the placenta and results in caloric restriction of the developing fetus [150]. It is predicted that as a result, the fetus will develop metabolic adaptations to survive nutrient scarcity. A term that has been coined to describe this phenomenon is predictive adaptive response (PAR) [151]. In other words, the fetus detects through the mother facets of the in utero environment it expects to be born into and undergoes adaptations such as altered insulin activity to promote increased success or survival after birth [151]. Increased peripheral insulin resistance in conjunction with lowered insulin secretion have been posited as adaptations of the thrifty phenotype [152]. Subsequent exposure to a postnatal environment of calorie-dense food induces catch-up growth in these low birth weight offspring [150]. This process of catch-up growth increases the risk of developing metabolic syndrome in response to a high-fat Western diet in adulthood [150]. Further, this hypothesis predicts that male offspring will be more adversely affected by prenatal CSE since they are typically larger and more vulnerable to starvation during fetal development [150]. Considering the significant 
ongoing exposure to in utero cigarette smoke, it is prudent to examine the potential impact on additional metabolic syndrome and obesity burden.

\section{Metabolic Syndrome and Obesity}

Metabolic syndrome, at its simplest, is a condition characterized by obesity, dyslipidemia, and insulin resistance/hyperglycemia [153]. This syndrome is hypothesized to occur because each of these symptoms share underlying pathology and collectively contribute to the risk of developing cardiovascular disease and type 2 diabetes [153]. Over the years, metabolic syndrome has been defined in several ways. The World Health Organization in 1998 required that insulin resistance as measured by fasting glucose or another appropriate method be accompanied by at least two of the following: increased waist/hip ratio or BMI, increased triglycerides or HDL-C, hypertension, or microalbuminuria [154]. A more recent definition from the 2005 revision of the definition established by the National Cholesterol Education Program (NCEP) Adult Treatment Panel III (ATP III) requires 3 out of the following criteria: increased waist circumference, elevated fasting blood glucose, elevated triglyceride levels, lowered HDL cholesterol levels, or elevated systolic or diastolic blood pressure [155]. The International Diabetes Foundation (IDF) requires central obesity to be present, with two of the following: increased fasting blood glucose, triglyceride levels, systolic or diastolic blood pressure, or decreased HDL cholesterol levels [156]. It should be noted that there are multiple additional definitions in current clinical use in addition to the ones mentioned here [153].

According to a recent report from the Centers for Disease Control and Prevention (CDC) that analyzed data between 1992-2012 in the United States from the National Health and Nutrition Examination Survey (NHANES), the prevalence of metabolic syndrome in adults 18 years or older as defined by the NCEP ATP III criteria increased from $25.3 \%$ to $34.2 \%$ [157]. This same CDC report also found that age had a positive association with the prevalence of metabolic syndrome while education level was negatively associated with metabolic syndrome prevalence [157]. At least $50 \%$ of men and $60 \%$ of women by 70 years old had metabolic syndrome as reported in the 2007-2012 NHANES population [157]. Given that the rate of obesity in adults in the U.S. between $2000-2016$ has risen from $30.5 \%$ to $39.6 \%$, as well as the rate of obesity in youth going from $13.9 \%$ 
to $18.5 \%$ within the same time period [158], it seems likely that the rate of metabolic syndrome will continue rising unless drastic measures are taken.

The rates of both obesity and metabolic syndrome have been associated with offspring exposed in utero to cigarette smoke. One study found that 5 year old children born to mothers that smoked during pregnancy were three times more likely to be overweight as defined by BMI [159]. A meta-analysis performed in 2010 utilizing 17 studies found a pooled odds ratio of 1.52 for children becoming obese as a result of exposure in utero to cigarette smoke [160]. Another meta-analysis conducted in 2008 utilizing 14 studies found that children exposed to prenatal cigarette smoke have an increased odds ratio (1.40) for being overweight [138]. Adult women that were exposed in utero to cigarette smoke exhibited elevated triglycerides and lowered HDL cholesterol (two criteria from the NCEP ATP III metabolic syndrome definition) [161]. Both BMI and the prevalence of being overweight or obese were higher in 14-year-old children of mothers who smoked during pregnancy in a 2006 Australian cohort study after adjustment for several factors such as the reported diet of the child [162]. A United Kingdom-based cross-sectional survey also found that mothers who smoked ten or more cigarettes per day during pregnancy had children that exhibited higher BMI and were more likely to be obese at 5-11 years old [163]. Another study found that children that were on average 9.9 years old and exposed to cigarette smoke at any point in utero exhibited higher BMI and total fat mass [164]. Mothers who smoked early in pregnancy went on to have children that were at greater risk of being overweight, and exhibiting a higher BMI and systolic blood pressure at three years old with no evident confounding by child diet [140]. A cohort of 17-19 year old Swedish men followed between 1983-1988 whose mothers smoked during pregnancy were found to have significantly higher systolic and diastolic blood pressure during adolescence [165]. A cohort of two-month old Netherlander infants whose mothers smoked during pregnancy was found to have a significantly increased systolic blood pressure after adjusting for confounders such as childhood nutrition [166]. Ten year old children exposed to prenatal CSE had higher insulin levels and insulin resistance, even more so if their mothers exclusively breastfed [167]. It is evident that cigarette smoke exposure in utero is contributing to obesity and metabolic disease in adulthood, but what is the underlying mechanism driving this pathogenesis? 


\section{Liver Development and Function}

We established in the prior section the import of obesity and metabolic syndrome concerning in utero exposure to cigarette smoke. The question that has yet to be satisfactorily answered is what occurs in the liver of prenatal cigarette smoke-exposed children that results in this increased risk of adulthood metabolic disease? To better understand the potential impacts that prenatal toxicants have on the liver, it is necessary to review facets of hepatic development and function. Liver organogenesis occurs around gestational day 8.5-9 in mice and 23-26 days into human pregnancy, when the liver diverticulum forms from the anterior foregut endoderm that is adjacent to the cardiac mesoderm [168]. It is within this liver diverticulum in which the septum transversum and hepatoblasts begin to appear [168]. These hepatoblasts begin migration into the septum transversum and proliferate while expressing alpha-fetoprotein and albumin [169172], production of the latter being one of the critical functions of the liver. Around the same time that the liver diverticulum is forming, a ring of endothelial cells encircling the diverticulum help to promote liver bud formation and migration of hepatoblasts into the septum transversum at gestation day 9.5 in mice and 26-32 days in humans [168]. Once the liver bud starts forming, hematopoietic progenitor cells begin migrating into the liver bud to establish fetal hematopoietic

function around ten days of gestation in mice and week five in humans $[173,174]$. Hepatoblasts are bipotential progenitor cells that can either differentiate into hepatocytes or cholangiocytes, which occurs around gestational day 13.5 in mice and 56-57 days in humans [168]. Together, these two cell types make up the majority of the cell mass of the liver; cell fate depends on proximity to the portal vein [168]. Hepatoblasts adjacent to the portal vein will differentiate into cholangiocytes, while hepatocytes located away from the portal vein will form hepatocytes [175]. Cholangiocytes eventually start budding off from the ductal plate surrounding the portal vein and then form bile ducts around gestational day 17.5 in mice and 14 weeks in humans where the remaining cholangiocytes in the ductal plate regress $[168,176]$. This process finally ends at about 30 weeks of gestation in the human fetus and within the first ten days after birth in mice $[168,177]$. This process sets up the production of bile, a critical function of the liver during 
development and after birth. During development, the fetal liver in both mice and humans acts as the source of hematopoietic cells between gestational days 9.5-15 in mice and days 60-195 in humans [178]. After this period, hematopoiesis is shifted to the bone morrow while it is decreased in the liver [178]. It is apparent how prenatal toxicants could have lasting consequences on the liver if they occur at key hepatic developmental stages.

The final phase of maturation for hepatocytes occurs within hepatic lobule zones, which specify the location of hepatocytes relative to the portal and central veins. This process begins after the differentiation of hepatoblasts into hepatocytes and continues after birth [178]. In particular, the expression of many phase I and phase II metabolic enzymes are low in fetal livers but increase after birth [178]. The hepatocyte population becomes heterogeneous in nature with respect to what lobular zone they occupy [179]. Those closest to the portal vein in which higher oxygen concentrations are found become more focused on oxidative processes such as fatty acid oxidation and glutathione production [179]. Lobular zones surrounding the central vein and thereby have a lower concentration of oxygen becomes focused more on non-oxidative processes like glycolysis and lipogenesis [179]. Other primary functions of the liver that have not been mentioned, which are described in further detail in subsequent paragraphs, are synthesizing and storing glycogen as well as urea synthesis from nitrogen-containing compounds such as ammonia. It is evident how prenatal toxicants could impact hepatic function by disrupting the organization of hepatoblasts into metabolic functional units.

The mature liver is vital for glucose metabolism and maintaining blood glucose levels. After the consumption of a meal, glucose is taken up in hepatocytes after being brought into the liver via the portal vein from the small intestine [180]. After being brought into hepatocytes, glucose is is phosphorylated into glucose-6-phosphate by glucokinase, which effectively prevents glucose from diffusing out of hepatocytes [180]. It is at this point that glucose can meet several different fates depending on physiological needs. One pathway glucose enters after a meal is glycogenesis in which glycogen is stored in the liver via the conversion of glucose-6-phosphate into glucose-1-phosphate [181] followed by production of UDP-glucose [182]. Glycogen is formed from the conversion of UDP-glucose by glycogen synthase [183]. During development, the drive 
for glycogen storage is induced by expression of a large amount of Hexokinase I, which ensures glycogen is produced and stored regardless of circulating glucose levels [184]. Glycogen can also be converted back into glucose-6-phosphate and glucose can then be secreted into the circulatory system to maintain blood glucose levels [183]. Glucose-6-phosphate can also be used as a substrate for glycolytic and tricarboxylic acid pathways to produce ATP to power various cellular functions [183] or utilized in fatty acid synthesis [185]. Additionally, glucose-6-phosphate can be used to produce UDP-glucuronate [186] and UDP-galactose [183] which serve as monosaccharide donors for the glycosylation of proteins to induce differential activity [183]. The last significant fate of glucose-6-phosphate is the pentose phosphate pathway, in which NADPH is produced as a cofactor for many synthetic reactions [187]. The NADPH produced by the pentose phosphate pathway is also utilized in the synthesis of the ribose-5-phosphate necessary to produce nucleic acids [187]. The liver also produces glucose, particularly during fasting, and can do so via two pathways. The first of these pathways which has already been mentioned is glycogenolysis, in which glycogen is broken down into glucose-1-phosphate then converted back to glucose-6-phosphate [188]. After these steps, glucose-6-phosphate is dephosphorylated into glucose [189] that can leave the liver and go into circulation [188]. The other pathway produces glucose via gluconeogenesis, which can take several substrates like lactate or alanine and convert them to pyruvate [183]. This pyruvate can then be combined with acetyl-CoA to form oxaloacetate [190], then converted to phosphoenolpyruvate [191], then enter the glycolytic pathway in reverse until it reaches fructose-1,6-bisphosphate [183]. The next step is irreversible and converts fructose-1,6-bisphosphate into fructose-6-phosphate [192]. After this step, glucose6-phosphate is formed and can be dephosphorylated to glucose [189] and can then be shunted back into circulation [183]. During the initial stages of fasting, glycogenolysis is the principal source of glucose production, but as glycogen stores are depleted, gluconeogenesis becomes predominant and by 48 hours is the primary source of glucose synthesis [193]. It is evident how prenatal toxicants that affect the liver have the potential to perturb normal glucose metabolic activity and increase the risk of adulthood metabolic disease. 
Lipid metabolism is a crucial function of the liver, as it allows for the long-term storage of energy obtained from food [194]. Lipid metabolism also provides substrates used for the synthesis of specialized lipids for plasma membranes and bile acid production [194]. After dietary fat has been digested, the resultant fatty acids are converted into triacylglycerols (TAG) [194]. TAGs are stored in hepatocytes as lipid droplets or they are shuttled out into general circulation as very-low-density lipoprotein (VLDL) [194]. De novo lipogenesis is another essential source of fatty acids that are derived from the hepatic conversion of glucose into fatty acids after feeding. Glucose is initially metabolized through the glycolytic pathway until it becomes pyruvate after which it is converted into acetyl-CoA in the mitochondria [194]. Acetyl-CoA combines with oxaloacetate to form citrate which is then converted in the cytoplasm back into acetyl-CoA and oxaloacetate [194]. Once out in the cytoplasm, acetyl-CoA is converted into malonyl-CoA [195], which serves as a 2-carbon substrate for the NADPH-powered synthesis of fatty acids [196], specifically palmitic acid [194]. Note that this process of lipogenesis only occurs after feeding when excess glucose and glycogen stores have been replenished [197]. During a fasted state, a process called lipolysis linked with $\beta$-oxidation is utilized to produce ATP as well as substrate for gluconeogenesis [194]. TAGs are initially broken down into glycerol and fatty acids, in which the glycerol unit is able to be used as a gluconeogenic input [194]. The fatty acids are then broken down within mitochondria via $\beta$-oxidation, where two-carbon atom units are repeatedly removed from the terminal end of the fatty acid until it is completely broken down [198]. Each round of $\beta$ oxidation produces ATP as well as an acetyl-CoA molecule, which can be used as an input into the TCA cycle to produce even more ATP [198]. It is evident how prenatal toxicants that affect the liver can increase the risk of adulthood metabolic disease by their potential to disrupt lipid metabolism.

Detoxification of both endogenous and exogenous compounds is an important task performed by the liver and is carried out in two primary phases. The first of these phases is referred to as phase I reactions, which serve to add a reactive site to compounds through processes such as hydroxylation [199], though other reaction types are used as well, such as carboxylation [200]. The purpose of this phase is to render chemically inert compounds to be 
susceptible to conjugation by other enzymes [200]. The family of enzymes responsible for carrying out phase I reactions are collectively known as the cytochrome P450 enzymes. These enzymes are comprised of heme-thiolate proteins, of which there are numerous members. The naming convention for these enzymes typically follow the letters CYP followed by a number for their family, which were historically Roman numerals [201]. Following these first parts is the subfamily letter and then the subfamily member number as each member is reported to the P450 Nomenclature Committee (CYP1A1 would be the very first P450 enzyme, for example) [201]. It is hypothesized that the evolution of this family of enzymes came about mainly for the processing of endogenous compounds [200]. The fact that these enzymes are useful for processing exogenous and xenobiotic compounds is due mainly to structural similarity to endogenous compounds [200]. It is also hypothesized that P450 enzyme diversity was also driven in part by the employment of toxic compound synthesis in plants to counteract herbivory, resulting in herbivore adaptation by employing an expanding repertoire of P450 enzymes to neutralize toxic plant compounds $[202,203]$. Given the sheer number of compounds that are found in cigarette smoke, it is evident how prenatal exposure to these compounds during development when liver phase I enzyme function has not fully formed can result in hepatic toxicity.

The second set of major detoxification enzymes carry out phase II reactions in which the compounds initially processed by phase I enzymes are then conjugated with hydrophilic molecules [204]. Generally, these conjugation reactions render compounds more water-soluble so they can be excreted through the bile or the excretory system [204]. One of the most significant phase II enzyme families is the UDP-glucuronosyltransferases, which catalyze the conjugation of compounds with glucuronic acid using UDP glucuronic acid [205, 206]. This family of enzymes is estimated to be responsible for approximately $40-75 \%$ of xenobiotic detoxification $[205,206]$. Sulfotransferases (SULTs) are another critical group of phase II enzymes that conjugate a hydrophilic group onto compounds, specifically a sulfuryl group [207, 208]. This reaction is applied to either hydroxyl or amine residues and generally render a compound more hydrophilic as well as less toxic or biologically active [207]. Glutathione-S-Transferases (GSTs) conjugate compounds with a molecule of glutathione in response to electrophiles or electrophile- 
generating compounds and reactive oxygen species (ROS) [209]. It is hypothesized that GST induction occurs as an adaptive response ROS and thereby results in GST reactions with hydroxyl, carbonyl, peroxide, and epoxide residues [210, 211]. Another major family of phase II enzymes are $\mathrm{N}$-acetyl transferases (NATs) that conjugate aromatic amine- or hydrazinecontaining compounds with acetyl groups [212]. Polymorphisms in NAT-related genes can manifest different acetylator phenotypes in which slow acetylators have been associated with an increased risk of developing liver and colon cancer [213]. NAT-related gene polymorphisms have even been associated with cleft palate risk in infants with mothers that did not take prenatal multivitamins [214]. The last major group of phase II detoxification enzymes is the methyltransferases, which conjugate a methyl group onto compounds by using methionine from S-adenosylmethionine (SAMe) [212]. The two types of methyltransferases are thiopurine methyltransferase (TPMT) and catechol-O-methyltransferase (COMT) [212]. COMTs are essential not just in the inactivation of catecholamines such as dopamine, epinephrine, and norepinephrine [212, 215], but also inactivation of catechol-estrogens and metabolites [216]. TPMT prototypically metabolizes thiopurine drugs such as azathioprine [217]. Polymorphisms affecting TPMT activity are essential in determining the toxicity of thiopurine drugs like 6mercaptopurine [218]. It is evident how prenatal exposure to cigarette smoke compounds during a time in which phase II enzymatic activity may not be fully developed can result in fetal hepatic toxicity.

Throughout development and after birth, there are multiple trajectory types with respect to drug-metabolizing enzyme expression: high expression during gestation with decreased expression after birth (Class 1), constant expression pre- and postnatally (Class 2), and low expression during gestation followed by increased expression after birth (Class 3) [219]. Examples of Class 1 enzymes include CYP3A7 [220, 221] and SULT1E1 [222] which have been hypothesized to follow a Class 1 pattern because they serve a necessary function during gestation that is no longer needed after birth [219]. For example, SULT1E1 has been hypothesized to inactivate estrogens in order to promote androgenic activity at critical male gonadal developmental windows [223]. Class 2 enzyme examples include CYP2B6 [224] and 
SULT1A1 [222], and while there is not a particular hypothesis about why these enzymes are expressed continuously during both gestation and after birth, it is thought that they serve different roles before and after birth or they carry out the same function during both life stages [219]. Examples of Class 3 enzymes include CYP3A4 [220, 221] and SULT2A1 [225] and are predicted to express high levels after birth but not during gestation because of more contact with various environmental hazards and thereby need to be able to handle these exposures when encountered [219]. It is evident how prenatal exposure to cigarette smoke exposure can be variable depending on the enzymes responsible for metabolizing a particular cigarette smoke compound and when those enzymes are normally expressed.

Bile acids are amphipathic compounds synthesized in the liver from cholesterol, where the rate-limiting enzyme is CYP7A1 [226]. Bile acids emulsify fats, cholesterol, and fat-soluble vitamins in the small intestine to aid in the maximal absorption by enterocytes [227]. Typically, $\sim 95 \%$ of bile acids are reabsorbed in the small intestine after which they are transported to the liver via the portal vein for reabsorption and subsequent resecretion into enterohepatic circulation [227]. The remaining $5 \%$ is excreted and replaced by compensatory hepatic synthesis [227]. Primary bile acids, such as chenodeoxycholic (CDCA) and cholic acid (CA), are produced in the liver [227]. These primary bile acids are then conjugated with either taurine or glycine to be stored in the gallbladder for postprandial secretion into the duodenum of the small intestine [227]. Conjugation of bile acids occurs is carried out by enzymes such as BACS [228] and BAT [229], as well as other conjugation enzymes typically utilized for detoxification, including SULT2A1 [230] and UGT2B4 [231]. Conjugation by these enzymes render primary bile acids more hydrophilic and amphipathic [232]. In the rat small intestine, taurine-conjugated bile acids are the majority in both the duodenum and ileum, whereas the large intestine is enriched in unconjugated bile acids [233]. Gut microbiota deconjugate bile acids via bile salt hydrolase and result in the release of glycine or taurine; conversion of primary bile acids into more hydrophobic secondary bile acids such as deoxycholic acid (DCA) occurs via 7a-dehydroxylase [234-236]. It is evident that prenatal exposure to toxicants that can disrupt the development of the liver could result in 
perturbed bile acid metabolism in offspring, thereby potentially resulting in an altered bile acid pool composition with physiological consequences.

Sexual differentiation is an important developmental facet that helps determine the functional hepatic phenotype. Back in the 1930s it was noted that male rats given the same dose per kilogram of bodyweight of hexobarbital slept for a shorter duration than female rats and that this difference was abolished when male rats were castrated $[237,238]$. Since then, it has been found that one of the significant determinants for these sex differences in hepatic function is the patterning of growth hormone (GH) secretion from the anterior pituitary gland. By using an osmotic pump to mimic female-specific constant GH secretion in male mice in which the secretion of GH has been eliminated by the neonatal administration of monosodium glutamate, the hepatic expression of CYP2A4, CYP2B9, CYP2B10, CYP3A41 was increased while CYP2D9 was decreased [239]. Conversely, female mice with GH secretion eliminated via neonatal monosodium glutamate administration that were given twice daily $\mathrm{GH}$ injections to mimic the male

GH secretion profile resulted in suppressed hepatic expression of CYP2A4, CYP2B9, CYP2B10, and CYP3A4 while promoting expression of CYP2D9 [239]. Other recent studies have also demonstrated this system of sex-specific GH secretion-induced hepatic gene expression for hepatic gene methylation [240], regulation of miRNA expression [241], and direct and indirect mediation by hepatic STAT5b and HNF4a [242-244]. Given the sex-specific effects on hepatic gene expression and function, an examination of the sex-specific hepatic impacts of prenatal cigarette smoke exposure should be performed to fully characterize the risk of metabolic disorders in offspring.

\section{Non-Alcoholic Fatty Liver Disease}

Non-alcoholic fatty liver disease (NAFLD), which at its simplest is the presence of hepatic steatosis (or fat accumulation in the liver), requires two criteria in order to be diagnosed [245]. The first criterion is confirmed evidence of hepatic steatosis either by imaging such as through ultrasound or by liver biopsy, which is considered to be the gold standard for identifying hepatic steatosis [245]. The second criterion is that nothing else is present which could cause hepatic fat 
accumulation such as significant alcohol consumption, a genetic disorder, or the use of medication that is known to cause steatosis such as corticosteroids, tamoxifen, amiodarone, and others [245]. NAFLD commonly presents with mild to moderate steatosis and hepatocellular ballooning [246]. When non-alcoholic steatohepatitis (NASH) is present in NAFLD patients, it presents with lobular inflammatory that manifests as immune cell infiltration by mononuclear and polymorphonuclear cells [246]. When the condition of NASH has progressed into the fibrotic stage, it often presents in a pericellular manner [246]. Given the potential severity of NAFLD and $\mathrm{NASH}$, it is essential to investigate the possibility of prenatal cigarette smoke exposure to increase the risk of developing these conditions during adulthood.

NAFLD is increasingly becoming a global problem and is currently the most significant cause of chronic liver disease in the world. The global prevalence of NAFLD was estimated to be $25.24 \%$ by a meta-analysis published in 2016 utilizing 86 studies [247]. This same meta-analysis found the prevalence of NALD was lowest in Africa (13.48\%), followed by Europe (23.71\%), North America (24.13\%), Asia (27.37\%), South Africa (30.45\%), and the Middle East (31.79\%) [247]. This meta-analysis also made clear that NAFLD was often accompanied by related metabolic disorders such as obesity (51.34\%), hyperlipidemia (69.16\%), hypertension (39.34\%), Type 2 diabetes (22.51\%), and metabolic syndrome (42.54\%) [247]. These comorbidities were even more prevalent when NASH was present, where obesity occurred with NASH at $81.83 \%$, hyperlipidemia at $72.13 \%$, hypertension at $67.97 \%$, type 2 diabetes at $43.63 \%$, and metabolic syndrome at $70.65 \%$ [247]. NASH was suspected of having an overall prevalence of somewhere between $1.5 \%$ and $6.45 \%$, but had a prevalence of $59.1 \%$ in biopsy-confirmed NAFLD patients [247]. Progression of fibrosis in NASH patients was found to occur at an average annual rate of $0.09 \%$ [247]. The annual incidence of HCC in NAFLD patients was found to be 0.44 per 1,000 person-years and 5.29 per 1,000 person-years for NASH patients [247]. The severity of NASH relative to NAFLD can be seen explicitly with the incidence rate ratio (IRR) for liver-specific mortality and overall mortality. NAFLD and NASH patients had an IRR for liver-specific mortality of 1.94 and 64.6, respectively, while NAFLD and NASH patients had an IRR for overall mortality of 1.05 and 2.56 , respectively [247]. This data only serves to confirm precisely why the 
increasing rates of NAFLD and, subsequently, NASH are so disconcerting. The evidence clearly underlines the health burden on morbidity and mortality these diseases and their comorbidities will have on the global population. These data are especially concerning due to NASH-related cirrhosis being the second leading cause for liver transplants in the U.S. between 2004-2013 [248]. This same study found that the 90-day survival rate for NASH patients on the transplant waitlist was lower than patients with alcoholic liver disease, hepatitis $C$ infection, or patients with both alcoholic liver disease and hepatitis C [248]. This lower survival rate in NASH patients was due to their lower likelihood to receive a liver transplant at 90 days compared to other patients [248]. NASH patients that were added to the waitlist for liver transplantation went up by $170 \%$ between 2004-2013, whereas it increased by only $45 \%$ for alcoholic liver disease patients and $14 \%$ for hepatitis C patients [248]. If the current trend of ever increasing NAFLD and NASH prevalence continues unabated, the already strained supply of liver transplants will become a fullblown crisis.

Cigarette smoke exposure also has been known to impact the development and severity of NAFLD. A retrospective study looking at the development of NAFLD in patients over a 10-year period demonstrated that smoking (1.91 adjusted OR) in addition to being male (1.46 adjusted OR), having a BMI 25 or higher (3.08 adjusted OR), and dyslipidemia (1.79 adjusted OR) all increased the odds of NAFLD diagnosis [249]. Additionally, the odds of developing NAFLD were higher in patients with a Brinkman Index, defined by the product of cigarettes smoked daily and the duration of smoking in years, between 1-399 (1.77 times higher) or 400 and higher (2.04 times higher), which clearly demonstrated a dose-dependent effect [249]. A study utilizing the NASH Clinical Research Network found that NAFLD patients with a history of ten or more years of smoking was associated with odds 1.63 times higher for advanced fibrosis [250]. This study also found that these same NAFLD patients had increased odds for each year of age (1.06 times higher), or were diagnosed with diabetes (2.44 times higher) [250]. There has even been evidence that genetic polymorphisms for Adiponectin and GPx-1 that were positively associated with cigarette smoking-induced NAFLD risk [251]. If even some of the effects of cigarette smoke 
exposure on the development of NAFLD are also present with in utero cigarette smoke exposure, it is all the more essential to study the impacts of prenatal CSE on adulthood metabolic disease.

Pediatric cases of NAFLD differ from the typical presentation of this disease in a couple of ways. In particular, the steatosis tends to be more severe with a lower frequency of hepatocytic ballooning, and any associated inflammation is found more in the portal regions while fibrosis is present more in the periportal regions [246]. This is in contrast to adult NAFLD cases in which any associated inflammation is more lobular in location while fibrosis is detected more often within pericellular regions [246]. The number of pediatric NAFLD cases that resemble an adult presentation were small, but the majority of pediatric cases presented themselves as previously described [246]. The remainder of the pediatric NAFLD cases that do not manifest themselves in the previously mentioned ways had some mixture that was between the typical adult and pediatric NAFLD presentation [246]. A meta-analysis found that the prevalence of pediatric NAFLD overall was $7.6 \%$ in the general population [252]. However, when considering studies using a clinically obese population, the prevalence was $34.2 \%$, which was over four times higher than the general prevalence [252]. The odds of children having NAFLD were higher in males than females in a general population (1.63 times higher) and in clinical populations (2.02 times higher) [252]. The prevalence of NAFLD in clinical pediatric populations was found to be higher in Asia (62.3\%) compared to North America (39.2\%), the Middle East (36.5\%), Europe (29.8\%), and South America (17.1\%) [252]. RNAseq analysis of human fetal livers from 12-19 weeks of gestation found that a number of NAFLD-related pathways were upregulated, such as steatosis and liver inflammation, in response to prenatal cigarette exposure [253]. As far as we know, it has not been explicitly studied if prenatal cigarette smoke exposure results in increased rates of pediatric NAFLD cases. However, we may be able to infer the risk of pediatric NAFLD from in utero cigarette smoke exposure from examining the effect of intrauterine growth restriction on pediatric NAFLD risk, which the literature suggests is induced via increased insulin resistance [254]. It is evident that prenatal cigarette smoke exposure may have an impact on the development of not only adulthood NAFLD risk, but potentially childhood NAFLD risk as well. It is 
essential to examine effects of in utero cigarette smoke exposure on metabolic disease development in adulthood, but also at other life stages.

\section{Hepatic Metabolic Regulation by SIRT1}

SIRT1 is NAD+-dependent class III deacetylase [255]. SIRT1 activity induces deacetylation of various transcription factors as a result of sensing low energy status through levels of $\mathrm{NAD}^{+} / \mathrm{NADH}$ [255]. This deacetylation action, as a part of the fasting response, results in the induction of gluconeogenesis, fatty acid oxidation, as well as the suppression of lipogenesis [255]. Feeding results in a high energy state, in which there are lower levels of $\mathrm{NAD}^{+}$, thereby lowering the activity of SIRT1 [255]. Consumption of a high-fat diet, which also results in a high energy state, lowers SIRT1 activity as well via decreased levels of $\mathrm{NAD}^{+}$[255]. In general, the deacetylation of proteins by SIRT1 is opposed by p300-induced acetylation [256]. SIRT1-induced deacetylation of FXR enhances FXR's transactivation activity on its downstream targets by increasing the frequency in which FXR interacts with its partner heterodimer Retinoid X Receptor alpha (RXRa) [256]. In addition to SIRT1's metabolic actions, NF-kB deacetylation by SIRT1 results in lowering NF-kB's transactivation activity on the expression of various pro-inflammatory mediators, thereby affecting immune function [257]. Given how prenatal CSE is hypothesized to induce caloric restriction by limiting blood flow to the developing fetus, it would be highly pertinent to investigate any impact of in utero cigarette smoke exposure on SIRT1 activity.

SIRT1-induced deacetylation also affects protein stability. CRTC2, an essential component in the promotion of gluconeogenesis, becomes more susceptible to ubiquitin-mediated proteasomal degradation once it has been deacetylated [258]. Deacetylation by SIRT1 can promote protein stabilization, a prime example of which is FOXO protein deacetylation, which inhibits their phosphorylation and their ubiquitination-mediated proteasomal degradation [259, 260]. The final mechanism in which deacetylation by SIRT1 affects proteins is by histone deacetylation. Histone deacetylation causes the adjacent DNA to be more tightly associated with its histones, thereby limiting the accessibility of this DNA for transcription. SIRT1 downregulates the expression of miR-34a via this mechanism of histone deacetylation [261]. This action of SIRT1 is particularly 
of note since miR-34a inhibits SIRT1 activity via the inhibition of NAD+ production by NAMPT [262]. miR-34a also directly inhibits transcription of SIRT1 by binding to the 3'-UTR of Sirt1 mRNA [261, 263], thereby affecting both the production and activity of SIRT1. It is evident that characterizing the impact of prenatal CSE on the deacetylation of protiens would be an effective way of investigating how in utero cigarette smoke exposure may be influencing SIRT1 activity in affected offspring.

SIRT1 activity and subsequent downstream pathway activation can be altered by several modulators. As will be discussed later, there is a regulatory positive feedback loop shared with FXR that has a considerable impact on SIRT1 deacetylation activity and system-wide protein acetylation status [256, 261, 264, 265]. The main factor in terms of regulation of SIRT1 activity is the availability of $\mathrm{NAD}^{+}$, which is a necessary cofactor for SIRT1-catalyzed deacetylation [266]. This factor is essential to consider when examining SIRT1 activity since a lack of $\mathrm{NAD}^{+}$can result in a failure of deacetylation to be carried out by SIRT1 even in the presence of excess SIRT1 protein levels [267]. This mechanism is how miR-34a negatively regulates SIRT1 via inhibition of NAMPT, an NAD+-producing enzyme [262]. Likewise, AMPK positively upregulates SIRT1 deacetylation activity by promoting the transcription of NAMPT [268]. Other factors that affect the availability of $\mathrm{NAD}^{+}$can also influence SIRT1 activity. This is the case with Poly ADP-Ribose Polymerase 1 (PARP-1) [269] and CD38 [270] consumption of $\mathrm{NAD}^{+}$pools, thereby indirectly inhibiting SIRT1 activity. SIRT1 is modulated by various protein interactions, such as the binding of AROS to the n-terminus of SIRT1 that further activates SIRT1-induced deacetylation [271]. DBC1 that is bound to SIRT1's catalytic domain is capable of directly impairing SIR1's deacetylase activity [272]. SIRT1 activity can also be modulated by various post-translational modifications. SUMOylation of SIRT1, for example, increases the half-life of SIRT1 protein by promoting its stability [273]. Ubiquitination results in SIRT1 translocation into the nucleus [274], which increases the rate of deacetylation of nuclear proteins. SIRT1 can also be phosphorylated, which results in increased SIRT1-induced deacetylation [275]. Although it has been shown that SIRT1 is a crucial regulator of metabolic activity during caloric restriction, these modifications can occur even during a well-fed state, indicating that SIRT1 has regulatory functions outside of metabolism [273-275]. It 
is evident that investigation of the impact of prenatal CSE on SIRT1 deacetylase activity can be characterized in part by any perturbations caused by in utero cigarette smoke exposure found in factors known to modulate SIRT1-induced deacetylation.

SIRT1 is involved in a regulatory feedback loop with FXR and miR-34a. SIRT1 increases the downstream activity of FXR through deacetylation, which increases the expression of the FXR target protein SHP. This increase in SHP protein expression has an inhibitory effect on p53, which results in less expression of miR-34a, a known direct and indirect inhibitor of SIRT1. Inhibition of miR-34a transcription allows higher SIRT1 expression as well as greater SIRT1 deacetylation activity. This regulatory feedback loop comes into play in many metabolic processes, which is mainly exhibited in rodent models of obesity $[256,262]$. One study showed that obesity caused an increase in miR-34a expression [262], which lead to a decrease in activity from both SIRT1 and FXR [256, 262]. This decrease in SIRT1 and FXR activity induced metabolic changes including increased expression of lipogenic genes [256, 262], decreased levels of bile acid export proteins [256], increased levels of serum VLDL and LDL, and decreased serum HDL levels [256]. These SIRT1-related pathways are illustrated in more detail in Figure 1. Examining the SIRT1 regulatory loop with FXR and miR-34a and impacted downstream targets provide an opportunity to characterize impacts that prenatal CSE may have on SIRT1 activity in affected offspring.

There are recent examples in the literature suggesting that hepatic SIRT1 plays a vital role in the development of NAFLD. In one study, it was found that mice fed a high-fat diet for 16 weeks resulted in greater PARP activation and lower levels of $\mathrm{NAD}^{+}[276]$. These changes ultimately contributed to lower levels of SIRT1 activity and higher levels of lipogenic SREBP-1 activity [276]. Mice with disabled SIRT1 catalytic activity that were fed a high-fat diet for 34 weeks exhibited higher levels of SREBP-1 protein expression with decreased phosphorylation of the pro-SIRT1 factors AMPK and LKB1 [277]. Mice given a NAMPT inhibitor and fed a high-fat diet for 12 weeks resulted in lower levels of $\mathrm{NAD}^{+}$, which led to attenuated SIRT1 activity and levels along with decreased deacetylation of the pro-lipogenic transcription factor SREBP-1 [278]. One study found that the expression of miR-181b, which inhibits transcription of Sirt1 as well as its translation into protein, was increased in NAFLD patients in addition to decreased SIRT1 expression [279]. This same 


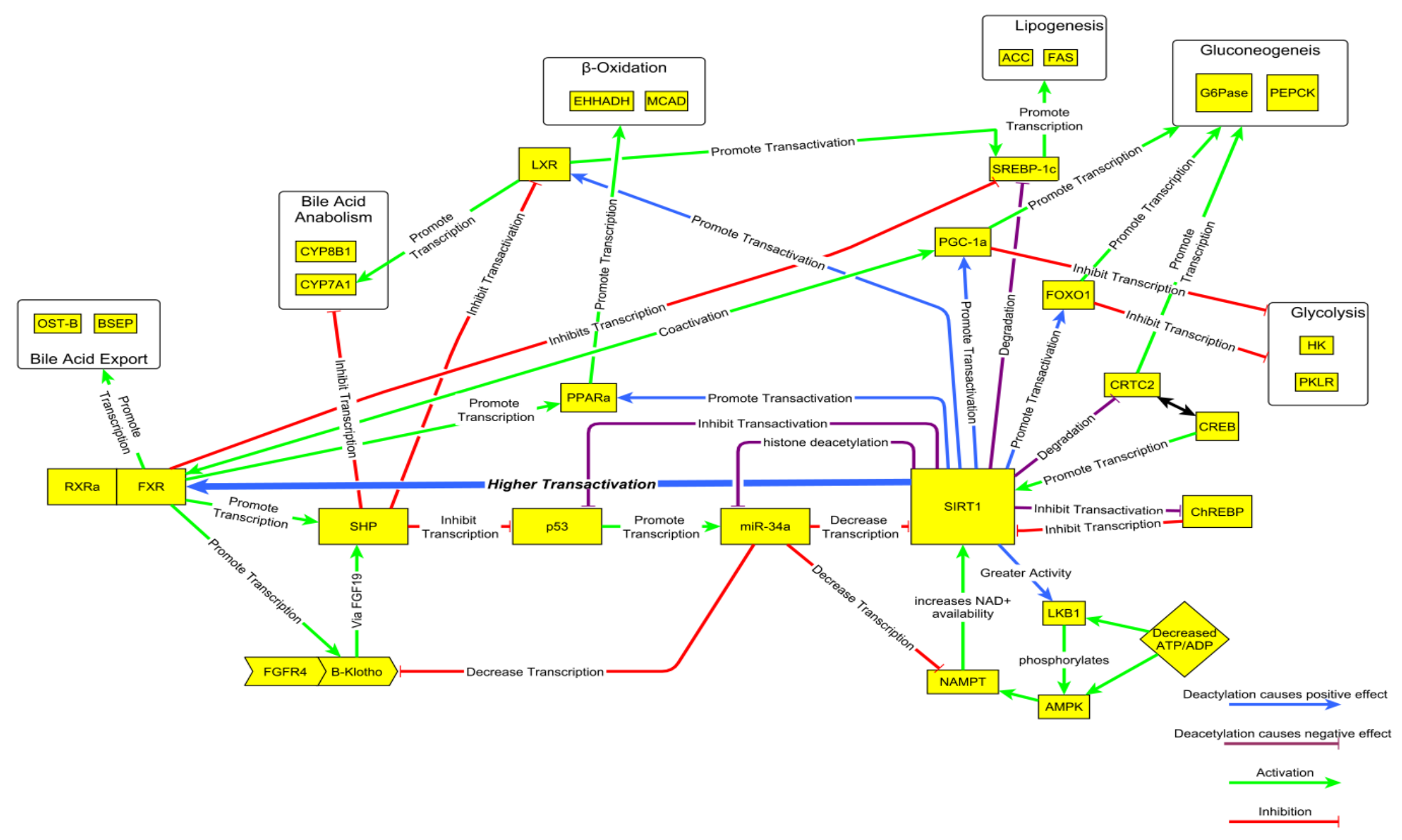

Figure 1 - SIRT1 Regulatory and Downstream Target Pathways 
study also found that mice with high-fat-diet-induced NAFLD exhibited increased miR-181b with a concomitant decrease in SIRT1 expression [279]. In light of this evidence and the hypothesized increase in obesity in adults that have been subjected to prenatal cigarette smoke exposure, it is prudent to examine what role hepatic SIRT1 may play in the manifestation of the Barker Hypothesis and the development of adulthood metabolic disease.

\section{Study Objectives}

The objective of this study was to elucidate the neonatal and adult sex-specific phenotypic characteristics of mouse offspring exposed in utero to cigarette smoke. The study documented herein was designed using a model of murine whole-body inhalation exposure to in utero cigarette smoke via the use of the Teague TE-10C cigarette smoking apparatus. Metabolic characteristics were recorded since this is important in establishing whether an altered metabolic phenotype, as predicted by the Barker Hypothesis, does manifest in our model.

Due to the importance of SIRT1 in regulating the metabolic response of the liver to a lowenergy state, hepatic expression of SIRT1 and the expression of hepatic genes and proteins in related metabolic pathways were examined. These pathways include glucose, lipid, and bile acid metabolic processes known to be modulated by SIRT1 activity. The characterization of these hepatic gene and protein changes during the neonatal period, post-weaning, and during adulthood will allow us to determine if any early changes observed in the liver are associated with perturbations found during adulthood, especially in conjunction with high-fat diet feeding. There are future studies currently ongoing observing adult siblings of the neonatal offspring described in this dissertation. Ultimately, conducting this study will allow insight into how in utero cigarette smoke exposure alters the hepatic metabolic phenotype and fulfills the prediction of the Barker Hypothesis of obesity development during adulthood. The objectives of the study are as follows:

1) Establish whether in utero CSE results in sex-specific diet-induced obesity coupled to impaired SIRT1 expression during adulthood. 
2) Determine the sex-specific impact of prenatal cigarette smoke exposure on SIRT1-related hepatic gene and protein expression at 1.5 weeks, an age at which catch-up growth has occurred, and liver development is still ongoing.

3) Determine the sex-specific impact of prenatal cigarette smoke exposure on SIRT1-related hepatic gene and protein expression at weaning in both the fed and fasted state. 
CHAPTER II: PRENATAL CIGARETTE SMOKE EXPOSURE IMPACTS ADULTHOOD WEIGHT GAIN AND SIRT1-ASSOCIATED METABOLIC PATHWAYS DISTINCTLY BY SEX

\section{Introduction}

In 2016, $7.2 \%$ of U.S. women reported smoking at any point during pregnancy [47]. This rate translates to at least 284,000 fetuses per year exposed to in utero to cigarette smoke [47]. Although many states have reported smoking prevalence in expectant mothers of $5 \%$ or less, several states have rates above $15 \%$ with Kentucky (18.4\%) and West Virginia (25.1\%) being the highest [47]. An analysis of 1999-2006 NHANES data indicated a significant level of underreporting of smoking by pregnant women (22.9\%) after confirming serum cotinine levels [51]. Assuming this figure is still accurate for 2016, this means as many as 22901 out of 55449 infants born in Kentucky in 2016 were exposed to in utero cigarette smoke [47].

A significant primary outcome of cigarette smoke exposure (CSE) during gestation in infants is intrauterine growth restriction (IUGR), which presents as low birth weight $[28,29]$. A Meta-analysis found that in utero cigarette smoke exposure increases the rate of various congenital disabilities, including orofacial clefts, congenital heart defects, gastroschisis, craniosynostosis, clubfoot, anorectal atresia, musculoskeletal defects, hernias, and undescended testes [31]. It has been found that the risk of Sudden Infant Death Syndrome was more than double with children exposed to secondhand smoke in utero through active maternal smoking [32]. Murine models of prenatal CSE exhibit low birth weight [35], decreased crown-rump length [41] as well as decreased somite number [42]. The majority of murine [35-41] as well as rodent [43] models of prenatal CSE exhibit a similar low birth weight phenotype.

The Barker Hypothesis proposes that caloric restriction during pregnancy, resulting in low birth weight, increases the risk of obesity [111], heart disease [115], diabetes [115, 119-122], and 
hypertension [115, 123-125]. Further studies in animals [126-129], as well as human epidemiological studies [32-45], support the Barker Hypothesis by illustrating instances of caloric insufficiency leading to manifestations of metabolic disease later in life. The Barker Hypothesis predicts, in the context of prenatal CSE, that the fetus will respond to maternal smoking in a manner that is analogous to calorie restriction [150].

Nicotine, acting as a vasoconstrictor, limits blood flow from the mother to the placenta and in doing so, results in caloric restriction of the developing fetus [150]. Prenatal exposure to other cigarette smoke constituents have been shown to be associated with low birth weight or fetuses presenting with small for gestational age including carbon monoxide exposure [280, 281], benzo(a)pyrene [89], volatile organic compounds [94] including specifically formaldehyde [95] and benzene [96], polycyclic aromatic hydrocarbons [87, 88], and heavy metals like cadmium [282285]. As a result of the fetus developing metabolic adaptations to survive nutrient scarcity, subsequent exposure to a postnatal environment of calorie-dense food induces catch-up growth in these low birth weight offspring which simultaneously increases the risk of developing metabolic syndrome in response to a high-fat Western diet [150, 286, 287]. Further, this hypothesis predicts that male offspring will be more adversely affected by prenatal CSE since they are more vulnerable to starvation during development [150].

Children exposed to active maternal smoking during pregnancy [138-142], as well as to environmental tobacco smoke after birth [143-147], have been found to have an increased risk of obesity and metabolic syndrome. Defining metabolic syndrome in children is complicated by the fact that the biomarkers used as criteria naturally vary with age [288]. To illustrate how ill-advised applying adult criteria onto pediatric cases is, a cross-sectional study examining metabolic syndrome prevalence in children age 10 to 18 years using multiple definitions found that the prevalence ranged from $0.9-11.4 \%$ depending on the definition used [289]. In light of this, the International Diabetes Federation has attempted to set age-specific criteria: for 10-16 year olds, use IDF criteria set for adults except use the age-specific $90^{\text {th }}$ percentile for waist circumference cut-off instead of a specific circumference; for children 16 years and older, use the normal adult IDF criteria; for 6-10-year-olds, they state it simply cannot be diagnosed in this age range, but it 
should be noted if there is an age-specific waist circumference above $90^{\text {th }}$ percentile [290]. One study that has examined the effects of in utero cigarette smoke exposure on cardiovascular disease in weaned offspring in a murine model found that female CSE offspring fed a high-fat diet for two weeks gained more weight than male CSE offspring and perturbed total cholesterol and highdensity lipoprotein levels [46]. Additionally, our lab has also contributed several murine studies of combined pre- and postnatal exposure to cigarette smoke that induced low birth weight along with altered organ-specific proteomic changes after weaning [35-37] and later in adulthood [38-40] that show alterations in metabolic pathways.

Maternal stress and glucocorticoid levels are potential mediators of the Barker Hypothesis, as well. A report by the CDC found that in $2010,70.2 \%$ of women experience at least on stressful life event in the year before the birth of their child [291]. Maternal depression, another factor thought to influence offspring outcomes, in 2015 occurred at a rate of 28.7 per 1000 delivery hospitalizations, according to a recent study [292]. Although it is not agreed on exactly how the hypothalamus-pituitary-adrenal (HPA) axis is affected in offspring following elevated maternal stress or glucocorticoid levels during pregnancy, there have been numerous studies documenting effects on offspring HPA axis function from these maternal exposures [293]. Of note is a study where the offspring of Wistar rats injected with nicotine twice daily $(1.0 \mathrm{mg} / \mathrm{kg})$ from gestational day 11 until parturition and subjected to chronic stress exhibited exacerbated HPA-axis response in addition to the sensitization of the HPA-axis caused by the chronic maternal stress [68]. Given this study, it should be investigated if prenatal CSE exerts similar effects on the HPA-axis.

SIRT1 is a NAD+-dependent enzyme that deacetylates proteins [255]. SIRT1 activity is induced through the sensing of low energy status through levels of $\mathrm{NAD}^{+} / \mathrm{NADH}$ to induce increased deacetylation of various transcription factors [255]. This deacetylation action results in the induction of gluconeogenesis, fatty acid oxidation, as well as the suppression of lipogenesis [255]. Conversely, the high energy status observed during a fed state, or consumption of a highfat diet, results in lower levels of $\mathrm{NAD}^{+}$and thus lowers the activity of SIRT1 [255]. Placental samples showing IUGR gathered from patients experiencing preeclampsia exhibited decreased expression of Sirt1 [294] but appears to be unchanged in the fetal liver in a sheep model of placental 
insufficiency IUGR [295]. A model using in utero calorie restriction of Wistar rats found that when 21-day-old male offspring but not female offspring exhibited lower levels of hepatic Sirt1 expression [296]. In a Sprague Dawley rat model of in utero food restriction, low birth weight offspring exhibited increased prenatal hepatic SIRT1 activity with decreased hepatic SIRT1 activity at one day and three months of age [297]. Increased levels of histone $\mathrm{H} 3$ acetylation have been found in the livers of 21 day-old rat offspring subjected to IUGR [298]. Considering how both nutrient restriction in utero, as well as IUGR, modulate SIRT1 expression and their similarity in birth outcomes to in utero cigarette smoke exposure, it is pertinent to examine the role SIRT1 plays in prenatal cigarette smoke exposure. A study conducted by our lab utilizing murine in utero and postnatal exposure to cigarette smoke observed that exposed offspring exhibited decreased birth weight with persistent weight decrements and decreased hepatic SIRT1 expression at six months of age [40]. This provides rationale for further investigating the role of SIRT1 in mediating the impact of in utero cigarette smoke exposure as well as whether it influences weight gain from high-fat diet consumption in adulthood.

In this study, we utilized an established mouse inhalation model of cigarette smoke exposure that mimics active maternal smoking during pregnancy spanning from preimplantation throughout organogenesis and into late-stage fetal maturation (GD6-GD19). This period would be approximately gestational day 13.5 to approximately the end of the first trimester in humans [299]. In terms of liver development, this would correspond to liver development through hepatoblast differentiation into hepatocytes and cholangiocytes in both mice and humans, and the beginning of bile duct formation in mice [168]. We characterized the impact CSE has on model phenotypic characteristics, including hepatic protein expression of key metabolic enzymes, including SIRT1 following a high-fat diet challenge. We focused on impacts on the liver due to the critical role it plays in regulating metabolic processes including glucose and lipid metabolic pathways. This study was conducted to shed light on the impact of prenatal CSE-induced perturbation of SIRT1associated liver metabolic regulatory pathways.

\section{Methods}




\section{Animal Exposure}

Female C57BL/6 mice maintained on LabDiet 5015 were mated overnight (two females to one male) and checked the following day for the presence of a vaginal plug. Vaginal sperm positive status was considered gestational day (GD) 0 . On GD6, female C57BL/6 mice were placed into either a cigarette smoke exposure (CSE) chamber or a Sham exposure (ambient filtered room air) chamber for six hours/day daily on GD1 through GD19. Before exposure, each day, fresh cages with approximately 2-3 grams of food and unlimited water were provided for the duration of the exposure. Whole-body cigarette smoke exposure was carried out utilizing a Teague TE-10C exposure apparatus (Teague Industries) using a standardized FTC method of smoking one 2-second puff per minute, nine puffs per cigarette. Two packs of cigarettes were used per day. The cigarettes used were Marlboro Red ${ }^{\mathrm{TM}}$, which was the most commonly smoked cigarette in reproductive-aged women at the time of model development. In order to quantify the composition of the cigarette smoke achieved, levels of $\mathrm{CO}$ and total suspended particulates (TSPs) were measured daily in each exposure chambers. Plasma cotinine was used as a measure of the internalization of cigarette smoke components since it is the primary metabolite of nicotine.

After parturition, dams with litters were maintained on LabDiet 5015 containing $12 \%$ fat. After weaning at three weeks of age, the offspring were maintained on the same diet until six months of age, at which age offspring entered the diet challenge. For the diet challenge, Sham and CSE offspring were assigned to maintenance on a low-fat control diet (CD, TestDiet 5TJS) or a high-fat diet (HFD, TestDiet 5TJN). The CD and HFD contained $5.1 \%$ and $19.9 \%$ fat, respectively. Other notable differences between diets include $70.1 \%$ carbohydrate, $15.5 \%$ protein, and $5.7 \%$ fiber content in the low-fat control diet versus $51.3 \%$ carbohydrate, $18.0 \%$ protein, and $6.7 \%$ fiber content in the high-fat diet. Offspring were maintained on these diets with unrestricted access for three months. Offspring weights were recorded weekly. During the diet challenge, selected offspring from the same prenatal exposure group were cohoused. No more than two animals of the same sex were cohoused together in a single cage during the duration of the diet challenge. At the end of the diet challenge, offspring were euthanized following a 4-hour 
fast by asphyxiation by carbon dioxide. The excised liver was flash-frozen in liquid nitrogen and stored at $-80^{\circ} \mathrm{C}$.

Animals were housed and maintained at the University of Louisville Research Resources Center, an Association for Assessment and Accreditation of Laboratory Animal Care accredited facility. All protocols were approved by the UofL Institutional Animal Care and Use Committee. Cages for animal housing were changed weekly, unlimited food and water provided, and maintained in an environment with a 12-hour light/dark cycle with controlled temperature and humidity.

\section{Characterization of Offspring Body Weight, Calorie Consumption, Fat Mass, and General Activity Levels}

Body weight and food consumption of offspring were measured once a week during the 3-month diet challenge. The body weight for each offspring was measured while food consumption was recorded on a per cage basis. The first week of body weight and food consumption was excluded from analyses to avoid the effects of acclimation to a new diet from confounding the statistical analyses. Offspring fat mass was measured via Dual-Energy X-Ray Absorptiometry (DEXA) using the Lunar PIXImus Densitometer (GE Medical Systems).

Within one week before the termination of the diet challenge, offspring (aged nine months) maintained on a high-fat diet were placed into a clean open arena and allowed to move freely for a trial time of 30 minutes with movement recorded by video. The video of each open field trial was analyzed utilizing open-source MATLAB-based software developed for the automation of the scoring of various behavioral assays [300]. Movement of individual mice during the trials was tracked in order to calculate the total distance traveled, speed, and as a measure of anxiety, the time spent in the inner versus the periphery of the arena.

\section{Cigarette Smoke Exposure Monitoring via Plasma Cotinine Measurement, Carbon Monoxide, and Total Suspended Particulates}


In order to quantitate the magnitude of cigarette smoke dams were exposed to, cotinine, the primary metabolite of nicotine, was measured during the cigarette smoke exposure period. Tail vein blood was collected from dams within 30 minutes of the end of exposure into heparinized capillary tubes and was subsequently centrifuged at $10,000 \times g$ for 10 minutes. The collected plasma fractions were stored at $-20^{\circ} \mathrm{C}$ until ready for cotinine measurement. Using the Cotinine Direct Elisa Kit (Immunalysis, Pomona CA), the collected plasma fractions were assayed for the concentration of cotinine following the manufacturer's instructions. Data was reported as nanograms per milliliter. Levels of plasma cotinine greater than or equal to $50 \mathrm{ng} / \mathrm{mL}$ were considered sufficient to simulate active maternal smoking during pregnancy. Twice daily, the level of $\mathrm{CO}$ within each exposure chamber was recorded on a carbon monoxide detector with a digital readout in parts per million. TSPs were recorded twice daily by drawing air from each exposure chamber via a sampling port through a preweighed piece of filter paper. Each piece of filter paper was then reweighed, and the calculated difference pre- and post-sampling was divided by the volume of air that was drawn in an interval of five minutes while sampling each chamber.

\section{Western Blotting}

Approximately 0.3 grams of liver was homogenized in $500 \mu \mathrm{L}$ of ice-cold buffer [100 mM PO4 Buffer, $150 \mathrm{NaCl}, 0.1 \%$ Tween, 0.1\% SDS, 0.5M Tris pH 8.0, 2 tabs per $10 \mathrm{~mL}$ phosphatase and protease inhibitor cocktails, $10 \mu \mathrm{L}$ per $10 \mathrm{~mL}$ EDTA, $7.5 \mathrm{mg} / \mathrm{mL}$ dithiothreitol (DTT)] using a Tissue-Tearor homogenizer in $2 \mathrm{sec}$ on/off pulses over the span of 1 minute. The sample was kept on ice during homogenization. The concentration of protein in each sample was measured by the Bradford method [301]. Liver homogenates $(1000 \mu \mathrm{g}$ of total protein per sample in a total volume of $240 \mu \mathrm{L})$ were mixed with Laemmli buffer $(0.25 \mathrm{M}$ Tris $\mathrm{pH} 6.8$, glycerol, $10 \%$ SDS, trace bromophenol blue), reduced with DTT $(1 \mathrm{~g} / 5 \mathrm{~mL})$ then heated at $70^{\circ} \mathrm{C}$ for 10 minutes for a final concentration of $4.2 \mu \mathrm{g} / \mu \mathrm{L}$. Thirty-five $\mu \mathrm{g}$ total protein was applied to $10 \%$ acrylamide gels with separation at 100V ( 2hours) in Tris-glycine run buffer (25 mM Tris, $192 \mathrm{mM}$ glycine, pH 8.3) followed by transfer to PVDF membrane at 70V for 2 hours in non-reducing buffer (Tris-Glycine 
buffer; 25 mM Tris; 192 mM glycine, pH 8.3 with 20\% ethanol). Following blocking in 5\% non-fat dry milk in TBS-T solution (Tris-buffered Saline-Tween; $137 \mathrm{mM} \mathrm{NaCl}, 20 \mathrm{mM}$ Tris-Base pH 7.6, $0.1 \%$ Tween-20), the blots were incubated overnight at $4^{\circ} \mathrm{C}$ with primary antibody (SIRT1 Santa Cruz \#sc-15404, 1/500 dilution; PEPCK Santa Cruz \#sc-271029, 1/500 dilution; GAPDH Cell Signaling \#5174S, 1/5000 dilution; NAMPT Thermo-Fisher \#PA5-23198, 1/1000 dilution; SULT2A1 Thermo-Fisher \#PA5-12243, 1/1000 dilution; CYP8B1 Thermo-Fisher \#PA5-37088, 1/1000 dilution; Catalase Cell Signaling \#14097, 1/5000 dilution; SOD2 Cell Signaling \#13194, 1/1000 dilution) diluted in non-fat dry milk in TBS-T. After three 10-minute washes in TBS-T, blots were incubated with secondary antibody complexed to horseradish peroxidase (anti-mouse, Thermo \#32430; anti-rabbit, Cell Signaling \#7074S) in 5\% non-fat dry milk diluted in TBS-T at room temperature for 3 hours. After three successive washes in TBS-T, blots were developed with LumiGLO chemiluminescent reagent and peroxide (Cell Signaling Technology \#7003S, Danvers, MA), and visualized with a Bio-Rad Gel Doc XR+ system (Bio-Rad Laboratories, Hercules, CA). Following visualization, blots were washed briefly in TBS-T and incubated with Coomassie stain (0.1\% Coomassie Brilliant Blue G250 Dye in 1:1 methanol:water) for 10 minutes, followed by two 10 minute washes in destaining solution (5:4:1 ethanol:water:acetic acid), and a final wash for 5 minutes in deionized water. Blots were briefly air-dried, placed on a scanner, then scanned into a digital format for import into ImageJ. Quantification of total protein staining and target protein bands was performed in ImageJ by measuring pixel density for each sample. The expression of the target protein was normalized per sample by the pixel density of the total protein stain for each sample.

\section{Liver Triglyceride Assay}

Liver tissue (approximate weight of 0.10 grams) was incubated in $350 \mu \mathrm{L}$ of ethanolic $\mathrm{NaOH}$ at $55^{\circ} \mathrm{C}$ overnight with periodic vortexing. The following morning, sample volume was adjusted to $1000 \mu \mathrm{L}$ with 1:1 $\mathrm{H}_{2} \mathrm{O}: 70 \%$ reagent grade $\mathrm{EtOH}$ then centrifuged for 5 minutes at $4500 \mathrm{~g}$. The supernatant was placed into a new tube, diluted to $1200 \mu \mathrm{L}$ with $1: 1 \mathrm{H}_{2} \mathrm{O}: 70 \%$ reagent grade $\mathrm{EtOH}$ and vortexed. Two hundred microliters of the diluted supernatant were 
mixed with $215 \mu \mathrm{L}$ of $\mathrm{MgCl}_{2}$. After incubating on ice for 10 minutes, the sample was centrifuged for 5 minutes, followed by placing the supernatant into a new tube.

Six microliters of sample or glycerol standard (Sigma \#G7793) were mixed with $194 \mu \mathrm{L}$ of free glycerol reagent (Sigma \#F6428) and assayed with free glycerol reagent used as a blank. The microtiter plate was incubated at $37^{\circ} \mathrm{C}$ for 15 minutes, and then absorbance was read at 540 $\mathrm{nm}$. Triolein equivalents in samples were calculated as $\mathrm{mg}$ triglyceride/gram tissue.

\section{Linear Mixed-Effects Modeling}

Linear mixed-effects modeling using the Ime4 $\mathrm{R}$ package [302] was conducted to assess the independent and synergistic effects of prenatal CSE, postnatal adult diet, and sex of the offspring on phenotypic characteristics and hepatic protein expression levels. Repeated measures analysis was also incorporated. The general equation utilized to model the repeated measures data is given by:

\section{Eq. 1: Variable of Interest $\sim 1+$ Week ${ }^{*}$ Sex ${ }^{*}$ Exposure ${ }^{*}$ Diet + (Week|Litter/Individual Offspring $)+($ Week|Litter:Sex $)+($ Week|Litter:Diet $)$}

Where Week*Sex*Exposure*Diet indicates modeling of fixed effects for the week of diet challenge, sex of the offspring, prenatal exposure, and postnatal diet. All possible interactions between the four fixed effects were considered with offspring treated as individuals. The (Week|Litter/Individual Offspring) term indicates the weeks of the diet challenge set as a random slope and the identity of individual offspring within each litter set as a random intercept. The random intercept was set this way to account for the fact that each individual offspring is more likely to be similar to other littermates than offspring from different litters. The goal of this was to help control potential confounding of results due to potential natural variance between litters. The Week variable was set as a random slope to account for the fact that the rate of change from one week to the next may be variable and not constant. For the (Week|Litter:Sex) and (Week|Litter:Diet) terms, the random intercept was modeled as a separate random litter intercept 
per sex and diet. These additional random intercept terms were used to account for potential baseline differences due to the litter that offspring belong to with respect to the sex of the offspring and what diet they were maintained on. The general equation used for endpoint data (body weight and total calories consumed at the end of the diet challenge, offspring activity, body fat tissue percentage, liver triglyceride assay, and hepatic protein expression) is given by:

Eq. 2: Variable of Interest $\sim 1+$ Sex*Exposure*Diet $+(1 \mid$ Litter $)+(1 \mid$ Litter:Sex $)+(1 \mid$ Litter:Diet $)$

The above equation 2 is similar to 1 but without the Week variable. For each analysis conducted, there were issues with fitting random effects models due to the occurrence of singular fits. For the general models listed above, random effects terms were systematically removed until each model successfully converged and no longer resulted in singular fits. Any non-significant random effects, interaction terms, or fixed effects were systematically tested for using ANOVA and removed using $\mathrm{AIC}$ and $\mathrm{BIC}$ as the criteria for a good model fit to the data. After this process the most parsimonious model was considered finalized for downstream analysis. Post-hoc testing was conducted using t-tests for specific a priori contrasts of interest using the finalized models with the emmeans R package [303] in which multiple testing was corrected for using the HolmBonferroni method [304]. One of the sets of contrasts tested for was the effect of CSE versus Sham exposure: the effect of CSE versus Sham within Male offspring on a Control Low-fat Diet, Male offspring on a High-fat Diet, Female offspring on a Control Low-fat Diet, and Female offspring on a High-fat Diet. The second set of contrasts tested for the effect of High-fat Diet versus Control Low-fat Diet: High-fat Diet vs Control Low-fat Diet within Male Sham offspring, Male CSE offspring, Female Sham offspring, and Female CSE offspring. The last set of contrasts tested for the effect of the sex of the offspring: Female versus Male offspring within Sham offspring on a Control Low-fat Diet, Sham offspring on a High-fat Diet, CSE offspring on a Control Low-fat Diet, and CSE offspring on a High-fat Diet. These sets of contrasts were chosen since they were salient to the hypotheses to be tested. The first week and the first two weeks of 
the weight and calorie consumption data, respectively, were removed to control for acclimation effects to new diets.

\section{Results}

\section{Cigarette Smoke Exposure Conditions and Birth Weight of Offspring}

The carbon monoxide level in the cigarette smoke exposure chamber was $110.88 \mathrm{ppm}+/-$ $8.096 \mathrm{ppm}$ while total suspended particulates averaged $29.14 \mathrm{mg} / \mathrm{m}^{3}+/-8.34 \mathrm{mg} / \mathrm{m}^{3}$. These values are consistent with previous cigarette smoke exposures performed by the Neal and Pisano labs (138 ppm +/- $19.8 \mathrm{ppm} \mathrm{CO}$ and $25.4 \mathrm{mg} / \mathrm{m}^{3}+/-6.5 \mathrm{mg} / \mathrm{m}^{3} \mathrm{TSP}$ [40]; $128 \mathrm{ppm}+/-3.0 \mathrm{ppm} \mathrm{CO}$ and $29.2 \mathrm{mg} / \mathrm{m}^{3}+/-2.6 \mathrm{mg} / \mathrm{m}^{3} \mathrm{TSP}$ [41]). The values of each of these measures of CSE were below the limit of detection in the Sham chamber. Plasma cotinine levels in sperm positive females were $84.57 \mathrm{ng} / \mathrm{ml}+/-8.34 \mathrm{ng} / \mathrm{mL}$. Plasma cotinine exceeded $50 \mathrm{ng} / \mathrm{ml}$, one accepted cutoff to designate a woman as an active smoker [305]. The plasma cotinine levels in the Sham sperm positive dams were below the limit of detection of $4 \mathrm{ng} / \mathrm{mL}$. The CSE offspring exhibited a low birth weight phenotype with a $\sim 15 \%$ reduction in birth weight similar to that found in our prior studies [35-37]. Catch-up growth was evident in the CSE offspring by the initiation of diet challenge (Figure 2). Male and female offspring did not vary in weight on week one based on prenatal exposure. Offspring subjected to in utero cigarette smoke exposure exhibited the classic phenotype of low birth weight followed by subsequent catch-up growth as predicted by the Barker Hypothesis.

\section{CSE-Induced Offspring Body Weight Perturbations Modulated by Diet and Sex of Offspring}

As shown in Figure 2, throughout the 3-month diet challenge, male CSE offspring exhibited a trend toward weekly weight gain both on a low-fat control and a high-fat diet ( $p=0.06$ for both). Male offspring exhibited increased weight gain when maintained on a high-fat diet regardless of prenatal exposure $(p<0.05)$. Female offspring exposed to cigarette smoke during gestation exhibited a significant increase in body weight gain when maintained on a high-fat diet $(p<0.05)$ while no difference in weight was observed when female offspring exposed in utero to cigarette smoke were maintained on a low-fat control diet. Female offspring exhibited increased weight gain 


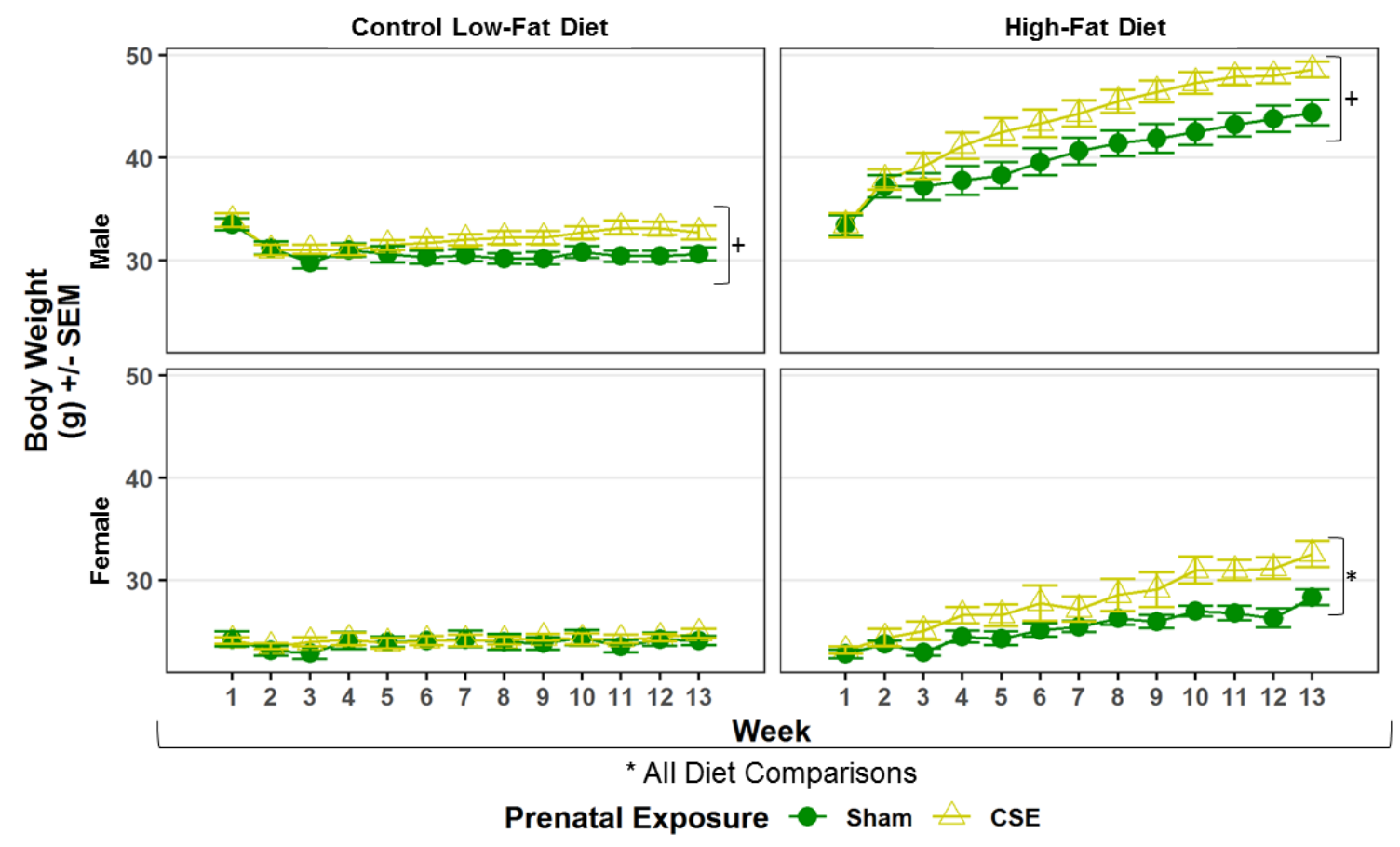

Figure 2 - CSE exacerbates diet-modulated sex-specific weight gain - The body weight of offspring was recorded weekly after the initiation of the diet challenge. Prenatal exposure to cigarette smoke exacerbated weight gain in female offspring maintained on a high-fat diet. Prenatal exposure to cigarette smoke resulted in a trend toward increased weight gain in male offspring when maintained on either diet. Maintenance of offspring on a highfat diet resulted in increased weight gain regardless of prenatal exposure or sex. Male offspring maintained on a high-fat diet gained weight more rapidly than female offspring on the same diet. ${ }^{*} p<0.05,+p<0.1$. 
when maintained on a high-fat diet regardless of prenatal exposure $(p<0.05)$. Male offspring gained weight more rapidly than female offspring when maintained on a high-fat diet $(p<0.05)$. There was not an evident difference in weight gain between male and female offspring when maintained on a low-fat control diet. There was no impact of prenatal exposure on weight gain between male and female offspring. When subjected to only in utero cigarette smoke exposure, female offspring appear resistant to the increased weight gain evident in male offspring, but this resistance was not observed when female offspring were maintained on a high-fat diet.

In Figure 3, at the end of the 12-week diet challenge period at nine months of age, maintenance of all male offspring on a high-fat diet resulted in a significant increase in body weight gain regardless of prenatal exposure $(p<0.05)$. Male offspring exposed in utero to cigarette smoke and then maintained on a high-fat diet exhibited a trend toward increased body weight $(p=0.06)$. Male CSE offspring maintained on a control low-fat diet exhibited no difference in body weight. Female CSE offspring maintained on a high-fat diet exhibited a significant increase in body weight compared to female Sham offspring maintained on a high-fat diet $(p<$ 0.05). There was no impact of prenatal CSE on body weight in female offspring maintained on a low-fat control diet. Female CSE offspring on a high-fat diet exhibited a significant increase in body weight compared to female CSE offspring maintained on control low-fat diet $(p<0.05)$. Sham female offspring maintained on a high-fat diet exhibited a trend toward increased body weight compared to Sham female offspring maintained on a low-fat control diet $(p<0.10)$. In all instances, male offspring were significantly heavier than female offspring regardless of prenatal exposure or diet $(p<0.05)$. The manifestation of the Barker Hypothesis in female offspring subjected to in utero cigarette smoke exposure was conditional on the composition of the diet in adulthood. These female offspring outcomes were in contrast to the consistent manifestation of the Barker Hypothesis in the male offspring exposed in utero to cigarette smoke regardless of the diet they were maintained on in adulthood.

Impact of Prenatal Cigarette Smoke Exposure and Adult Diet Challenge on Calorie Consumption and Measures of Offspring Activity 


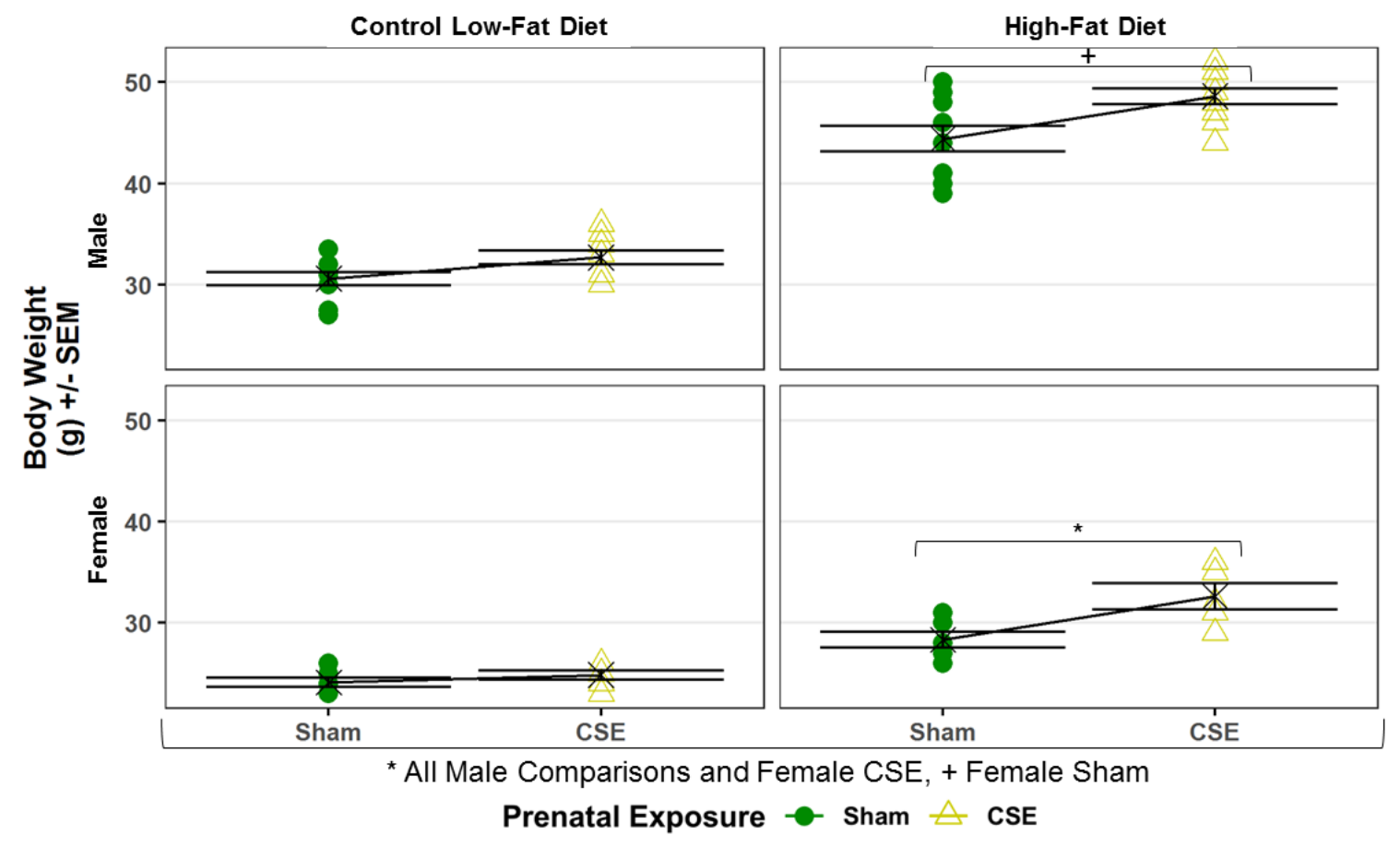

Figure 3 - CSE-induced increase in sex-specific weight and modulated by diet - By the end of the 12-week diet challenge, all offspring maintained on a high-fat diet exhibited increased weight as compared to those offspring maintained on a low-fat control diet, except the female Sham offspring. When maintained on a high-fat diet, male offspring subjected in utero to cigarette smoke resulted in a trend toward increased weight, whereas the corresponding female offspring exhibited a significant increase in weight. In all instances, male offspring were heavier than female offspring. ${ }^{*} p<0.05$. 
As shown in Figure 4, the average total calories (kcals) consumed per animal during the 12-week diet challenge did not differ based on prenatal exposure or sex of the offspring. Consumption of a high-fat diet, however, resulted in significantly more kcals consumed by both male and female offspring as compared to male and female offspring maintained on a control diet $(p<0.05)$. The calories consumed weekly were monitored during the 12-week diet challenge (Figure 5). Male offspring exposed in utero to cigarette smoke and maintained on a high-fat diet, at the time of diet challenge at age six months, exhibited lower weekly consumption of diet over time compared to male Sham offspring maintained on a high-fat diet $(p<0.05)$. There was no impact of prenatal CSE on weekly consumption in male offspring maintained on a low-fat control diet. There was no impact of a high-fat diet on weekly consumption in male offspring. Female offspring exposed in utero to cigarette smoke and maintained on a high-fat diet exhibited lower weekly consumption of diet over time compared to female Sham offspring maintained on a high-fat diet $(p<0.05)$. Female Sham offspring maintained on a high-fat diet consumed fewer calories over time compared to female Sham offspring maintained on a low-fat control diet $(p<0.05)$. Female offspring maintained on a high-fat diet consumed more calories over time compared to male offspring when maintained on a high-fat diet ( $\mathrm{p}$ 0.05). It should be noted that in Figure 5 , the magnitude of all significant differences amounted to less than $1 \mathrm{kcal} /$ week. Overall, the one notable change observed in calorie consumption was the greater total calories consumed by offspring maintained on a high-fat diet versus a low-fat control diet during the entire 12-week diet challenge. Although there were minor differences as a result of prenatal exposure to cigarette smoke, it seems that calorie consumption did not have a meaningful impact on prenatal CSEinduced weight gain observed in exposed offspring.

One potential mediator of offspring body weight differences, general activity level, was assessed by open-field activity in the offspring. Only the offspring maintained on a high-fat diet underwent open-field testing. As shown in Figure 6A, prenatal CSE did not affect the speed of offspring maintained on a high-fat diet compared to Sham offspring maintained on a high-fat diet. Female Sham offspring maintained on a high-fat diet exhibited a trend toward increased activity 


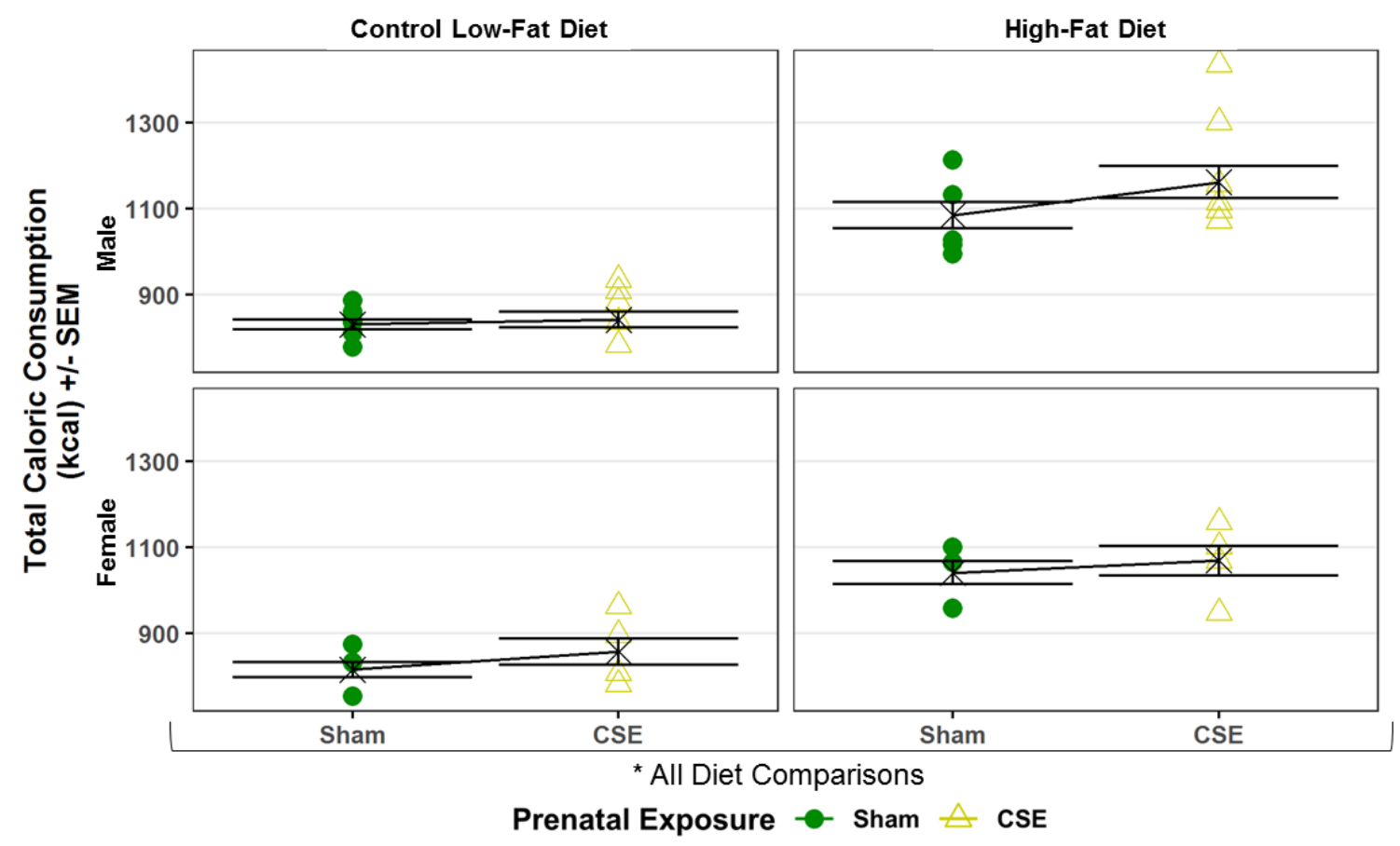

Figure 4 - High-fat diet results in greater calorie consumption - The number of calories (kcal) consumed throughout the diet challenge was monitored. Neither sex of the offspring nor prenatal exposure influenced the total amount of calories consumed for the duration of the diet challenge. All offspring that were fed a high-fat diet consumed more total calories than those offspring maintained on a low-fat control diet. ${ }^{*} p<0.05$. 


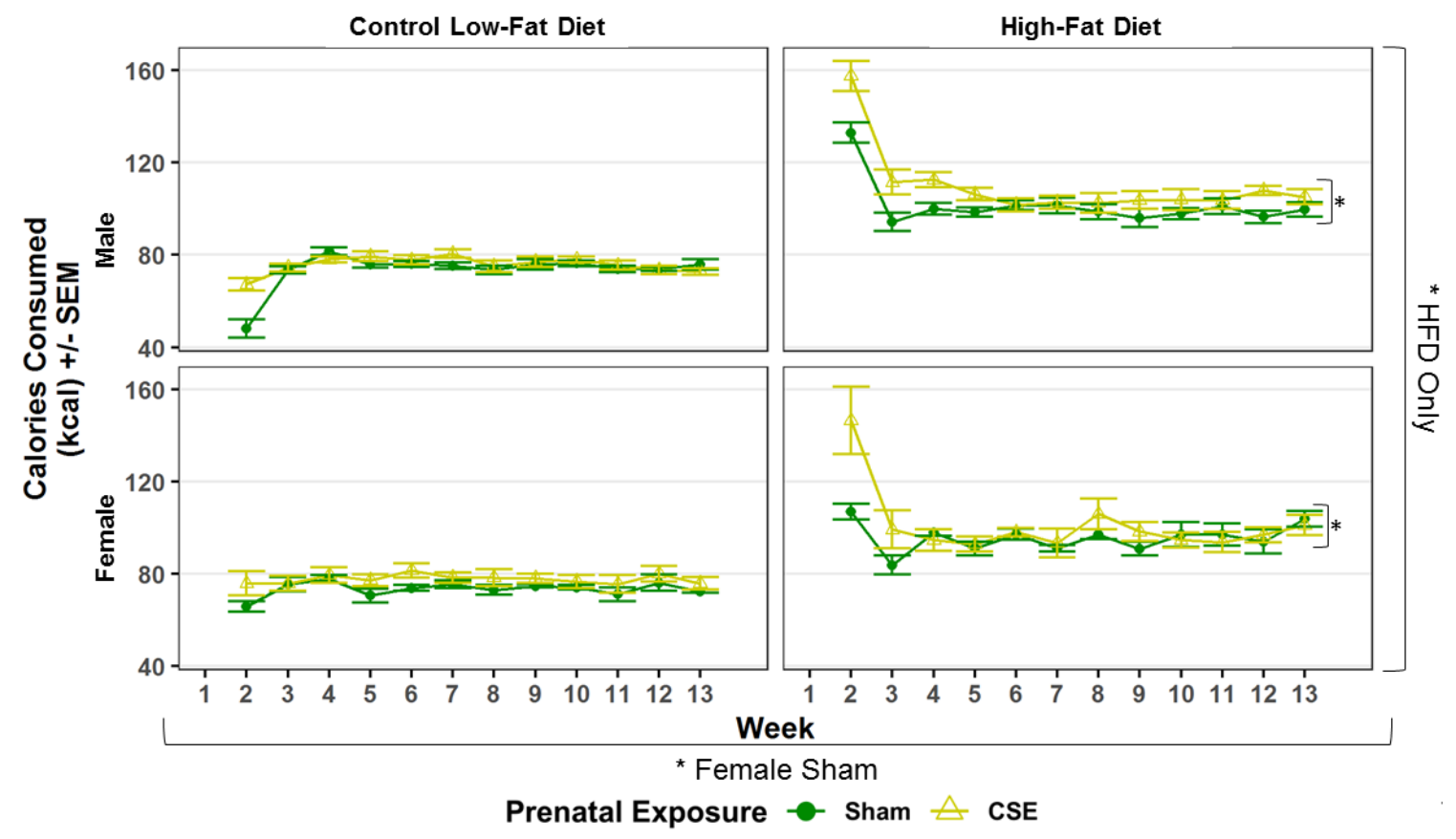

Figure 5 - Minor effect of CSE on the rate of calorie consumption modulated by diet - The number of calories (kcal) consumed weekly were tracked throughout the diet challenge. Week 1 was excluded to allow for acclimation to a new diet. When maintained on a high-fat diet, offspring with prenatal exposure to cigarette smoke consumed calories at a lower rate. Female offspring fed a high-fat diet consumed more calories at a higher rate than male offspring maintained on a highfat diet. Female offspring fed a high-fat diet consumed calories at a higher rate than female offspring maintained on a low-fat control diet. All effect sizes were $<1$ calorie/week. ${ }^{*} p<0.05$. 


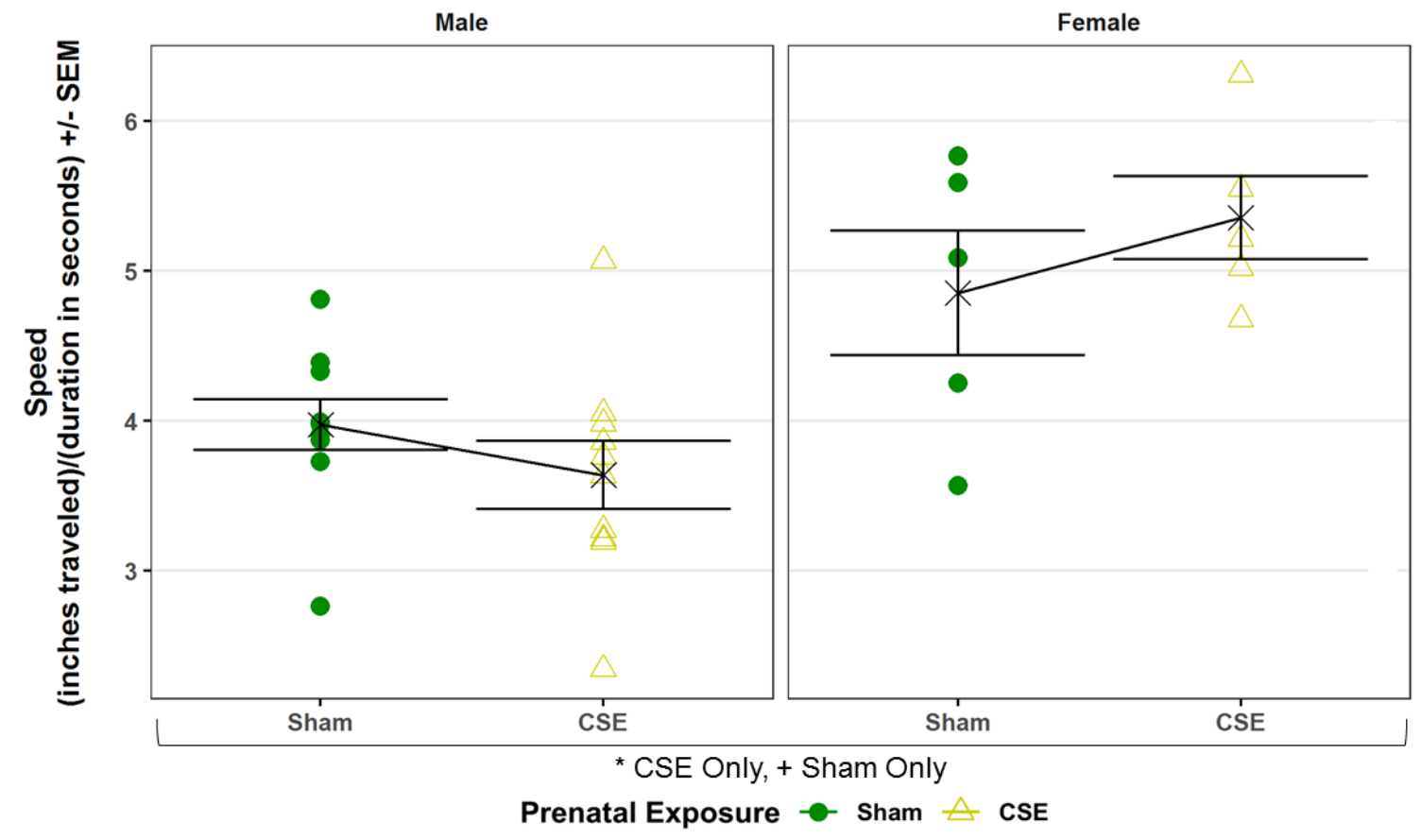

Figure 6A - Female CSE offspring traveled more quickly on HFD than male CSE offspring -

Open field assessment was used to determine the average speed of individual offspring while traveling in the arena. Female offspring exposed in utero to cigarette smoke and maintained on a high-fat diet were more active than male CSE offspring maintained on a high-fat diet. Female Sham offspring maintained on a high-fat diet resulted in a trend toward increased activity compared to the male Sham offspring maintained on a high-fat diet. ${ }^{*} p<0.05,+p<0.1$. 
compared to male Sham offspring maintained on a high-fat diet $(p=0.08)$. Female CSE offspring maintained on a high-fat diet were significantly more active than male CSE offspring maintained on a high-fat diet $(p<0.05)$. As shown in Figure 6B, the distance each offspring traveled mirrored the speed each offspring traveled in Figure 6A. Female CSE offspring maintained on a high-fat diet traveled more than male CSE offspring maintained on a high-fat diet $(p<0.05)$. As shown in Figure 6B, female Sham offspring maintained on a high-fat diet exhibited a trend toward increased distance traveled compared to male Sham offspring maintained on a high-fat diet $(p=$ 0.07). In Figure $6 \mathrm{C}$, the percentage of trial duration spent in the inner region of the arena, a measure of anxiety where mice naturally try to minimize the time spent in the center of the arena, was increased in female CSE offspring maintained on a high-fat diet compared to female Sham offspring maintained on a high-fat diet $(p<0.05)$. Female CSE offspring maintained on a high-fat diet spent more of the trial in the inner region of the arena compared to male CSE offspring maintained on a high-fat diet $(p<0.05)$. The absence of effects of prenatal CSE on the level of activity suggests a lack of contribution to the increased weight gain observed in Figure 2 in offspring subjected to in utero cigarette smoke exposure.

\section{CSE-Induced Sex-Specific Effect on Offspring Adiposity is Modulated by Diet}

Body composition (lean and fat tissue mass, bone mass, and density) was assessed by DEXA scanning. As shown in Figure 7, male CSE offspring maintained on a low-fat control diet exhibited a trend toward an increased percentage of fat tissue compared to Sham male offspring maintained on the same diet $(p=0.06)$. Male CSE offspring maintained on a high-fat diet exhibited no difference compared to male Sham offspring maintained on a high-fat diet. Male offspring maintained on a high-fat diet exhibited increased body fat compared to male offspring maintained on a low-fat control diet regardless of prenatal exposure $(p<0.05)$. Female CSE offspring on a high-fat diet exhibited an increased percentage of body fat tissue compared to Sham female offspring maintained on a high-fat diet $(p<0.05)$. There was no impact of prenatal CSE on body fat in female offspring maintained on a low-fat control diet. Female offspring maintained on a high-fat diet exhibited increased body fat compared to female offspring 


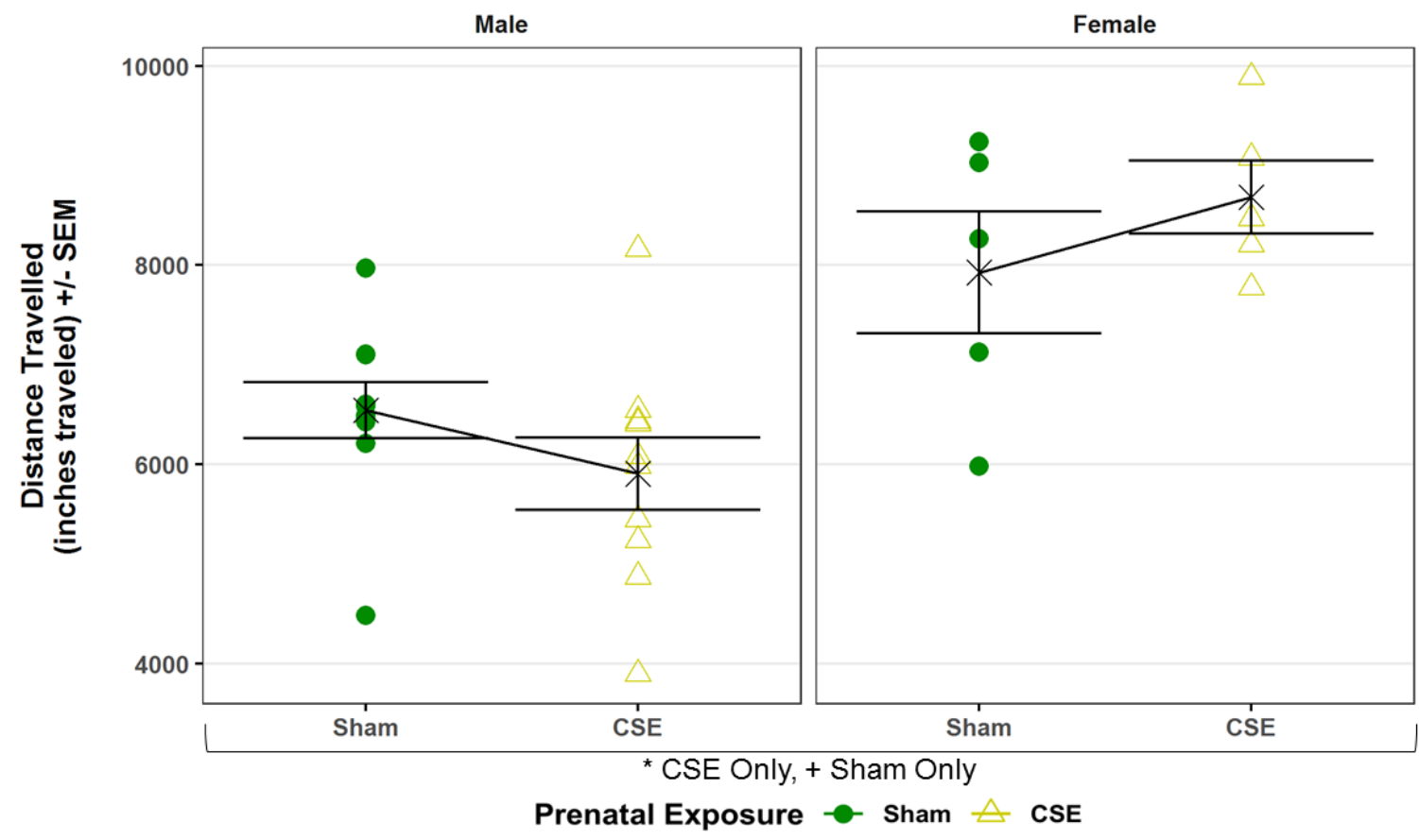

Figure 6B - Female CSE offspring traveled more on HFD than male CSE offspring - Open field assessment was used to determine the distance individual offspring traveled in the arena. Female offspring exposed in utero to cigarette smoke and maintained on a high-fat diet traveled more than male CSE offspring maintained on a high-fat diet. Female Sham offspring maintained on a high-fat diet resulted in a trend toward increased distance traveled compared to the male Sham offspring maintained on a high-fat diet. ${ }^{*} p<0.05,+p<0.1$. 


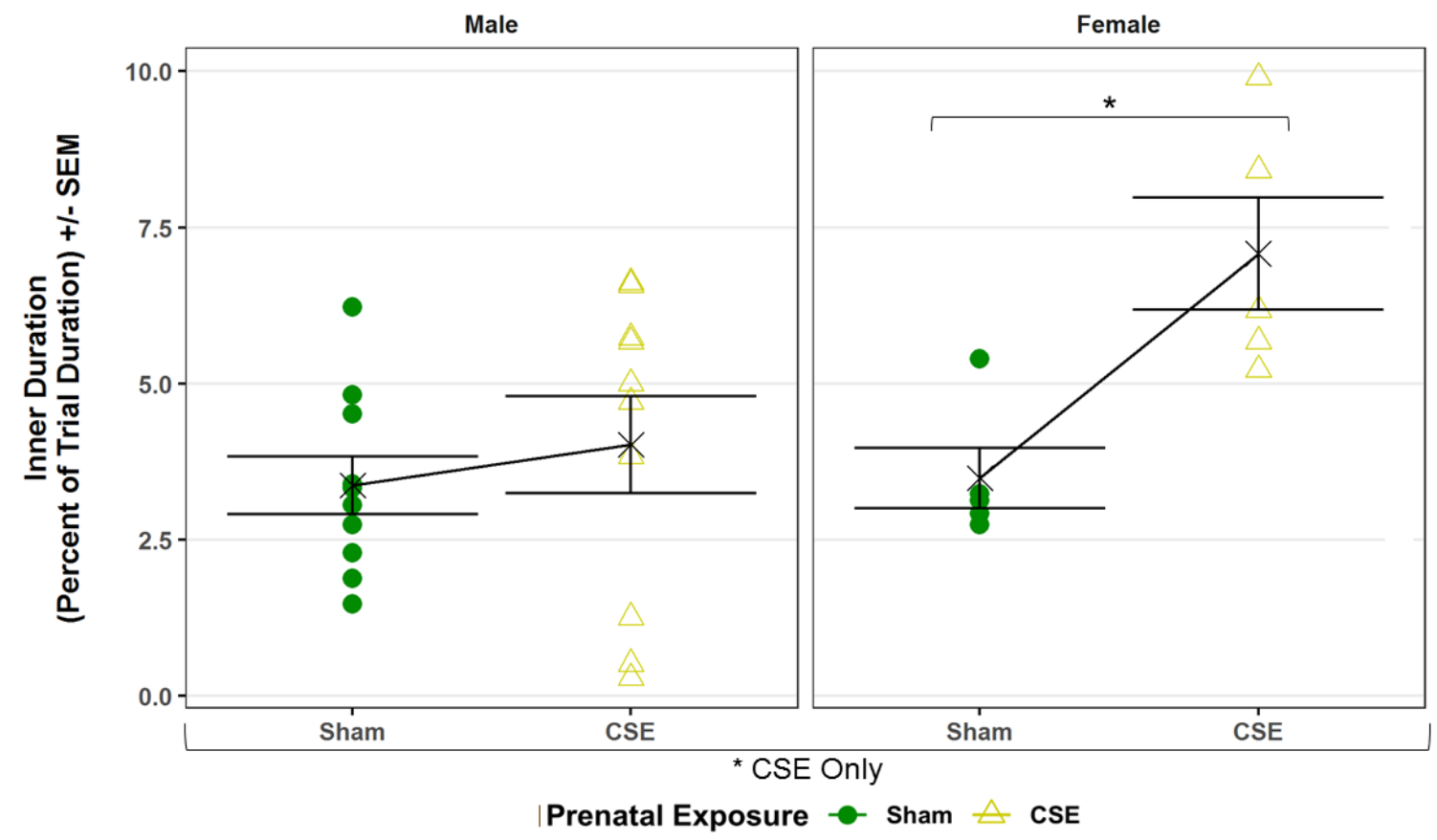

Figure 6C - Female CSE offspring on HFD display less anxious behavior than male CSE offspring - Open field assessment was used to determine the level of anxiety behavior in individual offspring. Female CSE offspring maintained on a high-fat diet spent a greater duration of the trial in the inner portion of the arena compared to female Sham offspring maintained on a high-fat diet, thereby displaying less anxiety behavior. Female offspring exposed in utero to cigarette smoke and maintained on a high-fat diet spent in the inner portion of the arena was increased compared to male CSE offspring maintained on a high-fat diet. ${ }^{*} p<0.05$. 


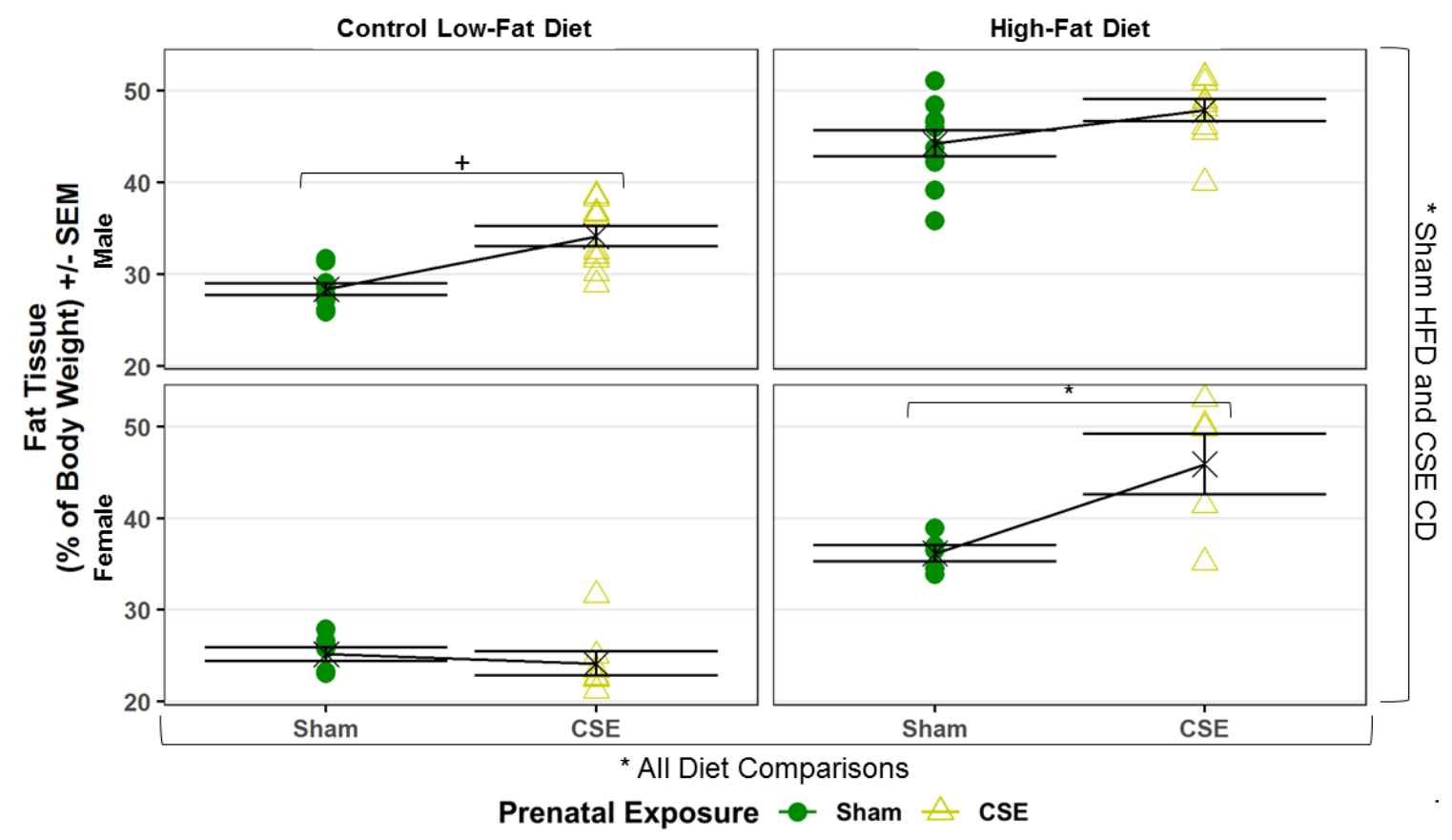

Figure 7 - Prenatal CSE increases body fat accumulation in female CSE offspring maintained on a HFD - DEXA scanning was used to characterize the body composition of the offspring, including fat, lean, and bone mass. Male offspring exposed in utero to cigarette smoke and maintained on a low-fat control diet resulted in a trend toward increased percentage body fat. An increase in percentage body fat was observed in female CSE offspring maintained on a highfat diet. A high-fat diet increased the percentage of body fat in all offspring regardless of prenatal exposure or sex of the offspring. Male CSE offspring maintained on a low-fat control diet had a greater percentage of body fat compared to female CSE offspring maintained on a low-fat control diet. Male Sham offspring maintained on a high-fat diet exhibited an increased percentage of body fat compared to female Sham offspring maintained on a high-fat diet. ${ }^{*} p<0.05,+p<0.1$. 
maintained on a low-fat control diet regardless of prenatal exposure. Sex had no impact on body fat Sham offspring maintained on a low-fat control diet. Female Sham offspring maintained on a high-fat diet exhibited a decreased percentage of body fat tissue compared to male Sham offspring maintained on a high-fat diet $(p<0.05)$. Female CSE offspring maintained on a control low-fat control diet exhibited a lower percentage of body fat tissue compared to male CSE offspring maintained on a control low-fat control diet $(p<0.05)$. Sex had no impact on body fat of CSE offspring maintained on a high-fat diet. It was not surprising that Sham offspring maintained on a high-fat diet for 12 weeks resulted in fat accumulation; what was surprising is the impact in utero cigarette smoke exposure had on fat accumulation in male Sham offspring maintained on a low-fat control diet, and female CSE offspring maintained on a high-fat diet. Our results demonstrated that in utero exposure to cigarette smoke in conjunction with maintenance on a high-fat diet caused exacerbated body fat accumulation in female offspring, mirroring the exacerbation of weight gain in female CSE offspring maintained on a high-fat diet.

Considering that maintenance on a high-fat diet can result in the development of fatty liver disease, liver triglycerides were measured in offspring at the end of the diet challenge. We anticipated that three months of being maintained on a high-fat diet should induce fatty liver in all male offspring as this is an established phenomenon [306-312]. As shown in Figure 8, liver triglycerides were significantly increased in all male offspring maintained on a high-fat diet compared to male offspring maintained on a control diet regardless of prenatal exposure $(p<$ 0.05). Prenatal CSE had no impact on liver triglyceride levels in offspring maintained on control low-fat diet. Neither prenatal exposure or diet had an impact on liver triglyceride levels in female offspring. There was no impact of the sex of offspring on liver triglyceride levels in offspring maintained on a low-fat control diet. Female offspring maintained on a high-fat diet had significantly lower levels of liver triglycerides compared to male offspring maintained on a high-fat diet regardless of prenatal exposure $(p<0.05)$. Despite the exacerbation of weight gain and body fat accumulation seen in female CSE offspring maintained on a high-fat diet, they appeared to be resistant to developing liver lipid accumulation, suggesting an innate resistance of female offspring to fatty liver development. It was evident for male offspring that in utero exposure to 


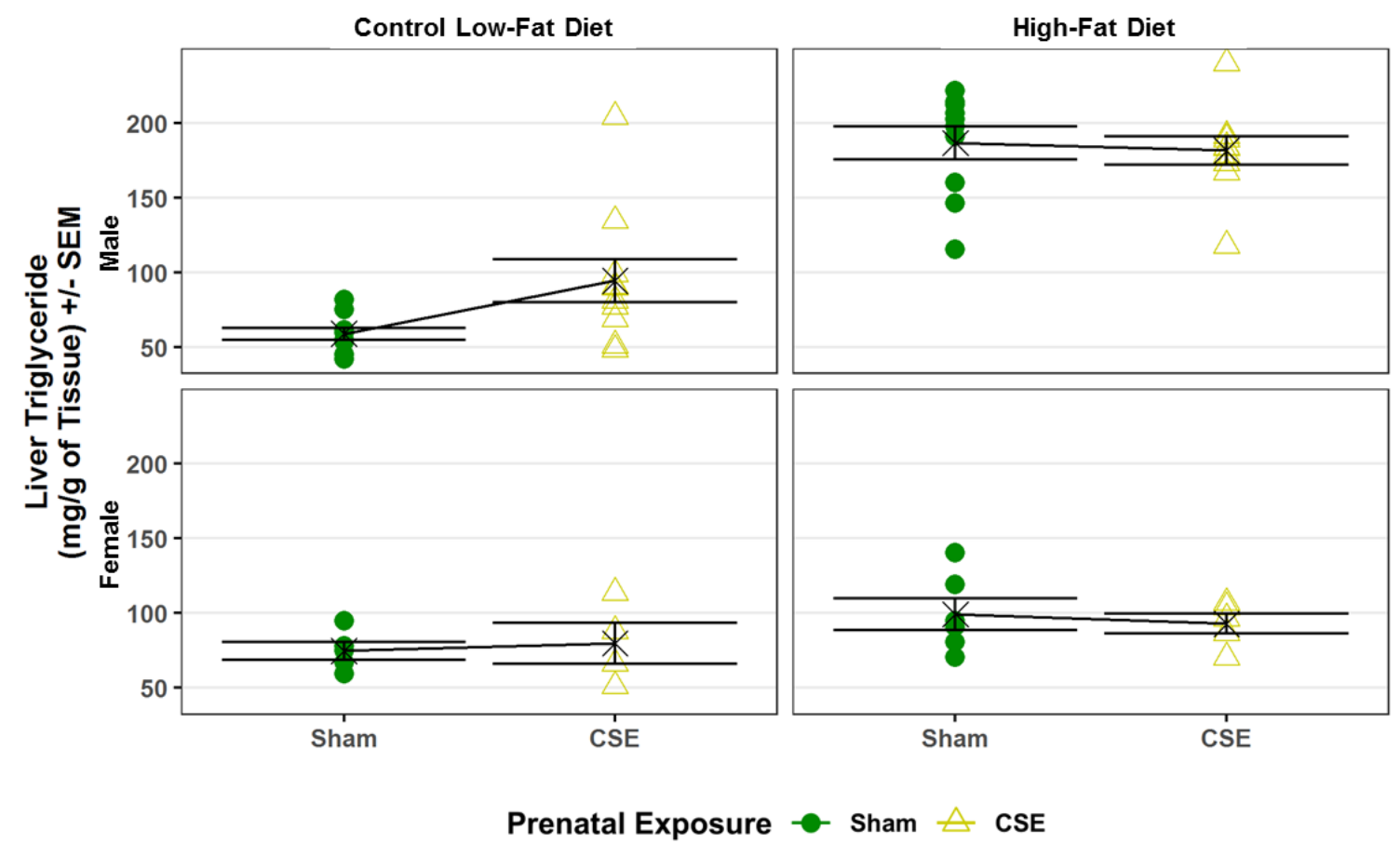

Figure 8 - Male offspring maintained on a low-fat diet do not exhibit liver fat deposition in response to prenatal CSE - Total liver triglycerides were measured from the livers of offspring. Prenatal exposure to cigarette smoke did not affect liver triglyceride concentrations. Male offspring maintained on a high-fat diet had significantly higher liver triglyceride levels than male offspring fed a low-fat control diet. Male offspring maintained on a high-fat diet had significantly higher liver triglyceride levels than the female offspring maintained on a high-fat diet. Female offspring were resistant to liver fat deposition. ${ }^{*} p<0.05$. 
cigarette smoke did not exert an additional impact on high-fat diet-induced fatty liver.

\section{CSE-Induced Changes in Hepatic Protein Expression of SIRT1-Related Enzymes are Modulated by Diet}

As shown in Figure 9A, hepatic SIRT1 protein expression is lower in male CSE offspring maintained on a low-fat control diet compared to male Sham offspring maintained on a low-fat control diet $(p<0.05)$. Prenatal CSE had no impact on SIRT1 expression in male offspring maintained on a high-fat diet. High-fat diet feeding in male offspring resulted in decreased hepatic protein expression of SIRT1 regardless of prenatal exposure $(p<0.05)$. Hepatic SIRT1 expression in female offspring was unaffected by either prenatal exposure or diet. In males, prenatal CSE followed by low-fat diet maintenance results in a similar reduction in hepatic SIRT1 when maintained on a high-fat diet.

Figure 9B shows the hepatic expression of NAMPT, an enzyme responsible for generating $\mathrm{NAD}^{+}$, a necessary cofactor for many cellular processes, including SIRT1 deacetylase activity. Hepatic expression of NAMPT in male offspring is unimpacted by either prenatal exposure or diet. In female offspring, the hepatic expression of NAMPT was unaffected by prenatal CSE when maintained on either diet. Female offspring maintained on a high-fat diet exhibit increased hepatic expression of NAMPT compared to female offspring maintained on a low-fat control diet regardless of prenatal exposure $(p<0.05)$. It was anticipated that the hepatic expression of NAMPT would be decreased in response to high-fat diet maintenance having been maintained on a high-fat diet for 12-weeks, but unexpectedly male offspring were unaffected while female offspring exhibited increased NAMPT expression

The hepatic expression of GAPDH, an essential regulatory enzyme in glycolysis, which is downregulated by SIRT1 through the deacetylation of PGC-1a, is shown in Figure 10A. In male CSE offspring maintained on a low-fat control diet, hepatic GAPDH expression was decreased compared to male Sham offspring maintained on a low-fat control diet $(p<0.05)$. Hepatic GAPDH expression was increased in male CSE offspring maintained on a high-fat diet compared to male Sham offspring maintained on a high-fat diet $(p<0.05)$. High-fat diet maintenance in 
Male
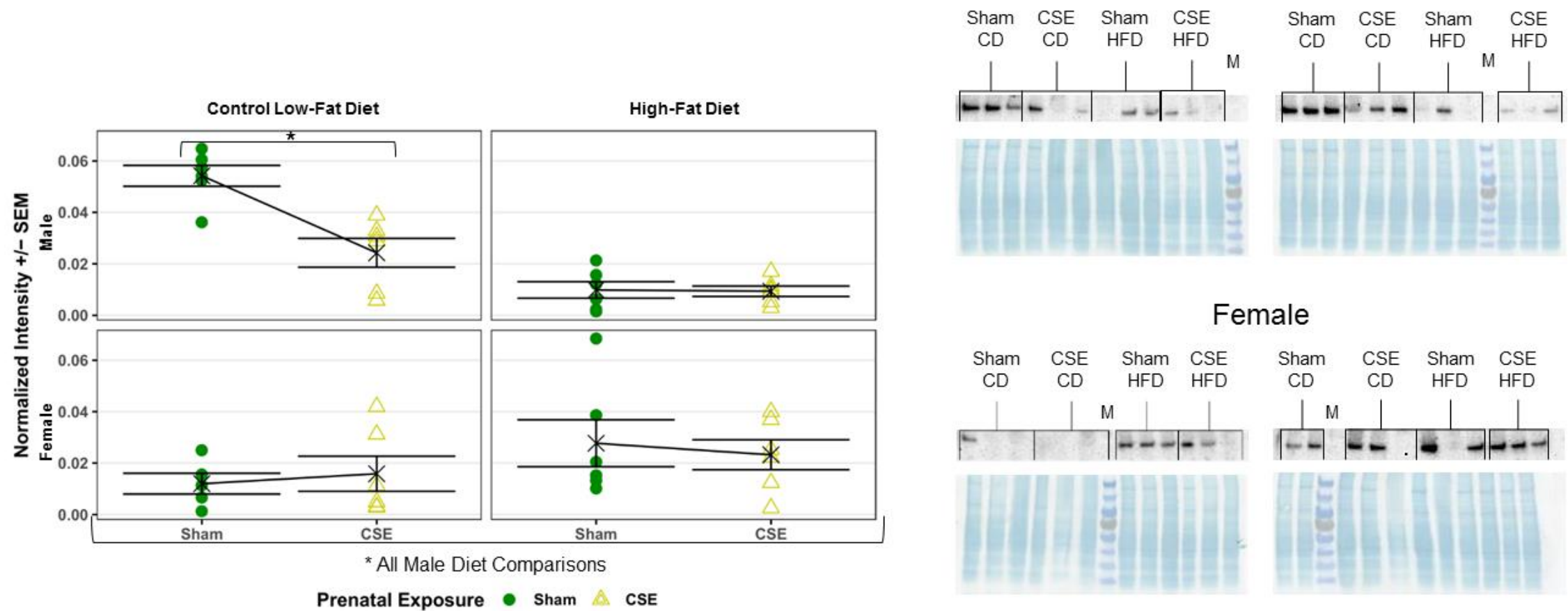

Figure 9A - Prenatal CSE or high-fat diet independently decrease hepatic SIRT1 protein expression in male offspring - Male CSE offspring maintained on low-fat control diet exhibited decreased hepatic SIRT1 expression compared to male Sham offspring maintained on a low-fat control diet. Male offspring maintained on a high-fat diet exhibited decreased hepatic SIRT1 expression compared to male offspring maintained on a lowfat control diet regardless of prenatal exposure. In male offspring, maintenance on a high-fat diet resulted in decreased expression of SIRT1 irrespective of prenatal exposure. There was no observed difference in hepatic SIRT1 expression in female offspring regardless of either prenatal exposure or diet. ${ }^{*} p<0.05$. 

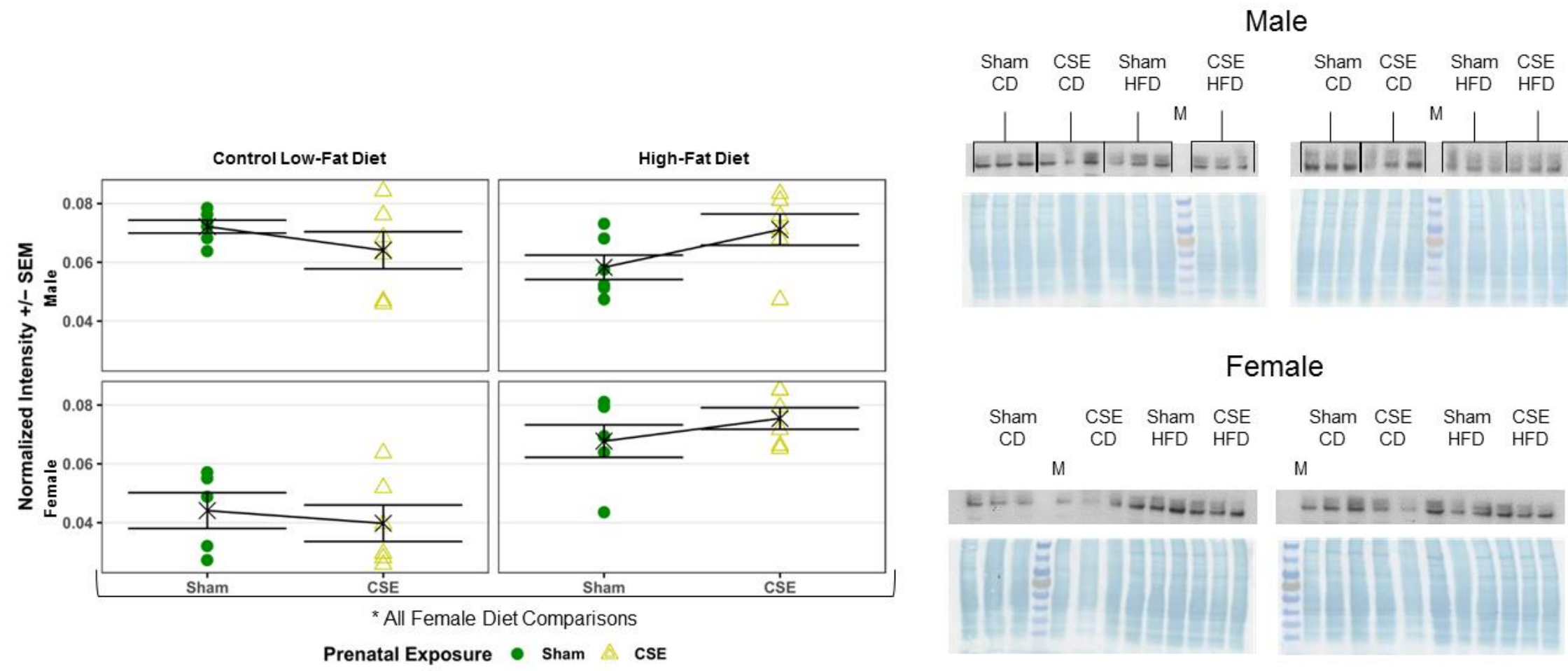

Figure 9B - Prenatal CSE does not impact hepatic NAMPT expression, while HFD increases NAMPT expression in female offspring -

Prenatal CSE had no observable effect on hepatic NAMPT levels in offspring. High-fat diet maintenance in female offspring increased hepatic NAMPT levels compared to low-fat control diet maintenance in female offspring. Hepatic NAMPT levels in male offspring were not impacted by either prenatal exposure or diet. ${ }^{*} \mathrm{p}<0.05$. 


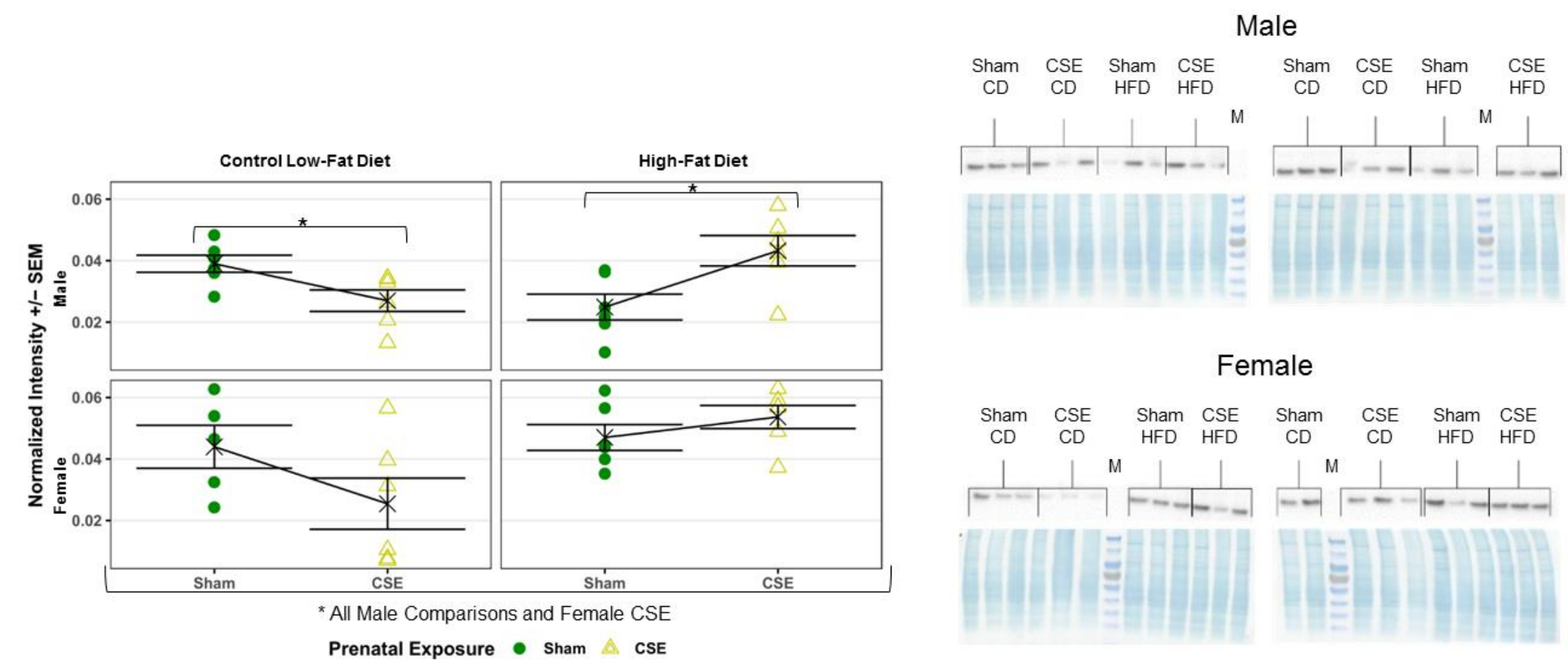

Figure 10A - The impact of prenatal CSE on hepatic GAPDH expression is highly modulated by diet in male offspring - Male CSE offspring maintained on a low-fat control diet exhibited decreased hepatic GAPDH expression compared to male Sham offspring maintained on a low-fat control diet. Male CSE offspring maintained on a high-fat diet exhibited increased hepatic GAPDH expression compared to male Sham offspring maintained on a high-fat diet. High-fat diet decreased hepatic GAPDH protein expression in male Sham offspring compared to the low-fat control diet in male Sham offspring. A high-fat diet also increased hepatic GAPDH expression in male CSE offspring compared to the low-fat control diet in male CSE offspring. A high-fat diet also increased GAPDH expression in female CSE offspring compared to low-fat control diet in female CSE offspring. ${ }^{*} p<0.05$ 
male Sham offspring decreased hepatic GAPDH expression relative to control low-fat control diet maintenance in male Sham offspring $(p<0.05)$. High-fat diet maintenance in male CSE offspring increased hepatic GAPDH protein expression relative to low-fat control diet maintenance in male CSE offspring $(p<0.05)$. Prenatal CSE had no impact on hepatic GAPHD expression in female offspring maintained on either diet. High-fat diet maintenance had no impact on hepatic GAPDH expression on female Sham offspring. High-fat diet maintenance in female CSE offspring increased hepatic GAPDH expression compared to low-fat control diet maintenance in female CSE offspring $(p<0.05)$. Our results demonstrate that in male offspring, the impact of in utero cigarette smoke exposure on hepatic GAPDH expression was conditional on diet. The expression of GAPDH in male offspring mirrored that of SIRT1 in Figure 9A except male CSE offspring maintained on a high-fat diet where SIRT1 expression was decreased while GAPDH expression was increased. These results appear to be a mismatch in expression levels in male offspring since we anticipated GAPDH levels to be in opposition to SIRT1 levels based on the typical inhibitory action of SIRT1 activity on GAPDH levels. In female offspring maintained on a high-fat diet, the increase in NAMPT expression seems inconsistent with the high levels of GAPDH since NAMPT, an enzyme whose activity is increased in a low-energy state, would seem in opposition to the promotion of glycolytic pathways.

PEPCK is a regulatory gluconeogenic enzyme that catalyzes the conversion of oxaloacetate into phosphoenolpyruvate during gluconeogenesis and is upregulated by SIRT1 activity. As seen in Figure 10B, male CSE offspring maintained on a low-fat control diet exhibited decreased hepatic PEPCK expression relative to male Sham offspring maintained on a low-fat control diet $(p<0.05)$. Prenatal CSE exhibited no impact on hepatic PEPCK expression in male offspring maintained on a high-fat diet. High-fat diet maintenance in male Sham offspring decreased hepatic PEPCK hepatic expression compared to low-fat control diet maintenance in male Sham offspring $(p<0.05)$. High-fat diet maintenance exhibited no impact on the hepatic expression of PEPCK in male CSE offspring. The hepatic expression of PEPCK in female offspring was entirely unaffected by either prenatal exposure or diet. Similar to the results seen in 

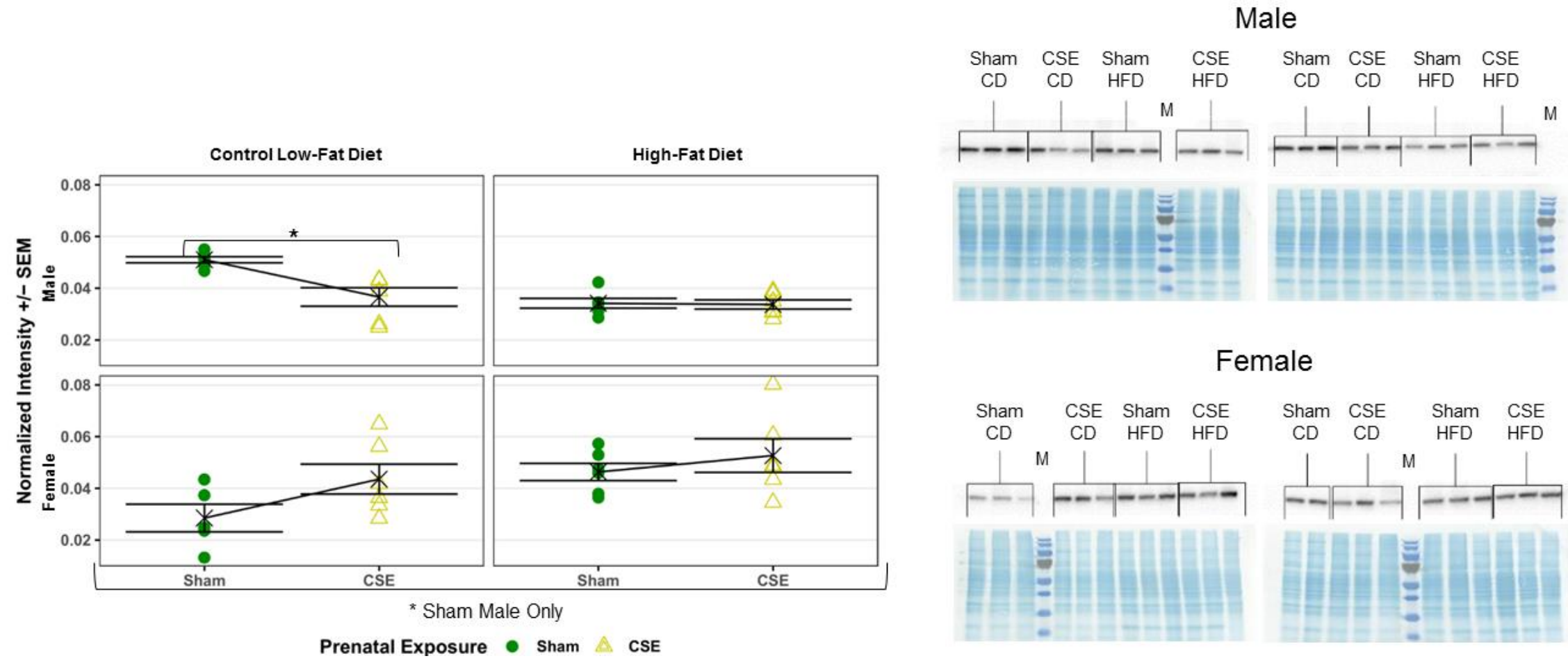

Figure 10B - Prenatal CSE and high-fat diet independently decrease PEPCK expression in male offspring - Male CSE offspring maintained on a low-fat control diet exhibited decreased hepatic PEPCK expression compared to male Sham offspring maintained on a low-fat control diet. High-fat diet maintenance in male Sham offspring exhibited decreased PEPCK expression compared to low-fat control diet maintenance in male Sham offspring. PEPCK expression was not impacted in female offspring by either prenatal exposure or diet. ${ }^{*} p<0.05$. 
Figure 9A, hepatic PEPCK expression in male offspring was decreased independently by in utero cigarette smoke exposure or maintenance on a high-fat diet. The expression of SIRT1 and PEPCK should match in general due to the stimulatory effect SIRT1 activity has gluconeogenic pathways. The results suggest that whenever SIRT1 expression was decreased in male offspring, SIRT1 activity was likely to be decreased as well since a concomitant decrease in PEPCK was observed as well. These results also mean that since SIRT1 and PEPCK were unaffected in female offspring, SIRT1 activity and gluconeogenesis pathways were relatively unimpacted.

The hepatic expression of SULT2A1, CYP8B1, SOD2, and Catalase was also measured. No impact of prenatal cigarette smoke exposure was observed for these for proteins. High-fat diet maintenance increased expression of SULT2A1 and CYP8B1 in female offspring and decreased expression of SULT2A1 in male offspring regardless of prenatal exposure (data not shown, $p<0.05)$. In the context of fatty liver disease, lipid accumulation in the liver results in the production of ROS induced by the peroxidation of lipids. One way the liver mitigates this phenomenon is by converting excess fatty acids into cholesterol and then into bile acids. In light of this, female offspring maintained on a high-fat diet on our study exhibited increased expression of both CYP8B1 and SULT2A1, two enzymes that are important in the bile acid synthetic pathway. These results may reflect the resistance of female offspring to lipid accumulation, which is in agreement with the lack of liver triglycerides in female offspring maintained on a high-fat diet.

Conversely, the decreased expression of SULT2A1 in male offspring maintained on a high-fat diet may be reflective of increased susceptibility to lipid accumulation in the liver, which is in agreement with the observed increase in liver triglycerides observed in male offspring maintained on a high-fat diet. Another process that often occurs to mitigate ROS generation from lipid peroxidation is the induction of antioxidant enzymes to neutralize ROS, such as SOD2 or Catalase. However, the lack of any impact on the expression of either of these enzymes suggests that if lipid accumulation was occurring in any of the offspring, they have not progressed to the later stages of fatty liver disease. It is evident that in light of the results observed in our 
study, none of the offspring appear to be in later stages of fatty liver disease that are typically characterized by oxidative damage to hepatic tissues.

\section{Discussion}

In our current study, the presence of a low birth weight phenotype in the murine model of gestational CSE (GD1-19) was detected with subsequent catch-up growth by the initiation of the diet challenge (six months of age). The serum cotinine levels present in the cigarette smokeexposed dams exceeded $50 \mathrm{ng} / \mathrm{mL}$, a cutoff that is indicative of active maternal smoking [305]. The low birth weight phenotype that was observed in our gestation-only exposure model is similar to that observed in other models of murine CSE [35-41]. Unlike models of both pre- and postnatal exposure to cigarette smoke (GD6-PD21) in which offspring exhibited low birth weight and had not caught up by six months of age [35-40], the exposed offspring in our gestation-only CSE model did exhibit catch-up growth by six months of age in both male and female offspring. Other studies using a gestation-only model of cigarette smoke exposure found a decrease in body weight of $10-12 \%$ that persisted 14 [313] and 30 days [314] after birth. The model showing a persistent weight decrement at 14 days after birth was exposed only from E8-E20, with urine cotinine levels in the dams of $5,800 \mathrm{ng} / \mathrm{mL}$ [313]. The study showing decrements at 30 days utilized a nose cone-based inhalation model where the mothers were exposed for three weeks before breeding [314] that could have impacted neonatal growth. That same study could have confounding introduced by the animal restraint that needs to be used to conduct a nosecone inhalation exposure. According to their methodology, before breeding, females were subjected to two exposure sessions of varying duration per day [314]. Additionally, they did not define duration in terms of time, but in the number of puffs per session (24 puffs per session throughout gestation) [314]. A study examining the effects of neonatal thirdhand cigarette smoke exposure in C57BL/6 mice found that offspring housed with terrycloth exposed to $5600 \mathrm{mg}$ of cigarette smoke particulate material from birth to three weeks after birth had decreased weight at 3-weeks old and complete catch-up growth at 5-weeks old [315]. It appears that the overall duration, intensity, timing, and maternal or paternal-only exposure to cigarette smoke exposure 
may play a role in whether catch-up growth occurs in affected offspring. It is evident that other models of pre- and periconception CSE also found low birth weight with persistent decrements in weight. The current study, which monitored weights for a considerably greater length of time, found catch-up growth. It should be noted that these studies vary in type of cigarette smoke exposure (nose cone or whole body) and other measures of duration and quantity of cigarette smoke exposure. The current model of prenatal cigarette smoke exposure does fit the Barker Hypothesis with prototypical low birth weight followed by subsequent catch-up growth in exposed offspring.

It was clear from the data presented from our current study that in utero cigarette smoke exposure exacerbates sex-specific weight gain in sexually mature mice with modulation by postnatal diet. This finding is similar to a study by $\mathrm{Ng}$ et al. that found that $13-$ to 14 -week old mouse female offspring exposed in utero to cigarette smoke and maintained on a control diet were heavier than air-exposed offspring [46]. They also found that female CSE offspring, when maintained on a high-fat diet for two weeks, were not different from air-exposed female offspring [46]. That same study found male offspring exposed in utero to cigarette smoke and maintained on a high-fat diet weighed more than air-exposed male offspring, with no impact of prenatal CSE when male offspring were fed a control diet [46]. However, in our study it was the female offspring exposed to prenatal cigarette smoke and high-fat diet maintenance that exhibited elevated body weight gain with no impact of prenatal CSE when maintained on a control diet. There are several methodological differences between our study and the $\mathrm{Ng}$ et al. study that complicates the comparison of outcomes. For example, the cigarette smoke produced in the $\mathrm{Ng}$ et al. study resulted in a lower CO level (23.4 ppm vs. 110.88 ppm), a lower total suspended particulate level (17.1 $\mathrm{mg} / \mathrm{m}^{3}$ vs $\left.29.14 \mathrm{mg} / \mathrm{m}^{3}\right)$, used a different strain of mice $\left(\mathrm{B}_{6} \mathrm{C}_{3} \mathrm{~F}_{1}\right.$ vs $\left.\mathrm{C} 57 \mathrm{BL} / 6\right)$, different start of gestational exposure ( 4 days after mating vs 6 days after mating), different timing and duration of diet challenge (began at 11-12 weeks and lasted for 2 weeks vs began at 6 months and lasted for 3 months), and different fat percentage content of the high-fat diet fed to offspring ( $21.2 \%$ fat versus $19.9 \%$ fat) [46]. Despite methodological differences, it is evident that in our study adult female offspring subjected to in utero cigarette smoke exposure and maintained on a high-fat diet are more 
impacted than were the male offspring. Overall, we found that the impact of in utero cigarette smoke exposure in sexually mature mice results in differential weight gain that is modulated by both the postnatal diet and sex of the offspring.

In the current study, prenatal cigarette smoke exposure had a limited impact on the total quantity of calories consumed during the diet challenge. Maintenance of offspring on a high-fat diet increased the total amount of calories consumed by offspring regardless of prenatal exposure. As far as we know, there has not been a published study before ours that observed calorie intake of adult offspring maintained on various diets in an animal model of prenatal CSE. One study has found that children exposed in utero to cigarette smoke had higher fat consumption and total body fat [316]. A cross-sectional study found that adolescent children subjected to in utero cigarette smoke exposure consume more fat through epigenetic modification of Oprm1 [317]. In the current study, we were not able to recapitulate the effects of prenatal cigarette smoke exposure on calorie consumption found in the literature. However, the fact that calorie consumption was tracked on a per-cage basis rather than on an individual basis weakens the interpretation of our findings. Future studies should track individual calorie consumption in order to fully determine the role of prenatal cigarette smoke exposure on eating habits during adulthood.

The current study documents that female offspring exposed in utero to cigarette smoke did display less anxiety behavior, as demonstrated by more time spent in the central region in an open field assessment. We did not characterize differences in offspring anxiety in CSE offspring maintained on a low-fat control diet. Unlike our study, a prospective cohort study found that prenatal exposure to cigarette smoke was associated with children age two to three years exhibiting more anxiety behavior, particularly in girls [318]. Similar to our study, a study utilizing both preand postnatal cigarette smoke exposure in mice found that exposed female offspring were less anxious [44]. Similar to our study, rats exposed in utero to $5 \mathrm{mg} / \mathrm{kg} / \mathrm{day}$ to nicotine through an implanted minipump from gestational day 8-20 exhibited less anxiety behavior at 12-14 months of age [319]. It is evident that prenatal CSE or nicotine influences anxiety behavior; however, the directionality and which sex is more affected appears to vary. Even though our results are not 
entirely consistent with the literature, it is clear that in utero cigarette smoke exposure impacts neurobehavioral development.

The current study also found that female offspring exposed in utero to cigarette smoke and maintained on a high-fat diet were more active than the corresponding male offspring both in terms of average speed traveled, and the total distance traveled. Similar to the outcomes in our study, a prospective cohort study found that children subjected to prenatal cigarette smoke exposure were more hyperactive [320]. Unlike our study, however, the male offspring were more affected and had more attention and aggression behavioral problems [320]. A previously mentioned pre- and postnatal exposure found prenatal CSE offspring were more active anxious [44], much like the female CSE offspring from our current study. A study found male $\mathrm{B}_{6} \mathrm{C}_{3} \mathrm{~F}_{1}$ mice exposed in utero to cigarette smoke from GD4 to parturition exhibited increased locomotor activity at four weeks after birth and 4-6 months of age, similar to our current findings, and was found to co-occur with increased aggression [321]. A mouse model using C57BL/6 mice found that prenatal exposure to nicotine resulted in increased male offspring locomotor activity [322], unlike our study, where it was female offspring that displayed increased locomotor activity. The only notable difference seen concerning in utero cigarette smoke exposure was the decreased anxious behavior seen in female CSE offspring when maintained on a high-fat diet. We did not characterize differences in offspring activity in CSE offspring maintained on a low-fat control diet. It did not appear from our results that activity mediated the impact of in utero cigarette smoke exposure on exacerbated weight gain in female offspring with high-fat diet maintenance. Despite the increased levels of activity seen in female CSE offspring compared to male CSE offspring, female CSE offspring still experienced a significant increase in weight gain when fed a high-fat diet while male CSE offspring only exhibited a trend toward increased weight gain when maintained on a high-fat diet. This exemplifies just how profoundly the metabolism of CSE female offspring is adversely affected with respect to resisting high-fat diet induced weight gain.

The current study demonstrates that male offspring exposed to in utero cigarette smoke exhibited differences in hepatic protein expression of several key metabolic enzymes involved with glucose metabolism, including decreased SIRT1, GAPDH, and PEPCK expression whereas livers 
of female offspring were unaffected. The literature has examples of decreased SIRT1 and NAMPT expression in the liver of mice fed a high-fat diet [261, 262]; however, in the current study, high-fat diet consumption resulted in no impact on hepatic NAMPT expression in male offspring and increased hepatic NAMPT expression in female offspring. The current study did replicate in the male offspring the decrease in hepatic SIRT1 expression with high-fat diet feeding that is seen in the literature [261, 262], but not for female offspring maintained on a high-fat diet. In a murine model of pre- and postnatal cigarette smoke exposure, decreased hepatic SIRT1 expression in 6month old offspring was observed but increased PEPCK expression [40]; however, that study used mice in a fed state whereas the current study the offspring were in a fasted state before euthanization. Fasting state is essential to consider when interpreting these results since a typical fasting response includes increased SIRT1 expression and activity along with increased gluconeogenic activity via increased expression and activity of PEPCK and GAPDH in the gluconeogenesis direction.

A remaining question about SIRT1 and Sirt1 expression is whether the change is due to the intrauterine growth restriction or directly by the cigarette smoke exposure. One study found in C57BL/6 mice given twice daily nicotine plus fed a high-fat diet for ten weeks had both reduced hepatic SIRT1 protein and RNA expression [323]. Male A/J mice injected with nicotine three times a week for ten weeks after an initial injection with NNK 2 weeks before that exhibited decreased SIRT1 expression in the lung [324]. A more recent study found that $\mathrm{Apo}^{-/-}$mice subjected to ecigarette exposure containing $2.4 \%$ nicotine for 12 weeks exhibited decreased hepatic SIRT1 expression along with a decreased ratio of $\mathrm{NAD}^{+} / \mathrm{NADH}$ and increased hepatic PARP1 (a known consumer of $\mathrm{NAD}^{+}$) expression [325]. Although these studies were not prenatal exposures like in our studies, they offer some evidence that cigarette smoke may be able to influence SIRT1 levels directly rather than indirectly by causing intrauterine growth restriction. Given the evidence, it seems probable that the hepatic SIRT1 decrease in expression seen in the male offspring in our current study could be due to direct effects of cigarette smoke on hepatic tissue that remain all the way into adulthood, however, more studies need to be done to determine whether this is a direct action of cigarette smoke or indirectly via intrauterine growth restriction. 
There were several limitations to the current study that prevented extracting as much information as we could have. For example, tracking individual offspring weight from birth until the end of the study was not conducted. This tracking would have allowed the determination of associations between magnitudes of low birth weight and subsequent expression of hepatic proteins and phenotypic characteristics at adulthood. In future studies, we plan to take measures to allow weight tracking across the entire lifetime of offspring to strengthen the outcomes observed. Another limitation was not observing the expression of SIRT1 and other hepatic proteins in sibling animals at six months of age, before the initiation of the diet challenge. Taking note of this would have provided information on the expression of SIRT1 on a diet that was intermediate in fat content $(\sim 12 \%)$ and any change in expression after three months of maintenance on a low-fat control diet ( $5 \%$ fat $)$ or a high-fat diet ( $20 \%$ fat). Taking note of this would also be essential to note since this design would have allowed us to investigate how prenatal CSE impacted modulation of SIRT1 on these diets. In a currently ongoing experiment, we have modified our design presented here to record differences in hepatic protein expression at a point before induction of a diet challenge in order to address this issue. Using a low-fat diet as a control for the diet challenge may have complicated the interpretation of our results by modulating the total calories consumed. The choice of control diet is an essential distinction since, as mentioned earlier in this report, SIRT1 is a sensor of the current cellular energy status where its activity is classically upregulated in a low-energy status. As a result, our choice of a low-fat diet as the control of the high-fat diet potentially exacerbated any differences in SIRT1 expression and the expression of related glucose metabolic enzymes. In the future, we plan to modify our experimental design to use a more appropriate control for high-fat diet maintenance. Despite these limitations, we think the data presented here provide new insight into the sex-specific impacts on the liver in a model of in utero cigarette smoke exposure and how this is modulated by adulthood diet.

Our model of prenatal cigarette smoke exposure (from gestational day 6-19) resulted in sexually dimorphic weight gain and hepatic SIRT1 protein and enzyme expression associated with glucose metabolism. The low birth weight predicted by the Barker Hypothesis was apparent. The resistance to perturbation by prenatal cigarette smoke exposure observed in female offspring is 
potentially due in part to increased levels of estrogen signaling in females. We hypothesize that females may be more resistant to developing non-alcoholic fatty liver disease in part due to being able to resist perturbation of hepatic SIRT1 expression and subsequent modulation of glucose metabolic pathways. Future studies could confirm this by examining levels of estrogen signaling activation in offspring exposed in utero to cigarette smoke and search for correlations with the phenotypes demonstrated in the current study. Future studies should examine whether the effects on hepatic protein expression observed here also are present at earlier time points in the lifecycle of in utero cigarette smoke-exposed offspring such as at weaning or during the perinatal period. The possibility that the perturbations induced by prenatal cigarette smoke exposure occurs regardless of the current energy status of the animal or specifically disrupts the fasting response to a low-energy state is the focus of subsequent chapters. 


\section{CHAPTER III: IN UTERO CIGARETTE SMOKE EXPOSURE PERTURBS HEPATIC EXPRESSION OF METABOLIC PATHWAYS IN A SES-DEPENDENT MANNER SHORTLY AFTER BIRTH}

\section{Introduction}

The reported rate of smoking during pregnancy in the U.S. has fallen over the years. The earliest recorded data from 1967 and 1980 National Natality Surveys show the rate of smoking while pregnant in women age 20 years or older dropped from $40 \%$ to $25 \%$ in Caucasian women and from $33 \%$ to $23 \%$ in African American women [326]. It has continued to fall since, with the overall rate of smoking during pregnancy in the U.S. being 18.4\% [50], 12.2\% [327], and 10.7\% [328] in 1990, 2000, and 2010, respectively. According to the latest data available in the United States, smoking during pregnancy has fallen from a rate of $8.4 \%$ to $7.2 \%$ from 2014 to 2016 [47, 48]. An initial examination makes it seem as though public health campaigns to inform the public about the adverse effects of in utero cigarette smoke exposure to the developing fetus have been successful. Examining the data from Kentucky and West Virginia however reveals maternal smoking during pregnancy occurs at a rate of $18.4 \%$ and $25.1 \%$, respectively [47]. It is evident that although overall the rate of smoking during pregnancy has gone down dramatically, there are still states that have disproportionately higher rates than others.

A point of concern is that most of this data is highly dependent on self-reported smoking during pregnancy. For example, a study utilizing NHANES data from 1999-2006 found that $22.9 \%$ of pregnant women fail to disclose their current smoking status, which was confirmed by serum cotinine levels that exceeded a defined cut-off for actively smoking [51]. Underreporting of smoking during pregnancy was also evident in a study comparing 2003 data from the Pregnancy Risk Assessment Monitoring System (PRAMS) and from states that had implemented the revised 2003 standard birth certificate. In that study, it was shown that PRAMS found that $14.0 \%$ of women smoked during pregnancy while data from the revised 2003 standard birth certificate found that 
$11.3 \%$ of women smoked during pregnancy [329]. Another example is a study utilizing data from New York City and Vermont, where the rate of smoking during pregnancy was $10.4 \%$ using the 2003 U.S. Standard Birth Certificate and 14.1\% using medical records [330]. Utilizing data that relies on self-reported maternal smoking habits potentially results in significantly underreporting the rates of smoking during pregnancy. It is hypothesized that a reason for this phenomenon of underreporting is due to more well-educated women being concerned about being judged by their peers more harshly than less-educated women are [48]. For example, the study utilizing data from New York City and Vermont found a significantly higher discrepancy between birth certificate and medical records; the more educated the mother was and if they had private insurance vs Medicaid coverage [330]. These studies make evident that if proper care is not taken to collect data accurately, we risk significantly underestimating the rate of smoking during pregnancy, and by extension, the number of infants exposed in utero to cigarette smoke.

A significant primary outcome of cigarette smoke exposure (CSE) during gestation in infants is intrauterine growth retardation, which presents as low birth weight [28, 29, 138-142]. Models of mice [35-41], as well as rats [43], also exhibit this phenotype. As described in the Introduction of Chapter II, the Barker Hypothesis predicts increased metabolic disease during adulthood as a result of prenatal exposures such as maternal caloric restriction. As mentioned in Chapter II, the similarities between prenatal cigarette smoke/nicotine exposure and maternal caloric restriction result in a common manifestation of low birth weight and catch-up growth phenotypes. These similarities in phenotypes between prenatal cigarette smoke exposure and maternal caloric extension, by extension results in similar risks for metabolic disease in adulthood. Although the impact of prenatal cigarette smoke exposure has been documented in epidemiological studies, not much has been done to examine precisely how the metabolism of offspring exposed in utero to cigarette smoke on a mechanistic level. In studies previously conducted by our lab with a mixed-sex murine model using pre- and postnatal exposure to cigarette smoke, it was found that exposed offspring had a perturbed metabolism [35, 331]. Our lab showed that exposed offspring at PD21 experience decrements in glucose, lipid, and amino acid metabolic proteins according to proteomic analysis [35]. Specifically, in a fed state, 6-month-old exposed offspring had decreased 
levels of hepatic SIRT1 protein as well as increased levels of PEPCK and PGC1 $\alpha$ protein and decreased serum glucose levels [331]. Exposed offspring also exhibited oxidative stress in the form of depleted glutathione [331]. One crucial facet of the Barker hypothesis that is not addressed by these studies is the examination of the sex-specific impact of prenatal CSE on liver metabolism. Additionally, these studies do not address whether liver metabolic changes can be detected in the perinatal period and whether these changes persist into adulthood. Determining this would allow for the possible development of targeted early-life interventions in cases of prenatal exposure to cigarette smoke.

As mentioned previously in Chapter II, SIRT1 is a NAD+-dependent enzyme that deacetylates acetyllysine residues on proteins and is induced through the sensing of low energy status through levels of $\mathrm{NAD}^{+}$[255]. As an essential sensor of low-energy status, SIRT1 deacetylates key transcription factors in order to promote the hepatic fasting response, including induction of gluconeogenic and lipolytic processes while inhibiting glycolytic and lipogenic processes [255]. After deacetylating transcription factors responsible for the regulation of these metabolic processes, these modified transcription factors result in the induction or inhibition of key metabolic enzymes. For example, SIRT1-mediated deacetylation of FOXO1 [332] and PGC-1 $\alpha$ [333] leads to enhanced transcription of selected gluconeogenic enzymes such as PEPCK. Seemingly paradoxically, SIRT1 deacetylation of the gluconeogenic transcription factor CRTC2 promotes its degradation [258]; however, this is thought to occur in order to sustain gluconeogenesis at a steady rate during a prolonged fasting response [334]. Through deacetylation, SIRT1 also enhances the activity of PPARa [335, 336], a critical transcription factor for promoting transcription of lipolytic enzymes such as EHHADH and MCAD. Further, in support of the fasting response, SIRT1 deacetylates the lipogenic transcription factor SREBP-1C [337], which results in lower transcription of lipogenic enzymes such as FAS in order to avoid conflicting with lipolytic processes. There are additional metabolic processes that SIRT1 influences, such as the bile acid sensor and transcription factor FXR, where deacetylation promotes protein stability, thereby enhancing its transactivation function on downstream targets such as SHP [256], an essential negative regulator of CYP7A1 expression. 
The objective of this study was to characterize the sex-specific impact of prenatal CSE on hepatic SIRT1 expression on target gene expression during the neonatal period, at a time at which low birth weight is no longer evident (1.5 weeks of age). We utilized an established mouse inhalation model of cigarette smoke exposure that mimics active maternal smoking during pregnancy throughout the entire fetal development period (GD1-GD19). The mRNA gene expression levels for SIRT1 targets, which are indicative of activation or suppression, are indirect indicators of SIRT1 activity. A similar in utero cigarette smoke exposure paradigm leads to sexspecific modulation of fat deposition, weight gain, liver SIRT1, GAPDH, and PEPCK protein expression, as seen in Chapter II.

\section{Methods}

\section{Animal Exposure}

Female C57BL/6 mice maintained on breeder chow (LabDiet 5015) were mated overnight and checked the following day for the presence of vaginal sperm. Sperm positive status was considered gestational day (GD) 0 for the exposure timing. On GD1, female C57BL/6 mice were placed into either a cigarette smoke exposure (CSE) chamber or a sham exposure (ambient filtered room air) chamber for 6 hours/day daily from GD1 to GD19. Cigarette smoke exposure was carried out using a Teague TE-10C exposure apparatus (Teague Industries) smoking Marlboro Red cigarettes, which was the most commonly smoked cigarette at the time of model development, using the FTC method of smoking (2-second puff per minute). After parturition, dams with litters were maintained on breeder chow. At 1.5 weeks of age, representative offspring of each litter were euthanized by carbon dioxide asphyxiation. At euthanization, the excised liver was frozen on dry ice and stored at $-80^{\circ} \mathrm{C}$.

\section{Cigarette Smoke Exposure Monitoring via Measurement of Carbon Monoxide, Total Suspended Particulates, and Plasma Cotinine}

At the start of each day of exposure, the level of $\mathrm{CO}$ within the cigarette smoke exposure chamber was allowed to increase rapidly by fully closing the dilutor valve until the level reached 
110 parts per million (ppm) as detected with a commercially available carbon monoxide detector with a digital readout. Once this level was reached, $\mathrm{CO}$ levels were further adjusted by opening and closing the dilutor valve as appropriate to maintain a target CO level ranging between 110 to $150 \mathrm{ppm}$. At the end of exposure each day, the dilutor valve was fully opened to allow rapid dissipation of any remaining $\mathrm{CO}$ in the cigarette smoke exposure chamber. TSPs were recorded twice daily by drawing air from each exposure chamber via a sampling port through a preweighed piece of filter paper. The filter paper was then reweighed, and the pre- and the post-sampling difference was divided by the volume of air drawn through the sampling device in five minutes. Levels of CO were also recorded at the time of TSP recordings. In order to quantitate the magnitude of cigarette smoke exposure, cotinine, the primary metabolite of nicotine, was measured in representative females immediately after cessation of the exposure period on days 14-18. Tail vein blood was collected from dams within 30 minutes of the end of exposure into heparinized capillary tubes and was subsequently centrifuged at $10,000 \times g$ for 10 minutes. The collected plasma fractions were stored at $-20^{\circ} \mathrm{C}$ until ready for cotinine measurement. Using the Cotinine One-Step ELISA Detection Kit (International Diagnostic Systems; St. Joseph, MI), the collected plasma fractions were assayed for the concentration of cotinine following the manufacturer's instructions. The absorbance of the reaction substrate was measured at $450 \mathrm{~nm}$ using a SpectraMax 190 Microplate Reader (Molecular Devices, San Jose, CA) and reported as nanograms per milliliter. Levels of plasma cotinine greater than or equal to $50 \mathrm{ng} / \mathrm{mL}$ were considered sufficient to simulate active maternal smoking during pregnancy.

\section{Offspring Health and Serum Glucose Measurement}

Animals were housed and maintained at the University of Louisville Research Resources Center, an Association for Assessment and Accreditation of Laboratory Animal Care accredited facility. All protocols were approved by the UofL Institutional Animal Care and Use Committee. Cages for animal housing were changed weekly, unlimited food and water provided, and maintained in an environment with a 12-hour light/dark cycle with controlled temperature and humidity. Offspring were weighed daily and checked for health indicators such as levels of 
activity and eye-opening. Blood was collected from offspring after euthanization via cardiac puncture using heparinized syringes. Blood samples were subsequently centrifuged at $10,000 x$ $g$ for 10 minutes, and the separated plasma fraction was collected and stored at $-80^{\circ} \mathrm{C}$.

Using the Glucose (HK) Assay Kit (Catalog \# GAHK20; Sigma-Aldrich; St. Louis MO), the collected plasma fractions were diluted 50 -fold and were assayed for the concentration of glucose following the manufacturer's instructions. The absorbance of the reaction substrate was measured at $340 \mathrm{~nm}$ using a SpectraMax 190 Microplate Reader (Molecular Devices, San Jose, $\mathrm{CA}$ ) and reported as $\mathrm{mg}$ glucose $/ \mathrm{mL}$.

\section{Western Blotting}

Liver was homogenized in ice-cold buffer [100 mM PO4 Buffer, $150 \mathrm{NaCl}, 0.1 \%$ Tween, $0.1 \%$ SDS, $0.5 \mathrm{M}$ Tris $\mathrm{pH} 8.0,2$ tabs per $10 \mathrm{~mL}$ phosphatase and protease inhibitor cocktails, 10 $\mu \mathrm{L}$ per $10 \mathrm{~mL}$ EDTA, $7.5 \mathrm{mg} / \mathrm{mL}$ dithiothreitol (DTT)] using a Tissue-Tearor homogenizer in 2second on/off pulses over the span of 1 minute. The concentration of protein was measured by the Bradford method [301]. Liver homogenates were mixed with Laemmli buffer (0.25M Tris $\mathrm{pH}$ 6.8, glycerol, $10 \%$ SDS, trace bromophenol blue), reduced with DTT $(1 \mathrm{~g} / 5 \mathrm{~mL})$ then heated at $70^{\circ}$ C for 10 minutes. Thirty-five $\mu \mathrm{g}$ total protein was applied to $10 \%$ acrylamide gels with separation performed at $100 \mathrm{~V}$ for 2 hours in Tris-glycine buffer ( $25 \mathrm{mM}$ Tris, $192 \mathrm{mM}$ glycine, $\mathrm{pH}$ 8.3) followed by transfer to PVDF membrane at $70 \mathrm{~V}$ for 2 hours in a non-reducing buffer [Tris-Glycine buffer (25 mM Tris, $192 \mathrm{mM}$ glycine, pH 8.3) with 20\% ethanol]. Following blocking in 5\% non-fat dry milk in TBS-T solution (Tris-Buffered Saline-Tween, $137 \mathrm{mM} \mathrm{NaCl}, 20 \mathrm{mM}$ Tris-Base pH 7.6, $0.1 \%$ Tween-20), the blots were incubated overnight at $4^{\circ} \mathrm{C}$ with primary antibody (SIRT1 $1 / 500$, Santa Cruz \#sc-15404; PEPCK 1/500, Santa Cruz \#sc-271029; HK 1/500, Cell Signaling \#2024; NAMPT 1/1000, Thermo-Fisher \#PA5-23198; ac-lysine 1/250, Millipore \#AB3879; ac-FKHR 1/250, Santa Cruz \#sc-49437; FKHR 1/500, Santa Cruz \#sc-11350) diluted in non-fat dry milk in TBS-T. After three washes of 10 minutes each in TBS-T, blots were incubated with secondary antibody complexed to horseradish peroxidase (anti-mouse, Thermo \#32430; anti-rabbit, Cell Signaling \#7074S) in non-fat dry milk diluted in TBS-T at room temperature for 3 hours. After 
three successive washes in TBS-T, blots were developed with LumiGLO chemiluminescent reagent and peroxide (Cell Signaling Technology \#7003S, Danvers, MA), and visualized with a Bio-Rad Gel Doc XR+ system (Bio-Rad Laboratories, Hercules, CA). Following visualization, blots were washed briefly in TBS-T and incubated with Coomassie stain $(0.1 \%$ Coomassie Brilliant Blue G250 Dye in 1:1 methanol:water) for 10 minutes, followed by two 10 minute washes in destaining solution (5:4:1 ethanol:water:acetic acid), and a final wash for 5 minutes in deionized water. Blots were briefly air-dried and scanned for quantitation of total protein.

\section{Real-Time Quantitative PCR (RT-qPCR)}

At euthanization, a section of the liver was equilibrated overnight in RNAlater at $4^{\circ} \mathrm{C}$, after which it was placed into long term storage at $-80^{\circ} \mathrm{C}$. A portion of RNAlater-stabilized liver was used for RNA extraction using the Qiagen RNeasy Mini Kit (Qiagen, Germantown, MD) per manufacturer's instructions. The final elution was carried out using two aliquots of nuclease-free water and was stored at $-80^{\circ} \mathrm{C}$. RNA concentration was determined using a NanoDrop 8000 Spectrophotometer (Thermo Scientific, Waltham, MA). Using two $\mu \mathrm{g}$ of each isolated RNA sample as input, cDNA was synthesized using the High-Capacity cDNA Reverse Transcription Kit following the manufacturer instructions (Applied Biosystems cat\# 4368813). cDNA reactions were carried out as $20 \mu \mathrm{L}$ reactions in PCR tubes placed in a Bio-Rad MyCycler (Bio-Rad Laboratories, Hercules, CA). The cDNA reactions were set for an initial 10 minutes at $25^{\circ} \mathrm{C}$, followed by 2 hours at $37^{\circ} \mathrm{C}$ and 5 minutes at $85^{\circ} \mathrm{C}$. The cDNA reaction tubes were held at $4^{\circ} \mathrm{C}$ until placed into storage at $-20^{\circ} \mathrm{C}$.

Mouse 18S rRNA gene was used as the reference gene for RT-qPCR. The genes of interest have the forward and reverse primer sequences listed in Table 1. PCR reactions were carried out as $10 \mu \mathrm{L}$ reactions in 96 well plates and ran using a Bio-Rad iQ5 Multicolor Real-Time PCR Detection System (Bio-Rad Laboratories, Hercules, CA) in 96 well plates. The PCR reactions were set for an initial $3 \mathrm{~min}$ at $95^{\circ} \mathrm{C}$, followed by 40 cycles of $15 \mathrm{sec}$ at $95^{\circ} \mathrm{C}$ and $1 \mathrm{~min}$ at $60^{\circ} \mathrm{C}$ using SsoAdvanced Universal SYBR Green Supermix (Bio-Rad Laboratories, Hercules, CA). Each PCR plate was filled out with three technical replicates per sample and three 


\begin{tabular}{|c|c|c|c|}
\hline$\#$ & Name & Forward Primer & Reverse Primer \\
\hline 1 & SIRT1 & GCAGGTTGCGGGAATCCAA & GGCAAGATGCTGTTGCAAA \\
\hline 2 & NAMPT & CATAGGGGCATCTGCTCATT & GCTGCTGGAACAGAATAGCC \\
\hline 3 & PGC-1A & ATGTGTCGCCTTCTTGCTCT & АTCTACTGCCTGGGGACCTT \\
\hline 4 & CRTC2 & GCCACATTGACAGTTCTCCA & AAGTGTCTTGGGGGTTAGGG \\
\hline 5 & SREBP-1C & ATGGATTGCACATTTGAAGACATGCTC & CCTGGGCTGCTGGGGCCTG \\
\hline 6 & PPARa & AGAAGTTGCAGGAGGGGATT & TCGGACTCGGTCTTCTTGAT \\
\hline 7 & FGF21 & CAAATCCTGGGTGTCAAAGC & ATTGTAACCGTCCTCCAGCA \\
\hline 8 & FGFR4 & GACCAAACCAGCACCGTGGCTGTGAAGATG & GTTTCCCTTGGCGGCACATTCCACAATCAC \\
\hline 9 & B-Klotho & CGAGCCCATTGTTACCTTGT & CTCCAAAGGTCTGGAAGCAG \\
\hline 10 & CYP7A1 & CAGGGAGATGCTCTGTGTTCA & AGGCATACATGCAAAACCTCC \\
\hline 11 & CYP8B1 & TTCGACTTCAAGCTGGTCGA & CAAAGCCCCAGCGCCT \\
\hline 12 & SHP & AAGGGCACGATCCTCTTCAA & CTGTTGCAGGTGTGCGATGT \\
\hline 13 & BSEP & CTGCCAAGGATGCTAATGCA & CGATGGCTACCCTTTGCTTC \\
\hline 14 & OSTB & GATGCGGCTCCTTGGAATTA & GGAGGAACATGCTTGTCATGAC \\
\hline 15 & GAPDH & AAATGGTGAAGGTCGGTGTGAAC & CAACAATCTCCACTTTGCCACTG \\
\hline 16 & PEPCK & TATGCTGATCCTGGGCATAA & CACGTTGGTGAAGATGGTGT \\
\hline 17 & G6Pase & TCTGTCCCGGATCTACCTTG & GTAGAATCCAAGCGCGAAAC \\
\hline 18 & HK & TATGAAGACCGCCAATGTGA & TTTCCGCCAATGATCTTTC \\
\hline 19 & PKM2 & TCGCATGCAGCACCTGATT & CCTCGAATAGCTGCAAGTGGTA \\
\hline 20 & EHHADH & CCGGTCAATGCCATCAGT & CTAACCGTATGGTCCAAACTAGC \\
\hline 21 & FAS & GCTGCGGAAACTTCAGGAAAT & AGAGACGTGTCACTCCTGGACTT \\
\hline 22 & MCAD & GGTTTGGCTTITGGACAATG & TGACGTGTCCAATCTACCACA \\
\hline 23 & FXR & TCCACAACCAAGTTTTGCAG & TCTCTGTITGTTGTACGAATCCA \\
\hline 24 & LXR-a & TGAGAGCATCACCTTCCTCA & TGGAGAACTCAAAGATGGGG \\
\hline 25 & LRH1 & AACGATGTCCCTACTGTCGATT & CATGCGGTCGGCTCTTAC \\
\hline 26 & HNF4A & CAGCAATGGACAGATGTGTGA & TGGTGATGGCTGTGGAGTC \\
\hline 27 & IL-6 & CTGCAAGAGACTTCCATCCAG & AGTGGTATAGACAGGTCTGTTGG \\
\hline 28 & MCP-1 & CAGGTCCCTGTCATGCTTCT & GAGGATCACCAGCAGCAGGT \\
\hline 29 & TNF-a & AAGCCTGTAGCCCACGTCGTA & AGGTACAACCCATCGGCTGG \\
\hline 30 & COL1A1 & TAGGCCATTGTGTATGCAGC & ACATGTTCAGCTTTGTGGACC \\
\hline 31 & PDK-4 & GCCTTGGGAGAAATGTGTGT & CACTGGCTITITGAGTGCAA \\
\hline 32 & P53 & GTCACAGCACATGACGGAGG & TCTTCCAGATGCTCGGGATAC \\
\hline 33 & $18 \mathrm{~S}$ & CTCAACACGGGAAACCTCAC & CGCTCCACCAACTAAGAACG \\
\hline
\end{tabular}

Table 1: Forward and reverse primer pairs for RT-qPCR 
template-free controls per primer pair. Data were analyzed using the $\Delta \Delta \mathrm{CT}$ method followed by $\log 2$ transformation of fold-change values.

\section{Imputation of Missing Weight Data}

Offspring weights were recorded from PD1 (the day after birth) until weaning (PD19).

Occasionally, throughout the neonatal monitoring period, recording of daily weighs was missed. In order to utilized linear mixed-effects modeling, imputation was performed. Imputation was accomplished using the mice $\mathrm{R}$ package [338] by the predictive mean matching method utilizing a value of $\mathrm{m}$, the number of datasets to impute, of 50 with 100 max iterations. At the end of the imputation procedure, all 50 potential imputed values generated for each missing value were averaged together and merged back with the original dataset to render it complete. This completed dataset was then used for offspring weight analyses.

\section{Linear Mixed-Effects Modeling}

Linear mixed-effects modeling using the Ime4 R package [302] was conducted to assess the effect of prenatal CSE on hepatic protein and mRNA expression levels. The general mixedeffects equation utilized to model the data is given by:

Eq. 1: Variable of Interest $\sim 1+$ Exposure $+(1 \mid$ Cohort $)$

Where Exposure indicates modeling of fixed effects for prenatal exposure. The (1/Cohort) term indicates the identity of the cohort set as a random intercept. The random intercept was set this way to account for the fact that each individual offspring is more likely to be similar to other littermates than offspring from different litters. The goal of this was to help control potential confounding of results due to potential natural variance between litters. In instances where all individuals within each comparison to be done were all from the same cohort, the following equation was used instead: 
Eq. 2: Variable of Interest $\sim 1+$ Exposure

The above equation 2 is similar to 1 but without the (1|Cohort) random intercept term. For some analyses, fitting random-effects models were not possible due to the occurrence of singular fits. For the models using Eq. 1 listed above that resulted in a singular fit, Eq. 2 was instead used. Post-hoc testing was conducted using t-tests for specific a priori contrasts of interest using the finalized models with the emmeans R package [303]. The contrast of interest for all RT-qPCR experiments was CSE versus Sham. For analysis of RT-qPCR data, log2 transformed foldchange values were used while Western blot analysis used protein expression values normalized by total protein.

\section{$\underline{\text { Results }}$}

\section{Cigarette Smoke Exposure Parameters and Low-Birth Weight Phenotype}

The average carbon monoxide level in the cigarette smoke exposure chamber was 147 ppm $+/-43$ ppm, while total suspended particulates averaged $26.8 \mathrm{mg} / \mathrm{m}^{3}+/-7.5 \mathrm{mg} / \mathrm{m}^{3}$. Plasma cotinine levels in sperm positive females averaged $68.17 \mathrm{ng} / \mathrm{ml}+/-12.1 \mathrm{ng} / \mathrm{mL}$, though not all potential pregnancies resulted in the delivery of live litters. Plasma cotinine exceeded $50 \mathrm{ng} / \mathrm{ml}$, a level sufficient to designate a human as an active smoker [305]. The values of each of these measures of CSE were below the limit of detection in the Sham chamber. The plasma cotinine levels in the Sham sperm positive dams were below the limit of detection of $4 \mathrm{ng} / \mathrm{mL}$.

In Figure 11A, there was no difference in the weight trajectory between prenatal CSE and Sham offspring. During the first four days after birth, the low-birth-weight phenotype remained evident in the prenatal CSE offspring, after which there was no longer a significant difference between weights in the prenatal CSE and Sham offspring groups. These results demonstrate a phenotype of low-birth-weight with subsequent catch-up growth in offspring exposed in utero to cigarette smoke. In Figure 11B, the serum glucose levels of female CSE offspring were significantly lower compared to female Sham offspring $(p<0.05)$. Serum glucose levels in male offspring were not impacted by prenatal CSE. It should be noted that this decrement in serum 


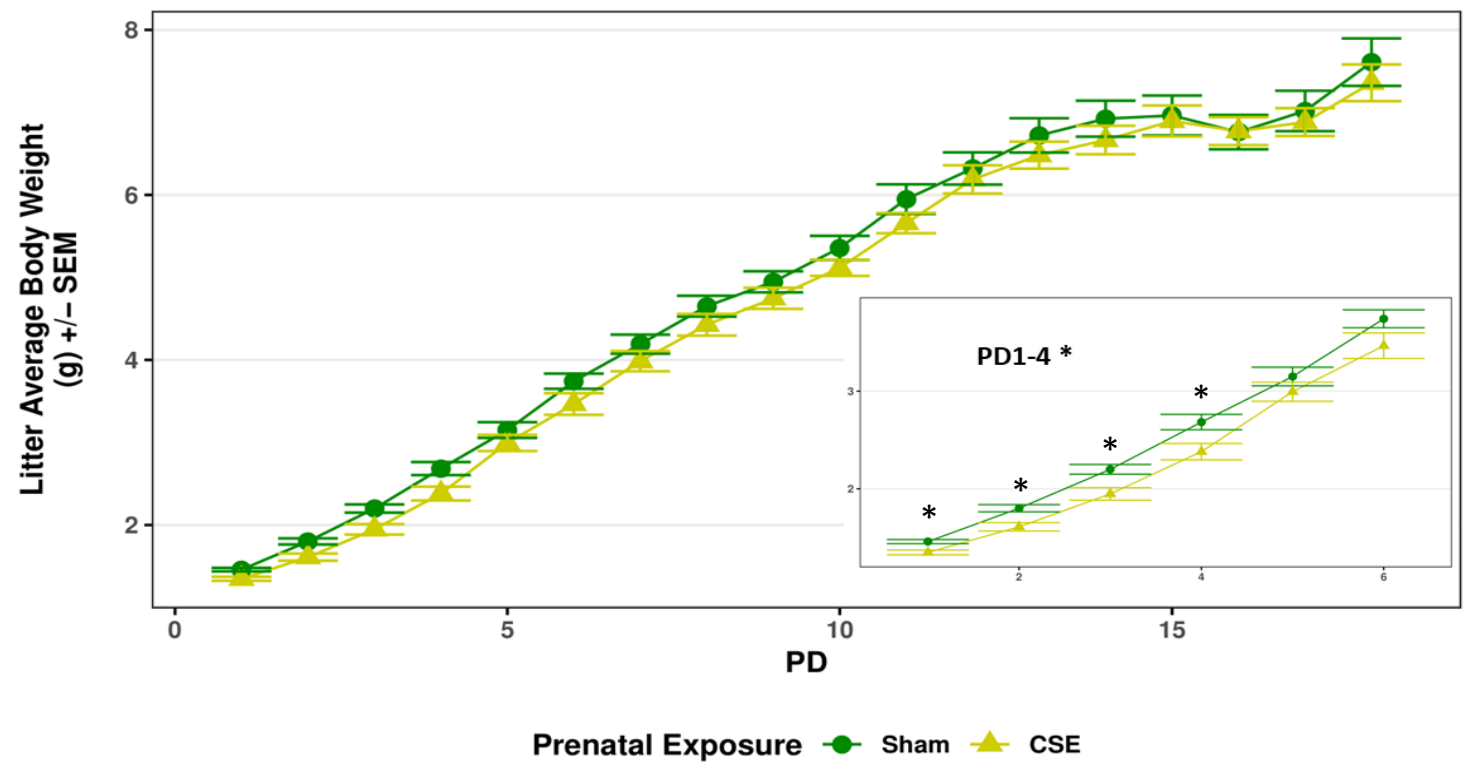

Figure 11A - Offspring exposed in utero to cigarette smoke exhibit a phenotype of low birth weight followed by subsequent catch-up growth - Offspring were subjected to prenatal CSE from GD1-GD19 daily. After birth, the average weight of offspring in each litter was tracked for the first 19 days. The inset chart represents the same data in the main chart but zoomed in on the first six days. CSE offspring had lower birth weight than Sham offspring, which persisted through day $4(p<0.05)$, after which there was no longer a statistical difference between exposure. Significant results had a $p$-value $<0.05$, which is denoted with an ${ }^{*}$ in the figure. 


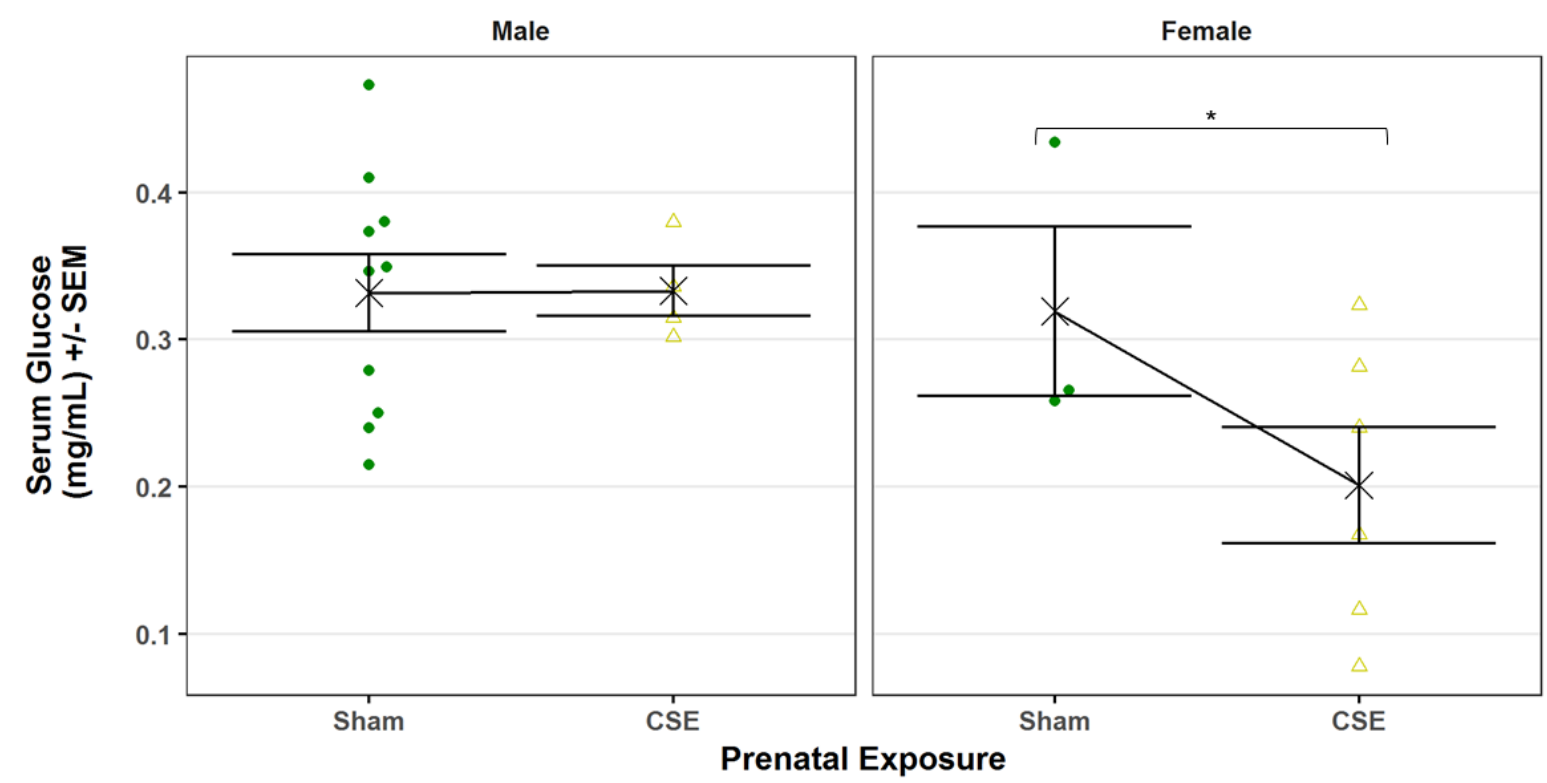

Figure 11B - Female offspring exposed in utero to cigarette smoke exhibit decreased

serum glucose levels - Serum glucose was measured using collected serum from offspring at euthanization via a colorimetric assay. One-way ANOVA $(p=0.03)$ followed by posthoc testing, found that female CSE offspring exhibited decreased serum glucose compared to female Sham offspring $(p<0.05)$. No other differences were detected. Significant results had a $p$-value $<0.05$, which is denoted with an * in the figure. 
glucose levels occurred in a fed state. The key phenotypic changes present in models of in utero cigarette smoke exposure conform to the Barker Hypothesis.

\section{Prenatal CSE Modulates the Acetylation Status of Proteins and Enhances Hepatic SIRT1 and NAMPT Expression in Male Offspring}

In Figure 12, male offspring exposed in utero to cigarette smoke exhibited increased hepatic expression of SIRT1 and NAMPT $(p<0.05)$. A generalized decrease in protein acetylation was evident in both male and female offspring exposed in utero to cigarette smoke $(p$ $<0.05)$. Neither male nor female CSE offspring exhibited any change in the level of acetylated FKHR, a known SIRT1 target protein. The increase in SIRT1 and NAMPT expression in male

CSE offspring support the decrease in acetylated lysine protein residues found. However, female CSE offspring did not exhibit changes in the expression of SIRT1 or NAMPT. Neither male nor female CSE offspring exhibited changes in the expression of PEPCK or HK. These data suggest that the decrease in protein acetylated lysine residues observed in female prenatal CSE offspring is mediated in a manner independent of SIRT1 or NAMPT expression.

\section{Effects of Prenatal CSE on Offspring Hepatic SIRT1 Regulatory Loop and Metabolic Gene Expression Modulated are by Sex}

Examining the effects of prenatal CSE on the hepatic expression of SIRT1 and related metabolic genes revealed marked differences between male and female offspring. In Figure 13A, male offspring exposed to in utero cigarette smoke have increased expression of both Sirt1, Nampt, Pepck, Crtc2, and Pkm2 ( $\mathrm{p}<0.05)$, with a trend toward increased Ehhadh and Cyp7a1 expression $(p<0.1)$. In Figure 13B, female offspring exposed to prenatal CSE exhibited lower expression $(p<0.05)$ of a gluconeogenic gene (Pepck), fat metabolic genes (Ppara, Ehhadh, Mcad, Srebp-1c, Fas), genes involved with FXR signaling (Bsep, Fgfr4, $\beta$-Klotho, Hnf4a, Lxr- $\alpha$, Lrh1, Cyp7a1), and genes in the SIRT1 regulatory loop (Nampt, Fxr, p53). Female offspring subjected to in utero cigarette smoke also saw a trend toward decreased Sirt1, Hk, and Ost- $\beta$ expression (Figure 13B, $\mathrm{p}<0.01$ ). As seen in Figure 11B, the serum glucose levels of female 


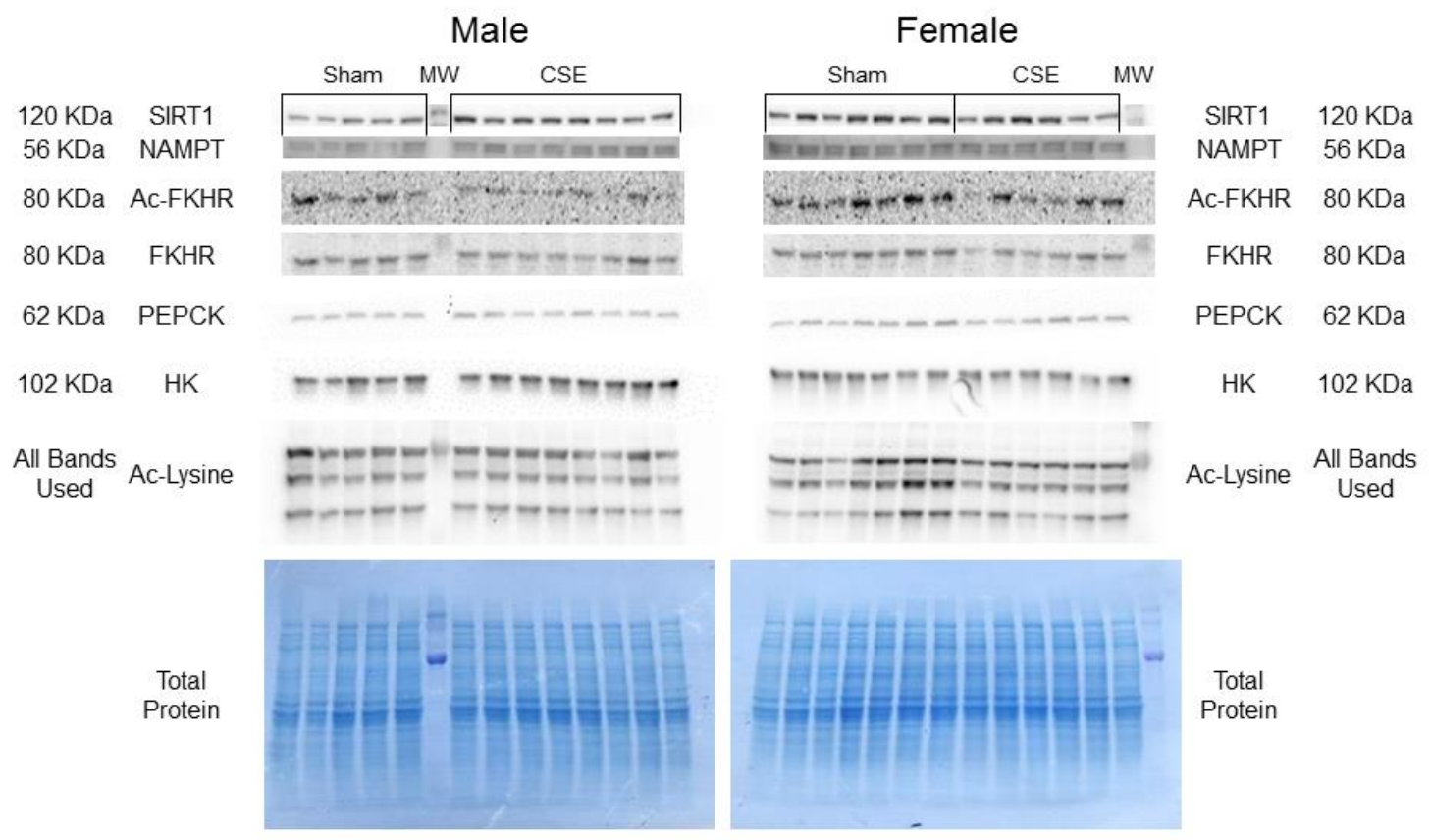

Figure 12A - Exposure in utero to cigarette smoke alters hepatic expression of SIRT1 and NAMPT in male offspring and decreases acetylation of lysine residues in all offspring Protein expression was measured using the livers of male offspring euthanized on either postnatal day 9 or 10 in a fed state. A) Images of Western Blots - Western blots of hepatic protein expression of both male and female offspring with the name of the protein probed in each image and its corresponding molecular weight ( $\mathrm{MW}=$ Molecular weight marker). Band intensities in each lane were normalized to the total protein present in each lane after Coomassie staining. The Coomassie-stained image presented is a representative image of all blots done. 


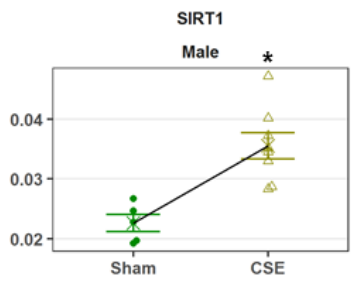

AC-FKHR / FKHR

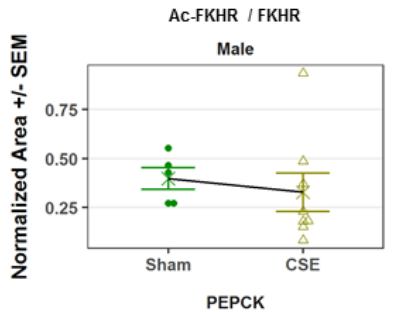

Male

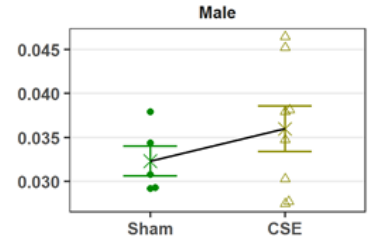

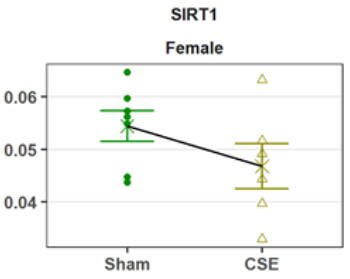

AC-FKHR / FKHR

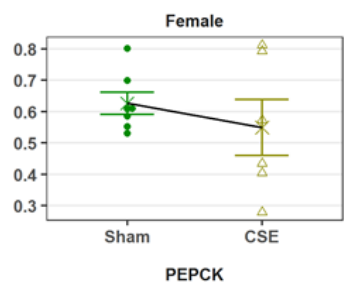

Female

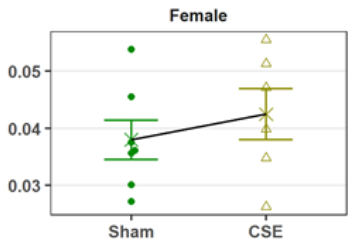

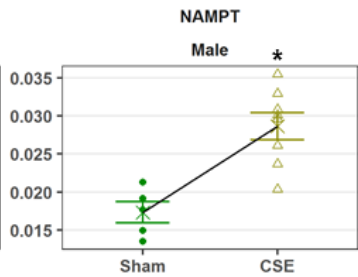

Ac-Lysine
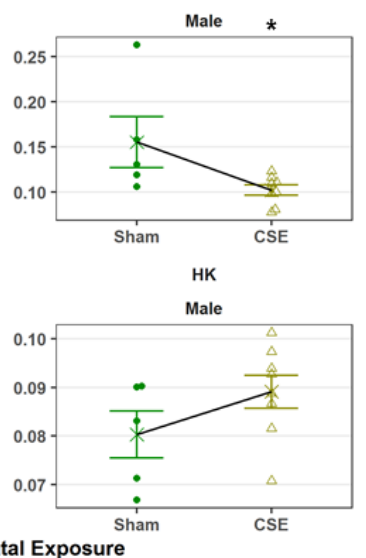

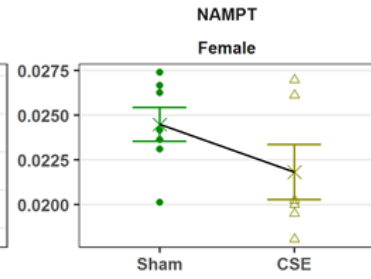

Ac-Lysine

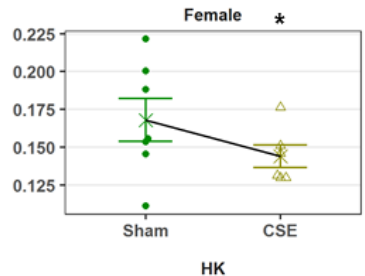

$\mathrm{HK}$

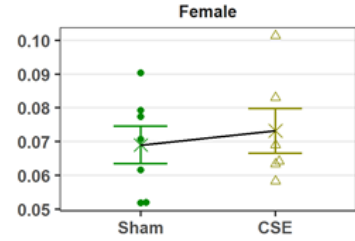

Figure 12 - Exposure in utero to cigarette smoke alters hepatic expression of SIRT1 and NAMPT in male offspring and decreases acetylation of lysine residues in all offspring -

Protein expression was measured using the livers of male offspring euthanized on either postnatal day 9 or 10 in a fed state. B) Quantitation of Western Blots - Prenatal cigarette smoke exposure decreased the hepatic expression of proteins containing acetylated lysine residues. In male offspring exposed to cigarette smoke in utero, the hepatic expression of both SIRT1 and NAMPT was increased, mirroring the increased expression of Sirt1 and Nampt observed. Significant results had a $p$-value $<0.05$, which is denoted with an * in the figure. 


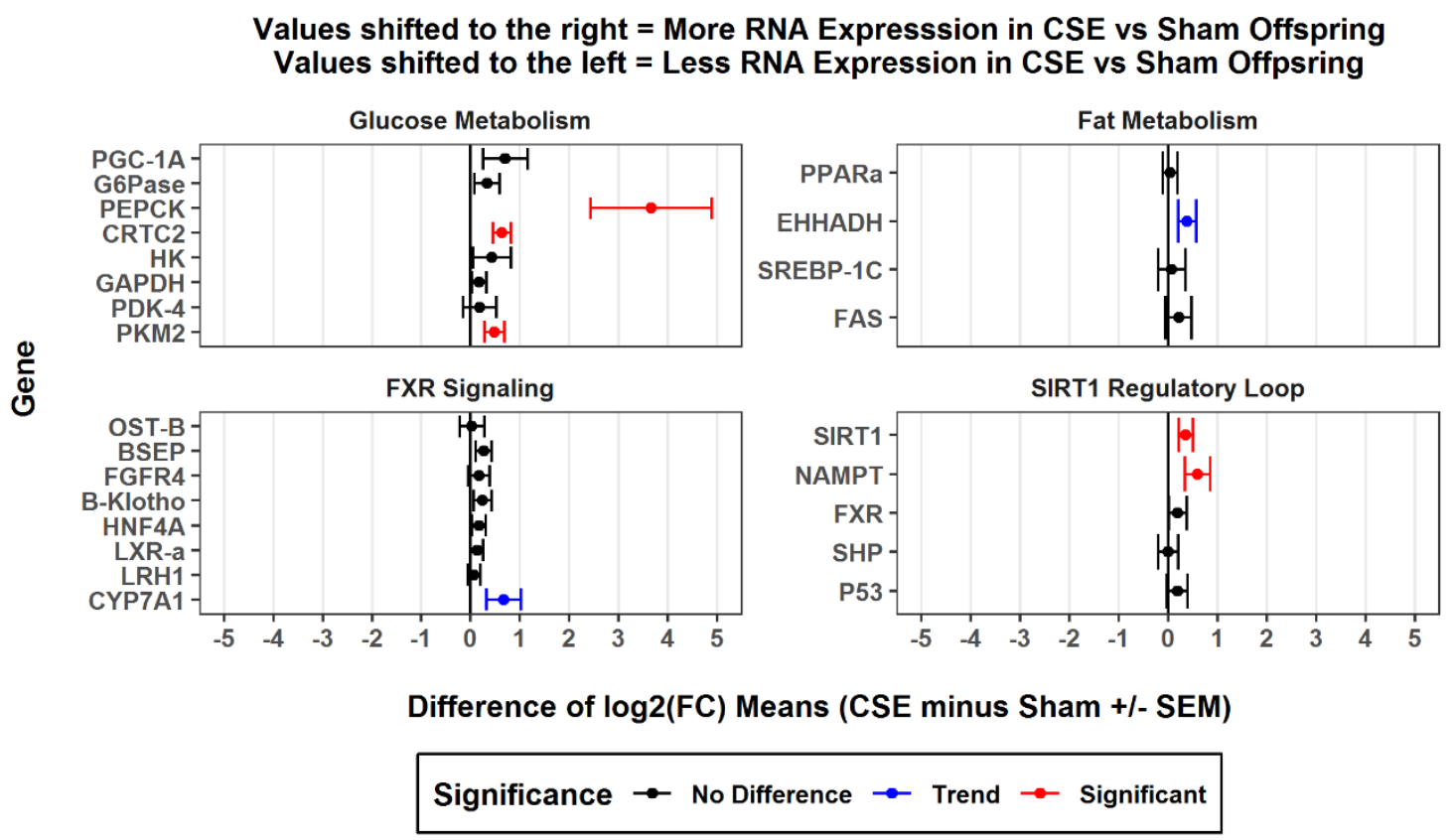

Figure 13A - Prenatal cigarette smoke exposure in male offspring increased hepatic gene expression of Sirt1, Nampt, and key regulatory gluconeogenic enzymes - mRNA expression of genes was measured using the livers of male offspring euthanized on either postnatal day 9 or 10 in a fed state. The expression of Sirt1, Nampt, Pepck, Crtc2, and PKM2 were significantly increased in male offspring in response to in utero cigarette smoke exposure, while Ehhadh and Cyp7a1 exhibited a trend toward increased expression. Results are presented as the difference between the group means of log2 transformed data. Significant results had a p-value $<0.05$ while trends had a $p$-value $<0.1$. 


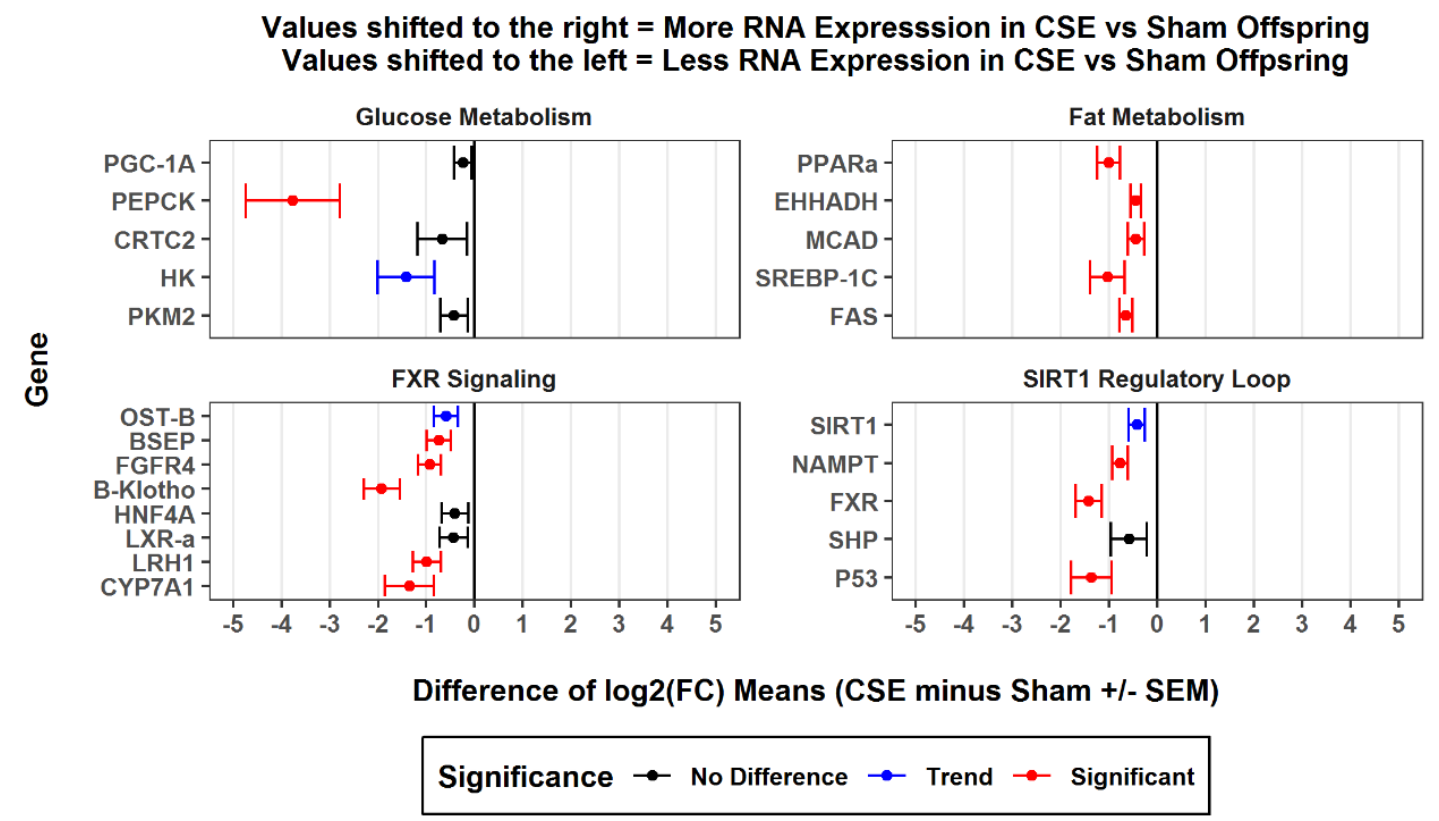

Figure 13B - Prenatal cigarette smoke exposure in female offspring decreased hepatic expression of SIRT1-regulatory loop genes, as well as related groups of metabolic genes mRNA expression of genes, was measured using the livers of female offspring euthanized on either postnatal day 9 or 10 in a fed state. The expression of numerous genes in the SIRT1regulatory loop (not including Sirt1 itself), fat metabolism, FXR signaling, and glucose metabolism was significantly downregulated. Results are presented as the difference between the group means of log2 transformed data. Significant results had a $p$-value $<0.05$ while trends had a $p$ value $<0.1$ 
CSE offspring were lower compared to female Sham offspring, indicating a functional impact of decreased Pepck expression on systemic glucose availability. Overall, it is apparent that female offspring experienced a more extensive array of hepatic gene expression changes after being exposed to cigarette smoke in utero. For genes that were impacted by prenatal CSE in both male and female offspring, the directionality of these changes was in opposition.

\section{Discussion}

In the present current study, offspring subjected to in utero cigarette smoke exhibited a low-birth-weight phenotype. After PD4, there was no longer a significant difference between prenatal CSE and Sham exposures indicating an early catch-up growth. In Chapter II, offspring exposed in utero cigarette smoke also exhibited low birth weight with catch-up growth. The lowbirth-weight phenotype that was observed in our gestation-only active cigarette smoke exposure model is similar to that observed in other models of murine CSE [35-41]. A low-birth-weight phenotype with subsequent catch-up growth was observed in the current study as well as the cohort from Chapter II, which demonstrates that our gestation-only exposure to cigarette smoke consistently induces a phenotype indicating the applicability of the Barker Hypothesis. Unlike models with both pre- and postnatal exposure to cigarette smoke where offspring that exhibited a low-birth-weight and did not exhibit catch-up growth by six months of age [35-40], the offspring in our gestation-only CSE model did exhibit catch-up growth in both male and female offspring.

Passive, or environmental, cigarette smoke exposure throughout gestation in a murine model has also been shown to produce a low birth weight phenotype [41]. The overall duration and dose of cigarette smoke play a role in whether or not catch-up growth occurs in affected offspring.

As described herein, prenatal cigarette smoke exposure affects the hepatic expression of SIRT1 and related metabolic genes in a sex-dependent manner. Male offspring exposed to cigarette smoke in utero exhibited increased hepatic expression of SIRT1 and NAMPT. This observed increase mirrors the increase in hepatic Sirt1 and Nampt in the current study in male offspring subjected to prenatal cigarette smoke. Levels of hepatic proteins with acetylated lysineresidues were also decreased in male offspring in response to prenatal cigarette smoke exposure. 
All this data indicates a generalized decrease in the acetylation of proteins in male offspring during the early neonatal period. There is the probability that the decreased acetylated protein expression observed with in utero cigarette smoke-exposed male offspring in the current study is in part due to other deacetylase enzymes or a decrease in acetylation activity in enzymes such as P300.

Male offspring subjected to prenatal cigarette smoke exposure exhibited increased hepatic expression of both Sirt1 and Nampt. Knowing that Sirt1 is typically upregulated during a fasting response [255], and Nampt promotes SIRT1-induced deacetylation via increased levels of NAD+ [255] and that the offspring from the current study were in a fed state when euthanized, this is indicative of an inappropriately timed fasting response in male offspring when exposed in utero to cigarette smoke. An inappropriately timed fasting response in male offspring in response to prenatal cigarette smoke exposure is further supported by the upregulation of both the gluconeogenic enzyme Pepck and gluconeogenesis-promoting transcription factor Crtc2. Male offspring also exhibited increased Pkm2 when subjected to prenatal cigarette smoke. Pyruvate kinase, which Pkm2 encodes, is an essential final regulatory step for glycolysis and acts as the final step of glycolysis. Normally Pkm2 would be inhibited when gluconeogenic processes are occurring, but the presence of upregulation in both gluconeogenic-related genes and Pkm2 further support the notion of inappropriately timed gluconeogenesis in male offspring when subjected to in utero cigarette smoke. A previous publication from our lab examining hepatic proteomic changes in response to in utero cigarette smoke exposure, lower protein expression of gluconeogenic enzymes Fructose 1,6-Bisphosphatase and Pyruvate Carboxylase was evident in CSE offspring at weaning, but there was only a trend toward decreased activity of those enzymes [35]. However, that study did not examine proteomic changes on a sex-specific basis [35], so those results are not directly comparable to the findings in the current study. Male offspring exposed in utero to cigarette smoke exhibited signs of inappropriately timed-induction of gluconeogenic gene expression. Whether this hepatic molecular phenotype persists into later life stages will be critical in determining how manifestations of the Barker Hypothesis develop in adulthood.

Female CSE offspring in the current study exhibited no changes in hepatic protein expression or acetylated FKHR. However, female CSE offspring did exhibit decreased expression 
of proteins with acetylated-lysine residues. In light of the gene expression changes observed in female prenatal CSE offspring, it appears that there are additional mechanisms at play that are influencing gene expression other than SIRT1 activity by modulation of SIRT1 protein or mRNA levels. SIRT1 is known to have its activity modulated by post-translational modification [339], such as by increasing nuclear localization or increasing its enzymatic activity. SIRT1 also is modulated by factors such as AROS, which, when bound, improves the deacetylase activity of SIRT1 [271]. SIRT1 is negatively regulated by the microRNA miR-34a [262] as well as the long non-coding RNAs MALAT1 [340] and FLRL2 [341]. Regardless of the mechanism, it is evident that female offspring exposed in utero to cigarette smoke possibly have perturbed acetylation or deacetylation processes that impacted the acetylation status of proteins.

Female offspring exposed in utero to cigarette smoke exhibited decreased expression of several Sirt1-related genes, including Nampt, Fxr, and P53, with a trend decrease in Sirt1. Downregulation of FXR-signaling, glucose metabolism, and fat metabolism was also observed in female offspring subjected to prenatal cigarette smoke. We also found that female CSE offspring exhibited decreased serum glucose levels in a fed state. One possibility for these results is that the inherent female resistance predicted by the Barker Hypothesis becomes true later in their lifetime but not before sexual maturity when estrogen signaling in female offspring begins in earnest. Considering how in utero exposure to cigarette smoke induces intrauterine growth restriction $[28,29]$, it is possible that the number of downregulated hepatic gene expression in conjunction with decreased serum glucose levels in a fed state is a reflection of delayed functional liver ontogeny. These results may be reflective of either an underdeveloped liver or a failure in adequately detecting serum glucose or insulin levels, considering the importance of the liver in maintaining serum glucose levels.

Male offspring had a higher number of protein expression changes relative to females in response to in utero cigarette smoke exposure. However, female offspring have a higher number of affected hepatic genes (17 genes in female offspring versus 7 genes in male offspring). These results were surprising considering the Barker Hypothesis suggests that male offspring should be more affected by in utero environmental perturbations compared to female offspring [150]. The 
data presented in the present current study suggests that the sex-specific manifestations of the Barker Hypothesis may be conditional on the life stage being examined. Our findings of in utero CSE-induced sex-dependent hepatic protein and gene expression changes in offspring shortly after birth is novel. These findings represent a step forward in characterizing the impact of maternal smoking during pregnancy on offspring liver molecular phenotype.

At the time of writing, no other published study has examined the sex-specific impact of prenatal cigarette smoke exposure on the expression of genes in the liver shortly after birth. Several studies have looked at gene expression changes occurring in fetal liver tissues [342-344], but not shortly after birth. In one of those studies, prenatal CSE in human fetal liver (11-21 weeks gestation) altered the mRNA expression of enzymes from the 1-carbon cycle such that naturally occurring differences were abolished as well as reducing mRNA transcript levels of IGF2 in male offspring to levels closer to female offspring via increased methylation of related sites [342]. A proteomics study using human fetal liver tissue after 12-16 weeks of gestation showed in response to prenatal CSE affected pathways in glucose metabolism disorder in females and pathways in apoptosis, inflammation, cell proliferation and homeostasis in males [343]. In a study using human fetal liver tissue from the second trimester, prenatal CSE induced increased mRNA expression of phase I metabolic enzymes such as Cyp1a1 and Ephx1 in males in addition to Cyp3a7 and Ephx1 in females [344]. One study using a C67BL/6 model of prenatal cigarette smoke exposure found that there are histological changes that were present 24 hours after birth, such as increased numbers of immune cells and decreased glycogen deposition, but they did not measure gene expression [345]. Together, these studies show that exposure in utero to cigarette smoke has a sex-specific impact on the hepatic molecular phenotype before even the neonatal period examined in the present current study. Future studies with our model should include characterization of the changes to the hepatic molecular phenotype of fetal tissue in order to investigate any association with later life stages. Our current model represents progress in elucidating how hepatic gene expression of metabolically relevant proteins are impacted by in utero exposure to cigarette smoke.

Some limitations were present in the present current study. For example, not tracking individual offspring weight from birth until the end of the study limited the ability to associate the 
magnitude of prenatal CSE-induced low birth weight with specific hepatic molecular phenotypes. In future studies, we plan on taking measures to allow weight tracking across the entire lifetime of offspring to strengthen the outcomes observed. Another limitation was the present current study looking at a single time point. The phenotype that manifests as predicted by the Barker process is one that is long-term in nature and does not manifest fully until adulthood.

Additionally, the changes that were observed in the present current study in the neonatal period begs the question of whether these changes persist into the future or if the hepatic molecular phenotype is altered further. This limitation will be addressed in part in Chapter IV, where siblings of the offspring in the present current that have been weaned were examined. There are also ongoing studies that are being conducted in adult siblings of the offspring in the present current study before and after being subjected to a diet challenge, which will further address the continuity of the observed hepatic molecular phenotype during the neonatal period. Despite these limitations, we believe the data presented here provide new insight into the hepatic molecular phenotype induced by in utero exposure to cigarette smoke in a sex-dependent manner.

We confirmed in our novel study that prenatal cigarette smoke exposure resulted in sexually dimorphic hepatic gene and protein expression alterations with no difference in gross physical appearance in the liver, but histopathological examination has not been done. The lowbirth-weight with subsequent catch-up growth predicted by the Barker Hypothesis was present. Sexually distinct responses to in utero cigarette smoke exposure exist during the early neonatal period. It has been demonstrated that male rodents experience a short-lived surge of androgens on postnatal day 1 [346-348] as well as increased testosterone levels on day 18 of gestation [349]. It should be investigated if prenatal cigarette smoke exposure contributes to the sexually dimorphic changes observed in exposed offspring in the present current study by disrupting these elevations in testosterone in male offspring and contribute to sexually dimorphic changes observed in exposed offspring in our current study. Future studies should examine whether the effects on hepatic gene and protein expressions seen here also are present at subsequent time points in the lifecycle of in utero cigarette smoke-exposed offspring such as at weaning or during adulthood. The effect of current energy status in offspring exposed in utero cigarette smoke should be examined as well to 
confirm if prenatal cigarette smoke results in an inappropriately timed fasting response occur independently of whether offspring are in a fed or fasted state. Our study presents novel insights into the hepatic molecular phenotype induced by in utero exposure to cigarette smoke in a sexdependent manner. 


\title{
CHAPTER IV: IN UTERO EXPOSURE TO CIGARETTE SMOKE EXACERBATES THE
} HEPATIC FASTING RESPONSE IN OFFSPRING AND CAUSES POORLY TIMED FASTING

\author{
RESPONSE IN FEMALE OFFSPRING
}

\section{Introduction}

In $2016,7.2 \%$ of U.S. women reported smoking at any point during pregnancy [47]. This rate of exposure translates to at least 284,000 per year exposed to in utero to cigarette smoke [47]. Although the overall rate of smoking during pregnancy has steadily decreased from the previous rate of $8.4 \%$ overall in 2014 [48] 9.7\% overall in 2008 [49], it is evident that this trend does not fit for all states. Using Kentucky as an example, the rate of smoking during pregnancy was $28.5 \%$ in 1990 [50], 24.5\% in 1999 [50], 25.1\% in 2008 [49], 20.7\% in 2014 [48], and 18.4\% in 2016 [47], showing an overall decrease over time. With an initial examination, it seems that the rate of smoking during pregnancy in West Virginia decreased over time as well, with the rate being $27.8 \%$ in 1990 [50], 26.1\% in 1999 [50], 27.1\% in 2014 [48], and 25.1\% in 2016 [47]. However, examining the rate of smoking during the last 3 months of pregnancy from the PRAMS dataset, the rate varies greatly from $2000-2010$ with the rate ranging from $24.5 \%-31.9 \%$, with a significant overall increase in the rate from the 2000 rate of $24.5 \%$ to the 2010 rate of $30.5 \%$ [328]. It is evident that some states like Kentucky have a significant rate of smoking during pregnancy despite a decrease in the rate over time, while others like West Virginia have a wildly varying rate that does not appear to be significantly decreasing over time. In light of this, it is evident that exposure to cigarette smoke in utero in some states will continue to be a highly significant public health problem for years to come unless drastic measures are taken to curb smoking while pregnant.

In Chapter II, we addressed how the primary outcome of cigarette smoke exposure is low birth weight followed by subsequent catch-up growth, and that this phenotype is a classical outcome with the Barker Hypothesis. In Chapter III, we examined the impact of in utero cigarette smoke exposure on sex-specific low birth weight phenotypes and how this is associated with 
changes in neonatal hepatic SIRT1-related gene and protein expression. However, this leaves unanswered how the impact of in utero cigarette smoke exposure manifests during adolescence. The literature provides several examples of how the impact of exposure in utero to cigarette smoke can be observed during adolescence. For example, a study examining a cohort of 10 -year-old children found that prenatal and postnatal CSE was associated with a lower z-score for height-forage that persisted into adolescence [350]. Another study using a cohort of children in Brazil found that prenatal CSE was associated with decreased height-for-age z-score at birth, one-year-old, 4years-old, 11-years-old, and 15-years old [351]. Another cohort study of children in Brazil found that in utero exposure to cigarette smoke was associated with a more significant change in body mass index between 10 and 17 years old [352]. A study utilizing the Isle of Wight cohort found that the risk of early obesity in children between 1 and 4 years of age that persists to 18 years of age was 2.16 times higher if their mother smoked during pregnancy [353]. Those same children also were at increased risk of other adverse health outcomes at 18 years of age including 2.15 times higher risk for asthma, a 3.2\% decrease in lung function as defined by the FEV1/FVC ratio, and an average elevated systolic and diastolic blood pressure of 11.3 and $12.0 \mathrm{~mm} \mathrm{Hg}$, respectively [353]. It is evident from the literature that prenatal cigarette smoke exposure manifests in perturbed metabolic health not just in adulthood but also during adolescence. However, there have yet to be definitive studies on the molecular phenotype during adolescence as a result of in utero cigarette smoke exposure.

In a study previously conducted by our lab with a mixed-sex murine model using pre- and postnatal exposure to cigarette smoke, our lab observed that exposed offspring at PD21 experience decrements in glucose, lipid, and amino acid metabolic proteins according to hepatic proteomic analysis [35]. It was also found that 6-month-old prenatal CSE offspring had a perturbed expression of metabolic enzymes utilizing the same study design [331]. Specifically, in a fed state, 6-month-old prenatal CSE offspring had decreased levels of hepatic SIRT1 protein as well as increased levels of PEPCK and PGC1 $\alpha$ protein [331]. Prenatal CSE offspring also exhibited oxidative stress in the form of depleted glutathione levels and experienced decreased serum glucose levels [331]. In Chapter II, we observed that 9-month old male offspring subjected to in 
utero cigarette smoke exposure exhibited decreased hepatic SIRT1 protein expression while female offspring were unaffected. In Chapter III, we demonstrated that 1.5-week old male offspring exposed in utero to cigarette smoke exhibited increased expression of hepatic SIRT1 protein and mRNA while female CSE offspring exhibited no change in SIRT1 protein and a trend toward decreased Sirt1 mRNA expression with decrements in many SIRT1-related genes. We also observed in Chapter III that female CSE offspring exhibited a decrement in serum glucose levels despite being in a fed state. Despite all this data previously generated by our lab, the hepatic molecular phenotype manifested due to prenatal cigarette smoke exposure still has not been examined on a sex-specific basis or how it is modulated by a fed versus a fasted state.

There are several pressing questions to be answered still. Are the perinatal sex-specific changes observed in Chapter III still present after weaning? Do the changes observed depend on the current fasting status of the offspring? Does the sex of the offspring modulate the impact of prenatal CSE on metabolic function? Does prenatal cigarette smoke exposure result in a perturbed fed or fasted state response? Do female CSE offspring still exhibit a decrement in serum glucose levels? Answering these questions would allow for a more thorough understanding of the increased risk of metabolic diseases in exposed offspring and facilitate the possible development of targeted early-life interventions and is the aim of the present current chapter.

The objective of this study was to characterize the sex-specific impact of prenatal CSE on hepatic SIRT1 expression and on target gene expression in weanling mice, at a time which low birth weight is no longer evident and have not yet reached full sexual maturity (19 days of age), in either a fed or fasted state. We utilized an established mouse inhalation model of cigarette smoke exposure that mimics active maternal smoking during pregnancy throughout the entire fetal development period (GD1-GD19). The mRNA gene expression levels for SIRT1 targets, which are indicative of activation or suppression, are indirect indicators of SIRT1 activity. The offspring in the present current study are siblings to the neonatal offspring examined in Chapter III.

\section{Methods}

\section{Animal Exposure}


Female C57BL/6 mice maintained on breeder chow (LabDiet 5015) were mated overnight and checked the following day for the presence of vaginal sperm. Sperm positive status was considered gestational day (GD) 0 for the exposure timing. On GD1, female C57BL/6 mice were placed into either a cigarette smoke exposure (CSE) chamber or a sham exposure (ambient filtered room air) chamber for 6 hours/day daily from GD1 to GD19. Cigarette smoke exposure was carried out using a Teague TE-10C exposure apparatus (Teague Industries) smoking Marlboro Red cigarettes, which was the most commonly smoked cigarette at the time of model development, using the FTC method of smoking (2-second puff per minute). After parturition, dams with litters were maintained on breeder chow. Offspring were weaned by PD19. On PD19, representative offspring of each litter were euthanized by carbon dioxide asphyxiation either immediately or following a $\sim 4$ hour fast. At euthanization, the excised liver was frozen on dry ice and stored at $-80^{\circ} \mathrm{C}$.

\section{Cigarette Smoke Exposure Monitoring via Measurement of Carbon Monoxide, Total Suspended Particulates, and Plasma Cotinine}

At the start of each day of exposure, the level of $\mathrm{CO}$ within the cigarette smoke exposure chamber was allowed to increase rapidly by fully closing the dilutor valve until the level reached 110 parts per million (ppm) as detected with a commercially available carbon monoxide detector with a digital readout. Once this level was reached, $\mathrm{CO}$ levels were further adjusted by opening and closing the dilutor valve as appropriate to maintain a target CO level ranging between 110 to $150 \mathrm{ppm}$. At the end of exposure each day, the dilutor valve was fully opened to allow rapid dissipation of any remaining $\mathrm{CO}$ in the cigarette smoke exposure chamber. TSPs were recorded twice daily by drawing air from each exposure chamber via a sampling port through a preweighed piece of filter paper. The filter paper was then reweighed, and the pre- and post-sampling difference was divided by the volume of air drawn through the sampling device in a five minute interval In order to quantitate the magnitude of cigarette smoke exposure, cotinine, the primary metabolite of nicotine, was measured in representative females immediately after cessation of the

exposure period on days 14-18. Tail vein blood was collected from dams within 30 minutes of the 
end of exposure into heparinized capillary tubes and was subsequently centrifuged at $10,000 \times g$ for 10 minutes. The collected plasma fractions were stored at $-20^{\circ} \mathrm{C}$ until ready for cotinine measurement. Using the Cotinine One-Step ELISA Detection Kit (International Diagnostic Systems; St. Joseph, MI), the collected plasma fractions were assayed for the concentration of cotinine following the manufacturer's instructions. The absorbance of the reaction substrate was measured at $450 \mathrm{~nm}$ using a SpectraMax 190 Microplate Reader (Molecular Devices, San Jose, $\mathrm{CA}$ ) and reported as nanograms per milliliter. Levels of plasma cotinine greater than or equal to $50 \mathrm{ng} / \mathrm{mL}$ were considered sufficient to simulate active maternal smoking during pregnancy.

\section{Offspring Health and Serum Glucose Measurement}

Animals were housed and maintained at the University of Louisville Research Resources Center, an Association for Assessment and Accreditation of Laboratory Animal Care accredited facility. All protocols were approved by the UofL Institutional Animal Care and Use Committee. Cages for animal housing were changed weekly, unlimited food and water provided, and maintained in an environment with a 12-hour light/dark cycle with controlled temperature and humidity. Offspring were weighed daily and checked for health indicators such as levels of activity and eye-opening. Blood was collected from offspring after euthanization via cardiac puncture using heparinized syringes. Blood samples were subsequently centrifuged at 10,000x $g$ for 10 minutes, and the separated plasma fraction was collected and stored at $-80^{\circ} \mathrm{C}$.

Using the Glucose (HK) Assay Kit (Catalog \# GAHK20; Sigma-Aldrich; St. Louis MO), the collected plasma fractions were diluted 50 -fold and were assayed for the concentration of glucose following the manufacturer's instructions. The absorbance of the reaction substrate was measured at $340 \mathrm{~nm}$ using a SpectraMax 190 Microplate Reader (Molecular Devices, San Jose, $\mathrm{CA}$ ) and reported as $\mathrm{mg}$ glucose $/ \mathrm{mL}$.

\section{Western Blotting}

Liver was homogenized in ice-cold buffer [100 mM PO4 Buffer, $150 \mathrm{NaCl}, 0.1 \%$ Tween, $0.1 \%$ SDS, $0.5 \mathrm{M}$ Tris $\mathrm{pH} 8.0,2$ tabs per $10 \mathrm{~mL}$ phosphatase and protease inhibitor cocktails, 10 
$\mu \mathrm{L}$ per $10 \mathrm{~mL}$ EDTA, $7.5 \mathrm{mg} / \mathrm{mL}$ dithiothreitol (DTT)] using a Tissue-Tearor homogenizer in 2second on/off pulses over the span of 1 minute. The concentration of protein was measured by the Bradford method [301]. Liver homogenates were mixed with Laemmli buffer (0.25M Tris $\mathrm{pH}$ 6.8, glycerol, $10 \%$ SDS, trace bromophenol blue), reduced with DTT $(1 \mathrm{~g} / 5 \mathrm{~mL})$ then heated at $70^{\circ}$ C for 10 minutes. Thirty-five $\mu \mathrm{g}$ total protein was applied to $10 \%$ acrylamide gels with separation performed at $100 \mathrm{~V}$ for 2 hours in Tris-glycine buffer (25 mM Tris, $192 \mathrm{mM}$ glycine, $\mathrm{pH}$ 8.3) followed by transfer to PVDF membrane at 70V for 2 hours in a non-reducing buffer [Tris-Glycine buffer (25 mM Tris, $192 \mathrm{mM}$ glycine, pH 8.3) with 20\% ethanol]. Following blocking in 5\% non-fat dry milk in TBS-T solution (Tris-Buffered Saline-Tween, $137 \mathrm{mM} \mathrm{NaCl}, 20 \mathrm{mM}$ Tris-Base pH 7.6, $0.1 \%$ Tween-20), the blots were incubated overnight at $4^{\circ} \mathrm{C}$ with primary antibody (SIRT1 1/500, Santa Cruz \#sc-15404; PEPCK 1/500, Santa Cruz \#sc-271029; HK 1/500, Cell Signaling \#2024; NAMPT 1/1000, Thermo-Fisher \#PA5-23198; ac-lysine 1/250, Millipore \#AB3879; ac-FKHR 1/250, Santa Cruz \#sc-49437; FKHR 1/500, Santa Cruz \#sc-11350) diluted in non-fat dry milk in TBS-T. After three washes of 10 minutes each in TBS-T, blots were incubated with secondary antibody complexed to horseradish peroxidase (anti-mouse, Thermo \#32430; anti-rabbit, Cell Signaling \#7074S) in non-fat dry milk diluted in TBS-T at room temperature for 3 hours. After three successive washes in TBS-T, blots were developed with LumiGLO chemiluminescent reagent and peroxide (Cell Signaling Technology \#7003S, Danvers, MA), and visualized with a Bio-Rad Gel Doc XR+ system (Bio-Rad Laboratories, Hercules, CA). Following visualization, blots were washed briefly in TBS-T and incubated with Coomassie stain $(0.1 \%$ Coomassie Brilliant Blue G250 Dye in 1:1 methanol:water) for 10 minutes, followed by two 10 minute washes in destaining solution (5:4:1 ethanol:water:acetic acid), and a final wash for 5 minutes in deionized water. Blots were briefly air-dried and scanned for quantitation of total protein.

\section{Real-Time Quantitative PCR (RT-qPCR) for mRNA Expression}

At euthanization, a section of the liver was equilibrated overnight in RNAlater at $4^{\circ} \mathrm{C}$, after

which it was placed into long term storage at $-80^{\circ} \mathrm{C}$. A portion of RNAlater-stabilized liver was used for RNA extraction using the Qiagen RNeasy Mini Kit (Qiagen, Germantown, MD) per 
manufacturer's instructions. The final elution was carried out using two aliquots of nuclease-free water and was stored at $-80^{\circ} \mathrm{C}$. RNA concentration was determined using a NanoDrop 8000 Spectrophotometer (Thermo Scientific, Waltham, MA). Using two $\mu \mathrm{g}$ of each isolated RNA sample as input, cDNA was synthesized using the High-Capacity cDNA Reverse Transcription Kit following the manufacturer instructions (Applied Biosystems cat\# 4368813). cDNA reactions were carried out as $20 \mu \mathrm{L}$ reactions in PCR tubes placed in a Bio-Rad MyCycler (Bio-Rad Laboratories, Hercules, CA). The cDNA reactions were set for an initial 10 minutes at $25^{\circ} \mathrm{C}$, followed by 2 hours at $37^{\circ} \mathrm{C}$ and 5 minutes at $85^{\circ} \mathrm{C}$. The cDNA reaction tubes were held at $4^{\circ} \mathrm{C}$ until placed into storage at $-20^{\circ} \mathrm{C}$.

Mouse 18S rRNA gene was used as the reference gene for RT-qPCR. The genes of interest have the forward and reverse primer sequences listed in Table 2. PCR reactions were carried out as $10 \mu \mathrm{L}$ reactions in 96 well plates and ran using a Bio-Rad iQ5 Multicolor Real-Time PCR Detection System (Bio-Rad Laboratories, Hercules, CA) in 96 well plates. The PCR reactions were set for an initial $3 \mathrm{~min}$ at $95^{\circ} \mathrm{C}$, followed by 40 cycles of $15 \mathrm{sec}$ at $95^{\circ} \mathrm{C}$ and $1 \mathrm{~min}$ at $60^{\circ} \mathrm{C}$ using SsoAdvanced Universal SYBR Green Supermix (Bio-Rad Laboratories, Hercules, CA). Each PCR plate was filled out with three technical replicates per sample and three template-free controls per primer pair. Data were analyzed using the $\Delta \Delta C T$ method followed by $\log 2$ transformation of fold-change values.

\section{Real-Time Quantitative PCR (RT-qPCR) for miRNA Expression}

At euthanization, a section of the liver was equilibrated overnight in RNAlater at $4^{\circ} \mathrm{C}$, after which it was placed into long term storage at $-80^{\circ} \mathrm{C}$. A portion of RNAlater-stabilized liver was used for miRNA extraction using the Qiagen miRNeasy Mini Kit (Qiagen, Germantown, MD) per manufacturer's instructions. The final elution was carried out using two aliquots of nuclease-free water and was stored at $-80^{\circ} \mathrm{C}$. RNA concentration was determined using a NanoDrop 8000 Spectrophotometer (Thermo Scientific, Waltham, MA). Using two $\mu \mathrm{g}$ of each isolated total RNA sample as input, cDNA was synthesized using the miScript II RT Kit following the manufacturer instructions using miScript HiSpec Buffer included in the kit to selectively convert mature miRNA 


\begin{tabular}{|c|c|c|c|}
\hline \# & Name & Forward Primer & Reverse Primer \\
\hline 1 & SIRT1 & GCAGGTTGCGGGAATCCAA & GGCAAGATGCTGTTGCAAA \\
\hline 2 & NAMPT & CATAGGGGCATCTGCTCATT & GCTGCTGGAACAGAATAGCC \\
\hline 3 & PGC-1A & ATGTGTCGCCTTCTTGCTCT & ATCTACTGCCTGGGGACCTT \\
\hline 4 & CRTC2 & GCCACATTGACAGTTCTCCA & AAGTGTCTTGGGGGTTAGGG \\
\hline 5 & SREBP-1C & ATGGATTGCACATTTGAAGACATGCTC & CCTGGGCTGCTGGGGCCTG \\
\hline 6 & PPARa & AGAAGTTGCAGGAGGGGATT & TCGGACTCGGTCTTCTTGAT \\
\hline 7 & FGF21 & CAAATCCTGGGTGTCAAAGC & ATTGTAACCGTCCTCCAGCA \\
\hline 8 & FGFR4 & GACCAAACCAGCACCGTGGCTGTGAAGATG & GTTTCCCTGGCGGCACATTCCACAATCAC \\
\hline 9 & B-Klotho & CGAGCCCATTGTTACCTTGT & CTCCAAAGGTCTGGAAGCAG \\
\hline 10 & CYP7A1 & CAGGGAGATGCTCTGTGTTCA & AGGCATACATGCAAAACCTCC \\
\hline 11 & CYP8B1 & TTCGACTTCAAGCTGGTCGA & CAAAGCCCCAGCGCCT \\
\hline 12 & SHP & AAGGGCACGATCCTCTTCAA & CTGTTGCAGGTGTGCGATGT \\
\hline 13 & BSEP & CTGCCAAGGATGCTAATGCA & CGATGGCTACCCTTTGCTTC \\
\hline 14 & OSTB & GATGCGGCTCCTTGGAATTA & GGAGGAACATGCTTGTCATGAC \\
\hline 15 & GAPDH & AAATGGTGAAGGTCGGTGTGAAC & CAACAATCTCCACTTTGCCACTG \\
\hline 16 & PEPCK & TATGCTGATCCTGGGCATAA & CACGTTGGTGAAGATGGTGT \\
\hline 17 & G6Pase & TCTGTCCCGGATCTACCTTG & GTAGAATCCAAGCGCGAAAC \\
\hline 18 & HK & TATGAAGACCGCCAATGTGA & ПTTCCGCCAATGATCTTTTC \\
\hline 19 & PKM2 & TCGCATGCAGCACCTGATT & CCTCGAATAGCTGCAAGTGGTA \\
\hline 20 & EHHADH & CCGGTCAATGCCATCAGT & CTAACCGTATGGTCCAAACTAGC \\
\hline 21 & FAS & GCTGCGGAAACTTCAGGAAAT & AGAGACGTGTCACTCCTGGACTT \\
\hline 22 & MCAD & GGTTTGGCTTTGGACAATG & TGACGTGTCCAATCTACCACA \\
\hline 23 & FXR & TCCACAACCAAGTTTTGCAG & TCTCTGTTTGTTGTACGAATCCA \\
\hline 24 & LXR-a & TGAGAGCATCACCTTCCTCA & TGGAGAACTCAAAGATGGGG \\
\hline 25 & LRH1 & AACGATGTCCCTACTGTCGATT & CATGCGGTCGGCTCTTAC \\
\hline 26 & HNF4A & CAGCAATGGACAGATGTGTGA & TGGTGATGGCTGTGGAGTC \\
\hline 27 & IL-6 & CTGCAAGAGACTTCCATCCAG & AGTGGTATAGACAGGTCTGTTGG \\
\hline 28 & MCP-1 & CAGGTCCCTGTCATGCTTCT & GAGGATCACCAGCAGCAGGT \\
\hline 29 & TNF-a & AAGCCTGTAGCCCACGTCGTA & AGGTACAACCCATCGGCTGG \\
\hline 30 & COL1A1 & TAGGCCATTGTGTATGCAGC & ACATGTTCAGCTTTGTGGACC \\
\hline 31 & PDK-4 & GCCTTGGGAGAAATGTGTGT & CACTGGCTTITTGAGTGCAA \\
\hline 32 & P53 & GTCACAGCACATGACGGAGG & TCTTCCAGATGCTCGGGATAC \\
\hline 33 & $18 \mathrm{~S}$ & CTCAACACGGGAAACCTCAC & CGCTCCACCAACTAAGAACG \\
\hline 34 & miR-34a & GCAGTGTCTTAGCTGGTTG & AATCGAGCACCAGTTACG \\
\hline 35 & miR-152 & AGTGCATGACAGAACTTGG & AATCGAGCACCAGTTACG \\
\hline
\end{tabular}

Table 2: Forward and reverse primer pairs for RT-qPCR 
(Qiagen, Germantown, MD). cDNA reactions were carried out as $20 \mu \mathrm{L}$ reactions in PCR tubes placed in a Bio-Rad MyCycler (Bio-Rad Laboratories, Hercules, CA). The cDNA reactions were set for 60 minutes at $37^{\circ} \mathrm{C}$ and 5 minutes at $95^{\circ} \mathrm{C}$. The cDNA reaction tubes were held at $4^{\circ} \mathrm{C}$ until placed into storage at $-20^{\circ} \mathrm{C}$.

Mouse miR-152 miRNA gene was used as the reference gene for RT-qPCR for miRNA expression. The miRNA gene of interest have the forward and reverse primer sequences listed in Table 2. PCR reactions were carried out as $10 \mu \mathrm{L}$ reactions in 96 well plates and ran using a BioRad iQ5 Multicolor Real-Time PCR Detection System (Bio-Rad Laboratories, Hercules, CA) in 96 well plates. The PCR reactions were set for an initial 3 min at $95^{\circ} \mathrm{C}$, followed by 40 cycles of 15 sec at $95^{\circ} \mathrm{C}$ and $1 \mathrm{~min}$ at $60^{\circ} \mathrm{C}$ using SsoAdvanced Universal SYBR Green Supermix (Bio-Rad Laboratories, Hercules, CA). Each PCR plate was filled out with three technical replicates per sample and three template-free controls per primer pair. Data were analyzed using the $\Delta \Delta C T$ method followed by log2 transformation of fold-change values.

\section{Imputation of Missing Weight Data}

Offspring weights were recorded from PD1 (the day after birth) until weaning (PD19). Occasionally, throughout the neonatal monitoring period, recording of daily weighs was missed. In order to utilized linear mixed-effects modeling, imputation was performed. Imputation was accomplished using the mice $\mathrm{R}$ package [338] by the predictive mean matching method utilizing a value of $\mathrm{m}$, the number of datasets to impute, of 50 with 100 max iterations. At the end of the imputation procedure, all 50 potential imputed values generated for each missing value were averaged together and merged back with the original dataset to render it complete. This completed dataset was then used for offspring weight analyses.

\section{Linear Mixed-Effects Modeling}

Linear mixed-effects modeling using the Ime4 $\mathrm{R}$ package [302] was conducted to assess the effect of prenatal CSE on hepatic protein and mRNA expression levels. The general mixedeffects equation utilized to model the data is given by: 
Eq. 1: Variable of Interest $\sim 1+$ Exposure $+(1 \mid$ Cohort $)$

Where Exposure indicates modeling of fixed effects for prenatal exposure. The (1/Cohort) term indicates the identity of the cohort set as a random intercept. The random intercept was set this way to account for the fact that each individual offspring is more likely to be similar to other littermates than offspring from different litters. The goal of this was to help control potential confounding of results due to potential natural variance between litters. In instances where all individuals within each comparison to be done were all from the same cohort, the following equation was used instead:

Eq. 2: Variable of Interest $~ 1+$ Exposure

The above equation 2 is similar to 1 but without the (1|Cohort) random intercept term. For some analyses, fitting random-effects models were not possible due to the occurrence of singular fits. For the models using Eq. 1 listed above that resulted in a singular fit, Eq. 2 was instead used. Post-hoc testing was conducted using t-tests for specific a priori contrasts of interest using the finalized models with the emmeans R package [303]. The contrast of interest for all RT-qPCR experiments was CSE versus Sham. For analysis of RT-qPCR data, log2 transformed foldchange values were used while Western blot analysis used protein expression values normalized by total protein.

\section{$\underline{\text { Results }}$}

\section{Cigarette Smoke Exposure Parameters and Low-Birth Weight Phenotype}

The carbon monoxide level in the cigarette smoke exposure chamber was $147 \mathrm{ppm}+/-$ $43 \mathrm{ppm}$ while total suspended particulates averaged $26.8 \mathrm{mg} / \mathrm{m}^{3}+/-7.5 \mathrm{mg} / \mathrm{m}^{3}$. Plasma cotinine levels in sperm positive females were $68.17 \mathrm{ng} / \mathrm{ml}+/-12.1 \mathrm{ng} / \mathrm{ml}$. Plasma cotinine exceeded 50 $\mathrm{ng} / \mathrm{ml}$, a level sufficient to designate a human as an active smoker [305]. The values of each of 
these measures of CSE were below the limit of detection in the Sham chamber. The plasma cotinine levels in the Sham sperm positive dams were below the limit of detection of $4 \mathrm{ng} / \mathrm{mL}$.

The data present in Figure 14 is from siblings of offspring from Chapter III. In Figure 14, there was no difference in the weight trajectory between prenatal CSE and Sham offspring. As mentioned in Chapter III, low birth weight was evident in the prenatal CSE offspring that persists until day 5, after which there is no longer a significant difference between prenatal CSE and Sham offspring groups. There continued to be no difference between prenatal CSE and Sham offspring at PD19 when the offspring have been weaned. There was also no difference in serum glucose levels between prenatal exposure groups at PD19 (data not shown). These results demonstrate a phenotype of low birth weight with subsequent catch-up growth in offspring exposed in utero to cigarette smoke with no detectable difference after weaning. These results recapitulate fundamental phenotypic changes present in models of in utero cigarette smoke exposure and what is predicted by the Barker Hypothesis.

\section{Offspring subjected to in utero cigarette smoke exposure modulated hepatic expression of SIRT1, NAMPT, and acetylated proteins in a sex- and fasting status-specific manner}

We examined the effects of in utero cigarette smoke exposure on hepatic expression of SIRT1 and related proteins in weanling offspring in both a fed and fasted state. As shown in Figure 15, the hepatic expression of NAMPT was decreased in male CSE offspring in a fed state $(p<0.05)$ while the expression of SIRT1, PEPCK, HK, acetylated-FKHR, and acetylated-lysine residues were unchanged. As shown in Figure 16, male offspring in a fasted state and subjected to in utero cigarette smoke exposure exhibited increased hepatic expression of SIRT1 and decreased expression of NAMPT $(p<0.05)$. Male CSE offspring in a fasted state also exhibited a trend toward decreased expression of FKHR and proteins with acetylated lysine residues $(p<$ 0.10). Hepatic expression of PEPCK and HK were unchanged in male CSE offspring in a fasted state.

In Figure 15, female CSE offspring in a fed exhibited increased hepatic expression of SIRT1 and decreased expression of NAMPT $(p<0.05)$ with a trend toward increased HK 


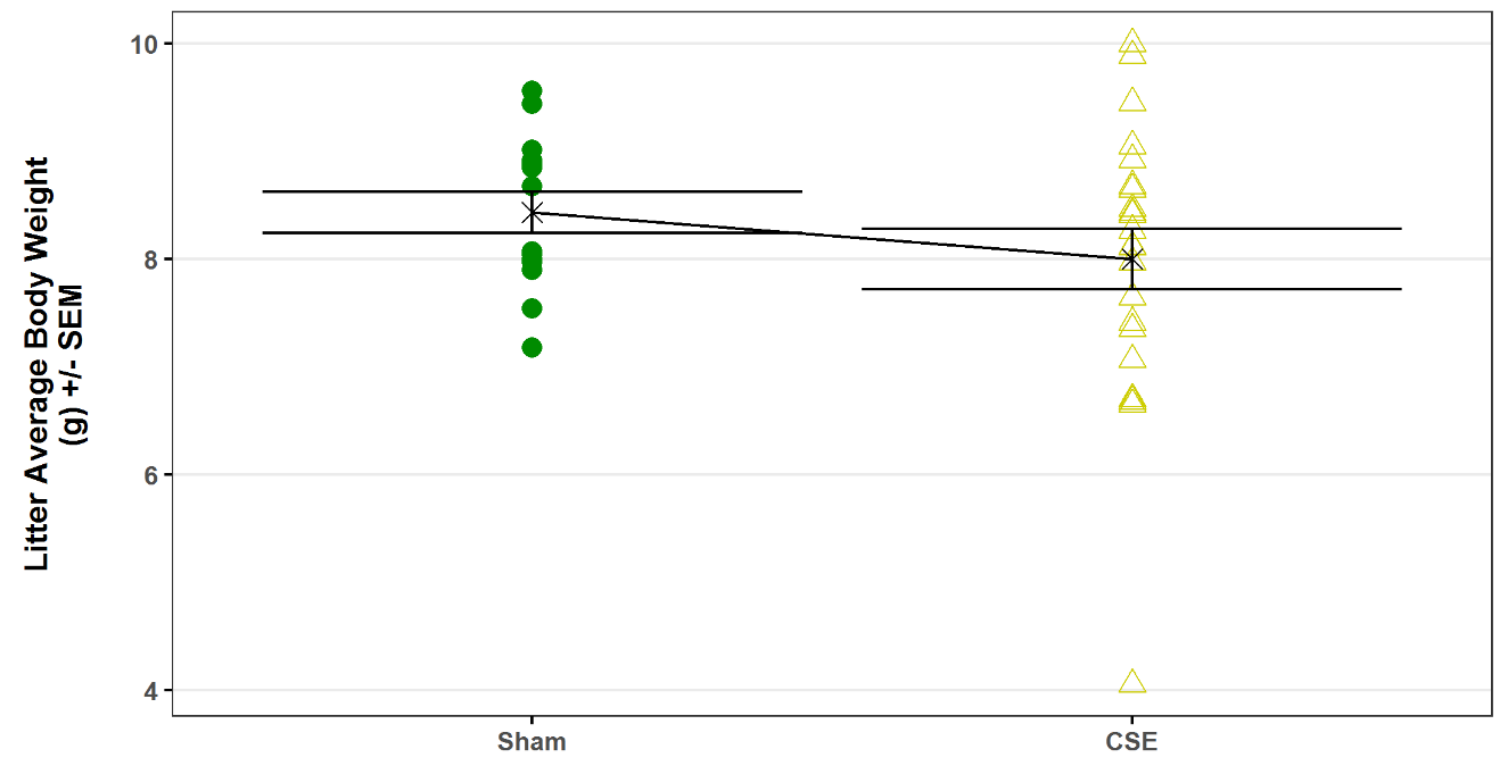

Figure 14 - Offspring exposed in utero to cigarette smoke exhibit catch-up growth by weaning - Offspring were subjected to prenatal CSE from GD1-GD19 daily for 6 hours. After birth, the average weight of offspring in each litter was tracked each postnatal day (PD). CSE offspring exhibited no difference from Sham offspring on PD19. 


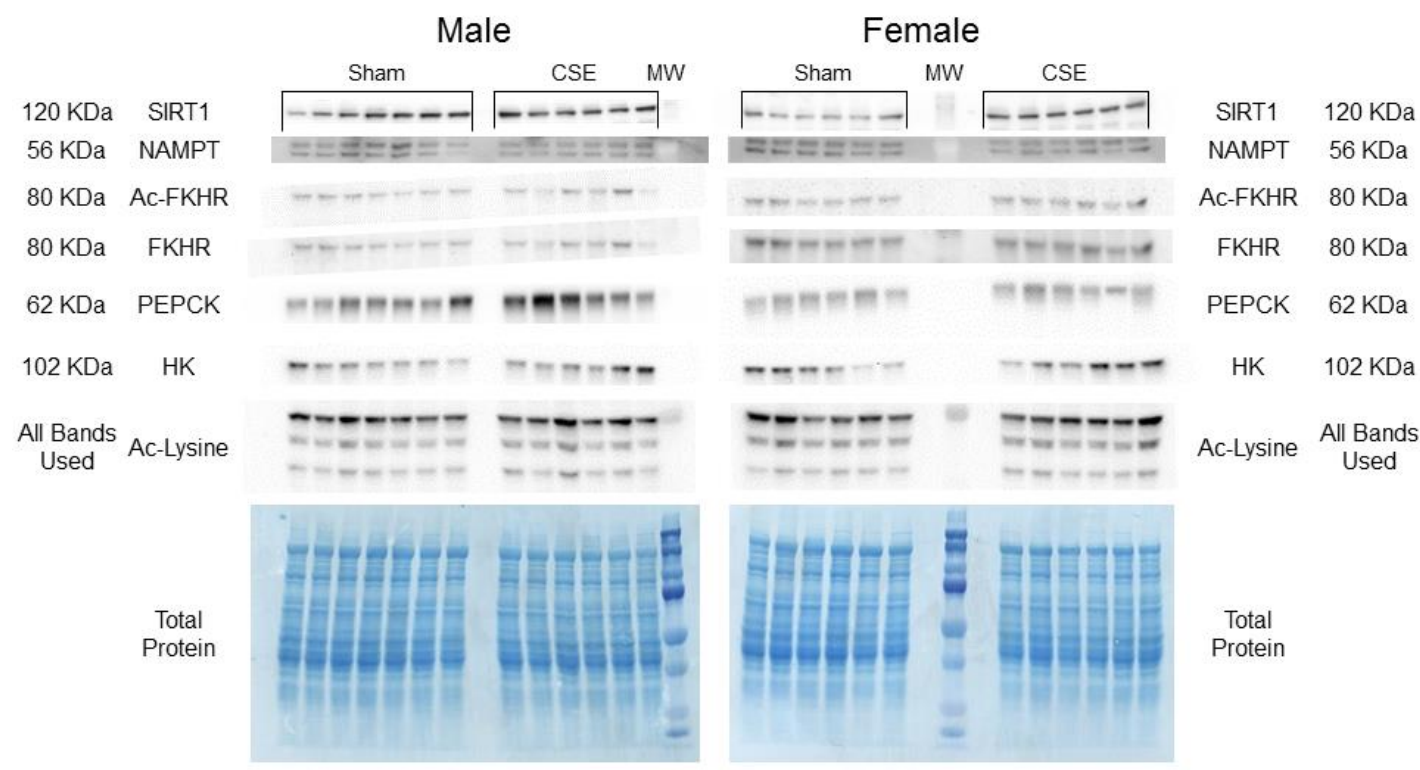

Figure 15 - Exposure in utero to cigarette smoke increased expression of SIRT1 in fed female offspring and decreased hepatic expression of NAMPT in all fed offspring - Protein expression was measured using the livers of male offspring euthanized on postnatal day 19 in a fed state. A) Images of Western Blots - Western blots of hepatic protein expression of both male and female offspring with the name of the protein probed in each image and its corresponding molecular weight (MW = Molecular weight marker). Band intensities in each lane were normalized to the total protein present in each lane after Coomassie staining. The Coomassie-stained image presented is a representative image of all blots done. 

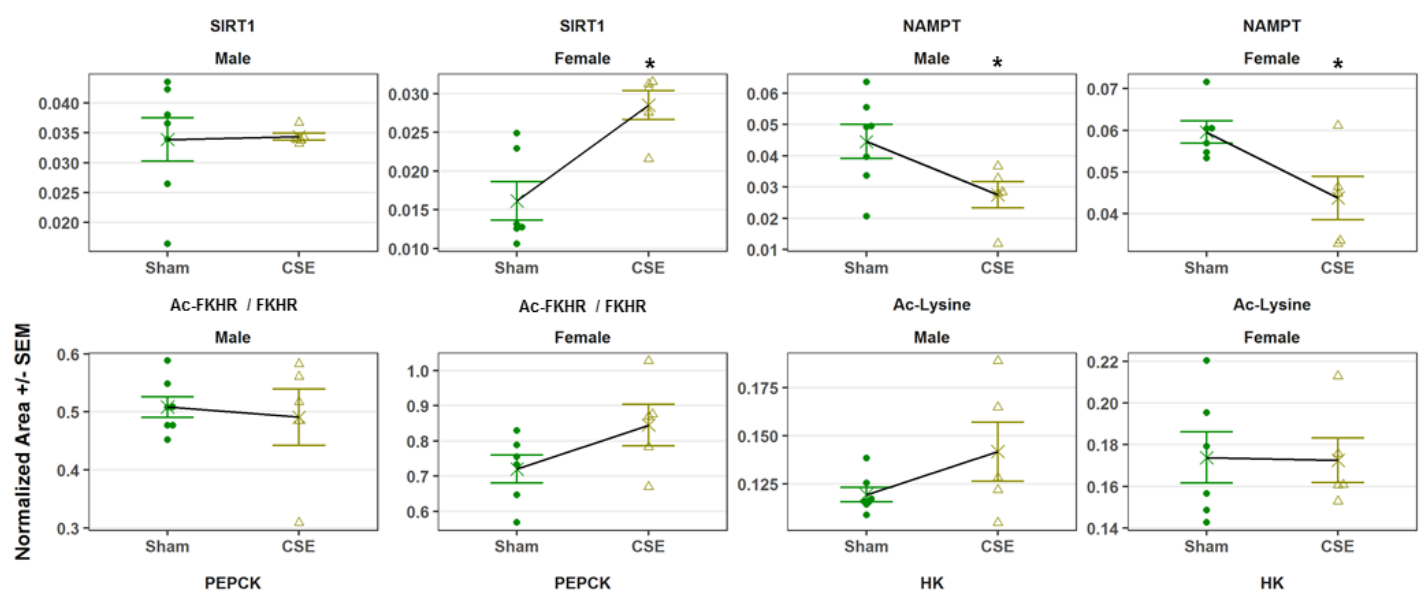

AC-FKHR / FKHR
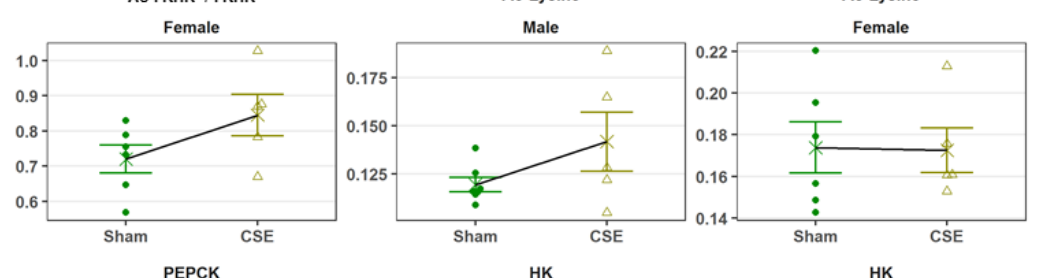

Male

Female
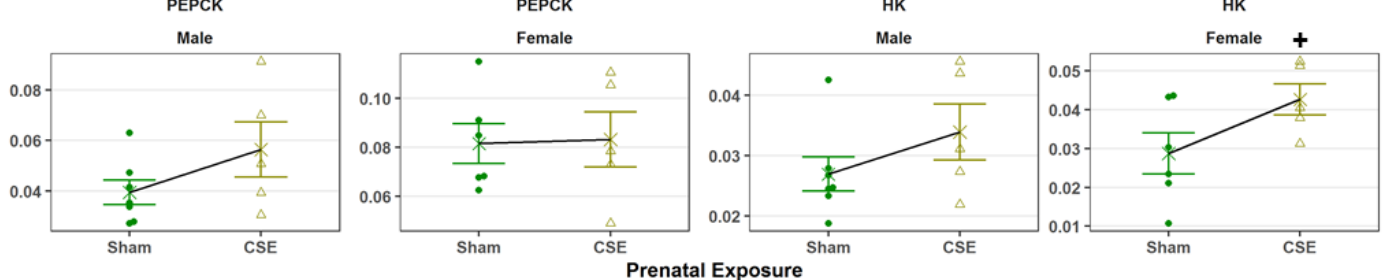

Figure 15 - Exposure in utero to cigarette smoke increased expression of SIRT1 in fed female offspring and decreased hepatic expression of NAMPT in all fed offspring- Protein expression was measured using the livers of male offspring euthanized on postnatal day 19 in a fed state. B) Quantitation of Blots - In all offspring in a fed state, in utero exposure to cigarette smoke decreased the expression of NAMPT. In fed female CSE offspring, the expression of SIRT1 was increased while the expression of HK exhibited a trend toward increased expression. Significant results had a p-value $<0.05$, which is denoted with an * in the figure. Results exhibited a trend that had a $p$-value $<0.10$, which is denoted with $a+$ in the figure. 


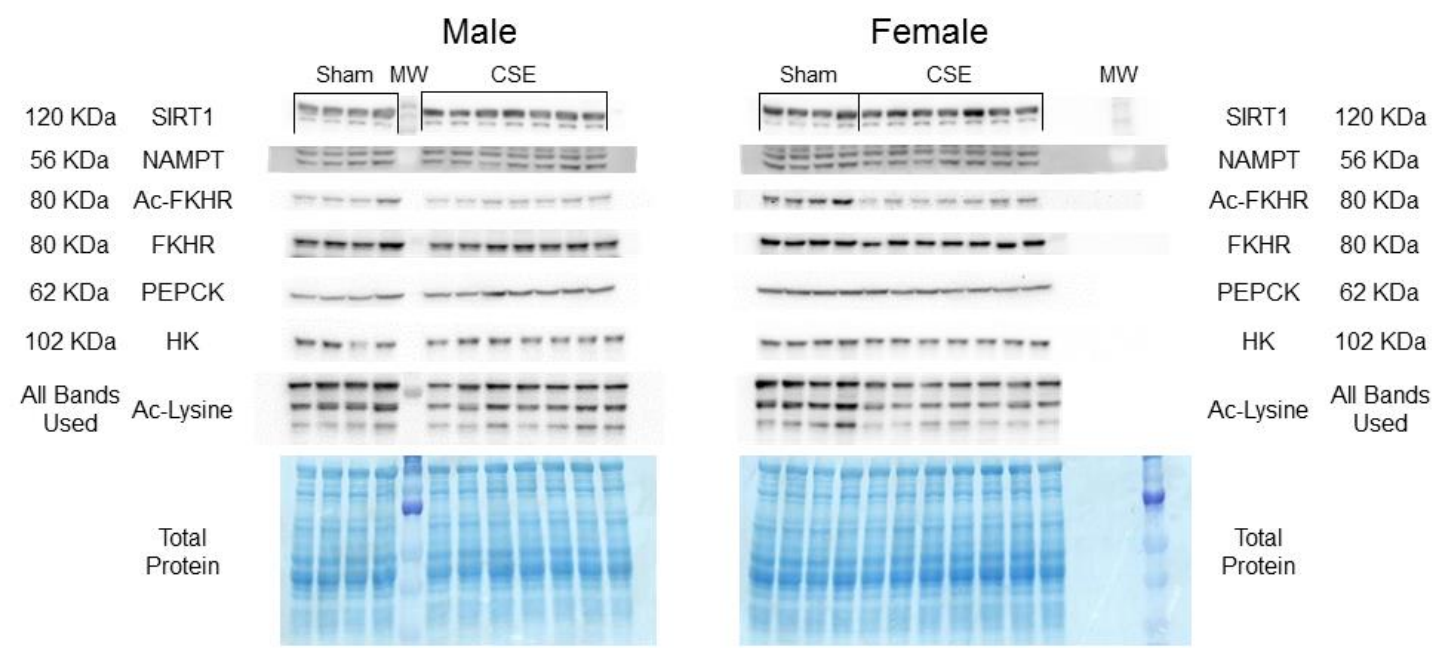

Figure 16 - Exposure in utero to cigarette smoke altered hepatic expression of SIRT1 and NAMPT in fasted male offspring - Protein expression was measured using the livers of male offspring euthanized on postnatal day 19 in a fasted state. A) Images of Western Blots Western blots of hepatic protein expression of both male and female offspring with the name of the protein probed in each image and its corresponding molecular weight ( $\mathrm{MW}=$ Molecular weight marker). Band intensities in each lane were normalized to the total protein present in each lane after Coomassie staining. The Coomassie-stained image presented is a representative image of all blots done. 


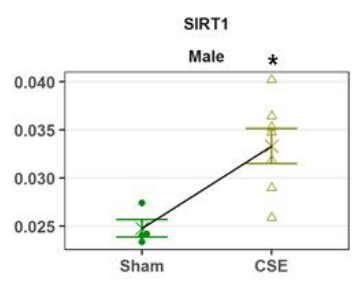

AC-FKHR / FKHR

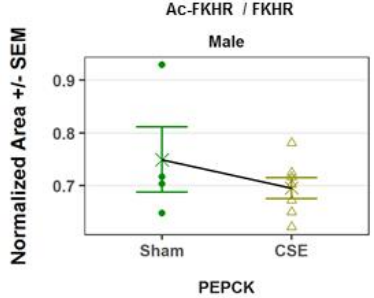

Male

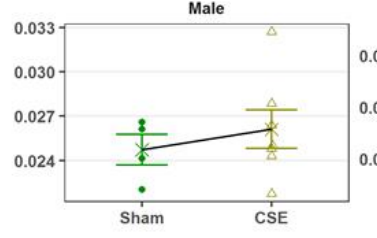

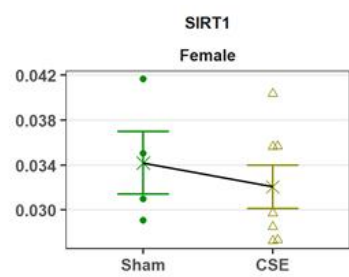

Ac-FKHR / FKHR

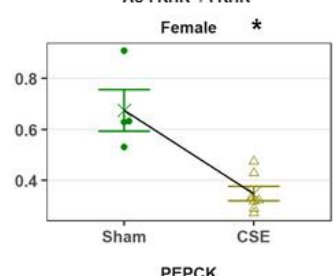

PEPCK

Female

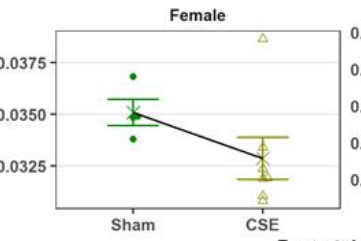

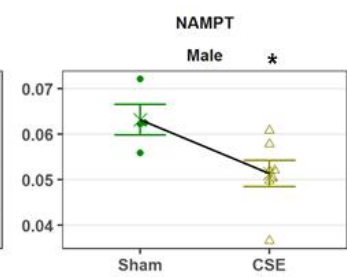

Ac-Lysine

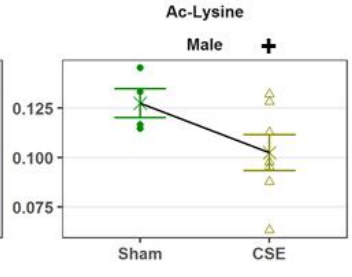

HK

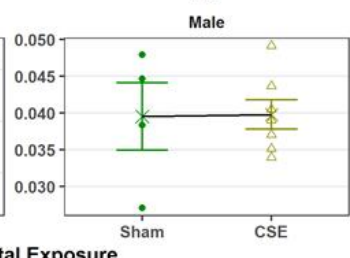

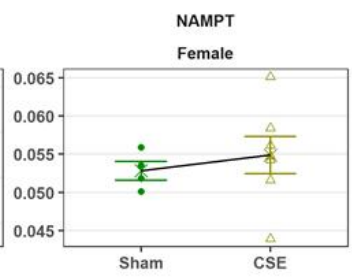

Ac-Lysine

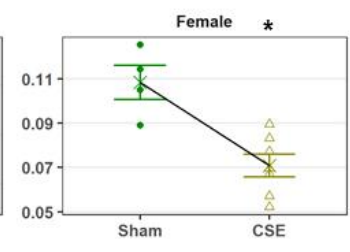

HK

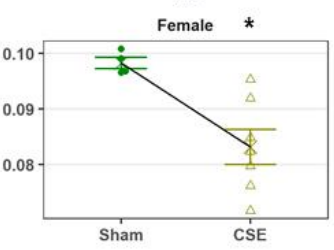

Figure 16 - Exposure in utero to cigarette smoke altered hepatic expression of SIRT1 and NAMPT in fasted male offspring - Protein expression was measured using the livers of male offspring euthanized on postnatal day 19 in a fasted state. B) Quantitation of Blots - Fasted male CSE offspring exhibited increased hepatic expression of SIRT1 and decreased expression of NAMPT. Fasted male CSE offspring also exhibited a trend toward decreased expression of proteins with acetylated lysine residues. Fasted female offspring exposed in utero to cigarette smoke exhibited decreased expression of HK, acetylated-FKHR/FKHR, and proteins with acetylated lysine residues. Significant results had a $p$-value $<0.05$, which is denoted with an * in the figure. Results exhibited a trend that had a $p$-value $<0.10$, which is denoted with $a+$ in the figure. 
expression $(p<0.05)$. Hepatic expression of PEPCK, HK, ac-FKHR, and acetylated-lysine residues were unchanged in female CSE offspring in a fed state. In Figure 16, female CSE offspring exhibited decreased hepatic expression of acetylated FKHR without a concomitant decrease in FKHR as well as decreased expression of $\mathrm{HK}$ and proteins with acetylated lysine residues $(p<0.05)$. Female CSE offspring in a fasted state exhibited no difference in the hepatic expression of SIRT1, NAMPT, or PEPCK. While both male and female CSE offspring in a fed state exhibited decreased hepatic NAMPT protein expression, only female CSE offspring exhibited an increase in SIRT1 expression. Conversely, male CSE offspring in a fasted state had SIRT1 and NAMPT protein expression that mirrored that observed in female CSE offspring in a fed state. Female CSE offspring in a fasted state were the only offspring to exhibit decreased hepatic expression of ac-FKHR, acetylated lysine residues, and HK in response to in utero cigarette smoke exposure. The data suggest that female offspring in a fasted that have been subjected to cigarette smoke exposure in utero exhibit facets of an exaggerated fasting response that is independent of SIRT1 liver expression. The current study demonstrates how in utero cigarette smoke exposure resulted in sex-specific alteration in the hepatic gene and protein expression of SIRT1 and related proteins.

\section{Offspring exposed to in utero cigarette smoke exhibited altered hepatic expression of Sirt1 and related genes in the SIRT1 regulatory loop according to sex and fasting status}

To better contextualize the alterations in the protein expression of SIRT1 and related metabolic proteins observed in offspring subjected to in utero cigarette smoke exposure, we measured the mRNA expression of Sirt1 as well as related genes in the SIRT1 regulatory loop. In Figure 17A, fed male CSE offspring exhibited increased hepatic expression of $\operatorname{Sirt1}(p<0.05)$ and a trend toward increased expression of $\operatorname{Fxr}(\mathrm{p}<0.1)$. Male CSE offspring in a fed state exhibited no differences in hepatic mRNA expression for Fxr, Shp, or P53. In Figure 18A, fasted male CSE offspring exhibited a trend toward decreased hepatic expression of Sirt1 and P53 $(\mathrm{p}<$ 0.10). Male CSE offspring in a fasted state exhibited no difference in hepatic mRNA expression for Nampt, Fxr, or Shp. Male CSE offspring also exhibited no difference in the hepatic expression 


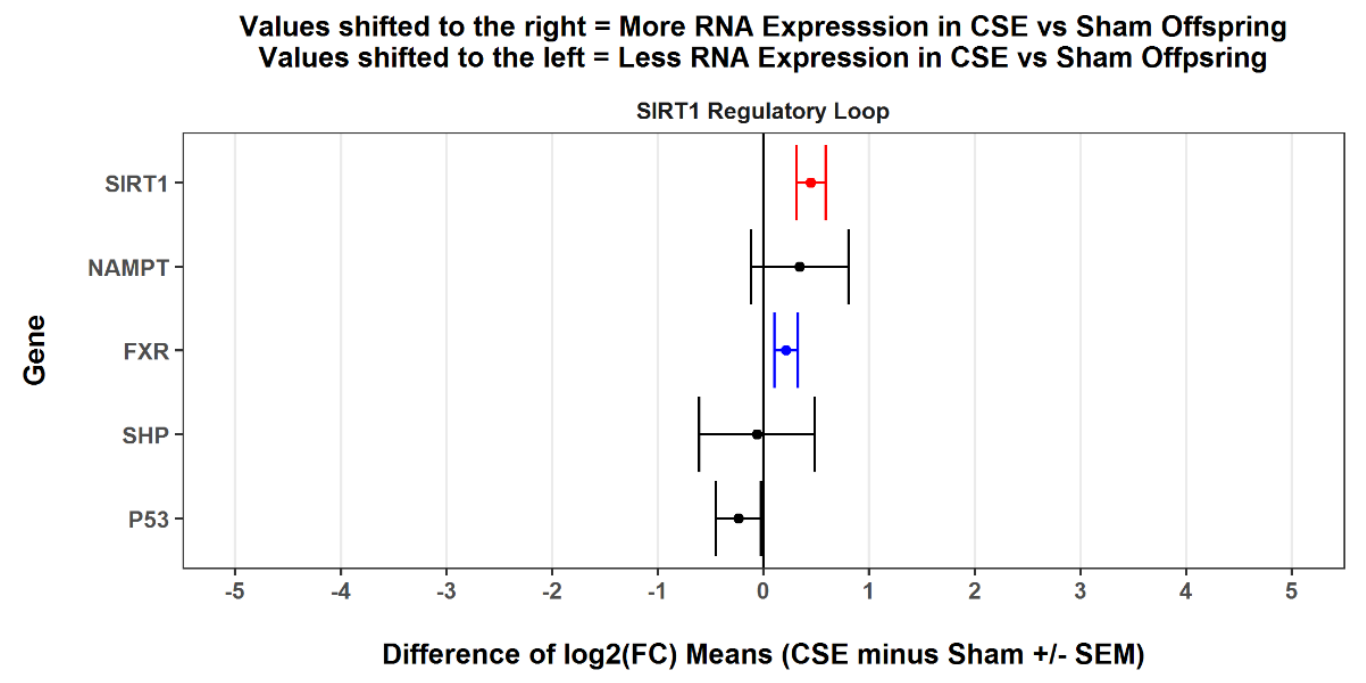

Significance $\rightarrow$ No Difference $\rightarrow$ Trend $\rightarrow$ Significant

Figure 17 - Prenatal cigarette smoke exposure in fed offspring increased hepatic gene expression of Sirt1 and modulates the expression of other related enzymes by sex - mRNA expression of genes was measured using the livers of offspring euthanized on postnatal day 19 in a fed state. A) Fed Male Offspring - The expression of Sirt1 was significantly increased in fed male offspring in response to in utero cigarette smoke exposure, while $F x r$ exhibited a trend toward increased expression. Results are presented as the difference between the group means of $\log 2$ transformed data. Significant results had a $p$-value $<0.05$ while trends had a $p$-value $<$ 0.1 . 


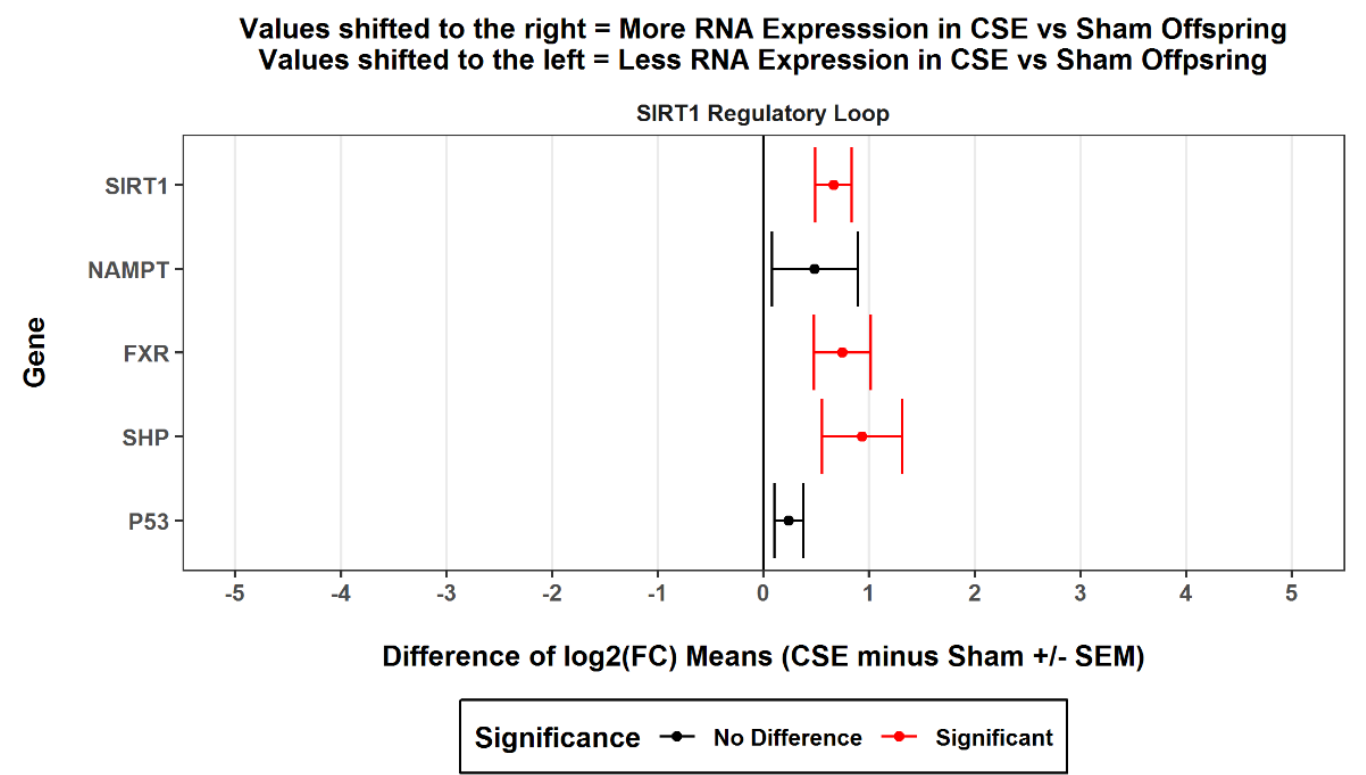

Figure 17 - Prenatal cigarette smoke exposure in fed offspring increased hepatic gene expression of Sirt1 and modulates the expression of other related enzymes by sex - mRNA expression of genes was measured using the livers of offspring euthanized on postnatal day 19 in a fed state. B) Fed Female Offspring - Fed female CSE offspring exhibited increased expression of Sirt1, Fxr, and Shp. Results are presented as the difference between the group means of $\log 2$ transformed data. Significant results had a p-value $<0.05$. 


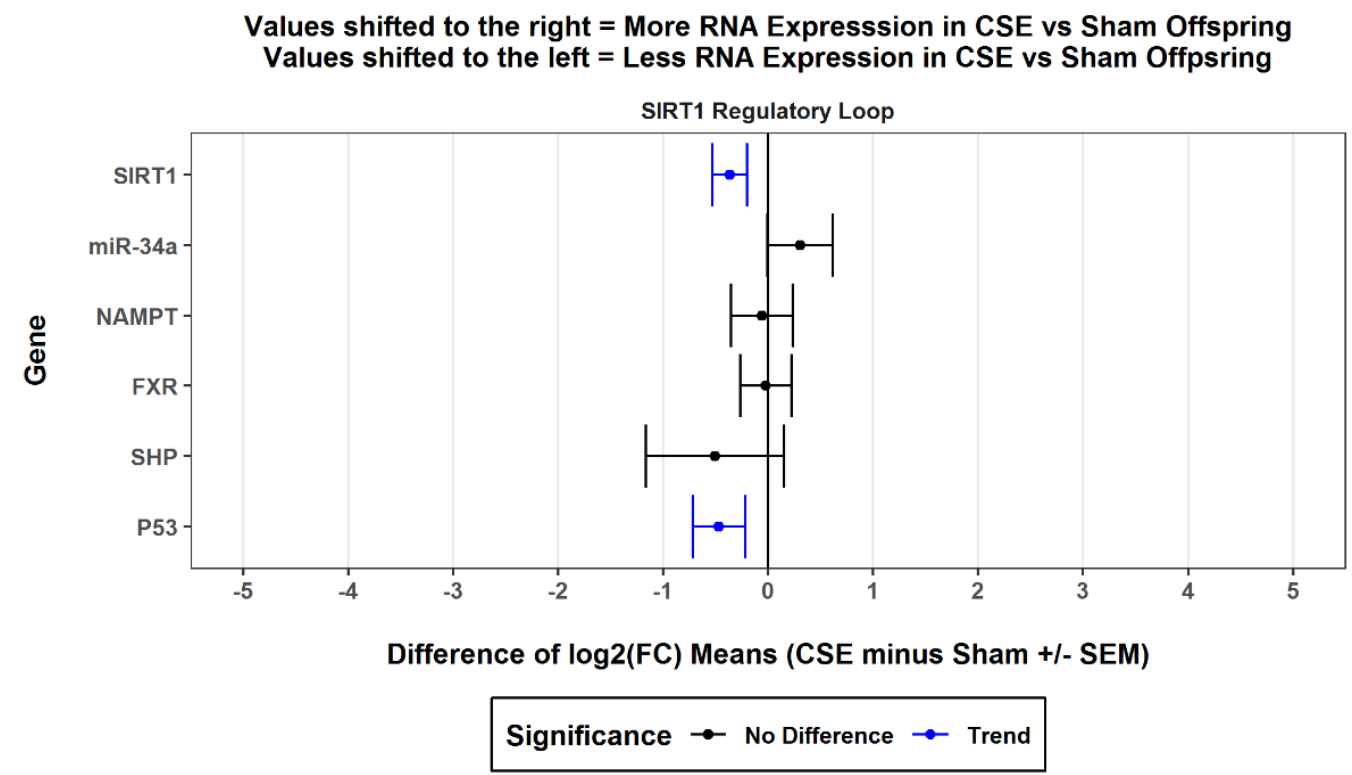

Figure 18 - Prenatal cigarette smoke exposure in fasted offspring modulated hepatic expression of SIRT1 regulatory loop based on sex- mRNA expression of genes was measured using the livers of offspring euthanized on postnatal day 19 in a fasted state. A) Fasted Male Offspring - In fed male offspring subjected to in utero cigarette smoke exposure, Sirt1 and P53 exhibited a trend toward decreased hepatic expression. Results are presented as the difference between the group means of log2 transformed data. Trending results had a pvalue $<0.1$ 


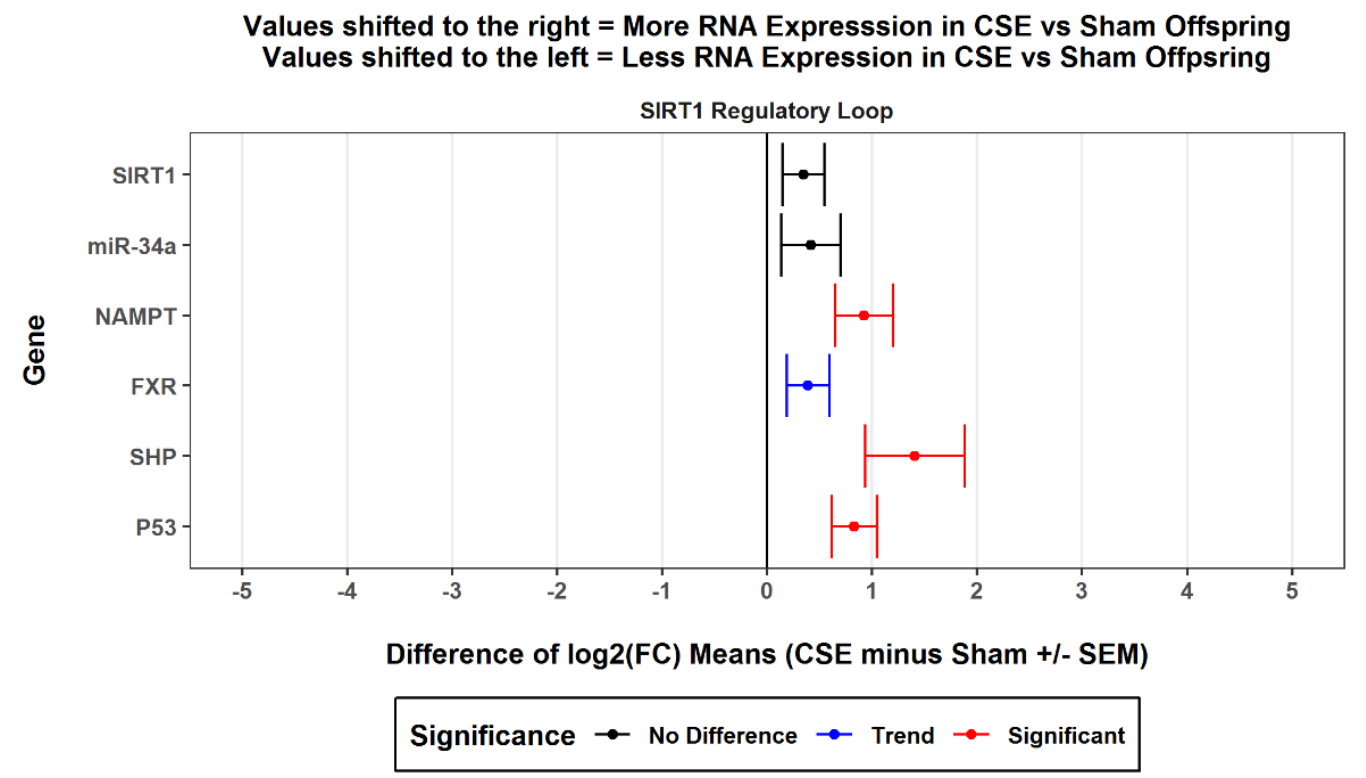

Figure 18 - Prenatal cigarette smoke exposure in fasted offspring modulated hepatic expression of SIRT1 regulatory loop based on sex-mRNA expression of genes was measured using the livers of offspring euthanized on postnatal day 19 in a fasted state. B) Fasted Female Offspring - Fasted female CSE offspring exhibited increased expression of Nampt, Shp, and P53, and exhibited a trend toward increased Fxr expression. Results are presented as the difference between the group means of log2 transformed data. Significant results had a p-value $<0.05$ while trends had a p-value $<0.1$. 
of the miRNA miR-34a.

As seen in Figure 17B, fed female CSE offspring exhibited increased hepatic expression of Sirt1, Fxr, and Shp $(\mathrm{p}<0.05)$. Female CSE offspring in a fed state exhibited no difference in hepatic mRNA expression for Nampt, or P53. As seen in Figure 18B, fasted female offspring exhibited increased hepatic expression of Nampt, Shp, and P53 $(\mathrm{p}<0.10)$ and a trend toward increased expression of $\operatorname{Fxr}(\mathrm{p}<0.10)$. Female CSE offspring in a fasted state exhibited no difference in hepatic mRNA expression for Sirt1 or the expression of the miRNA miR-34a.

Both male and female CSE offspring in a fed state exhibited increased mRNA expression of Sirt1. However, only fed male CSE offspring exhibited a trend toward increased Nampt mRNA expression while only fed female offspring exhibited increased Fxr and Shp mRNA expression. Female CSE offspring in a fasted state exhibited increased mRNA expression of several genes in the SIRT1 regulatory loop while male CSE offspring in a fasting state showed no significant difference in mRNA expression in the SIRT1 regulatory loop. It should be noted that there was no change in the hepatic expression of miR-34a, a well-established microRNA associated with the suppression of both protein and mRNA expression of SIRT1 and NAMPT in either fasted male or female offspring subjected to in utero cigarette smoke exposure. These data show that in a fasted state, any modulation of SIRT1 regulatory loop components as a result of in utero cigarette smoke exposure was independent of miR-34a. These data suggest that modulation of SIRT1 regulatory loop components may have been enacted via stimulation from outside of the primary regulatory loop.

It should be noted that for the hepatic expression of Sirt1 and Nampt, the directionality of mRNA expression did not always match that of their corresponding protein. For example, in fed male CSE offspring, SIRT1 protein expression was increased while mRNA was unchanged. Male CSE offspring in a fed state also exhibited no difference in NAMPT protein expression, while mRNA expression was increased. The only two instances where the mRNA and protein expression were matched was in fed female CSE offspring exhibited increased mRNA and protein expression of SIRT1 and fasted female CSE offspring exhibited no change in mRNA and protein expression of SIRT1. These results suggest that there may be a disconnect between 
transcription of mRNA and subsequent translation into protein, or there is a post-translational factor that is influencing the stability of proteins and their expression levels relative to their mRNA expression levels.

\section{In utero cigarette smoke exposure resulted in modulation of SIRT1-deacetylated}

\section{transcription factors and their downstream targets based on sex and fasting status}

Examining the effects of prenatal CSE on the hepatic expression of SIRT1-deacetylated transcription factors and their downstream targets revealed marked differences between weanling male and female offspring in a fed state. In Figure 19A, fed male offspring in a fed state exposed to in utero cigarette smoke have increased expression of Bsep and Cyp8b1 $(p<0.05)$ while Pgc$1 \alpha$ exhibited a trend toward increased expression $(p<0.1)$. In fed male offspring subjected to in utero cigarette smoke exposure also exhibited a decrease in Ppara expression $(p<0.05)$ and a trend toward decreased Srebp-1c $(p<0.10)$. No other changes in mRNA expression were detected in male offspring in a fed state. In Figure 20A, male CSE offspring in a fasted state exhibit increased hepatic expression of G6pase, Pepck, and Mcad $(\mathrm{p}<0.05)$ while exhibiting a trend toward increased Cyp7a1 expression and a trend toward decreased $\beta$-Klotho expression ( $p$ $<0.10)$. No other changes were detected in male CSE offspring in a fasted state.

Fed female CSE offspring in Figure 19B exhibited increased hepatic expression $(p<0.05)$ of fat metabolic genes (Mcad, Srebp-1c), glucose metabolic genes (Pgc-1a, Crtc2), and genes involved with FXR signaling (Ost- $\beta$, Bsep, Fgfr4, $\beta$-Klotho, Hnf4a, Lxr- $\alpha$, Lrh1, Cyp7a1). Fed female offspring subjected to in utero cigarette smoke also saw a trend toward increased Pepck and Ehhadh expression $(p<0.10)$. Female CSE offspring in a fed state exhibited no other changes in mRNA expression. As seen in Figure 20B, fasted female offspring subjected to in utero cigarette smoke exposure exhibited increased hepatic expression of genes $(p<0.05)$ in glucose metabolism (Pepck, Crtc2, Gapdh, and Pdk-4), fat metabolism (Ppara, Ehhadh, Srebp-1c, Fas), and FXR signaling (Bsep and Lrh1). Female CSE offspring in a fasted state also exhibited a trend toward increased hepatic expression of Pgc-1 $\alpha$, G6pase, and Ost $\beta(p<0.10)$. Female CSE offspring in a fasted state exhibited no other differences in mRNA expression. 


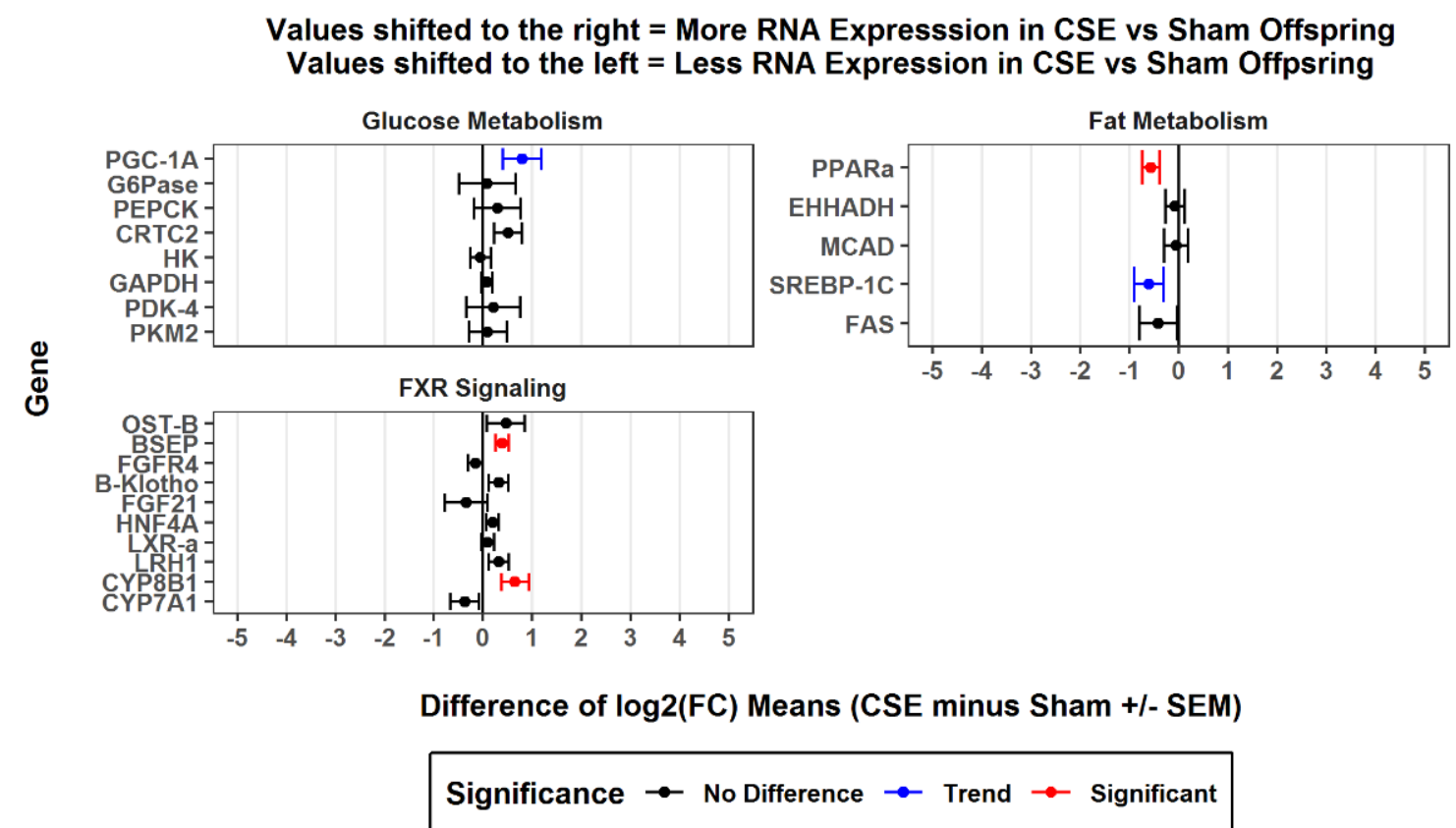

Figure 19 - Prenatal cigarette smoke exposure in fed offspring differentially modulated hepatic gene expression of SIRT1-related metabolic processes by sex - mRNA expression of genes was measured using the livers of offspring euthanized on postnatal day 19 in a fed state. A) Fed Male Offspring - The expression of Bsep and Cyp8b1 was significantly increased in fed male offspring in response to in utero cigarette smoke exposure, while Pgc-1a exhibited a trend toward increased expression. Fed male CSE offspring exhibited decreased expression of Ppara and exhibited a trend toward decreased Srebp-1c expression. Results are presented as the difference between the group means of log2 transformed data. Significant results had a $p$ value $<0.05$ while trends had a $p$-value $<0.1$ 


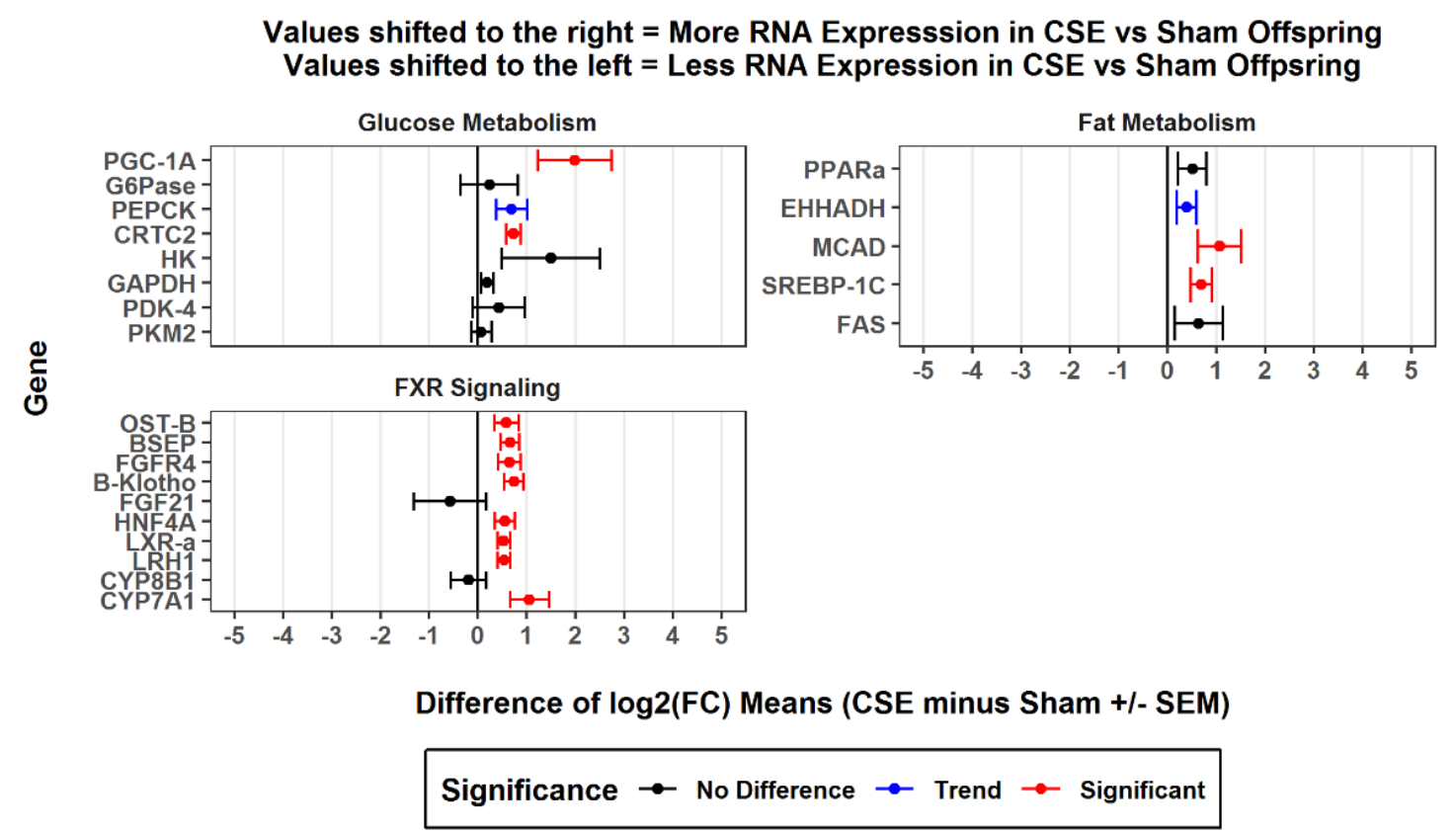

Figure 19 - Prenatal cigarette smoke exposure in fed offspring differentially modulated hepatic gene expression of SIRT1-related metabolic processes by sex - mRNA expression of genes was measured using the livers of offspring euthanized on postnatal day 19 in a fed state. B) Fed Female Offspring - In fed female offspring subjected to in utero cigarette smoke exposure, the expression of numerous genes in fat metabolism, FXR signaling, and gluconeogenic genes were significantly upregulated. Fed female CSE offspring also exhibited a trend toward increased expression of Ehhadh and Pepck. Results are presented as the difference between the group means of log2 transformed data. Significant results had a $p$-value $<0.05$ while trends had a $p$-value $<0.1$. 


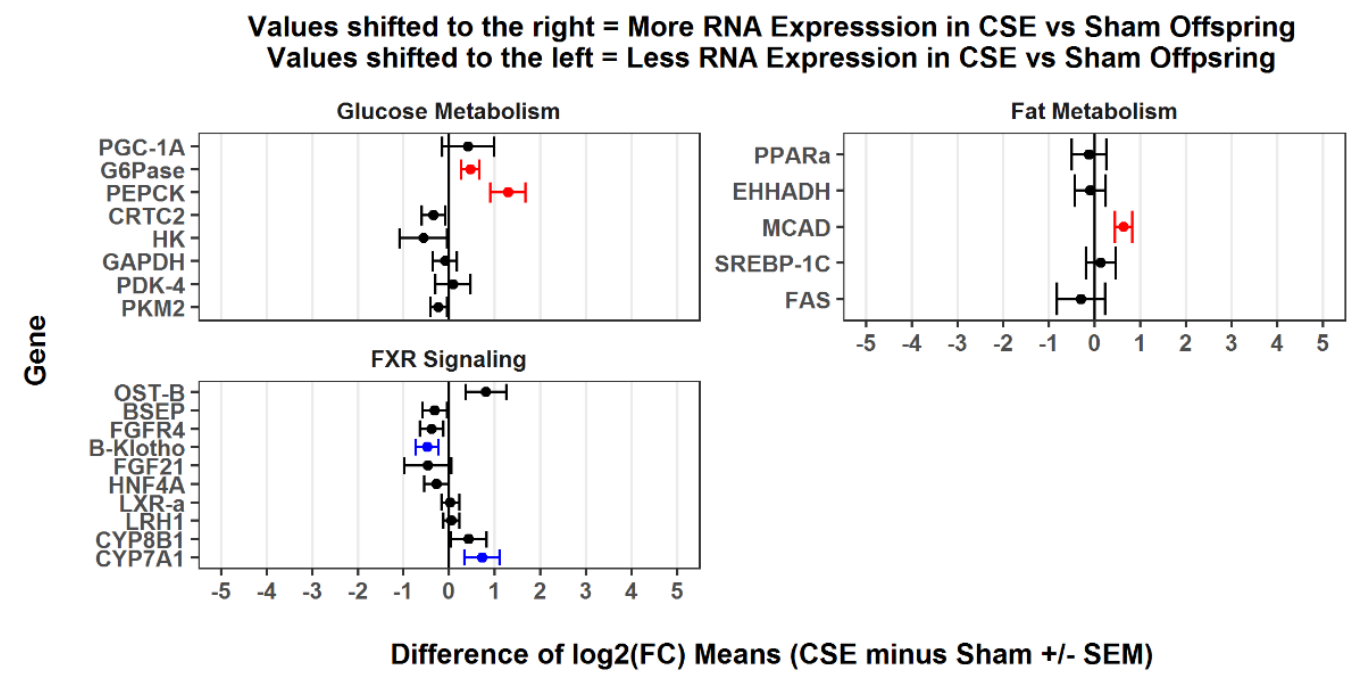

Significance $\rightarrow$ No Difference $\rightarrow$ Trend $\rightarrow$ Significant

Figure 20 - Prenatal cigarette smoke exposure in fasted female offspring exacerbated fasting response-induced hepatic gene expression - mRNA expression of genes was measured using the livers of offspring euthanized on postnatal day 19 in a fasted state. A) Fasted Male Offspring - The expression of gluconeogenic genes G6pase and Pepck in addition to the lipolytic gene Mcad were significantly increased in male offspring in response to in utero cigarette smoke exposure. Fasted male CSE offspring exhibited a trend toward increased Cyp7a1 expression and a trend toward decreased Sirt1, P53, and $\beta$-Klotho expression. Results are presented as the difference between the group means of log2 transformed data. Significant results had a $p$-value $<0.05$ while trends had a p-value $<0.1$. 


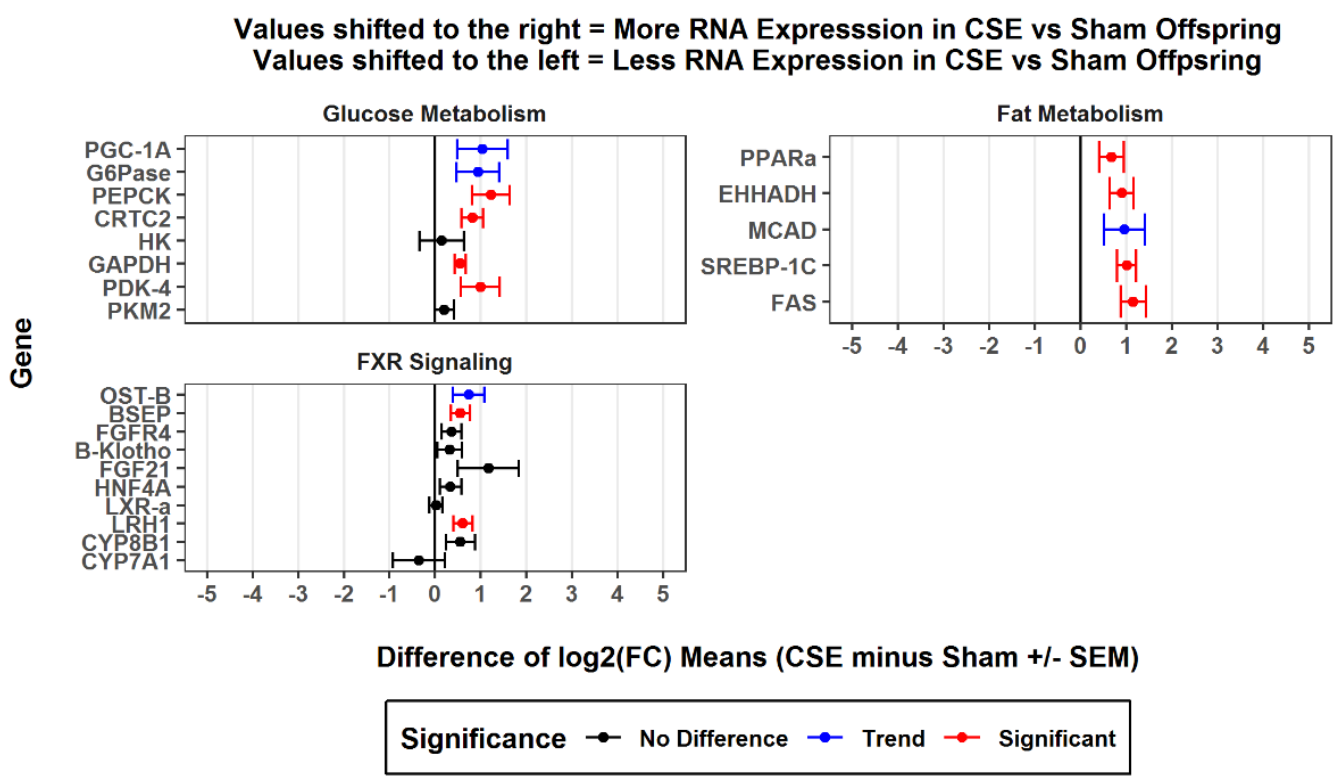

Figure 20 -Prenatal cigarette smoke exposure in fasted female offspring exacerbated fasting response-induced hepatic gene expression - mRNA expression of genes was measured using the livers of offspring euthanized on postnatal day 19 in a fasted state. B) Fasted Female Offspring - The expression of numerous genes in fat metabolism, FXR signaling, and glucose metabolism were significantly upregulated in fasted female offspring subjected to in utero cigarette smoke exposure. Fasted female CSE offspring also exhibited a trend toward increased expression of Pgc-1a, G6pase, Mcad, and Ost- $\beta$. Results are presented as the difference between the group means of log2 transformed data. Significant results had a pvalue $<0.05$ while trends had a $p$-value $<0.1$ 
It was evident when comparing the impact of in utero cigarette smoke exposure in male versus female offspring that mRNA expression of downstream SIRT1 target genes was starkly different. Overall, weaned female offspring in a fed state experienced a more extensive array of hepatic gene expression changes after being exposed to cigarette smoke in utero while male offspring did not appear to be altered much concerning hepatic gene expression. It seems that the female CSE offspring exhibited exacerbated fed and fasted responses. Male CSE offspring, however, seemed to not have an exacerbated fed response in a fed state and a mildly exacerbated fasted response in a fasted state relative to female CSE offspring. The sex-specific impact of exposure to in utero cigarette smoke is highly modulated by a fed or fasted state, especially in female CSE offspring.

\section{Discussion}

As we observed in Chapter III, offspring subjected to in utero cigarette smoke exhibited a low birth weight phenotype. After PD4, there was no longer an observable difference between prenatal CSE and Sham exposures, which was still evident by the time the offspring were weaned and then euthanized at 19 days of age. As also noted in Chapters II and III, our model of gestational only cigarette smoke exposure results in a well-defined phenotype of low birth weight followed by catch-up growth consistent with the Barker Hypothesis.

We observed that the decreased levels of serum glucose that were found in female CSE offspring 1.5 weeks old in Chapter III are no longer present in the weaned fed female CSE offspring from the current study. This finding suggests that the potential liver underdevelopment that may have occurred with female CSE offspring aged nine days was rectified by the time they reach the weaning stage in their life cycle. After weaning, the offspring subjected to prenatal cigarette smoke in utero appear normal concerning physical phenotype. This finding is in contrast to human studies, which show that adolescent children subjected to cigarette smoke in utero not only exhibit decrements in height-for-age [350, 351] but also an increased risk of obesity [352, 353]. A prior murine study conducted by our lab examining the impact of pre- and postnatal cigarette smoke exposure found that at PD21, CSE offspring did not exhibit altered serum glucose levels compared 
to Sham offspring [35]. This absence of serum glucose decrements is similar to the outcomes of the current present study. Another study from our lab found that at six months of age, offspring subjected to pre- and postnatal CSE did exhibit decreased serum glucose levels [40]. It seems that a lack of overt perturbations to physical phenotype shortly after weaning but which manifests into an altered phenotype later in adulthood in mice subjected to in utero cigarette smoke exposure is reasonable to conclude based on the evidence from both past and current studies from our lab. Additionally, this murine pattern to prenatal cigarette smoke exposure-induced phenotypic development may be a distinct feature from the phenotypic development observed in humans. These types of observations are critical to note when applying murine findings to humans and for developing potential health interventions.

We found in our study that male and female CSE offspring in a fed state exhibited decreased hepatic expression of NAMPT protein. However, in a fasted state, only male CSE offspring exhibited decreased NAMPT protein expression. It could be inferred from this that a decrement in $\mathrm{NAD}^{+}$and thus SIRT1 deacetylase activity would occur; however, the level of acetylated FKHR, a known target of SIRT1-induced deacetylation, remained unchanged in male CSE offspring in either a fed or fasted state and in female CSE offspring in a fed state. Male CSE offspring during a fasted state and female CSE offspring in a fed state exhibited increased expression of SIRT1 in conjunction with decreased expression of NAMPT. Due to these results, we currently cannot definitively determine how SIRT1 deacetylase activity was impacted by in utero cigarette smoke exposure in these offspring. One step that could have helped determine the impact of NAMPT expression on SIRT1 deacetylase activity is measuring the levels of NAD+. It is crucial to consider the overall effect of the $\mathrm{NAD}^{+}$levels on the resultant phenotypes observed in the current study since, in the context of aging, the cellular pool of $\mathrm{NAD}^{+}$naturally decays over time and that anything that accelerates the aging process could, in turn, exacerbate the diminishing of the NAD+ pool [354]. In the context of our experiment, the decrement in hepatic NAMPT expression in fed and fasted male CSE offspring and fed female CSE offspring could be an indicator of accelerated aging that will more readily manifest itself later in life in the form of metabolic disease. This process could also be exacerbated by the consumption of a high-fat diet, which has been reported to 
decrease levels of hepatic NAMPT [262]. This phenomenon may be particularly pertinent for male CSE offspring since they exhibited a decrement in NAMPT protein expression in either a fed or fasted state. This decrement that occurred in male CSE offspring regardless of fasting status may be a manifestation of increased susceptibility of male offspring to in utero cigarette smoke exposure. The data presented are novel findings that may help explain why the Barker Hypothesis predicts that male offspring are more susceptible to in utero perturbations.

A significant finding from the current present study was the apparent exacerbated fastinginduced hepatic mRNA expression in response to exposure in utero to cigarette smoke. This exacerbation was apparent in both male and female CSE offspring but to different degrees. Male CSE offspring during a fasted state exhibited more signs of an exacerbated fasting response with an increased hepatic expression of both G6pase and Pepck, two classically upregulated gluconeogenic enzymes during fasting. Fasted male CSE offspring also exhibited increased expression of Mcad, a lipolytic enzyme that is upregulated during a fasting response. Initially, these results seem counter to the fact that fasted male CSE offspring also exhibited decreased Sirt1 expression but also exhibited increased expression of SIRT1. However, fasted male CSE offspring exhibited decreased expression of NAMPT, so it is unclear how SIRT1 activity was being functionally impacted. It would seem that looking merely at protein and gene expression of SIRT1 and NAMPT would suggest a downregulation of SIRT1 activity, but there are numerous factors outside of expression levels that can impact SIRT1 including but not limited to post-translational modification [339], location within the cell [339], NAD+ levels[262], and interacting proteins that directly modulate SIRT1's catalytic activity [271, 272]. Overall, it appears that there is an exacerbation of the fasting response in fasted male offspring. However, it is not at the level of exacerbation as exhibited by fasted female CSE offspring.

Fasted weanling female CSE offspring have more genes being upregulated compared to fasted male CSE offspring in addition to increased lipolytic-related genes. Additionally, fasted female CSE offspring exhibited increased expression of Gapdh and decreased expression of Hexokinase. GAPDH, although an enzyme classically associated with glycolysis, is also an important enzyme needed for gluconeogenesis to occur effectively via deacetylation of GAPDH 
[355] and its gene expression is upregulated during the early stages of the fasting response in a murine model [356]. Lower levels of Hexokinase activity are critical during the fasting response since the downregulation of this enzyme allows glucose to leave the liver to maintain circulating blood glucose levels [194], and its hepatic protein expression was decreased in the current study in fasted female CSE offspring. Pdk-4, which inhibits glucose utilization by inhibiting the pyruvate dehydrogenase complex and is increased during fasting and starvation [357], was also increased in fasted female CSE offspring. Almost paradoxically, there was the presence of upregulation of lipogenic genes such as Srebp-1c and Fas in addition to lipolytic genes Ppara, Ehhadh, and a trend toward upregulation of Mcad. Lipogenic genes are typically upregulated following feeding and suppressed during fasting [358]. However, it is possible that lower expression or activationresistance of negative upstream regulators of Srebp-1c such as leptin to result in increased SREBP-1C-induced lipogenesis even when energetically inappropriate [359, 360]. Additionally, research suggests that cigarette smoking while pregnant reduced umbilical cord levels of leptin [361, 362]. It is possible that in our current model, in utero cigarette smoke exposure results in perturbing leptin signaling in offspring and would account for the induction of both lipogenic and lipolytic genes during fasting that we observed. Collectively, the data show that female CSE offspring in a fasted state exhibit an exacerbated fasting phenotype above what is found in a normal fasted state. Overall, we conclude that weaned offspring responded to in utero cigarette smoke exposure exhibit sex-specific exacerbation of the fasting response, and this could be a novel mechanism that contributes to sex-specific increases in the risk of metabolic disease later in life as predicted by the Barker Hypothesis.

Another significant finding from the current present study was the prenatal CSE-induced sex-specific responses in hepatic mRNA expression during a fed state. In male CSE offspring in a fed state, there were few changes to hepatic mRNA expression with the notable exceptions of decreased Ppara and increased Sirt1. There was a trend toward increased expression of Pgc-1a in addition to a trend toward decreased expression of Srebp-1c that would reflect increased gluconeogenic and decreased lipogenic activity, respectively, which is what would be expected during fasting with increased SIRT1 activity. It is not clear whether the changes in hepatic gene 
expression that fed male CSE offspring exhibited result in a functional impact on their metabolic phenotype.

Conversely, female CSE offspring in a fed state exhibited increased expression of Sirt1related genes as well as increased expression of gluconeogenic and lipolytic genes. Increased hepatic Sirt1 mRNA expression in female CSE offspring in a fed state along with the upregulation of Shp expression suggests that the SIRT1 regulatory loop was enhanced in the fed state. These results suggest that female CSE offspring developed a metabolic phenotype where the current energy status is not detected correctly. Additionally, these results indicate that female CSE offspring exhibit an inappropriately timed hepatic fasting response in a fed state. Additionally, in a fed state, female CSE offspring exhibit globally increased expression of various Fxr-related genes including Fxr itself. In particular, there is an increase in the expression of Fgfr 4 and $\beta$-Klotho which has been shown in the literature to enhance intestinal Fgf15 signaling [363]. The human analog of FGF15, FGF19, is essential in inducing glycogenesis after a meal [364]. Once glycogen stores are replenished, excess glucose is converted to fatty acids through de novo lipogenesis [358]. All this information together suggests that fed weanling female CSE offspring have their hepatic metabolism primed to store excess energy from food consumption more efficiently. The results presented here are a novel finding that may help explain why in Chapter II, female CSE offspring maintained on a high-fat diet gain weight so rapidly if the hepatic metabolic phenotype observed in the current present study is propagated into adulthood.

A novel finding from the current present study was the sex-specific prenatal CSE-induced modulation of acetylated proteins in fasted offspring. Male CSE offspring in a fasted state, despite the presence of increased hepatic SIRT1 protein expression, exhibited only a trend toward decreased acetylated lysine residues. This outcome may, in part, be due to male CSE offspring in a fasted state, also exhibited decreased hepatic NAMPT protein expression. If this decrease in NAMPT expression negatively impacted NAD+ levels, it could explain why there was no net effect on protein acetylation. In contrast, female CSE offspring in a fasted state exhibited decreased hepatic expression of both acetylated FKHR, a known deacetylation target of SIRT1, and acetylated lysine residues. These results in female CSE offspring suggest that in a fasted state, there is 
something that is influencing the levels of acetylated proteins that is independent of SIRT1 protein expression levels. Various factors that can influence SIRT1 deacetylase activity were mentioned earlier in the discussion, but another factor that should be considered in the context of our results is the cellular pool of Acetyl-CoA that is available. It is known that the levels of available acetyl-CoA influence the enzymatic activity of acetyltransferase enzymes, such as EP300 [365]. Lower bioavailability of acetyl-CoA can result in less acetylation of proteins and thus play a role in the trend toward decreased acetylated lysine residues in fasted male CSE offspring. Lower levels of available acetyl-CoA are a factor to consider as well in fasted female CSE offspring since they exhibited decreased expression of both acetylated FKHR and proteins with acetylated lysine residues without any changes in the protein expression of either SIRT1 or NAMPT. Even though the results reflect the possibility of increased SIRT1 activity in female CSE offspring in a fasted state, lower availability of acetyl-CoA could provide an alternative hypothesis as to the apparent exacerbated fasting response seen in female CSE offspring in a fasted state. This hypothesis is especially important to consider since acetylated FKHR, and acetylated lysine residues alone do not confirm that these changes in protein acetylation were due to strictly SIRT1 deacetylase activity. Lower acetyltransferase activity due to depleted pools of acetyl-CoA [365] also has the potential of being the cause of decreased protein acetylation seen in the current present study. We conclude that our results provide evidence that the greater exacerbation of the fasting response seen in female CSE offspring relative to male CSE offspring is driven in part by decreased protein acetylation. This decrease in protein acetylation in female offspring subjected to in utero cigarette smoke exposure provides further evidence of the need to examine sex-specific outcomes with regard to the Barker Hypothesis.

Future studies will examine siblings from our current study that have reached adulthood and were maintained on either a low-fat control diet or a high-fat diet in order to determine if the changes in hepatic expression of SIRT1 and related metabolic enzymes are seen in Chapters III and IV are correlated with predicted enhanced weight gain in response to high-fat diet feeding. Future studies will also examine if perturbations to the gut microbiome of offspring occur in response to prenatal cigarette smoke exposure and high-fat diet maintenance. We confirmed in 
our novel study that prenatal cigarette smoke exposure resulted in sexually dimorphic hepatic gene and protein expression alterations without overt hepatotoxicity. Sexually distinct responses to in utero cigarette smoke exposure exist post-weaning and before sexual maturity and are highly dependent on fasting status. Our study presents novel insights into the hepatic molecular phenotype induced by in utero exposure to cigarette smoke in a sex- and fasting state-dependent manner. 


\section{CHAPTER V: DISCUSSION}

The overarching goal of the dissertation work documented here was to characterize the age-specific as well as the sex-specific impact of in utero cigarette smoke exposure on offspring hepatic SIRT1-related metabolic function and how this fulfills the Barker Hypothesis-predicted perturbation of metabolic phenotypes in adulthood. Experiments in Chapter II aimed to characterize the sex-specific impact of prenatal cigarette smoke exposure in adult offspring consuming a high-fat diet on the manifested hepatic molecular phenotype, including hepatic protein expression of SIRT1 and related enzymes. The goal of the study in Chapter III was to determine the sex-specific impact of in utero cigarette smoke exposure on offspring hepatic molecular phenotype through hepatic SIRT1 and related gene and protein expression in the neonatal period. Chapter IV experiments were designed to determine how prenatal cigarette smoke exposure influences the hepatic molecular phenotype through hepatic SIRT1 and related gene and protein expression after weaning based on the sex of the offspring and current fasting status. Collectively

these experiments provide new insight into potential mechanistic pathways of how prenatal cigarette smoke exposure impacts the hepatic molecular phenotype of offspring at various critical life stages and provides new observations as to how the Barker Hypothesis results in a sex-specific impact of in utero cigarette smoke exposure.

\section{Low Birth Weight and Catchup Growth Phenotypes}

One of the significant outcomes of this dissertation work is the presence of a low birth weight phenotype with subsequent catch-up growth, as seen in Chapters II through IV. The manifestation of this phenotype in response to in utero cigarette smoke exposure agrees with the basic premise of the Barker Hypothesis, in which prenatal exposure to cigarette smoke results in perturbed offspring health outcomes [150]. However, comparing the results of this dissertation 
work with the literature illustrates that depending on the timing, duration, and severity of the exposure, the degree of low birth weight and whether subsequent catch-up growth occurs can vary. Also complicating this is the potential of maternal and paternal factors to modulate the magnitude of low birth weight and subsequent catch-up growth.

One retrospective cohort study found that when the BMI of mothers who smoked increased, the severity of the low birth weight phenotype in offspring decreased [366]. A Japanese cohort study found that the association between maternal smoking during pregnancy and low birth weight in offspring increased with maternal age [367]. Even if the low birth weight phenotype is not present, it is possible for the weight gain of offspring exposed in utero to cigarette smoke to be affected adversely. Another Japanese study found a positive dose-response relationship of smoking during pregnancy to the risk of rapid weight gain during infancy [368]. In terms of paternal factors, male mice that were exposed to CSC during puberty resulted in offspring that had a lowbirth-weight but still did not exhibit catch-up growth by six weeks after birth [369]. The effect of paternal exposure to CSC during puberty on catch-up growth was not present when maternal CSC exposure during pregnancy also occurred [369].

Another factor that could influence low birth weight and catch up growth outcomes is maternal glucocorticoid usage during pregnancy. Maternal glucocorticoid usage is of particular concern since glucocorticoid administration is utilized in managing the risk of preterm birth [370], which is a well-documented risk of prenatal cigarette smoke exposure [371-378]. It has been found that a single prenatal treatment with glucocorticoids is associated with low birth weight in term infants [379]. It has also been found that multiple courses of prenatal corticosteroid treatment also increases the risk of low birth weight [380, 381]. Even increased glucocorticoid levels independent of synthetic glucocorticoid administration can be sufficient to trigger similar results. One prospective longitudinal study that examined levels of placental corticotropin-releasing hormone $(\mathrm{pCRH})$ and maternal plasma cortisol found that increased pCRH exposure at gestational week 30 was associated with low birth weight and subsequent catch-up growth by either within the first 12 months or within 12 to 24 months [382]. It is clear that to fully characterize how prenatal cigarette smoke results in low birth weight with subsequent catchup growth, it is necessary to determine how 
a variety of both maternal and paternal factors could modulate the health outcomes of offspring subjected to in utero cigarette smoke exposure. Doing so has the potential to clarify the reason for some of the inconsistencies present in the literature regarding low birth weight and subsequent catchup growth of offspring that are induced by maternal smoking.

\section{Impact of Prenatal CSE on Development of Obesity and Fatty Liver in Adulthood}

A hypothesis of interest for this dissertation work was whether there were any indications of prenatal CSE in inducing a hepatic molecular phenotype that promotes the development of obesity and fatty liver disease. The early stage of non-alcoholic fatty liver disease (NAFLD) is the accumulation of lipids in the liver, which is often accompanied by obesity. In Chapter II, we found that adult male CSE offspring exhibited a trend toward increased weight gain when maintained on either a low-fat control diet or a high-fat diet. Additionally, we found that male offspring gained weight at a higher rate if fed a high-fat diet regardless of prenatal exposure. We also found that male offspring maintained on a high-fat diet exhibited elevated body fat accumulation regardless of prenatal exposure. We also observed that only male offspring maintained on a high-fat diet exhibited elevated liver triglycerides with no impact by in utero cigarette smoke exposure. It would seem that exposure to prenatal cigarette smoke did not enhance the rate of obesity or liver lipid accumulation in male offspring. However, the lack of an impact of in utero cigarette smoke exposure on obesity or fatty liver in male offspring could have been in part due to our choice of a low-fat diet, which contained approximately $5 \%$ fat, as the control to the high-fat diet. It is possible that had we chosen a control diet that had higher levels of fat, we could have observed prenatal CSE-induced obesity or fatty liver development. Additionally, we did not observe an exacerbation of the obesity- and fatty liver-inducing effects of a high-fat diet by in utero cigarette smoke exposure in male offspring. There are two possibilities for this outcome. First, there could be no impact of prenatal CSE on exacerbating the effects of high-fat diet-induced obesity or fatty liver. Secondly,

the 3-month long duration of the high-fat diet maintenance may have initially revealed some exacerbation by prenatal CSE but was overtaken by the continued maintenance on a high-fat diet. 
We plan to investigate this factor in currently ongoing studies. Overall, we found that prenatal CSE did not contribute significantly to the development of either obesity or fatty liver.

In female offspring subjected to in utero cigarette smoke exposure as described in Chapter II, we did observe an exacerbation of weight gain and body fat accumulation when maintained on a high-fat diet. No change was detected; however, in female CSE offspring, when maintained on a low-fat control diet. It is evident that in utero cigarette smoke exposure in female offspring was capable of acting with high-fat diet maintenance to exacerbate the development of obesity. However, none of the female offspring exhibited any sign of liver lipid accumulation. Not even maintenance on a high-fat diet resulted in any change in liver lipid levels. Given the apparent effect of prenatal CSE on exacerbating the effects of high-fat diet maintenance on developing obesity, it was surprising to see no impact on liver lipid accumulation. Although surprising to us, these outcomes in female offspring are consistent with the literature. The literature shows that females, in general, are more resistant to the acquisition of a NAFLD phenotype. Of note is that women no longer show this resistance to NALFD development when they are post-menopausal [383] and that a greater magnitude of estrogen-deficiency (i.e. premature menopause) exacerbates the severity of NAFLD-related fibrosis independent of age or any other metabolic disease [384]. That estrogen signaling could be mediating the severity of NAFLD development is also seen in a study in which ovariectomized mice developed more severe liver injury compared to sham-operated mice when fed a high-fat-high-cholesterol diet [385]. The same study found that the severity of the diet-induced liver injury was lessened when the ovariectomized mice were given estrogen treatment [385]. According to one study, this apparent resistance of females to NAFLD development may, in part, be due to being able to emphasize fatty acid transformation into ketone bodies rather than VLDLtriacylglycerol [386]. Overall, we conclude that the innate resistance of female offspring to developing fatty liver is present even when exposed in utero to cigarette smoke. This evidence supports the prediction of the Barker Hypothesis that female offspring are more resistant to the development of metabolic disease relative to male offspring as a result of prenatal cigarette smoke exposure. 
A facet of more advanced fatty liver disease is the progression of NAFLD to non-alcoholic steatohepatitis (NASH), in which the accumulation of lipids in the liver leads to the production of radical oxidation species (ROS). As a result, it is common to find antioxidant enzymes upregulated to counteract these effects. To detect whether there may be more advanced fatty liver disease pathology, we measured in Chapter II the hepatic protein levels of two antioxidant enzymes, superoxide dismutase (SOD2) and Catalase (CAT). We observed no effect in either male or female offspring on the hepatic protein expression of either of these enzymes by either prenatal exposure to cigarette smoke or maintenance on a high-fat diet. In conjunction with our other findings, these results indicate that the male offspring maintained on a high-fat diet have developed fatty liver disease. Conversely, female offspring on a high-fat diet did not develop any level of fatty liver disease, much less the later stages of steatohepatitis. Not even the exacerbation by prenatal CSE on weight gain and body fat accumulation in female offspring resulted in any progression into fatty liver disease. Whether the phenotypes that manifested in the current study would eventually progress given a more prolonged diet challenge remains unanswered. We conclude that given the current study design, female offspring subjected to prenatal cigarette smoke exposure exhibited sensitization toward increased high-fat diet-induced obesity and body fat accumulation without development of fatty liver disease.

\section{Impact of Prenatal CSE on Hepatic Metabolic Phenotype in Neonatal and Weaned Offspring and Contribution to Future Obesity and Fatty Liver Disease}

One question that we wanted to address with this work was whether prenatal cigarette smoke exposure perturbs the hepatic molecular phenotype at an earlier life stage before adulthood. This investigation was done to clarify how the perturbed sex-specific metabolic phenotypes observed in offspring in Chapter II may have developed as a result of prenatal cigarette smoke exposure. At 1.5 weeks of age, we found that male CSE offspring exhibited increased hepatic expression of SIRT1 and NAMPT mRNA and protein. This observation was in addition to increased

mRNA expression of PEPCK and CRTC2, two critical genes for gluconeogenesis. Although there was increased expression of SIRT1 and NAMPT for both mRNA and protein, there did not appear 
to be any noticeable impact on other components of the SIRT1 regulatory loop such as FXR, SHP, or P53. These results led us to conclude that something may be activating SIRT1-related pathways independent of the SIRT1-regulatory loop. Additionally, these results led us to conclude that there may be an appropriately timed fasting response in male offspring during the neonatal period. If these changes are related to the metabolic phenotype in adulthood, it is conceivable that the changes in hepatic molecular phenotype seen in male CSE offspring during the neonatal period could contribute to obesity and fatty liver development in adulthood.

In stark contrast, female CSE offspring exhibited a wide array of mRNA expression downregulation, including in glucose metabolism, fat metabolism, and FXR signaling. Additionally, female CSE offspring exhibited decreased mRNA expression of Nampt, Fxr, and P53. This latter result was surprising since those three genes in conjunction with Sirt1 participate in a regulatory loop where Sirt1, Nampt, Fxr, and Shp act in opposition to P53 and miR-34a. Due to the fact that this regulatory loop was globally downregulated in conjunction with several related gene pathways, we concluded that this was evidence of delayed functional liver ontogeny in female offspring exposed in utero to cigarette smoke. This delayed functional liver ontogeny was further supported by our observation of decreased serum glucose levels in female CSE offspring. However, these neonatal changes in the hepatic molecular phenotype occurred after the resolution of low birth weight and catch-up growth. We currently are unable to conclude if these changes in female CSE offspring are beneficial or detrimental to future development of obesity or fatty liver disease.

Another critical life stage to examine for potential contributions to future obesity and fatty liver disease was immediately after weaning. This allowed us to examine the impact of prenatal cigarette smoke exposure both in a fed and a fasted state. Male CSE offspring in a fed state exhibited decreased expression of NAMPT protein accompanied by increased Sirt1 mRNA expression. No other facets of the SIRT1-regulatory loop were affected in male CSE offspring in a fed state. The inconsistency between NAMPT protein expression and Sirt1 mRNA expression suggests that the SIRT1-regulatory loop was not being impacted, and something outside of this regulatory loop was affecting SIRT1 and NAMPT. Conversely, male CSE offspring in a fasted state still show decreased expression of NAMPT protein but with increased expression of SIRT1 protein 
and related gluconeogenic genes and Mcad, a typical upregulated lipolytic gene during a fasting response. However, male CSE offspring in a fasted state did not exhibit significant changes in the expression of other SIRT1-regulatory loop components, including miR-34a. It is evident that in light of the hepatic molecular phenotype present that male CSE offspring exhibited an exacerbated fasting response that appears to be independent of engagement of the SIRT1-regulatory loop. If the hepatic molecular phenotype observed in weaned male CSE offspring impacts future metabolic phenotype development, it is plausible that these changes may contribute to the development of obesity and fatty liver in adulthood.

It is without question that the most impacted offspring in this dissertation work were weaned females that had been subjected to in utero cigarette smoke exposure. In weaned female CSE offspring in a fed state, we observed increased protein expression of SIRT1 and decreased protein expression of NAMPT accompanied by increased mRNA expression of Sirt1, Fxr, and Shp. These changes appear to be independent of the SIRT1-regulatory loop since there was no change in the expression of P53 mRNA expression in addition to the decreased protein expression of NAMPT. We hypothesize that something was activating SIRT1-related pathways independently of the SIRT1-regulatory loop. Additionally, we observed that female CSE offspring in a fed state exhibited mRNA upregulation of FXR signaling as well as the gluconeogenic transcription factors Crtc2 and $P g c-1 \alpha$. We also observed mRNA upregulation of the lipogenic transcription factor Srebp-1c in female CSE offspring in a fed state. Overall, we believe that female CSE offspring in a fed state exhibited an exacerbated fed state with components of an inappropriately-timed fasting response. If these changes in hepatic molecular phenotype were to be propagated into adulthood, it is conceivable that female offspring subjected to in utero cigarette smoke exposure could experience enhanced development of obesity or fatty liver in adulthood.

Weaned female CSE offspring in a fasted state provided a surprising array of changes in the hepatic molecular phenotype, such as decreased protein expression of acetylated FKHR, a known SIRT1 deacetylation target that promotes gluconeogenesis, as well as decreased expression of hexokinase and acetylated-lysine protein residues. These results suggest but do not confirm the presence of enhanced SIRT1 deacetylation activity in weaned female CSE offspring in 
a fasted state. However, these changes were accompanied by mRNA upregulation of Nampt, Shp, and P53 without any change in mir-34a. It is evident that if SIRT1-related pathways and SIRT1 deacetylase activity were being upregulated in weaned female CSE offspring in a fasted state, it was occurring independently of the SIRT1-regulatory loop. It was evident that there was an exacerbation of the fasting response in mRNA expression in weaned female CSE offspring in a fasted state with the upregulation of gluconeogenic genes, lipolytic genes, as well as Pdk-4, which inhibits glucose utilization by inhibiting the pyruvate dehydrogenase complex and is increased during fasting and starvation [357]. What was surprising was the observed mRNA upregulation of lipogenic Sreb-1c and Fas in weaned female CSE offspring in a fasted state, which would seem counter-productive in a fasting response [358]. One potential explanation for this phenomenon would be a deficiency in leptin levels. Leptin is known to downregulate the expression and activity of Srebp-1c and thus downregulate the expression of Fas and that suppression of leptin levels or leptin receptor activity can result in inappropriately timed expression of Srebp-1c $[359,360]$. It has been shown that there are decreased levels of leptin in the umbilical cords of children subjected to in utero cigarette smoke exposure and that lower levels of leptin are associated with greater decrements in birth weight in response to intrauterine growth restriction. In light of this evidence, it is plausible that if the observed changes in hepatic molecular phenotype were to be propagated into adulthood that female CSE offspring would experience accelerated weight gain and body fat accumulation in adulthood, which matches what we observed in female CSE offspring maintained on a high-fat diet in Chapter II.

\section{Overall Strengths and Limitations}

One strength of this dissertation is the novelty in examining the hepatic molecular phenotype through measuring hepatic SIRT1 and related metabolic genes and proteins after being subjected to cigarette smoke exposure in utero in a strictly gestational model. As noted elsewhere in this work, our lab has previously demonstrated using a murine exposure model that cigarette smoke exposure in utero, in addition to postnatal exposure, results in the modulation of SIRT1 and related metabolic pathways (particularly with glucose metabolism). This dissertation, in addition to 
the past work of our lab, demonstrates that the livers of offspring subjected to in utero cigarette smoke exposure exhibit protein and gene expression changes related to SIRT1 and closely related metabolic targets. As far as we know, this dissertation is the first work in the literature to observe the hepatic molecular phenotype in adulthood as a result of a strictly prenatal cigarette smoke exposure as predicted by the Barker Hypothesis and explore how hepatic changes are potentially linked to these adulthood outcomes. Continuing this work will be vital in elucidating the molecular underpinning of how in utero cigarette smoke exposure translates into an increased risk of cardiometabolic disease in adulthood. Given how prenatal cigarette smoke exposure drives a low birth weight phenotype through intrauterine growth restriction $[28,29]$, this work could also be useful as a starting point for other in utero exposures to toxicants resulting in intrauterine growth restriction and the potential impact on health in adulthood. Once the underlying mechanism in our model is determined, this could provide new avenues for therapeutic interventions for children who are subjected to in utero cigarette smoke exposure.

Another strength of this study is the use of a whole-body inhalation exposure apparatus to expose pregnant mice to cigarette smoke. There are numerous studies that examine prenatal effects of cigarette smoke constituents other than nicotine in isolation on low birth weight including carbon monoxide [280, 281], benzo(a)pyrene [89], volatile organic compounds [94] including specifically formaldehyde [95] and benzene [96], polycyclic aromatic hydrocarbons [87, 88], and heavy metals like cadmium [282-285], but a significant disadvantage to this type of approach is that it undoubtedly fails to capture the complex interactive effects that each cigarette smoke component has with the others. Another disadvantage to exposing with individual components is that it involves either injection or oral ingestion, routes of exposure that have very different toxicological consequences compared to inhalation. Utilizing a cigarette smoking apparatus that exposes animals to both mainstream and sidestream smoke via whole-body inhalation provides a more realistic exposure scenario mimicking mothers who smoke while pregnant. Additionally, our model utilizes Marlboro Red cigarettes ${ }^{\mathrm{TM}}$ instead of a reference cigarette in part due to the presence of additives typically found in commercial cigarettes that are not present in reference cigarettes [387]. At the time of the development of this model, Marlboro Red ${ }^{\mathrm{TM}}$ cigarettes were the most commonly 
smoked cigarette [41]. This figure includes women aged 18-25, which was also the age group most likely to smoke at any time during pregnancy during this time [41]. This choice in cigarette was made to simulate a scenario of active maternal smoking while pregnant as accurately as possible. In our model, a whole-body exposure was used instead of a nose-cone based exposure mainly due to the usage of animal restraint necessary to ensure that the nose cone delivering cigarette smoke stays in place throughout the exposure. Given that restraining animals results in increased serum levels of glucocorticoids [388], in order to avoid the potential confounding effects of elevated glucocorticoid levels on postnatal health outcomes in the offspring, our model utilizes a whole-body inhalation exposure approach. A whole-body inhalation-based model has the added benefit of allowing the efficient exposure of multiple mice simultaneously while keeping them housed in their cage, which also helps minimize handling stress.

This dissertation work is novel in that it characterizes the hepatic molecular phenotype in response to prenatal cigarette smoke exposure at multiple key life stages. Although the Barker Hypothesis predicts that metabolic disease as a result of in utero exposure to cigarette smoke manifests itself in adulthood, it leaves unanswered if any disease occurs earlier, such as during the neonatal period or adolescence before sexual maturity. It also does not address if the changes that result in metabolic disease in adulthood are present at an early age or if the hepatic molecular phenotype dynamically shifts over the lifetime of the individual that is exposed in utero to cigarette smoke. This dissertation work addresses these points by characterizing the hepatic molecular phenotype not only in adulthood after a diet challenge, but also during the neonatal period and just after weaning. By examining prenatal cigarette smoke-exposed offspring during the neonatal period, at 1.5 weeks of age, we were able to examine offspring at a time when catch-up growth has occurred, and low birth weight is no longer evident. This examination provided insight as to the hepatic molecular phenotypes that are associated with low birth weight and subsequent catch-up growth. By examining post-weaned offspring, we were able to characterize the hepatic molecular phenotype in prenatal cigarette smoke-exposed offspring at a time before full sexual maturity as well as in a fed versus a fasted state. Examining in a fed versus a fasted state was especially crucial as it allowed us to answer the questions of whether offspring exhibited perturbed hepatic 
fed or fasted responses, gaining insight into the potential factors driving metabolic disease pathology in adulthood. These facets of our work bring important new findings to the literature since no one else has attempted to characterize the hepatic molecular phenotype at multiple critical life stages after being exposed in utero to cigarette smoke and how they relate to the manifestation of the Barker Hypothesis in adulthood.

What was especially novel about this work was the discovery that even at an early age, female offspring subjected to cigarette smoke in utero exhibited a greater number of perturbations in the hepatic molecular phenotype compared to male offspring. Additionally, female CSE offspring maintained on a high-fat diet experienced greater weight gain that was not present in male CSE offspring maintained on a high-fat diet. This result was surprising given that the Barker Hypothesis predicts that male offspring are more impacted by adverse in utero events compared to female offspring. However, because these female offspring after weaning appeared otherwise phenotypically normal, we cannot conclude whether these changes in the hepatic molecular phenotype are ultimately beneficial or detrimental to metabolic health in adulthood. Our findings reveal an urgent need to examine more closely at multiple life stages the sex-specific impact of prenatal cigarette smoke exposure on the hepatic molecular phenotype and any subsequent impact on developing metabolic disease.

With the current dissertation work, we were able to characterize a large number of gene expression changes in prenatal cigarette smoke exposed-offspring at various life stages before the induction of any metabolic disease pathology in adulthood. However, from the results of our study it is unclear whether the prenatal CSE-induced changes we observed do, in fact, lead to a fatty liver phenotype. Although adult male offspring maintained on a high-fat diet did develop obesity and fatty liver, there was no significant impact on these measures based on prenatal CSE. Additionally, female CSE offspring maintained on a high-fat diet did exhibit increased weight gain and body fat accumulation, but there there was no effect of prenatal CSE alone. Female offspring also did not develop fatty liver due to either high-fat diet or prenatal cigarette smoke exposure. We cannot definitively conclude whether prenatal cigarette smoke exposure, either alone or in conjunction with maintenance on a high-fat diet, contributes to fatty liver disease pathology in either sex. 
A factor that was not accounted for was observing prenatal CSE-induced low birth weight and subsequent catchup growth with respect to the sex of the offspring. Recording the sex of individual offspring from birth to weaning was not done due to the technical difficulty of individually tracking individual pups in a litter at such a young age as well as the difficulty in accurately sexing pups at a young age. Had we accomplished these tasks, it would have been possible to associate the severity of low birth weight with the hepatic molecular phenotype at various life stages and development of metabolic disease in adulthood. Additionally, we could have identified if there were offspring that responded strongly to prenatal cigarette smoke exposure versus offspring that responded weakly to prenatal cigarette smoke exposure. Identifying these "high-responders" versus "low-responders" would have provided another factor that could have been controlled for to refine further how the hepatic molecular phenotype is perturbed due to prenatal CSE and how this is linked to the development of metabolic disease. One downside to this approach is that it would likely require a larger sample size of offspring in experiments that would be more costly and timeconsuming. However, this is a necessary step to fully unravel the mechanism that drives the metabolic manifestations of the Barker Hypothesis.

One limitation of this dissertation lies in the duration of the diet challenge for the offspring in Chapter II. Although we did not observe any significant effects of prenatal CSE alone on weight gain, body fat accumulation, or fatty liver development, prenatal CSE could have exacerbated the effects of a high-fat diet at an earlier point in the diet challenge, and we did not observe it. Under this scenario, it is possible that the effects of long-term maintenance on a high-fat diet eventually obfuscated any initial effects that prenatal CSE may have contributed to the metabolic phenotype observed in offspring. Due to this potentially confounding factor, we cannot definitively conclude that all effects seen in the offspring in Chapter II in response to prenatal cigarette smoke exposure were not influenced by the prolonged duration of the diet challenge. In future studies we plan to examine adult offspring exposed in utero to cigarette smoke earlier in adulthood and shorten the duration of the diet challenge to ensure the potential confounding effects of long-term high-fat diet feeding do not exert undue influence on metabolic phenotype. 
Another limitation of our work was the choice of a low-fat diet as the control for the high-fat diet during the diet challenge in Chapter II. This factor is an essential consideration since the effects of high-fat diet maintenance may have been exacerbated by the low-fat content of the control diet (approximately $5 \%$ and $19 \%$ fat content, respectively). One way we could have helped account for this is by examining the hepatic molecular phenotype before the initiation of the diet challenge. Doing this may have provided additional context as to how prenatal cigarette smoke exposure affected the baseline hepatic molecular phenotype and how it is modulated by maintenance on either a high-fat or low-fat diet. In ongoing studies, we are examining adult siblings of the offspring from Chapters III and IV at a point before a diet challenge to address this limitation from Chapter II.

This ties into another limitation from our study; the fact that the adult offspring in Chapter II are not siblings of the offspring in Chapters III and IV. This factor is a significant limitation because although the prenatal cigarette smoke exposure parameters of the offspring in Chapters II versus Chapters III and IV were similar, they are not precisely the same. Although we were confident that the prenatal exposures were similar enough that it was reasonable to relate the changes seen in Chapter II to those in Chapters III and IV, we admit that there may be some other underlying difference between the two cohorts that confounded this comparison. This limitation is being addressed by currently in-progress studies examining adult siblings of the offspring from Chapters III and IV.

A factor that was not accounted for was the presence of mismatched levels of protein, and their corresponding mRNA levels within the same groups. For example, in Chapter III, neonatal female CSE offspring exhibited decreased hepatic expression of Sirt1, Nampt, Pepck, and Hk without any change in the corresponding protein levels. In other instances, the mRNA and protein expression matched each other such as the neonatal male CSE offspring exhibiting increased SIRT1 and NAMPT expression along with their corresponding mRNA in Chapter III. It is known that protein levels can be influenced independently from mRNA levels, such as through posttranslational modifications that can result in increased protein stability or promote proteasomal degradation, and SIRT1 is no exception to these naturally-occurring processes [339]. Additionally, 
it has been shown that levels of mRNA can be influenced by micro-RNAs, such as miR-34a being responsible for suppressing the expression of Sirt1 and Nampt, along with the corresponding proteins [262]. We attempted to look at the potential effects of miR-34a to modulate levels of Sirt1 and Nampt expression in fasted offspring in Chapter IV but found no difference in miR-34a levels despite changes in fasted male offspring exhibiting increased levels of SIRT1 and decreased levels of NAMPT while fasted female CSE offspring exhibited increased levels of NAMPT. These results suggest that levels of SIRT1, NAMPT, and their corresponding proteins were not subjected to modulation by CSE via a miR-34a-related pathway. We conclude from these data that the mismatched levels of SIRT1, NAMPT, and their corresponding mRNA is not due to modulation of the central SIRT1 regulatory pathway that includes miR-34a as a central component. In future studies, it is necessary to look at other pathways that interact with SIRT1, such as the mTORC pathways or mechanism involving post-translational modifications other than acetylation.

Tied into the previous limitation, we did not measure levels of either $\mathrm{NADH} / \mathrm{NAD}^{+}$or ATP/AMP. Measuring these would have provided valuable information as to the current cellular stores of energy in offspring subjected to in utero exposure to cigarette smoke. We observed that, for weaned female offspring in particular, there were signs of an exacerbated hepatic fasting response with respect to mRNA expression in response to prenatal cigarette smoke exposure. One possible explanation for this exacerbated fasting response in weaned female CSE offspring could have been that current cellular energy stores were being improperly sensed. If improper sensing of current cellular energy stores was occurring, this would provide novel insight into why the hepatic molecular phenotype is altered and would provide a potential mechanism to investigate further. Additionally, measuring levels of $\mathrm{NAD}^{+}$could have helped clarify how SIRT1 deacetylase activity was being impacted. In instances such as the weaned female CSE offspring in a fasted state exhibiting decreased hepatic protein expression of acetylated FKHR and acetylated lysine residues without a change in the protein expression of either SIRT1 or NAMPT, knowing the levels of NAD+ present could have allowed us to conclude better if this change in protein acetylation was due to a change in SIRT1 deacetylation activity. 
Another factor that we did not examine is the impact of cigarette smoke exposure on the quality of milk from the exposed dams. It is noted in the literature that smoking negatively impacts breastfeeding in a variety of ways, including but not limited to lower milk production [389], earlier weaning [389, 390], lower duration of breastfeeding [391], lower-fat concentration [389, 392, 393], altered breast milk lipid profile [394], lower cholesterol [395], lower protein [392, 395], heavy metal contamination [396-398], and lower antioxidative properties [399]. Additionally, smoking effectively cancels out the protective effects of breastfeeding on the risk of sudden infant death syndrome [400] and respiratory disorders [401]. Although we ceased exposure of pregnant dams to cigarette smoke at GD19, pups born to exposed dams could be adversely affected through the dams' milk for as long as cigarette smoke constituents and metabolites were still present within the dams. In future studies, it may be beneficial to conduct an experiment to determine what impact breast milk from cigarette exposure has on perinatal outcomes through a cross-fostering experiment. This experiment would allow for separation of the impact of in utero exposure to cigarette smoke from any confounding effect from altered milk quality or nursing behaviors.

\section{Future Directions}

One of the future studies that can be done to elucidate further how in utero cigarette smoke exposure modulates cardiometabolic perturbation in offspring would be via a systems biology approach that looks for associations with other body systems in exposed offspring. For example, examining changes in the brain, particularly in the hypothalamus, could be especially illuminating. It has been shown that in utero cigarette smoke exposure perturbs neurological development [402, 403] and given the importance of specific structures of the brain in controlling body-wide metabolism it would be prudent to interrogate if changes occur in these metabolic regulatory centers in response to prenatal cigarette smoke and if so, if these changes are related to the hepatic changes we have observed in our murine model. As mentioned previously, sex-specific secretion patterns of growth hormone are vital in determining the metabolic phenotype of the liver, and that perturbation of these secretion patterns can modulate the sex-specific imprinting of hepatic metabolic enzyme expression [242-244]. If changes to the growth hormone secretion pathway are 
apparent and associated with some of the hepatic changes we observed in our murine model, this could provide valuable insight into the hepatic and cardiometabolic pathology of the Barker Hypothesis as well as a potential avenue for therapeutic interventions of individuals affected by in utero cigarette smoke exposure.

A potential future study could further examine the SIRT1 regulatory pathway that includes miR-34a to confirm the importance of this pathway. Such an approach could include transgenic mice that would allow at-will perturbation of Sirt1 or miR-34a expression. An alternative approach to also test this could be the treatment of offspring with a SIRT1 promoter such as resveratrol or a SIRT1 inhibitor. Another way that could indirectly test the importance of the SIRT1 regulatory pathway would be to supplement the $\mathrm{NAD}^{+}$pool using nicotinamide mononucleotide (NMN), a precursor molecule for $\mathrm{NAD}^{+}$. Since SIRT1's deacetylase activity is dependent on $\mathrm{NAD}^{+}$as a cofactor, scenarios that affect levels of $\mathrm{NAD}^{+}$can have a net result of decreasing SIRT1 activity. Examples of this include $\mathrm{NAD}^{+}$consumers such as PARP1 increasing their activity [255] or the activity of NAMPT decreasing [262] result in decreased SIRT1 activity. Manipulating the NAD+ pool in addition to quantifying base levels of $\mathrm{NAD}^{+}$without supplementation could also help in determining whether the activity of SIRT1 is being modulated in our murine prenatal CSE model and thus contributing to the observations detected in the study.

Given the alarming rise in the rate of electronic cigarette and vaporizer use [4], especially in teenagers [5], it would be prudent to examine if similar changes we observed in this dissertation work were also present as a result in utero exposure to e-cigarette- or vaporizer-produced aerosols. Even if one only considered the effects of nicotine and none of the other components of e-cigarette solutions, there is an abundance of evidence that prenatal exposure to nicotine alone has numerous adverse effects, such as lower fetal body weight in addition to decreased fetal brain weight, loss of brain cells, and histological abnormalities in the brain [71]. These findings are not surprising given that nicotine acts as a vasoconstrictor, which limits blood flow from the mother to the placenta, thereby inducing intrauterine growth restriction [150]. Even if the resultant phenotype in e-cigarette/vaporizer aerosol-exposed offspring is not as severe as that induced by cigarettes, the upward trajectory of e-cigarette/vaporizer use indicates the possibility of a large number of 
individuals being affected, especially given the perception among some of the public that ecigarettes are inherently less harmful than cigarettes [404]. The implications of this on prenatal exposure to nicotine are potentially dire if expectant mothers who find themselves unable to wean off cigarettes instead switch to e-cigarettes due to perceived lower risk to health, which would still result in a significant number of fetuses being exposed to nicotine in utero. We are currently planning studies that will adapt our current murine in utero cigarette smoke exposure to utilize ecigarettes in order to characterize exposed offspring in the same manner we have in this dissertation work.

\section{Summary and Conclusions}

The unifying goal of the dissertation work described herein was to provide novel characterization the impact of in utero cigarette smoke exposure on the murine hepatic molecular phenotype via the expression of SIRT1-related gene and proteins at several life stages in conjunction with sex-specific altered metabolic phenotypes during adulthood as modulated by maintenance on a high-fat diet. This work was necessary to provide further evidence for how an in utero toxicant exposure results in a perturbed metabolic phenotype during adulthood, as predicted by the Barker Hypothesis. The study described in Chapter II of this dissertation provided initial support of how a gestation-only exposure to cigarette smoke-induced low birth weight with catch-up growth as well as other sex-specific metabolic, behavioral, and hepatic molecular phenotype changes in adulthood and how these phenotypic outcomes are modulated by the fat content of the diet the adult offspring consumed. Chapter II helped support the Barker Hypothesis by showing that gestational exposure to prenatal cigarette smoke exposure resulted in perturbed hepatic molecular phenotype in a sex-specific manner. These initial experiments inspired us to examine the hepatic molecular phenotype via SIRT1-related hepatic gene and protein expression in the liver at a young age shortly after birth in Chapter III. The findings in Chapter III suggest that, even as early as 1.5 weeks of age, the hepatic expression of SIRT1-related genes and proteins was perturbed in a sex-specific manner. These results suggest that future studies need to examine the sexual differentiation processes that occur perinatally. Chapter IV took the findings in Chapters 
II and III further by examining hepatic molecular phenotype through SIRT1-related gene and protein expression changes in response to in utero cigarette smoke exposure in weaned offspring in either a fed or a fasted state. Once again, there were apparent sex-specific perturbations in SIRT1related hepatic gene and protein expression with additional modulation based on fasting status. Offspring subjected to in utero cigarette smoke exposure, especially female offspring, have abnormal fed and fasted responses, but exactly how these particular changes are related to the altered metabolic phenotypes during adulthood that we observed in Chapter II will be the subject of future studies. The findings of this dissertation work have provided the initial characterizing steps necessary to more thoroughly investigate and probe the underlying mechanistic pathway that facilitates the adulthood metabolic manifestations of in utero cigarette smoke exposure as predicted by the Barker Hypothesis. 


\section{REFERENCES}

[1] A. Jamal, E. Phillips, A.S. Gentzke, D.M. Homa, S.D. Babb, B.A. King, L.J. Neff, Current Cigarette Smoking Among Adults - United States, 2016, MMWR Morb Mortal Wkly Rep 67(2) (2018) 53-59.

[2] A.S. Gentzke, M. Creamer, K.A. Cullen, B.K. Ambrose, G. Willis, A. Jamal, B.A. King, Vital signs: tobacco product use among middle and high school students-United States, 2011-2018, Morbidity and Mortality Weekly Report 68(6) (2019) 157.

[3] M.R. Creamer, T.W. Wang, S. Babb, K.A. Cullen, H. Day, G. Willis, A. Jamal, L. Neff, Tobacco product use and cessation indicators among adults_United States, 2018, Morbidity and Mortality Weekly Report 68(45) (2019) 1013.

[4] H. Dai, A.M. Leventhal, Prevalence of e-Cigarette Use Among Adults in the United States, 20142018, JAMA (2019).

[5] K.A. Cullen, B.K. Ambrose, A.S. Gentzke, B.J. Apelberg, A. Jamal, B.A. King, Notes from the field: Use of electronic cigarettes and any tobacco product among middle and high school students-United States, 2011-2018, Morbidity and Mortality Weekly Report 67(45) (2018) 1276. [6] P. National Center for Chronic Disease, S. Health Promotion Office on, Health, Reports of the Surgeon General, The Health Consequences of Smoking-50 Years of Progress: A Report of the Surgeon General, Centers for Disease Control and Prevention (US), Atlanta (GA), 2014.

[7] J. Ma, R.L. Siegel, E.J. Jacobs, A. Jemal, Smoking-attributable Mortality by State in 2014, U.S, Am J Prev Med 54(5) (2018) 661-670.

[8] M.B. Reitsma, N. Fullman, M. Ng, J.S. Salama, A. Abajobir, K.H. Abate, C. Abbafati, S.F. Abera, B. Abraham, G.Y. Abyu, Smoking prevalence and attributable disease burden in 195 countries and territories, 1990-2015: a systematic analysis from the Global Burden of Disease Study 2015, The Lancet 389(10082) (2017) 1885-1906.

[9] E. Gakidou, A. Afshin, A.A. Abajobir, K.H. Abate, C. Abbafati, K.M. Abbas, F. Abd-Allah, A.M. Abdulle, S.F. Abera, V. Aboyans, Global, regional, and national comparative risk assessment of 84 behavioural, environmental and occupational, and metabolic risks or clusters of risks, 1990-2016: 
a systematic analysis for the Global Burden of Disease Study 2016, The Lancet 390(10100) (2017) 1345-1422.

[10] C. Willi, P. Bodenmann, W.A. Ghali, P.D. Faris, J. Cornuz, Active smoking and the risk of type 2 diabetes: a systematic review and meta-analysis, JAMA 298(22) (2007) 2654-64.

[11] A. Pan, Y. Wang, M. Talaei, F.B. Hu, T. Wu, Relation of active, passive, and quitting smoking with incident type 2 diabetes: a systematic review and meta-analysis, The lancet Diabetes \& endocrinology 3(12) (2015) 958-967.

[12] S. Akter, A. Goto, T. Mizoue, Smoking and the risk of type 2 diabetes in Japan: a systematic review and meta-analysis, Journal of epidemiology $27(12)$ (2017) 553-561.

[13] S.H. Jee, A.W. Foong, N.W. Hur, J.M. Samet, Smoking and risk for diabetes incidence and mortality in Korean men and women, Diabetes care 33(12) (2010) 2567-2572.

[14] C.f.D. Control, Prevention, National diabetes statistics report, 2017, Atlanta, GA: Centers for Disease Control and Prevention, US Department of Health and Human Services (2017).

[15] G. Anifandis, T. Bounartzi, C.I. Messini, K. Dafopoulos, S. Sotiriou, I.E. Messinis, The impact of cigarette smoking and alcohol consumption on sperm parameters and sperm DNA fragmentation (SDF) measured by Halosperm((R)), Arch Gynecol Obstet 290(4) (2014) 777-82.

[16] H. Asare-Anane, S.B. Bannison, E.K. Ofori, R.O. Ateko, A.T. Bawah, S.D. Amanquah, S.Y. Oppong, B.B. Gandau, J.B. Ziem, Tobacco smoking is associated with decreased semen quality, Reprod Health 13(1) (2016) 90.

[17] A.K. Wesselink, E.E. Hatch, K.J. Rothman, E.M. Mikkelsen, A. Aschengrau, L.A. Wise, Prospective study of cigarette smoking and fecundability, Human Reproduction 34(3) (2018) 558567.

[18] A. Hyland, K.M. Piazza, K.M. Hovey, J.K. Ockene, C.A. Andrews, C. Rivard, J. WactawskiWende, Associations of lifetime active and passive smoking with spontaneous abortion, stillbirth and tubal ectopic pregnancy: a cross-sectional analysis of historical data from the Women's Health Initiative, Tob Control 24(4) (2015) 328-35. 
[19] B.L. Pineles, E. Park, J.M. Samet, Systematic review and meta-analysis of miscarriage and maternal exposure to tobacco smoke during pregnancy, American journal of epidemiology 179(7) (2014) 807-823.

[20] B.L. Pineles, S. Hsu, E. Park, J.M. Samet, Systematic review and meta-analyses of perinatal death and maternal exposure to tobacco smoke during pregnancy, American journal of epidemiology 184(2) (2016) 87-97.

[21] Y.A.A. Mindjah, F. Essiben, P. Foumane, J.S. Dohbit, E.T. Mboudou, Risk factors for ectopic pregnancy in a population of Cameroonian women: A case-control study, PloS one 13(12) (2018) e0207699.

[22] X. Xu, E.E. Bishop, S.M. Kennedy, S.A. Simpson, T.F. Pechacek, Annual healthcare spending attributable to cigarette smoking: an update, Am J Prev Med 48(3) (2015) 326-33.

[23] C.L. Baker, Y. Ding, C.P. Ferrufino, S. Kowal, J. Tan, P. Subedi, A cost-benefit analysis of smoking cessation prescription coverage from a US payer perspective, Clinicoecon Outcomes Res 10 (2018) 359-370.

[24] V. Gallo, D. Neasham, L. Airoldi, P. Ferrari, M. Jenab, P. Boffetta, K. Overvad, A. Tjonneland, F. Clavel-Chapelon, H. Boeing, V. Pala, D. Palli, S. Panico, R. Tumino, L. Arriola, E. Lund, B. Bueno-De-Mesquita, P.H. Peeters, O. Melander, G. Hallmans, E. Riboli, R. Saracci, P. Vineis, Second-hand smoke, cotinine levels, and risk of circulatory mortality in a large cohort study of never-smokers, Epidemiology 21(2) (2010) 207-14.

[25] J. Kulczycki, R.M. Olischer, Z. Osuch, [Cerebrospinal fluid cytological observations made in the course of meningeal, blastamatous processes], Psychiatr Neurol Med Psychol (Leipz) 26(11) (1974) 676-84.

[26] F. Fischer, A. Kraemer, Meta-analysis of the association between second-hand smoke exposure and ischaemic heart diseases, COPD and stroke, BMC Public Health 15 (2015) 1202.

[27] S. Yang, L. Xu, Y. He, C. Jiang, Y. Jin, K.K. Cheng, W. Zhang, T.H. Lam, Childhood secondhand smoke exposure and pregnancy loss in never smokers: the Guangzhou Biobank Cohort Study, Tobacco control 26(6) (2017) 697-702. 
[28] J. Dejmek, I. Solansk y, K. Podrazilova, R.J. Sram, The exposure of nonsmoking and smoking mothers to environmental tobacco smoke during different gestational phases and fetal growth, Environmental health perspectives 110(6) (2002) 601-6.

[29] M.S. Kramer, Determinants of low birth weight: methodological assessment and meta-analysis, Bull World Health Organ 65(5) (1987) 663-737.

[30] The Health Consequences of Smoking - 50 Years of Progress, in: U.D.o.H.a.H. Services (Ed.) 2014.

[31] A. Hackshaw, C. Rodeck, S. Boniface, Maternal smoking in pregnancy and birth defects: a systematic review based on 173687 malformed cases and 11.7 million controls, Hum Reprod Update 17(5) (2011) 589-604.

[32] K. Zhang, X. Wang, Maternal smoking and increased risk of sudden infant death syndrome: a meta-analysis, Leg Med (Tokyo) 15(3) (2013) 115-21.

[33] P.A. Fried, D.S. James, B. Watkinson, Growth and pubertal milestones during adolescence in offspring prenatally exposed to cigarettes and marihuana, Neurotoxicology and teratology 23(5) (2001) 431-6.

[34] A. Ernst, S. Kristensen, G. Toft, A. Thulstrup, L. Håkonsen, S. Olsen, C. Ramlau-Hansen, Maternal smoking during pregnancy and reproductive health of daughters: a follow-up study spanning two decades, Human reproduction 27(12) (2012) 3593-3600.

[35] L. Canales, J. Chen, E. Kelty, S. Musah, C. Webb, M.M. Pisano, R.E. Neal, Developmental cigarette smoke exposure: liver proteome profile alterations in low birth weight pups, Toxicology $300(1-2)(2012) 1-11$.

[36] R.E. Neal, J. Chen, R. Jagadapillai, H. Jang, B. Abomoelak, G. Brock, R.M. Greene, M.M. Pisano, Developmental cigarette smoke exposure: hippocampus proteome and metabolome profiles in low birth weight pups, Toxicology 317 (2014) 40-9.

[37] R. Jagadapillai, J. Chen, L. Canales, T. Birtles, M.M. Pisano, R.E. Neal, Developmental cigarette smoke exposure: kidney proteome profile alterations in low birth weight pups, Toxicology 299(2-3) (2012) 80-9. 
[38] R.E. Neal, R. Jagadapillai, J. Chen, C. Webb, K. Stocke, R.M. Greene, M.M. Pisano, Developmental cigarette smoke exposure II: Hippocampus proteome and metabolome profiles in adult offspring, Reprod Toxicol (2016).

[39] R.E. Neal, R. Jagadapillai, J. Chen, C.L. Webb, K. Stocke, C. Gambrell, R.M. Greene, M.M. Pisano, Developmental cigarette smoke exposure II: Kidney proteome profile alterations in 6 month old adult offspring, Reprod Toxicol (2016).

[40] R.E. Neal, J. Chen, C. Webb, K. Stocke, C. Gambrell, R.M. Greene, M.M. Pisano, Developmental cigarette smoke exposure II: Hepatic proteome profiles in 6 month old adult offspring, Reprod Toxicol (2016).

[41] E.R. Esposito, K.H. Horn, R.M. Greene, M.M. Pisano, An animal model of cigarette smokeinduced in utero growth retardation, Toxicology 246(2-3) (2008) 193-202.

[42] M.J. Seller, K.S. Bnait, Effects of tobacco smoke inhalation on the developing mouse embryo and fetus, Reprod Toxicol 9(5) (1995) 449-59.

[43] J.A. Bassi, P. Rosso, A.C. Moessinger, W.A. Blanc, L.S. James, Fetal growth retardation due to maternal tobacco smoke exposure in the rat, Pediatric research 18(2) (1984) 127-30.

[44] R.M. Amos-Kroohs, M.T. Williams, A.A. Braun, D.L. Graham, C.L. Webb, T.S. Birtles, R.M. Greene, C.V. Vorhees, M.M. Pisano, Neurobehavioral phenotype of C57BL/6J mice prenatally and neonatally exposed to cigarette smoke, Neurotoxicology and teratology 35 (2013) 34-45.

[45] M. Ferrini, S. Carvalho, Y.H. Cho, B. Postma, L.M. Marques, K. Pinkerton, K. Roberts, Z. Jaffar, Prenatal tobacco smoke exposure predisposes offspring mice to exacerbated allergic airway inflammation associated with altered innate effector function, Particle and fibre toxicology 14(1) (2017) 30.

[46] S.P. Ng, D.J. Conklin, A. Bhatnagar, D.D. Bolanowski, J. Lyon, J.T. Zelikoff, Prenatal exposure to cigarette smoke induces diet-and sex-dependent dyslipidemia and weight gain in adult murine offspring, Environmental health perspectives 117(7) (2009) 1042-1048.

[47] P. Drake, A.K. Driscoll, T.J. Mathews, Cigarette Smoking During Pregnancy: United States, 2016, NCHS Data Brief (305) (2018) 1-8. 
[48] S. Curtin, T. Matthews, Smoking prevalence and cessation before and during pregnancy: data from the birth certificate, 2014, National vital statistics reports: from the Centers for Disease Control and Prevention, National Center for Health Statistics, National Vital Statistics System 65(1) (2016) $1-14$.

[49] M. Osterman, J.A. Martin, T.J. Mathews, B.E. Hamilton, Expanded data from the new birth certificate, 2008, National vital statistics reports: from the Centers for Disease Control and Prevention, National Center for Health Statistics, National Vital Statistics System 59(7) (2011) 128.

[50] T. Mathews, Smoking during pregnancy in the 1990s, Natl Vital Stat Rep 49(7) (2001) 1-14.

[51] P.M. Dietz, D. Homa, L.J. England, K. Burley, V.T. Tong, S.R. Dube, J.T. Bernert, Estimates of nondisclosure of cigarette smoking among pregnant and nonpregnant women of reproductive age in the United States, American journal of epidemiology 173(3) (2010) 355-359.

[52] G. Oster, T.E. Delea, G.A. Colditz, Maternal smoking during pregnancy and expenditures on neonatal health care, Am J Prev Med 4(4) (1988) 216-9.

[53] E.K. Adams, M.F. Ayadi, C.L. Melvin, C.C. Rivera, Smoking among Medicaid insured mothers: what are the neonatal expenses?, Health care financing review 26(2) (2004) 105.

[54] E.K. Adams, C.L. Melvin, C. Raskind-Hood, P.J. Joski, E. Galactionova, Infant delivery costs related to maternal smoking: an update, Nicotine \& Tobacco Research 13(8) (2011) 627-637.

[55] H.A. Wahabi, A.A. Mandil, R.A. Alzeidan, A.A. Bahnassy, A.A. Fayed, The independent effects of second hand smoke exposure and maternal body mass index on the anthropometric measurements of the newborn, BMC Public Health 13 (2013) 1058.

[56] Z. Niu, C. Xie, X. Wen, F. Tian, P. Ding, Y. He, J. Lin, S. Yuan, X. Guo, D. Jia, W.Q. Chen, Placenta mediates the association between maternal second-hand smoke exposure during pregnancy and small for gestational age, Placenta 36(8) (2015) 876-80.

[57] M. Ramadani, B. Utomo, E.L. Achadi, H. Gunardi, Prenatal Secondhand Smoke Exposure: Correlation Between Nicotine in Umbilical Cord Blood and Neonatal Anthropometry, Osong public health and research perspectives 10(4) (2019) 234. 
[58] D. Chunxia, W. Meifang, Z. Jianhua, Z. Ruijuan, L. Xiue, Z. Zhuanzhen, Y. Linhua, Tobacco smoke exposure and the risk of childhood acute lymphoblastic leukemia and acute myeloid leukemia: A meta-analysis, Medicine 98(28) (2019).

[59] C.Y. Leung, G.M. Leung, C.M. Schooling, Early second-hand smoke exposure and child and adolescent mental health: evidence from Hong Kong's 'Children of 1997' birth cohort, Addiction $110(11)(2015)$ 1811-24.

[60] U. Food, D. Administration, Harmful and potentially harmful constituents in tobacco products and tobacco smoke; established list, Fed Regist 77(64) (2012) 20034-20037.

[61] U.D.o. Health, H. Services, Risks associated with smoking cigarettes with low machinemeasured yields of tar and nicotine, Smoking and tobacco control monograph (13) (2001).

[62] D.J. Balfour, The neurobiology of tobacco dependence: a preclinical perspective on the role of the dopamine projections to the nucleus accumbens [corrected], Nicotine Tob Res 6(6) (2004) 899912.

[63] N.I. Boyadjieva, D.K. Sarkar, The secretory response of hypothalamic beta-endorphin neurons to acute and chronic nicotine treatments and following nicotine withdrawal, Life sciences 61(6) (1997) PL59-66.

[64] H.D. Mansvelder, J.R. Keath, D.S. McGehee, Synaptic mechanisms underlie nicotine-induced excitability of brain reward areas, Neuron 33(6) (2002) 905-19.

[65] A.A. Harrison, Y.T. Liem, A. Markou, Fluoxetine combined with a serotonin-1A receptor antagonist reversed reward deficits observed during nicotine and amphetamine withdrawal in rats, Neuropsychopharmacology 25(1) (2001) 55-71.

[66] T.A. Slotkin, S.E. Lappi, E.C. McCook, B.A. Lorber, F.J. Seidler, Loss of neonatal hypoxia tolerance after prenatal nicotine exposure: implications for sudden infant death syndrome, Brain Res Bull 38(1) (1995) 69-75.

[67] S.E. Santiago, K.J. Huffman, Postnatal effects of prenatal nicotine exposure on body weight, brain size and cortical connectivity in mice, Neuroscience research 73(4) (2012) 282-291.

[68] D. Xu, L.P. Xia, L. Shen, Y.Y. Lei, L. Liu, L. Zhang, J. Magdalou, H. Wang, Prenatal nicotine exposure enhances the susceptibility to metabolic syndrome in adult offspring rats fed high-fat diet 
via alteration of HPA axis-associated neuroendocrine metabolic programming, Acta pharmacologica Sinica 34(12) (2013) 1526-34.

[69] N. Ma, C.J. Nicholson, M. Wong, A.C. Holloway, D.B. Hardy, Fetal and neonatal exposure to nicotine leads to augmented hepatic and circulating triglycerides in adult male offspring due to increased expression of fatty acid synthase, Toxicology and applied pharmacology 275(1) (2014) $1-11$.

[70] J.E. Bruin, L.D. Kellenberger, H.C. Gerstein, K.M. Morrison, A.C. Holloway, Fetal and neonatal nicotine exposure and postnatal glucose homeostasis: identifying critical windows of exposure, The Journal of endocrinology 194(1) (2007) 171-8.

[71] A. Onal, A. Uysal, S. Ulker, Y. Delen, M.E. Yurtseven, A. Evinc, Alterations of brain tissue in fetal rats exposed to nicotine in utero: possible involvement of nitric oxide and catecholamines, Neurotoxicology and teratology 26(1) (2004) 103-12.

[72] A. Sarasin, M. Schlumpf, M. Muller, I. Fleischmann, M.E. Lauber, W. Lichtensteiger, Adrenalmediated rather than direct effects of nicotine as a basis of altered sex steroid synthesis in fetal and neonatal rat, Reprod Toxicol 17(2) (2003) 153-62.

[73] P. Bonthuis, K. Cox, B. Searcy, P. Kumar, S. Tobet, E. Rissman, Of mice and rats: key species variations in the sexual differentiation of brain and behavior, Frontiers in neuroendocrinology $31(3)$ (2010) 341-358.

[74] D. Hoffmann, S.S. Hecht, R.M. Ornaf, E.L. Wynder, N'-nitrosonornicotine in tobacco, Science (New York, N.Y.) 186(4160) (1974) 265-7.

[75] W. Chamberlain, R. Severson, M. Stephenson, Levels of N-nitrosonornicotine in tobaccos grown under varying agronomic conditions, Tobacco International 186(26) (1984) 111-113.

[76] C.A. Flanagan, K.R. Koller, A.W. Wolfe, T.K. Thomas, N.L. Benowitz, C.C. Renner, C. Hughes, D.K. Hatsukami, C. Bronars, N.J. Murphy, G. Day, P.A. Decker, C.A. Patten, Fetal Exposure to Carcinogens With Tobacco Use in Pregnancy: Phase 1 MAW Study Findings, Nicotine Tob Res 18(11) (2016) 2162-2168.

[77] E. Florek, W. Piekoszewski, A. Basior, A.T. Merritt, J. Mazela, W. Lechowicz, M.K. Kornacka, L. Kramer, Effect of maternal tobacco smoking or exposure to second-hand smoke on the levels of 
4-(methylnitrosamino)-1-(3-pyridyl)-1-butanol (NNAL) in urine of mother and the first urine of newborn, J Physiol Pharmacol 62(3) (2011) 377-83.

[78] G.M. Lackmann, U. Salzberger, U. Tollner, M. Chen, S.G. Carmella, S.S. Hecht, Metabolites of a tobacco-specific carcinogen in urine from newborns, J Natl Cancer Inst 91(5) (1999) 459-65. [79] A. Milunsky, S.G. Carmella, M. Ye, S.S. Hecht, A tobacco-specific carcinogen in the fetus, Prenatal diagnosis 20(4) (2000) 307-10.

[80] S.S. Hecht, Biochemistry, biology, and carcinogenicity of tobacco-specific N-nitrosamines, Chemical research in toxicology 11(6) (1998) 559-603.

[81] P.E. Douben, PAHs: an ecotoxicological perspective, John Wiley \& Sons2003.

[82] D. Hoffmann, E.L. Wynder, The reduction of the tumorigenicity of cigarette smoke condensate by addition of sodium nitrate to tobacco, Cancer Research 27(1) (1967) 172-174.

[83] K. Torikaiu, Y. Uwano, T. Nakamori, W. Tarora, H. Takahashi, Study on tobacco components involved in the pyrolytic generation of selected smoke constituents, Food and chemical toxicology 43(4) (2005) 559-568.

[84] Y.S. Ding, X.J. Yan, R.B. Jain, E. Lopp, A. Tavakoli, G.M. Polzin, S.B. Stanfill, D.L. Ashley, C.H. Watson, Determination of 14 polycyclic aromatic hydrocarbons in mainstream smoke from US brand and non-US brand cigarettes, Environmental science \& technology 40(4) (2006) 1133-1138. [85] A.T. Vu, K.M. Taylor, M.R. Holman, Y.S. Ding, B. Hearn, C.H. Watson, Polycyclic aromatic hydrocarbons in the mainstream smoke of popular US cigarettes, Chemical research in toxicology 28(8) (2015) 1616-1626.

[86] D. Thakker, H. Yagi, W. Levin, A. Wood, A. Conney, D. Jerina, Polycyclic aromatic hydrocarbons: metabolic activation to ultimate carcinogens, Bioactivation of foreign compounds (1985) 177-242.

[87] P.H. Langlois, A.T. Hoyt, T.A. Desrosiers, P.J. Lupo, C.C. Lawson, M.A. Waters, C.M. Rocheleau, G.M. Shaw, P.A. Romitti, S.M. Gilboa, Maternal occupational exposure to polycyclic aromatic hydrocarbons and small for gestational age offspring, Occup Environ Med 71(8) (2014) 529-535. 
[88] H. Choi, L. Wang, X. Lin, J.D. Spengler, F.P. Perera, Fetal window of vulnerability to airborne polycyclic aromatic hydrocarbons on proportional intrauterine growth restriction, PloS one 7(4) (2012) e35464.

[89] T. Duarte-Salles, M.A. Mendez, H.M. Meltzer, J. Alexander, M. Haugen, Dietary benzo (a) pyrene intake during pregnancy and birth weight: associations modified by vitamin $\mathrm{C}$ intakes in the Norwegian Mother and Child Cohort Study (MoBa), Environment international 60 (2013) 217-223. [90] L. Ortiz, B. Nakamura, X. Li, B. Blumberg, U. Luderer, In utero exposure to benzo[a]pyrene increases adiposity and causes hepatic steatosis in female mice, and glutathione deficiency is protective, Toxicology letters 223(2) (2013) 260-7.

[91] K. Torikai, S. Yoshida, H. Takahashi, Effects of temperature, atmosphere and $\mathrm{pH}$ on the generation of smoke compounds during tobacco pyrolysis, Food and Chemical Toxicology 42(9) (2004) 1409-1417.

[92] M. Counts, M. Morton, S. Laffoon, R. Cox, P. Lipowicz, Smoke composition and predicting relationships for international commercial cigarettes smoked with three machine-smoking conditions, Regulatory Toxicology and Pharmacology 41(3) (2005) 185-227.

[93] J. Fowles, E. Dybing, Application of toxicological risk assessment principles to the chemical constituents of cigarette smoke, Tobacco control 12(4) (2003) 424-430.

[94] M. Chang, H. Park, M. Ha, Y.-C. Hong, Y.-H. Lim, Y. Kim, Y.J. Kim, D. Lee, E.-H. Ha, The effect of prenatal TVOC exposure on birth and infantile weight: the Mothers and Children's Environmental Health study, Pediatric research 82(3) (2017) 423.

[95] P. Franklin, M. Tan, N. Hemy, G.L. Hall, Maternal Exposure to Indoor Air Pollution and Birth Outcomes, International journal of environmental research and public health 16(8) (2019) 1364.

[96] D. Chen, S.-I. Cho, C. Chen, X. Wang, A.I. Damokosh, L. Ryan, T.J. Smith, D.C. Christiani, X. $\mathrm{Xu}$, Exposure to benzene, occupational stress, and reduced birth weight, Occupational and environmental medicine 57(10) (2000) 661-667.

[97] R.S. Schwartz, L.T. Hecking, Determination of geographic origin of agricultural products by multivariate analysis of trace element composition, Journal of Analytical Atomic Spectrometry 6(8) (1991) 637-642. 
[98] T. Xiao, J. Guha, D. Boyle, C.-Q. Liu, B. Zheng, G.C. Wilson, A. Rouleau, J. Chen, Naturally occurring thallium: a hidden geoenvironmental health hazard?, Environment International 30(4) (2004) 501-507.

[99] W.E. Stephens, A. Calder, J. Newton, Source and health implications of high toxic metal concentrations in illicit tobacco products, Environmental science \& technology 39(2) (2005) 479488.

[100] W.S. Rickert, J.C. Robinson, D.F. Bray, B. Rogers, N.E. Collishaw, Characterization of tobacco products: a comparative study of the tar, nicotine, and carbon monoxide yields of cigars, manufactured cigarettes, and cigarettes made from fine-cut tobacco, Preventive Medicine 14(2) (1985) 226-233.

[101] M. Chiba, R. Masironi, Toxic and trace elements in tobacco and tobacco smoke, Bulletin of the World Health Organization 70(2) (1992) 269.

[102] G. Schneider, V. Krivna, Multi-element analysis of tobacco and smoke condensate by instrumental neutron activation analysis and atomic absorption spectrometry, International journal of environmental analytical chemistry 53(2) (1993) 87-100.

[103] K. Kalcher, W. Kern, R. Pietsch, Cadmium and lead in the smoke of a filter cigarette, Science of the Total Environment 128(1) (1993) 21-35.

[104] J. Chmielnicka, B. Sowa, Cadmium interaction with essential metals ( $\mathrm{Zn}, \mathrm{Cu}, \mathrm{Fe})$, metabolism metallothionein, and ceruloplasmin in pregnant rats and fetuses, Ecotoxicology and environmental safety $35(3)(1996)$ 277-281.

[105] M.C. Henson, P.J. Chedrese, Endocrine disruption by cadmium, a common environmental toxicant with paradoxical effects on reproduction, Experimental biology and medicine 229(5) (2004) 383-392.

[106] A.J. Green, C. Hoyo, C.J. Mattingly, Y. Luo, J.-Y. Tzeng, S.K. Murphy, D.B. Buchwalter, A. Planchart, Cadmium exposure increases the risk of juvenile obesity: a human and zebrafish comparative study, International Journal of Obesity (2018) 1. 
[107] Y.C. Hong, S.S. Kulkarni, Y.H. Lim, E. Kim, M. Ha, H. Park, Y. Kim, B.N. Kim, N. Chang, S.Y. Oh, Y.J. Kim, C. Park, E.H. Ha, Postnatal growth following prenatal lead exposure and calcium intake, Pediatrics 134(6) (2014) 1151-9.

[108] G. Wang, J. DiBari, E. Bind, A.M. Steffens, J. Mukherjee, R.E. Azuine, G.K. Singh, X. Hong, Y. Ji, H. Ji, Association between maternal exposure to lead, maternal folate status, and intergenerational risk of childhood overweight and obesity, JAMA Network Open 2(10) (2019) e1912343-e1912343.

[109] H.A. de Boo, J.E. Harding, The developmental origins of adult disease (Barker) hypothesis, Aust N Z J Obstet Gynaecol 46(1) (2006) 4-14.

[110] D.J. Barker, C. Osmond, S.J. Simmonds, G.A. Wield, The relation of small head circumference and thinness at birth to death from cardiovascular disease in adult life, BMJ 306(6875) (1993) 422-6.

[111] G.-P. Ravelli, Z.A. Stein, M.W. Susser, Obesity in young men after famine exposure in utero and early infancy, New England Journal of Medicine 295(7) (1976) 349-353.

[112] R.C. Painter, T.J. Roseboom, O.P. Bleker, Prenatal exposure to the Dutch famine and disease in later life: an overview, Reproductive toxicology 20 (3) (2005) 345-352.

[113] Z. Yang, W. Zhao, X. Zhang, R. Mu, Y. Zhai, L. Kong, C. Chen, Impact of famine during pregnancy and infancy on health in adulthood, Obesity reviews 9 (2008) 95-99.

[114] A.C. Ravelli, J.H. Van Der Meulen, C. Osmond, D.J. Barker, O.P. Bleker, Obesity at the age of $50 \mathrm{y}$ in men and women exposed to famine prenatally, The American journal of clinical nutrition 70(5) (1999) 811-816.

[115] D.J. Barker, J.G. Eriksson, T. Forsén, C. Osmond, Fetal origins of adult disease: strength of effects and biological basis, International journal of epidemiology 31(6) (2002) 1235-1239.

[116] C. Stein, C. Fall, K. Kumaran, C. Osmond, D. Barker, V. Cox, Fetal growth and coronary heart disease in south India, The Lancet 348(9037) (1996) 1269-1273.

[117] T. Roseboom, S. de Rooij, R. Painter, The Dutch famine and its long-term consequences for adult health, Early human development 82(8) (2006) 485-491. 
[118] A.F. van Abeelen, M.V. Veenendaal, R.C. Painter, S.R. de Rooij, M.G. Dijkgraaf, P.M. Bossuyt, S.G. Elias, D.E. Grobbee, C.S. Uiterwaal, T.J. Roseboom, Survival effects of prenatal famine exposure, The American journal of clinical nutrition 95(1) (2011) 179-183.

[119] J.W. Rich-Edwards, G.A. Colditz, M.J. Stampfer, W.C. Willett, M.W. Gillman, C.H. Hennekens, F.E. Speizer, J.E. Manson, Birthweight and the risk for type 2 diabetes mellitus in adult women, Annals of internal medicine 130(4_Part_1) (1999) 278-284.

[120] S.K. Bhargava, H.S. Sachdev, C.H. Fall, C. Osmond, R. Lakshmy, D.J. Barker, S.K.D. Biswas, S. Ramji, D. Prabhakaran, K.S. Reddy, Relation of serial changes in childhood body-mass index to impaired glucose tolerance in young adulthood, New England Journal of Medicine 350(9) (2004) 865-875.

[121] P. Whincup, D. Cook, F. Adshead, S. Taylor, M. Walker, O. Papacosta, K. Alberti, Childhood size is more strongly related than size at birth to glucose and insulin levels in 10-11-year-old children, Diabetologia 40(3) (1997) 319-326.

[122] C. Li, M.S. Johnson, M.I. Goran, Effects of low birth weight on insulin resistance syndrome in caucasian and African-American children, Diabetes Care 24(12) (2001) 2035-42.

[123] J. Eriksson, T. Forsen, J. Tuomilehto, C. Osmond, D. Barker, Fetal and childhood growth and hypertension in adult life, Hypertension 36(5) (2000) 790-794.

[124] R.M. Martin, A. McCarthy, G.D. Smith, D.P. Davies, Y. Ben-Shlomo, Infant nutrition and blood pressure in early adulthood: the Barry Caerphilly Growth study, The American journal of clinical nutrition 77(6) (2003) 1489-97.

[125] C.M. Law, A.W. Shiell, C.A. Newsome, H.E. Syddall, E.A. Shinebourne, P.M. Fayers, C.N. Martyn, M. de Swiet, Fetal, infant, and childhood growth and adult blood pressure: a longitudinal study from birth to 22 years of age, Circulation 105(9) (2002) 1088-92.

[126] M. Desai, N.J. Crowther, A. Lucas, C.N. Hales, Organ-selective growth in the offspring of protein-restricted mothers, Br J Nutr 76(4) (1996) 591-603.

[127] S.M. Woodall, B.M. Johnston, B.H. Breier, P.D. Gluckman, Chronic maternal undernutrition in the rat leads to delayed postnatal growth and elevated blood pressure of offspring, Pediatric research 40(3) (1996) 438-43. 
[128] S.E. Ozanne, C.N. Hales, The long-term consequences of intra-uterine protein malnutrition for glucose metabolism, Proc Nutr Soc 58(3) (1999) 615-9.

[129] S.C. Langley-Evans, S.J. Welham, R.C. Sherman, A.A. Jackson, Weanling rats exposed to maternal low-protein diets during discrete periods of gestation exhibit differing severity of hypertension, Clin Sci (Lond) 91(5) (1996) 607-15.

[130] A.R. Pinheiro, I.D. Salvucci, M.B. Aguila, C.A. Mandarim-de-Lacerda, Protein restriction during gestation and/or lactation causes adverse transgenerational effects on biometry and glucose metabolism in F1 and F2 progenies of rats, Clinical science 114(5) (2008) 381-392.

[131] L.M. Berends, L. Dearden, Y.C.L. Tung, P. Voshol, D.S. Fernandez-Twinn, S.E. Ozanne, Programming of central and peripheral insulin resistance by low birthweight and postnatal catchup growth in male mice, Diabetologia 61(10) (2018) 2225-2234.

[132] L. Berends, D. Fernandez-Twinn, M. Martin-Gronert, R. Cripps, S. Ozanne, Catch-up growth following intra-uterine growth-restriction programmes an insulin-resistant phenotype in adipose tissue, International journal of obesity 37(8) (2013) 1051.

[133] E. Zambrano, P. Martinez-Samayoa, C. Bautista, M. Deas, L. Guillen, G. RodriguezGonzalez, C. Guzman, F. Larrea, P. Nathanielsz, Sex differences in transgenerational alterations of growth and metabolism in progeny (F2) of female offspring (F1) of rats fed a low protein diet during pregnancy and lactation, The Journal of physiology 566(1) (2005) 225-236.

[134] D.C. Benyshek, C.S. Johnston, J.F. Martin, Post-natal diet determines insulin resistance in fetally malnourished, low birthweight rats (F1) but diet does not modify the insulin resistance of their offspring (F2), Life sciences 74(24) (2004) 3033-3041.

[135] K. Godfrey, S. Robinson, D.J. Barker, C. Osmond, V. Cox, Maternal nutrition in early and late pregnancy in relation to placental and fetal growth, BMJ 312(7028) (1996) 410-4.

[136] V.M. Moore, M.J. Davies, K.J. Willson, A. Worsley, J.S. Robinson, Dietary composition of pregnant women is related to size of the baby at birth, The Journal of nutrition $134(7)(2004) 1820$ 6.

[137] S. Rao, C.S. Yajnik, A. Kanade, C.H. Fall, B.M. Margetts, A.A. Jackson, R. Shier, S. Joshi, S. Rege, H. Lubree, B. Desai, Intake of micronutrient-rich foods in rural Indian mothers is 
associated with the size of their babies at birth: Pune Maternal Nutrition Study, The Journal of nutrition 131(4) (2001) 1217-24.

[138] E. Oken, E.B. Levitan, M.W. Gillman, Maternal smoking during pregnancy and child overweight: systematic review and meta-analysis, Int J Obes (Lond) 32(2) (2008) 201-10.

[139] E. Raum, J. Kupper-Nybelen, A. Lamerz, J. Hebebrand, B. Herpertz-Dahlmann, H. Brenner, Tobacco smoke exposure before, during, and after pregnancy and risk of overweight at age 6, Obesity (Silver Spring) 19(12) (2011) 2411-7.

[140] E. Oken, S.Y. Huh, E.M. Taveras, J.W. Rich-Edwards, M.W. Gillman, Associations of maternal prenatal smoking with child adiposity and blood pressure, Obes Res 13(11) (2005) 2021 8.

[141] A.M. Toschke, S.M. Montgomery, U. Pfeiffer, R. von Kries, Early intrauterine exposure to tobacco-inhaled products and obesity, American journal of epidemiology 158(11) (2003) 1068-74. [142] R. von Kries, A.M. Toschke, B. Koletzko, W. Slikker, Jr., Maternal smoking during pregnancy and childhood obesity, American journal of epidemiology 156(10) (2002) 954-61.

[143] M. Weitzman, S. Cook, P. Auinger, T.A. Florin, S. Daniels, M. Nguyen, J.P. Winickoff, Tobacco smoke exposure is associated with the metabolic syndrome in adolescents, Circulation 112(6) (2005) 862-9.

[144] R. McConnell, E. Shen, F.D. Gilliland, M. Jerrett, J. Wolch, C.-C. Chang, F. Lurmann, K. Berhane, A longitudinal cohort study of body mass index and childhood exposure to secondhand tobacco smoke and air pollution: the Southern California Children's Health Study, Environmental health perspectives 123(4) (2014) 360-366.

[145] E. Raum, J. Küpper-Nybelen, A. Lamerz, J. Hebebrand, B. Herpertz-Dahlmann, H. Brenner, Tobacco smoke exposure before, during, and after pregnancy and risk of overweight at age 6, Obesity 19(12) (2011) 2411-2417.

[146] H.-J. Chen, G.-L. Li, A. Sun, D.-S. Peng, W.-X. Zhang, Y.-E. Yan, Age Differences in the Relationship between Secondhand Smoke Exposure and Risk of Metabolic Syndrome: A MetaAnalysis, International journal of environmental research and public health 16(8) (2019) 1409. 
[147] C.L. Davis, M.S. Tingen, J. Jia, F. Sherman, C.F. Williams, K. Bhavsar, N. Wood, J. Kobleur, J.L. Waller, Passive smoke exposure and its effects on cognition, sleep, and health outcomes in overweight and obese children, Childhood Obesity 12(2) (2016) 119-125.

[148] L. Albers, C. Sobotzki, O. Kuß, T. Ajslev, R.F. Batista, H. Bettiol, B. Brabin, S.L. Buka, V.C. Cardoso, V.L. Clifton, Maternal smoking during pregnancy and offspring overweight: is there a dose-response relationship? An individual patient data meta-analysis, International Journal of Obesity 42(7) (2018) 1249.

[149] C.N. Hales, D.J. Barker, Type 2 (non-insulin-dependent) diabetes mellitus: the thrifty phenotype hypothesis. 1992, Int J Epidemiol 42(5) (2013) 1215-22.

[150] J.C. Wells, The thrifty phenotype as an adaptive maternal effect, Biol Rev Camb Philos Soc 82(1) (2007) 143-72.

[151] P.D. Gluckman, M.A. Hanson, Developmental origins of disease paradigm: a mechanistic and evolutionary perspective, Pediatric research 56(3) (2004) 311-7.

[152] D.I. Phillips, Insulin resistance as a programmed response to fetal undernutrition, Diabetologia 39(9) (1996) 1119-22.

[153] P.L. Huang, A comprehensive definition for metabolic syndrome, Dis Model Mech 2(5-6) (2009) 231-7.

[154] K.G. Alberti, P.Z. Zimmet, Definition, diagnosis and classification of diabetes mellitus and its complications. Part 1: diagnosis and classification of diabetes mellitus provisional report of a WHO consultation, Diabet Med 15(7) (1998) 539-53.

[155] S.M. Grundy, J.I. Cleeman, S.R. Daniels, K.A. Donato, R.H. Eckel, B.A. Franklin, D.J. Gordon, R.M. Krauss, P.J. Savage, S.C. Smith, Jr., J.A. Spertus, F. Costa, A. American Heart, L. National Heart, I. Blood, Diagnosis and management of the metabolic syndrome: an American Heart Association/National Heart, Lung, and Blood Institute Scientific Statement, Circulation 112(17) (2005) 2735-52.

[156] P. Zimmet, D. Magliano, Y. Matsuzawa, G. Alberti, J. Shaw, The metabolic syndrome: a global public health problem and a new definition, J Atheroscler Thromb 12(6) (2005) 295-300. 
[157] J.X. Moore, N. Chaudhary, T. Akinyemiju, Metabolic Syndrome Prevalence by Race/Ethnicity and Sex in the United States, National Health and Nutrition Examination Survey, 1988-2012, Prev Chronic Dis 14 (2017) E24.

[158] C.M. Hales, M.D. Carroll, C.D. Fryar, C.L. Ogden, Prevalence of Obesity Among Adults and Youth: United States, 2015-2016, NCHS Data Brief (288) (2017) 1-8.

[159] M. Wideroe, T. Vik, G. Jacobsen, L.S. Bakketeig, Does maternal smoking during pregnancy cause childhood overweight?, Paediatr Perinat Epidemiol 17(2) (2003) 171-9.

[160] T. Ino, Maternal smoking during pregnancy and offspring obesity: meta-analysis, Pediatr Int $52(1)(2010) 94-9$.

[161] L.A. Cupul-Uicab, R. Skjaerven, K. Haug, G.S. Travlos, R.E. Wilson, M. Eggesbo, J.A. Hoppin, K.W. Whitworth, M.P. Longnecker, Exposure to tobacco smoke in utero and subsequent plasma lipids, ApoB, and CRP among adult women in the MoBa cohort, Environmental health perspectives 120(11) (2012) 1532-7.

[162] A. Al Mamun, D.A. Lawlor, R. Alati, M.J. O'Callaghan, G.M. Williams, J.M. Najman, Does maternal smoking during pregnancy have a direct effect on future offspring obesity? Evidence from a prospective birth cohort study, American journal of epidemiology 164(4) (2006) 317-25.

[163] G. Koshy, A. Delpisheh, B.J. Brabin, Dose response association of pregnancy cigarette smoke exposure, childhood stature, overweight and obesity, Eur J Public Health 21(3) (2011) 28691.

[164] S.D. Leary, G.D. Smith, I.S. Rogers, J.J. Reilly, J.C. Wells, A.R. Ness, Smoking during pregnancy and offspring fat and lean mass in childhood, Obesity (Silver Spring) 14(12) (2006) 2284-93.

[165] L. Hogberg, S. Cnattingius, C. Lundholm, B.M. D'Onofrio, N. Langstrom, A.N. Iliadou, Effects of maternal smoking during pregnancy on offspring blood pressure in late adolescence, $\mathrm{J}$ Hypertens 30(4) (2012) 693-9.

[166] C.C. Geerts, D.E. Grobbee, C.K. van der Ent, B.M. de Jong, M.M. van der Zalm, N. van PutteKatier, J.L. Kimpen, C.S. Uiterwaal, Tobacco smoke exposure of pregnant mothers and blood 
pressure in their newborns: results from the wheezing illnesses study Leidsche Rijn birth cohort, Hypertension 50(3) (2007) 572-8.

[167] E. Thiering, I. Bruske, J. Kratzsch, J. Thiery, S. Sausenthaler, C. Meisinger, S. Koletzko, C.P. Bauer, B. Schaaf, A. von Berg, D. Berdel, I. Lehmann, O. Herbarth, U. Kramer, H.E. Wichmann, J. Heinrich, Giniplus, L.I.S. Groups, Prenatal and postnatal tobacco smoke exposure and development of insulin resistance in 10 year old children, Int J Hyg Environ Health 214(5) (2011) 361-8.

[168] M. Gordillo, T. Evans, V. Gouon-Evans, Orchestrating liver development, Development 142(12) (2015) 2094-2108.

[169] P. Schmid, W.A. Schulz, Coexpression of the C-MYC protooncogene with a-fetoprotein and albumin in fetal mouse liver, Differentiation; research in biological diversity 45(2) (1990) 96-102. [170] S. Nava, M. Westgren, M. Jaksch, A. Tibell, U. Broomé, B.-G. Ericzon, S. SumitranHolgersson, Characterization of cells in the developing human liver, Differentiation; research in biological diversity 73(5) (2005) 249-260.

[171] N. Shiojiri, Enzymo-and immunocytochemical analyses of the differentiation of liver cells in the prenatal mouse, Development 62(1) (1981) 139-152.

[172] S. Cascio, K.S. Zaret, Hepatocyte differentiation initiates during endodermal-mesenchymal interactions prior to liver formation, Development 113(1) (1991) 217-225.

[173] A.M. Müller, A. Medvinsky, J. Strouboulis, F. Grosveld, E. Dzierzakt, Development of hematopoietic stem cell activity in the mouse embryo, Immunity 1(4) (1994) 291-301.

[174] G. Migliaccio, A. Migliaccio, S. Petti, F. Mavilio, G. Russo, D. Lazzaro, U. Testa, M. Marinucci, C. Peschle, Human embryonic hemopoiesis. Kinetics of progenitors and precursors underlying the yolk sac----liver transition, The Journal of clinical investigation 78(1) (1986) 51-60.

[175] B.H. Ruebner, T.A. Blankenberg, D.A. Burrows, W. Soohoo, J.K. Lund, Development and transformation of the ductal plate in the developing human liver, Pediatric pathology 10(1-2) (1990) $55-68$.

[176] Y. Haruna, K. Saito, S. Spaulding, M.A. Nalesnik, M.A. Gerber, Identification of bipotential progenitor cells in human liver development, Hepatology 23(3) (1996) 476-481. 
[177] T. Terada, Y. Nakanuma, Development of human intrahepatic peribiliary glands. Histological, keratin immunohistochemical, and mucus histochemical analyses, Laboratory investigation; a journal of technical methods and pathology 68(3) (1993) 261-269.

[178] J.E. Moscovitz, L.M. Aleksunes, Establishment of metabolism and transport pathways in the rodent and human fetal liver, International journal of molecular sciences 14(12) (2013) 2380123827.

[179] T. Kietzmann, Metabolic zonation of the liver: The oxygen gradient revisited, Redox biology $11(2017) 622-630$.

[180] P.B. Iynedjian, S. Marie, A. Gjinovci, B. Genin, S.-P. Deng, L. Buhler, P. Morel, G. Mentha, Glucokinase and cytosolic phosphoenolpyruvate carboxykinase (GTP) in the human liver. Regulation of gene expression in cultured hepatocytes, The Journal of clinical investigation 95(5) (1995) 1966-1973.

[181] C.B. Quick, R.A. Fisher, H. Harris, A kinetic study of the isozymes determined by the three human phosphoglucomutase loci PGM1, PGM2 and PGM3, European journal of biochemistry 42(2) (1974) 511-517.

[182] Q. Yu, X. Zheng, The crystal structure of human UDP-glucose pyrophosphorylase reveals a latch effect that influences enzymatic activity, Biochemical Journal 442(2) (2012) 283-291.

[183] M.M. Adeva-Andany, N. Pérez-Felpete, C. Fernández-Fernández, C. Donapetry-García, C. Pazos-García, Liver glucose metabolism in humans, Bioscience reports (2016) BSR20160385.

[184] D. Cifuentes, C. Martínez-Pons, M. García-Rocha, A. Galina, L.R. de Pouplana, J.J. Guinovart, Hepatic Glycogen Synthesis in the Absence of Glucokinase THE CASE OF EMBRYONIC LIVER, Journal of Biological Chemistry 283(9) (2008) 5642-5649.

[185] R.M. McDevitt, S.J. Bott, M. Harding, W.A. Coward, L.J. Bluck, A.M. Prentice, De novo lipogenesis during controlled overfeeding with sucrose or glucose in lean and obese women, The American journal of clinical nutrition 74(6) (2001) 737-746.

[186] S. Egger, A. Chaikuad, K.L. Kavanagh, U. Oppermann, B. Nidetzky, UDP-glucose dehydrogenase: structure and function of a potential drug target, Portland Press Ltd., 2010. 
[187] A. Stincone, A. Prigione, T. Cramer, M.M. Wamelink, K. Campbell, E. Cheung, V. OlinSandoval, N.M. Grüning, A. Krüger, M. Tauqeer Alam, The return of metabolism: biochemistry and physiology of the pentose phosphate pathway, Biological Reviews 90(3) (2015) 927-963.

[188] L.P. Bechmann, R.A. Hannivoort, G. Gerken, G.S. Hotamisligil, M. Trauner, A. Canbay, The interaction of hepatic lipid and glucose metabolism in liver diseases, Journal of hepatology 56(4) (2012) 952-964.

[189] J.C. Hutton, R.M. O'Brien, Glucose-6-phosphatase catalytic subunit gene family, Journal of Biological Chemistry 284(43) (2009) 29241-29245.

[190] J.C. Wallace, S. Jitrapakdee, A. Chapman-Smith, Pyruvate carboxylase, The international journal of biochemistry \& cell biology 30(1) (1998) 1-5.

[191] P. She, M. Shiota, K.D. Shelton, R. Chalkley, C. Postic, M.A. Magnuson, Phosphoenolpyruvate carboxykinase is necessary for the integration of hepatic energy metabolism, Molecular and cellular biology 20(17) (2000) 6508-6517.

[192] F. Marcus, J. Rittenhouse, B. Gontero, P.B. Harrsch, Function, structure and evolution of fructose-1, 6-bisphosphatase, Archivos de biologia y medicina experimentales 20(3-4) (1987) 371378.

[193] V. Chandramouli, K. Ekberg, W.C. Schumann, S.C. Kalhan, J. Wahren, B.R. Landau, Quantifying gluconeogenesis during fasting, American Journal of Physiology-Endocrinology And Metabolism 273(6) (1997) E1209-E1215.

[194] L. Rui, Energy metabolism in the liver, Comprehensive physiology 4(1) (2011) 177-197.

[195] L. Abu-Elheiga, M.M. Matzuk, P. Kordari, W. Oh, T. Shaikenov, Z. Gu, S.J. Wakil, Mutant mice lacking acetyl-CoA carboxylase 1 are embryonically lethal, Proceedings of the National Academy of Sciences 102(34) (2005) 12011-12016.

[196] S.S. Chirala, S.J. Wakil, Structure and function of animal fatty acid synthase, Lipids 39(11) (2004) 1045-1053.

[197] R.M. McDevitt, S.J. Bott, M. Harding, W.A. Coward, L.J. Bluck, A.M. Prentice, De novo lipogenesis during controlled overfeeding with sucrose or glucose in lean and obese women-, The American journal of clinical nutrition 74(6) (2001) 737-746. 
[198] S.M. Houten, R.J. Wanders, A general introduction to the biochemistry of mitochondrial fatty acid $\beta$-oxidation, Journal of inherited metabolic disease 33(5) (2010) 469-477.

[199] M.J. Coon, A. Vaz, L.L. Bestervelt, Cytochrome P450 2: peroxidative reactions of diversozymes, The FASEB Journal 10(4) (1996) 428-434.

[200] P. Danielson, The cytochrome P450 superfamily: biochemistry, evolution and drug metabolism in humans, Current drug metabolism 3(6) (2002) 561-597.

[201] D.R. Nelson, The cytochrome p450 homepage, Human genomics 4(1) (2009) 59.

[202] H. Sezutsu, G. Le Goff, R. Feyereisen, Origins of P450 diversity, Philosophical Transactions of the Royal Society B: Biological Sciences 368(1612) (2013) 20120428.

[203] J.G. Scott, N. Liu, Z. Wen, Insect cytochromes P450: diversity, insecticide resistance and tolerance to plant toxins, Comparative Biochemistry and Physiology Part C: Pharmacology, Toxicology and Endocrinology 121(1-3) (1998) 147-155.

[204] C. Xu, C.Y.-T. Li, A.-N.T. Kong, Induction of phase I, II and III drug metabolism/transport by xenobiotics, Archives of pharmacal research 28(3) (2005) 249.

[205] P.G. Wells, P.I. Mackenzie, J.R. Chowdhury, C. Guillemette, P.A. Gregory, Y. Ishii, A.J. Hansen, F.K. Kessler, P.M. Kim, N.R. Chowdhury, Glucuronidation and the UDPglucuronosyltransferases in health and disease, Drug metabolism and disposition 32(3) (2004) 281-290.

[206] R.H. Tukey, C.P. Strassburg, Human UDP-glucuronosyltransferases: metabolism, expression, and disease, Annual review of pharmacology and toxicology 40(1) (2000) 581-616.

[207] L.-Q. Wang, M.O. James, Inhibition of sulfotransferases by xenobiotics, Current drug metabolism 7(1) (2006) 83-104.

[208] E. Banoglu, Current status of the cytosolic sulfotransferases in the metabolic activation of promutagens and procarcinogens, Current drug metabolism 1(1) (2000) 1-30.

[209] J.D. Hayes, D.J. Pulford, The glut athione S-transferase supergene family: regulation of GST and the contribution of the Isoenzymes to cancer chemoprotection and drug resistance part I, Critical reviews in biochemistry and molecular biology 30(6) (1995) 445-520. 
[210] L. Lavoie, A. Tremblay, M. Mirault, Distinct oxidoresistance phenotype of human T47D cells transfected by rat glutathione S-transferase Yc expression vectors, Journal of Biological Chemistry 267(6) (1992) 3632-3636.

[211] K. Berhane, M. Widersten, Å. Engström, J.W. Kozarich, B. Mannervik, Detoxication of base propenals and other alpha, beta-unsaturated aldehyde products of radical reactions and lipid peroxidation by human glutathione transferases, Proceedings of the National Academy of Sciences $91(4)(1994)$ 1480-1484.

[212] P. Jancova, P. Anzenbacher, E. Anzenbacherova, Phase II drug metabolizing enzymes, Biomed Pap Med Fac Univ Palacky Olomouc Czech Repub 154(2) (2010) 103-116.

[213] J.A. Agundez, Polymorphisms of human N-acetyltransferases and cancer risk, Current drug metabolism 9(6) (2008) 520-531.

[214] E.J. Lammer, G.M. Shaw, D.M. lovannisci, R.H. Finnell, Periconceptional multivitamin intake during early pregnancy, genetic variation of acetyl-N-transferase 1 (NAT1), and risk for orofacial clefts, Birth Defects Research Part A: Clinical and Molecular Teratology 70(11) (2004) 846-852. [215] J. Axelrod, R. Tomchick, Enzymatic O-methylation of epinephrine and other catechols, The Journal of biological chemistry 233(3) (1958) 702-705.

[216] J.D. Yager, Mechanisms of estrogen carcinogenesis: The role of E2/E1-quinone metabolites suggests new approaches to preventive intervention-A review, Steroids 99 (2015) 56-60.

[217] R. Weinshilboum, Thiopurine pharmacogenetics: clinical and molecular studies of thiopurine methyltransferase, Drug Metabolism and Disposition 29(4) (2001) 601-605.

[218] R.M. Weinshilboum, D.M. Otterness, C.L. Szumlanski, Methylation pharmacogenetics: catechol O-methyltransferase, thiopurine methyltransferase, and histamine $\mathrm{N}$-methyltransferase, Annual review of pharmacology and toxicology 39(1) (1999) 19-52.

[219] R. Hines, Developmental expression of drug metabolizing enzymes: impact on disposition in neonates and young children, International journal of pharmaceutics 452(1-2) (2013) 3-7.

[220] D. Lacroix, M. Sonnier, A. Moncion, G. Cheron, T. Cresteil, Expression of CYP3A in the human liver-evidence that the shift between CYP3A7 and CYP3A4 occurs immediately after birth, European Journal of Biochemistry 247(2) (1997) 625-634. 
[221] J.C. Stevens, R.N. Hines, C. Gu, S.B. Koukouritaki, J.R. Manro, P.J. Tandler, M.J. Zaya, Developmental expression of the major human hepatic CYP3A enzymes, Journal of Pharmacology and Experimental Therapeutics 307(2) (2003) 573-582.

[222] S. Dubaisi, J.A. Caruso, R. Gaedigk, C.A. Vyhlidal, P.C. Smith, R.N. Hines, T.A. Kocarek, M. Runge-Morris, Developmental Expression of the Cytosolic Sulfotransferases in Human Liver, Drug Metabolism and Disposition 47(6) (2019) 592-600.

[223] Z. Duanmu, A. Weckle, S.B. Koukouritaki, R.N. Hines, J.L. Falany, C.N. Falany, T.A. Kocarek, M. Runge-Morris, Developmental expression of aryl, estrogen, and hydroxysteroid sulfotransferases in pre-and postnatal human liver, Journal of Pharmacology and Experimental Therapeutics 316(3) (2006) 1310-1317.

[224] E.L. Croom, J.C. Stevens, R.N. Hines, A.D. Wallace, E. Hodgson, Human hepatic CYP2B6 developmental expression: the impact of age and genotype, Biochemical pharmacology $78(2)$ (2009) 184-190.

[225] E.V. Barker, R. Hume, A. Hallas, W. Coughtrie, Dehydroepiandrosterone sulfotransferase in the developing human fetus: quantitative biochemical and immunological characterization of the hepatic, renal, and adrenal enzymes, Endocrinology 134(2) (1994) 982-989.

[226] D.F. Jelinek, S. Andersson, C.A. Slaughter, D.W. Russell, Cloning and regulation of cholesterol 7 alpha-hydroxylase, the rate-limiting enzyme in bile acid biosynthesis, Journal of Biological Chemistry 265(14) (1990) 8190-8197.

[227] D.W. Russell, The enzymes, regulation, and genetics of bile acid synthesis, Annual review of biochemistry 72 (2003) 137-74.

[228] S.J. Mihalik, S.J. Steinberg, Z. Pei, J. Park, D.G. Kim, A.K. Heinzer, G. Dacremont, R.J. Wanders, D.A. Cuebas, K.D. Smith, Participation of two members of the very long-chain acyl-CoA synthetase family in bile acid synthesis and recycling, Journal of Biological Chemistry 277(27) (2002) 24771-24779.

[229] C.N. Falany, M.R. Johnson, S. Barnes, R.B. Diasio, Glycine and taurine conjugation of bile acids by a single enzyme. Molecular cloning and expression of human liver bile acid CoA: amino acid N-acyltransferase, Journal of Biological Chemistry 269(30) (1994) 19375-19379. 
[230] A. Radominska, K. Comer, P. Zimniak, J. Falany, M. Iscan, C. Falany, Human liver steroid sulphotransferase sulphates bile acids, Biochemical Journal 272(3) (1990) 597.

[231] T. Pillot, M. Ouzzine, S. Fournel-Gigleux, C. Lafaurie, A. Radominska, B. Burchell, G. Siest, J. Magdalou, Glucuronidation of hyodeoxycholic acid in human liver. Evidence for a selective role of UDP-glucuronosyltransferase 2B4, Journal of Biological Chemistry 268(34) (1993) 2563625642.

[232] J.M. Ridlon, D.J. Kang, P.B. Hylemon, J.S. Bajaj, Bile acids and the gut microbiome, Current opinion in gastroenterology 30(3) (2014) 332-8.

[233] G. Xie, W. Zhong, H. Li, Q. Li, Y. Qiu, X. Zheng, H. Chen, X. Zhao, S. Zhang, Z. Zhou, S.H. Zeisel, W. Jia, Alteration of bile acid metabolism in the rat induced by chronic ethanol consumption, FASEB journal : official publication of the Federation of American Societies for Experimental Biology 27(9) (2013) 3583-93.

[234] C. Jiang, C. Xie, F. Li, L. Zhang, R.G. Nichols, K.W. Krausz, J. Cai, Y. Qi, Z.Z. Fang, S. Takahashi, N. Tanaka, D. Desai, S.G. Amin, I. Albert, A.D. Patterson, F.J. Gonzalez, Intestinal farnesoid $\mathrm{X}$ receptor signaling promotes nonalcoholic fatty liver disease, The Journal of clinical investigation 125(1) (2015) 386-402.

[235] F. Li, C. Jiang, K.W. Krausz, Y. Li, I. Albert, H. Hao, K.M. Fabre, J.B. Mitchell, A.D. Patterson, F.J. Gonzalez, Microbiome remodelling leads to inhibition of intestinal farnesoid X receptor signalling and decreased obesity, Nat Commun 4 (2013) 2384.

[236] S.I. Sayin, A. Wahlstrom, J. Felin, S. Jantti, H.U. Marschall, K. Bamberg, B. Angelin, T. Hyotylainen, M. Oresic, F. Backhed, Gut microbiota regulates bile acid metabolism by reducing the levels of tauro-beta-muricholic acid, a naturally occurring FXR antagonist, Cell Metab 17(2) (2013) 225-35.

[237] J. Nicholas, D. Barron, The use of sodium amytal in the production of anesthesia in the rat, Journal of Pharmacology and Experimental Therapeutics 46(1) (1932) 125-129.

[238] H.G. HOLCK, M.A. KANÂN, L.M. Mills, E.L. Smith, Studies upon the sex-difference in rats in tolerance to certain barbiturates and to nicotine, Journal of Pharmacology and Experimental Therapeutics 60(3) (1937) 323-346. 
[239] K. Jarukamjorn, T. Sakuma, A. Jaruchotikamol, Y. Ishino, M. Oguro, N. Nemoto, Modified expression of cytochrome P450 mRNAs by growth hormone in mouse liver, Toxicology 219(1-3) (2006) 97-105.

[240] M.C. Ramirez, L. Zubeldía-Brenner, V. Wargon, A.M. Ornstein, D. Becu-Villalobos, Expression and methylation status of female-predominant GH-dependent liver genes are modified by neonatal androgenization in female mice, Molecular and cellular endocrinology 382(2) (2014) 825-834.

[241] P. Hao, D.J. Waxman, Functional Roles of Sex-Biased, Growth Hormone-Regulated MicroRNAs miR-1948 and miR-802 in Young Adult Mouse Liver, Endocrinology 159(3) (2018) 1377-1392.

[242] M.G. Holloway, Y. Cui, E.V. Laz, A. Hosui, L. Hennighausen, D.J. Waxman, Loss of sexually dimorphic liver gene expression upon hepatocyte-specific deletion of Stat5a-Stat5b locus, Endocrinology 148(5) (2007) 1977-1986.

[243] M.G. Holloway, G.D. Miles, A.A. Dombkowski, D.J. Waxman, Liver-specific hepatocyte nuclear factor-4 $\alpha$ deficiency: greater impact on gene expression in male than in female mouse liver, Molecular Endocrinology 22(5) (2008) 1274-1286.

[244] M.G. Holloway, E.V. Laz, D.J. Waxman, Codependence of growth hormone-responsive, sexually dimorphic hepatic gene expression on signal transducer and activator of transcription $5 b$ and hepatic nuclear factor 4a, Molecular Endocrinology 20(3) (2006) 647-660.

[245] N. Chalasani, Z. Younossi, J.E. Lavine, A.M. Diehl, E.M. Brunt, K. Cusi, M. Charlton, A.J. Sanyal, The diagnosis and management of non-alcoholic fatty liver disease: Practice Guideline by the American Association for the Study of Liver Diseases, American College of Gastroenterology, and the American Gastroenterological Association, Hepatology 55(6) (2012) 2005-2023.

[246] V. Nobili, A. Alisi, K.P. Newton, J.B. Schwimmer, Comparison of the phenotype and approach to pediatric vs adult patients with nonalcoholic fatty liver disease, Gastroenterology $150(8)$ (2016) 1798-1810. 
[247] Z.M. Younossi, A.B. Koenig, D. Abdelatif, Y. Fazel, L. Henry, M. Wymer, Global epidemiology of nonalcoholic fatty liver disease-meta-analytic assessment of prevalence, incidence, and outcomes, Hepatology 64(1) (2016) 73-84.

[248] R.J. Wong, M. Aguilar, R. Cheung, R.B. Perumpail, S.A. Harrison, Z.M. Younossi, A. Ahmed, Nonalcoholic steatohepatitis is the second leading etiology of liver disease among adults awaiting liver transplantation in the United States, Gastroenterology 148(3) (2015) 547-555.

[249] A. Hamabe, H. Uto, Y. Imamura, K. Kusano, S. Mawatari, K. Kumagai, T. Kure, T. Tamai, A. Moriuchi, T. Sakiyama, Impact of cigarette smoking on onset of nonalcoholic fatty liver disease over a 10-year period, Journal of gastroenterology 46(6) (2011) 769-778.

[250] C.O. Zein, A. Unalp, R. Colvin, Y.-C. Liu, A.J. McCullough, N.S.C.R. Network, Smoking and severity of hepatic fibrosis in nonalcoholic fatty liver disease, Journal of hepatology 54(4) (2011) 753-759.

[251] C.-X. Zhang, L.-K. Guo, Y.-M. Qin, G.-Y. Li, Association of polymorphisms of adiponectin gene promoter-11377C/G, glutathione peroxidase-1 gene C594T, and cigarette smoking in nonalcoholic fatty liver disease, Journal of the Chinese Medical Association 79(4) (2016) 195-204. [252] E.L. Anderson, L.D. Howe, H.E. Jones, J.P. Higgins, D.A. Lawlor, A. Fraser, The prevalence of non-alcoholic fatty liver disease in children and adolescents: a systematic review and metaanalysis, PloS one 10(10) (2015) e0140908.

[253] C. Talia, P. Filis, U. Soffientini, B. Lucendo-Villarin, A. Douglas, D. Hay, S. Shaw, J. Iredale, M. Swortwood, M. Huestis, In utero exposure to cigarette smoke and NAFLD pathways: Sex and age specific effects in the human fetus, Toxicology letters, ELSEVIER IRELAND LTD ELSEVIER HOUSE, BROOKVALE PLAZA, EAST PARK SHANNON, CO ..., 2018, pp. S63-S64.

[254] A. Alisi, N. Panera, C. Agostoni, V. Nobili, Intrauterine growth retardation and nonalcoholic fatty liver disease in children, International journal of endocrinology 2011 (2011).

[255] X. Li, SIRT1 and energy metabolism, Acta Biochim Biophys Sin 45(1) (2013) 51-60.

[256] J.K. Kemper, Z. Xiao, B. Ponugoti, J. Miao, S. Fang, D. Kanamaluru, S. Tsang, S.Y. Wu, C.M. Chiang, T.D. Veenstra, FXR acetylation is normally dynamically regulated by $\mathrm{p} 300$ and SIRT1 but constitutively elevated in metabolic disease states, Cell Metab 10(5) (2009) 392-404. 
[257] A. Kauppinen, T. Suuronen, J. Ojala, K. Kaarniranta, A. Salminen, Antagonistic crosstalk between NF-kappaB and SIRT1 in the regulation of inflammation and metabolic disorders, Cell Signal 25(10) (2013) 1939-48.

[258] Y. Liu, R. Dentin, D. Chen, S. Hedrick, K. Ravnskjaer, S. Schenk, J. Milne, D.J. Meyers, P. Cole, J. Yates, 3rd, J. Olefsky, L. Guarente, M. Montminy, A fasting inducible switch modulates gluconeogenesis via activator/coactivator exchange, Nature 456(7219) (2008) 269-73.

[259] J.W. Hwang, H. Yao, S. Caito, I.K. Sundar, I. Rahman, Redox regulation of SIRT1 in inflammation and cellular senescence, Free radical biology \& medicine 61 (2013) 95-110.

[260] Y. Ido, Diabetic complications within the context of aging: Nicotinamide adenine dinucleotide redox, insulin C-peptide, sirtuin 1-liver kinase B1-adenosine monophosphate-activated protein kinase positive feedback and forkhead box O3, J Diabetes Investig 7(4) (2016) 448-58.

[261] J. Lee, A. Padhye, A. Sharma, G. Song, J. Miao, Y.Y. Mo, L. Wang, J.K. Kemper, A pathway involving farnesoid $\mathrm{X}$ receptor and small heterodimer partner positively regulates hepatic sirtuin 1 levels via microRNA-34a inhibition, The Journal of biological chemistry 285(17) (2010) 12604-11. [262] S.E. Choi, T. Fu, S. Seok, D.H. Kim, E. Yu, K.W. Lee, Y. Kang, X. Li, B. Kemper, J.K. Kemper, Elevated microRNA-34a in obesity reduces NAD+ levels and SIRT1 activity by directly targeting NAMPT, Aging Cell 12(6) (2013) 1062-72.

[263] M. Yamakuchi, M. Ferlito, C.J. Lowenstein, miR-34a repression of SIRT1 regulates apoptosis, Proceedings of the National Academy of Sciences of the United States of America 105(36) (2008) $13421-6$.

[264] M. Yamakuchi, C.J. Lowenstein, MiR-34, SIRT1 and p53: the feedback loop, Cell Cycle 8(5) (2009) $712-5$.

[265] J. Lee, J.K. Kemper, Controlling SIRT1 expression by microRNAs in health and metabolic disease, Aging (Albany NY) 2(8) (2010) 527-34.

[266] S. Imai, C.M. Armstrong, M. Kaeberlein, L. Guarente, Transcriptional silencing and longevity protein Sir2 is an NAD-dependent histone deacetylase, Nature 403(6771) (2000) 795-800.

[267] N. Braidy, G.J. Guillemin, H. Mansour, T. Chan-Ling, A. Poljak, R. Grant, Age related changes in NAD+ metabolism oxidative stress and Sirt1 activity in wistar rats, PloS one 6(4) (2011) e19194. 
[268] M. Fulco, Y. Cen, P. Zhao, E.P. Hoffman, M.W. McBurney, A.A. Sauve, V. Sartorelli, Glucose restriction inhibits skeletal myoblast differentiation by activating SIRT1 through AMPK-mediated regulation of Nampt, Developmental cell 14(5) (2008) 661-73.

[269] P. Bai, C. Canto, H. Oudart, A. Brunyanszki, Y. Cen, C. Thomas, H. Yamamoto, A. Huber, B. Kiss, R.H. Houtkooper, K. Schoonjans, V. Schreiber, A.A. Sauve, J. Menissier-de Murcia, J. Auwerx, PARP-1 inhibition increases mitochondrial metabolism through SIRT1 activation, Cell Metab 13(4) (2011) 461-8.

[270] P. Aksoy, C. Escande, T.A. White, M. Thompson, S. Soares, J.C. Benech, E.N. Chini, Regulation of SIRT 1 mediated NAD dependent deacetylation: a novel role for the multifunctional enzyme CD38, Biochemical and biophysical research communications 349(1) (2006) 353-9.

[271] E.J. Kim, J.H. Kho, M.R. Kang, S.J. Um, Active regulator of SIRT1 cooperates with SIRT1 and facilitates suppression of p53 activity, Mol Cell 28(2) (2007) 277-90.

[272] J.E. Kim, J. Chen, Z. Lou, DBC1 is a negative regulator of SIRT1, Nature 451(7178) (2008) $583-6$.

[273] X. Han, J. Niu, Y. Zhao, Q. Kong, T. Tong, L. Han, HDAC4 stabilizes SIRT1 via sumoylation SIRT1 to delay cellular senescence, Clin Exp Pharmacol Physiol 43(1) (2016) 41-6.

[274] L. Peng, Z. Yuan, Y. Li, H. Ling, V. Izumi, B. Fang, K. Fukasawa, J. Koomen, J. Chen, E. Seto, Ubiquitinated sirtuin 1 (SIRT1) function is modulated during DNA damage-induced cell death and survival, The Journal of biological chemistry 290(14) (2015) 8904-12.

[275] T. Sasaki, B. Maier, K.D. Koclega, M. Chruszcz, W. Gluba, P.T. Stukenberg, W. Minor, H. Scrable, Phosphorylation regulates SIRT1 function, PloS one 3(12) (2008) e4020.

[276] F. Salomone, I. Barbagallo, J. Godos, V. Lembo, W. Currenti, D. Cinà, R. Avola, N. D'Orazio, F. Morisco, F. Galvano, Silibinin restores NAD+ levels and induces the SIRT1/AMPK pathway in non-alcoholic fatty liver, Nutrients 9(10) (2017) 1086.

[277] J. Cheng, C. Liu, K. Hu, A. Greenberg, D. Wu, L.M. Ausman, M.W. McBurney, X.-D. Wang, Ablation of systemic SIRT1 activity promotes nonalcoholic fatty liver disease by affecting livermesenteric adipose tissue fatty acid mobilization, Biochimica et Biophysica Acta (BBA)-Molecular Basis of Disease 1863(11) (2017) 2783-2790. 
[278] L.-F. Wang, X.-N. Wang, C.-C. Huang, L. Hu, Y.-F. Xiao, X.-H. Guan, Y.-S. Qian, K.-Y. Deng, H.-B. Xin, Inhibition of NAMPT aggravates high fat diet-induced hepatic steatosis in mice through regulating Sirt1/AMPKa/SREBP1 signaling pathway, Lipids in health and disease 16(1) (2017) 82. [279] Y. Wang, K. Zhu, W. Yu, H. Wang, L. Liu, Q. Wu, S. Li, J. Guo, MiR-181b regulates steatosis in nonalcoholic fatty liver disease via targeting SIRT1, Biochemical and biophysical research communications 493(1) (2017) 227-232.

[280] B. Ritz, F. Yu, The effect of ambient carbon monoxide on low birth weight among children born in southern California between 1989 and 1993, Environmental health perspectives 107(1) (1999) 17-25.

[281] P. Lee, B. Ritz, D. Thomas, Maternal Exposure to Carbon Monoxide and Risk of Term Low Birth Weight Among Singleton Births, Between 1997 and 2005 in Denver, Colorado, Epidemiology 19(6) (2008) S121.

[282] K. Wai, O. Mar, S. Kosaka, M. Umemura, C. Watanabe, Prenatal heavy metal exposure and adverse birth outcomes in Myanmar: a birth-cohort study, International journal of environmental research and public health 14(11) (2017) 1339.

[283] S. Sabra, E. Malmqvist, A. Saborit, E. Gratacós, M.D.G. Roig, Heavy metals exposure levels and their correlation with different clinical forms of fetal growth restriction, PloS one 12(10) (2017) e0185645.

[284] S. Shirai, Y. Suzuki, J. Yoshinaga, Y. Mizumoto, Maternal exposure to low-level heavy metals during pregnancy and birth size, Journal of Environmental Science and Health Part A 45(11) (2010) 1468-1474.

[285] I. Al-Saleh, N. Shinwari, A. Mashhour, A. Rabah, Birth outcome measures and maternal exposure to heavy metals (lead, cadmium and mercury) in Saudi Arabian population, International journal of hygiene and environmental health $217(2-3)(2014)$ 205-218.

[286] J.C. Wells, The thrifty phenotype: An adaptation in growth or metabolism?, Am J Hum Biol 23(1) (2011) 65-75.

[287] W. O'Rourke R, Metabolic thrift and the genetic basis of human obesity, Ann Surg 259(4) (2014) 642-8. 
[288] C. Wittcopp, R. Conroy, Metabolic Syndrome in Children and Adolescents, Pediatrics in review 37(5) (2016) 193.

[289] G.M. Agudelo, G. Bedoya, A. Estrada, F.A. Patino, A.M. Munoz, C.M. Velasquez, Variations in the prevalence of metabolic syndrome in adolescents according to different criteria used for diagnosis: which definition should be chosen for this age group?, Metabolic syndrome and related disorders 12(4) (2014) 202-209.

[290] P. Zimmet, K.G.M. Alberti, F. Kaufman, N. Tajima, M. Silink, S. Arslanian, G. Wong, P. Bennett, J. Shaw, S. Caprio, The metabolic syndrome in children and adolescents-an IDF consensus report, Pediatric diabetes 8(5) (2007) 299-306.

[291] E.R. Burns, S.L. Farr, P.P. Howards, Stressful life events experienced by women in the year before their infants' births-United States, 2000-2010, MMWR. Morbidity and mortality weekly report 64(9) (2015) 247.

[292] S.C. Haight, N. Byatt, T.A.M. Simas, C.L. Robbins, J.Y. Ko, Recorded Diagnoses of Depression During Delivery Hospitalizations in the United States, 2000-2015, Obstetrics \& Gynecology 133(6) (2019) 1216-1223.

[293] P.O. McGowan, S.G. Matthews, Prenatal stress, glucocorticoids, and developmental programming of the stress response, Endocrinology 159(1) (2017) 69-82.

[294] D. Poidatz, E. Dos Santos, F. Duval, H. Moindjie, V. Serazin, F. Vialard, P. De Mazancourt, M.-N. Dieudonné, Involvement of estrogen-related receptor- $y$ and mitochondrial content in intrauterine growth restriction and preeclampsia, Fertility and sterility 104(2) (2015) 483-490.

[295] S.R. Thorn, T.R. Regnault, L.D. Brown, P.J. Rozance, J. Keng, M. Roper, R.B. Wilkening, W.W. Hay Jr, J.E. Friedman, Intrauterine growth restriction increases fetal hepatic gluconeogenic capacity and reduces messenger ribonucleic acid translation initiation and nutrient sensing in fetal liver and skeletal muscle, Endocrinology 150(7) (2009) 3021-3030.

[296] M. Palou, T. Priego, J. Sánchez, A. Palou, C. Picó, Metabolic programming of sirtuin 1 (SIRT1) expression by moderate energy restriction during gestation in rats may be related to obesity susceptibility in later life, British Journal of Nutrition 109(4) (2013) 757-764. 
[297] D. Wolfe, M. Gong, G. Han, T.R. Magee, M.G. Ross, M. Desai, Nutrient sensor-mediated programmed nonalcoholic fatty liver disease in low birthweight offspring, American journal of obstetrics and gynecology 207(4) (2012) 308. e1-308. e6.

[298] N.K. MacLennan, S.J. James, S. Melnyk, A. Piroozi, S. Jernigan, J.L. Hsu, S.M. Janke, T.D. Pham, R.H. Lane, Uteroplacental insufficiency alters DNA methylation, one-carbon metabolism, and histone acetylation in IUGR rats, Physiological genomics 18(1) (2004) 43-50.

[299] E.M. Otis, R. Brent, Equivalent ages in mouse and human embryos, The Anatomical record 120(1) (1954) 33-63.

[300] T.P. Patel, D.M. Gullotti, P. Hernandez, W.T. O'Brien, B.P. Capehart, B. Morrison III, C. Bass, J.E. Eberwine, T. Abel, D.F. Meaney, An open-source toolbox for automated phenotyping of mice in behavioral tasks, Frontiers in behavioral neuroscience 8 (2014) 349.

[301] M.M. Bradford, A rapid and sensitive method for the quantitation of microgram quantities of protein utilizing the principle of protein-dye binding, Analytical biochemistry 72 (1976) 248-54.

[302] D. Bates, M. Maechler, B.M. Bolker, S. Walker, Fitting linear mixed-effects models using Ime4. 2015, J Stat Softw. http://arxiv. org/abs/1406.5823 (2015).

[303] R. Lenth, emmeans: Estimated Marginal Means, aka Least-Squares Means, (2019).

[304] S. Holm, A simple sequentially rejective multiple test procedure, Scandinavian journal of statistics (1979) 65-70.

[305] S.S.o.B. Verification, Biochemical verification of tobacco use and cessation, Nicotine \& Tobacco Research 4(2) (2002) 149-159.

[306] A. Tessitore, G. Cicciarelli, F. Del Vecchio, A. Gaggiano, D. Verzella, M. Fischietti, V. Mastroiaco, A. Vetuschi, R. Sferra, R. Barnabei, MicroRNA expression analysis in high fat dietinduced NAFLD-NASH-HCC progression: study on C57BL/6J mice, BMC cancer 16(1) (2016) 3. [307] A. Asgharpour, S.C. Cazanave, T. Pacana, M. Seneshaw, R. Vincent, B.A. Banini, D.P. Kumar, K. Daita, H.-K. Min, F. Mirshahi, A diet-induced animal model of non-alcoholic fatty liver disease and hepatocellular cancer, Journal of hepatology 65(3) (2016) 579-588. 
[308] A.E. Hill-Baskin, M.M. Markiewski, D.A. Buchner, H. Shao, D. DeSantis, G. Hsiao, S. Subramaniam, N.A. Berger, C. Croniger, J.D. Lambris, Diet-induced hepatocellular carcinoma in genetically predisposed mice, Human molecular genetics 18(16) (2009) 2975-2988.

[309] K.T. Velázquez, R.T. Enos, J.E. Bader, A.T. Sougiannis, M.S. Carson, I. Chatzistamou, J.A. Carson, P.S. Nagarkatti, M. Nagarkatti, E.A. Murphy, Prolonged high-fat-diet feeding promotes non-alcoholic fatty liver disease and alters gut microbiota in mice, World journal of hepatology 11(8) (2019) 619.

[310] C.S. Lieber, M.A. Leo, K.M. Mak, Y. Xu, Q. Cao, C. Ren, A. Ponomarenko, L.M. DeCarli, Model of nonalcoholic steatohepatitis, The American journal of clinical nutrition 79(3) (2004) 502 509.

[311] M. Ito, J. Suzuki, S. Tsujioka, M. Sasaki, A. Gomori, T. Shirakura, H. Hirose, M. Ito, A. Ishihara, H. Iwaasa, A. Kanatani, Longitudinal analysis of murine steatohepatitis model induced by chronic exposure to high-fat diet, Hepatology research : the official journal of the Japan Society of Hepatology 37(1) (2007) 50-7.

[312] S. Nishikawa, A. Yasoshima, K. Doi, H. Nakayama, K. Uetsuka, Involvement of sex, strain and age factors in high fat diet-induced obesity in C57BL/6J and BALB/cA mice, Exp Anim 56(4) (2007) 263-72.

[313] A.N. Larcombe, R.E. Foong, L.J. Berry, G.R. Zosky, P.D. Sly, In utero cigarette smoke exposure impairs somatic and lung growth in BALB/c mice, European Respiratory Journal 38(4) (2011) 932-938.

[314] K.F. Meyer, S. Krauss-Etschmann, W. Kooistra, M. Reinders-Luinge, W. Timens, L. Kobzik, T. Plösch, M.N. Hylkema, Prenatal exposure to tobacco smoke sex dependently influences methylation and mRNA levels of the Igf axis in lungs of mouse offspring, American Journal of Physiology-Lung Cellular and Molecular Physiology 312(4) (2017) L542-L555.

[315] B. Hang, A.M. Snijders, Y. Huang, S.F. Schick, P. Wang, Y. Xia, C. Havel, P. Jacob III, N. Benowitz, H. Destaillats, Early exposure to thirdhand cigarette smoke affects body mass and the development of immunity in mice, Scientific reports 7 (2017) 41915. 
[316] A. Haghighi, D.H. Schwartz, M. Abrahamowicz, G.T. Leonard, M. Perron, L. Richer, S. Veillette, D. Gaudet, T. Paus, Z. Pausova, Prenatal exposure to maternal cigarette smoking, amygdala volume, and fat intake in adolescence, JAMA psychiatry 70(1) (2013) 98-105.

[317] K.W. Lee, M. Abrahamowicz, G.T. Leonard, L. Richer, M. Perron, S. Veillette, E. Reischl, L. Bouchard, D. Gaudet, T. Paus, Prenatal exposure to cigarette smoke interacts with OPRM1 to modulate dietary preference for fat, Journal of psychiatry \& neuroscience: JPN 40(1) (2015) 38.

[318] R.D. Eiden, J. Zhao, M. Casey, S. Shisler, P. Schuetze, C.R. Colder, Pre-and postnatal tobacco and cannabis exposure and child behavior problems: bidirectional associations, joint effects, and sex differences, Drug and alcohol dependence 185 (2018) 82-92.

[319] S.K. Sobrian, L. Marr, K. Ressman, Prenatal cocaine and/or nicotine exposure produces depression and anxiety in aging rats, Progress in Neuro-Psychopharmacology and Biological Psychiatry 27(3) (2003) 501-518.

[320] M.D. Cornelius, L. Goldschmidt, N.M. De Genna, C. Larkby, Long-term effects of prenatal cigarette smoke exposure on behavior dysregulation among 14-year-old offspring of teenage mothers, Maternal and child health journal 16(3) (2012) 694-705.

[321] C. Yochum, S. Doherty-Lyon, C. Hoffman, M.M. Hossain, J.T. Zelikoff, J.R. Richardson, Prenatal cigarette smoke exposure causes hyperactivity and aggressive behavior: role of altered catecholamines and BDNF, Experimental neurology 254 (2014) 145-152.

[322] J.R. Pauly, J.A. Sparks, K.F. Hauser, T.H. Pauly, In utero nicotine exposure causes persistent, gender-dependant changes in locomotor activity and sensitivity to nicotine in C57BI/6 mice, International Journal of Developmental Neuroscience 22(5-6) (2004) 329-337.

[323] M.K. Hasan, T.C. Friedman, C. Sims, D.L. Lee, J. Espinoza-Derout, A. Ume, V. Chalfant, M.L. Lee, I. Sinha-Hikim, K. Lutfy, a 7-Nicotinic Acetylcholine Receptor Agonist Ameliorates Nicotine Plus High-Fat Diet-Induced Hepatic Steatosis in Male Mice by Inhibiting Oxidative Stress and Stimulating AMPK Signaling, Endocrinology 159(2) (2017) 931-944.

[324] A.R. Iskandar, C. Liu, D.E. Smith, K.-Q. Hu, S.-W. Choi, L.M. Ausman, X.-D. Wang, ßCryptoxanthin restores nicotine-reduced lung SIRT1 to normal levels and inhibits nicotine- 
promoted lung tumorigenesis and emphysema in A/J mice, Cancer Prevention Research 6(4) (2013) 309-320.

[325] J. Espinoza-Derout, X.M. Shao, E. Bankole, K.M. Hasan, N. Mtume, Y. Liu, A.P. Sinha-Hikim, T.C. Friedman, Hepatic DNA Damage Induced by Electronic Cigarette Exposure Is Associated With the Modulation of NAD+/PARP1/SIRT1 Axis, Frontiers in Endocrinology 10 (2019). [326] J.C. Kleinman, A. Kopstein, Smoking during pregnancy, 1967-80, American Journal of Public Health 77(7) (1987) 823-825.

[327] J.A. Martin, B.E. Hamilton, S.J. Ventura, F. Menacker, M.M. Park, P.D. Sutton, Births: final data for 2000, (2002).

[328] V.T. Tong, P.M. Dietz, B. Morrow, D.V. D’Angelo, S.L. Farr, K.M. Rockhill, L.J. England, Trends in smoking before, during, and after pregnancy_Pregnancy Risk Assessment Monitoring System, United States, 40 sites, 2000-2010, Morbidity and Mortality Weekly Report: Surveillance Summaries 62(6) (2013) 1-19.

[329] V.T. Tong, P.M. Dietz, S.L. Farr, D.V. D'angelo, L.J. England, Estimates of smoking before and during pregnancy, and smoking cessation during pregnancy: comparing two population-based data sources, Public health reports 128(3) (2013) 179-188.

[330] R.E. Howland, C. Mulready-Ward, A.M. Madsen, J. Sackoff, M. Nyland-Funke, J.M. Bombard, V.T. Tong, Reliability of reported maternal smoking: comparing the birth certificate to maternal worksheets and prenatal and hospital medical records, New York City and Vermont, 2009, Maternal and child health journal 19(9) (2015) 1916-1924.

[331] R.E. Neal, J. Chen, C. Webb, K. Stocke, C. Gambrell, R.M. Greene, M.M. Pisano, Developmental cigarette smoke exposure II: Hepatic proteome profiles in 6 month old adult offspring, Reproductive Toxicology 65 (2016) 414-424.

[332] D. Frescas, L. Valenti, D. Accili, Nuclear trapping of the forkhead transcription factor FoxO1 via Sirt-dependent deacetylation promotes expression of glucogenetic genes, The Journal of biological chemistry 280(21) (2005) 20589-95. 
[333] J.T. Rodgers, C. Lerin, W. Haas, S.P. Gygi, B.M. Spiegelman, P. Puigserver, Nutrient control of glucose homeostasis through a complex of PGC-1alpha and SIRT1, Nature 434(7029) (2005) $113-8$.

[334] L. Zhang, W. Yao, J. Xia, T. Wang, F. Huang, Glucagon-induced acetylation of energysensing factors in control of hepatic metabolism, International journal of molecular sciences $20(8)$ (2019) 1885.

[335] A. Purushotham, T.T. Schug, Q. Xu, S. Surapureddi, X. Guo, X. Li, Hepatocyte-specific deletion of SIRT1 alters fatty acid metabolism and results in hepatic steatosis and inflammation, Cell metabolism 9(4) (2009) 327-338.

[336] R.-H. Wang, C. Li, C.-X. Deng, Liver steatosis and increased ChREBP expression in mice carrying a liver specific SIRT1 null mutation under a normal feeding condition, International journal of biological sciences 6(7) (2010) 682.

[337] A.K. Walker, F. Yang, K. Jiang, J.-Y. Ji, J.L. Watts, A. Purushotham, O. Boss, M.L. Hirsch, S. Ribich, J.J. Smith, Conserved role of SIRT1 orthologs in fasting-dependent inhibition of the lipid/cholesterol regulator SREBP, Genes \& development 24(13) (2010) 1403-1417.

[338] S.v. Buuren, K. Groothuis-Oudshoorn, mice: Multivariate imputation by chained equations in $R$, Journal of statistical software (2010) 1-68.

[339] M. Buler, U. Andersson, J. Hakkola, Who watches the watchmen? Regulation of the expression and activity of sirtuins, The FASEB Journal 30(12) (2016) 3942-3960.

[340] Y. Wu, X. Liu, Q. Zhou, C. Huang, X. Meng, F. Xu, J. Li, Silent information regulator 1 (SIRT1) ameliorates liver fibrosis via promoting activated stellate cell apoptosis and reversion, Toxicology and applied pharmacology 289(2) (2015) 163-76.

[341] Y. Chen, X. Chen, J. Gao, C. Xu, P. Xu, Y. Li, Y. Zhu, C. Yu, Long noncoding RNA FLRL2 alleviated nonalcoholic fatty liver disease through Arntl-Sirt1 pathway, The FASEB Journal (2019) fj. 201900643RRR.

[342] A.J. Drake, P.J. O'Shaughnessy, S. Bhattacharya, A. Monteiro, D. Kerrigan, S. Goetz, A. Raab, S.M. Rhind, K.D. Sinclair, A.A. Meharg, In utero exposure to cigarette chemicals induces 
sex-specific disruption of one-carbon metabolism and DNA methylation in the human fetal liver, BMC medicine 13(1) (2015) 18.

[343] P. Filis, N. Nagrath, M. Fraser, D.C. Hay, J.P. Iredale, P. O'Shaughnessy, P.A. Fowler, Maternal smoking dysregulates protein expression in second trimester human fetal livers in a sexspecific manner, The Journal of Clinical Endocrinology \& Metabolism 100(6) (2015) E861-E870. [344] P.J. O'Shaughnessy, A. Monteiro, S. Bhattacharya, P.A. Fowler, Maternal smoking and fetal sex significantly affect metabolic enzyme expression in the human fetal liver, The Journal of Clinical Endocrinology \& Metabolism 96(9) (2011) 2851-2860.

[345] M.F. Diniz, V.A. Dourado, M.E. Silva, M.L. Pedrosa, F.S. Bezerra, W.G.d. Lima, Cigarette smoke causes changes in liver and spleen of mice newborn exposed during pregnancy, (2013). [346] P. Corbier, D. Edwards, J. Roffi, The neonatal testosterone surge: a comparative study, Archives internationales de physiologie, de biochimie et de biophysique 100(2) (1992) 127-131. [347] S. Pang, F. Tang, Sex differences in the serum concentrations of testosterone in mice and hamsters during their critical periods of neural sexual differentiation, Journal of Endocrinology 100(1) (1984) 7-11.

[348] M. Baum, T. Brand, M. Ooms, J. Vreeburg, A. Slob, Immediate postnatal rise in whole body androgen content in male rats: correlation with increased testicular content and reduced body clearance of testosterone, Biology of reproduction 38(5) (1988) 980-986.

[349] J. Weisz, I.L. Ward, Plasma testosterone and progesterone titers of pregnant rats, their male and female fetuses, and neonatal offspring, Endocrinology 106(1) (1980) 306-316.

[350] A.P. Muraro, R.M.V. Gonçalves-Silva, N.F. Moreira, M.G. Ferreira, A.L. Nunes-Freitas, Y. Abreu-Villaça, R. Sichieri, Effect of tobacco smoke exposure during pregnancy and preschool age on growth from birth to adolescence: a cohort study, BMC pediatrics 14(1) (2014) 99.

[351] J. Martínez-Mesa, A.M. Menezes, D.A. González, B.L. Horta, A. Matijasevich, D.P. Gigante, P.C. Hallal, Life course association of maternal smoking during pregnancy and offspring's height: data from the 1993 Pelotas (Brazil) birth cohort, Journal of Adolescent Health 51(6) (2012) S53S57. 
[352] A.P. Muraro, R.M.V. Gonçalves-Silva, M.G. Ferreira, R. Sichieri, Effect of the exposure to maternal smoking during pregnancy and childhood on the body mass index until adolescence, Revista de saude publica 49 (2015) 41.

[353] A.H. Ziyab, W. Karmaus, R.J. Kurukulaaratchy, H. Zhang, S.H. Arshad, Developmental trajectories of Body Mass Index from infancy to 18 years of age: prenatal determinants and health consequences, J Epidemiol Community Health 68(10) (2014) 934-941.

[354] B. Poljsak, NAMPT-mediated NAD biosynthesis as the internal timing mechanism: In NAD+ World, time is running in its own way, Rejuvenation research 21(3) (2018) 210-224.

[355] S.T. Bond, K.F. Howlett, G.M. Kowalski, S. Mason, T. Connor, A. Cooper, V. Streltsov, C.R. Bruce, K.R. Walder, S.L. McGee, Lysine post-translational modification of glyceraldehyde-3phosphate dehydrogenase regulates hepatic and systemic metabolism, The FASEB Journal 31(6) (2017) 2592-2602.

[356] M. Sokolović, A. Sokolović, D. Wehkamp, E.V.L. van Themaat, D.R. de Waart, L.A. GilhuijsPederson, Y. Nikolsky, A.H. van Kampen, T.B. Hakvoort, W.H. Lamers, The transcriptomic signature of fasting murine liver, BMC genomics 9(1) (2008) 528.

[357] P. Wu, Regulation of the activity of the pyruvate dehydrogenase complex, Advances in enzyme regulation 42 (2002) 249.

[358] Y. Wang, J. Viscarra, S.-J. Kim, H.S. Sul, Transcriptional regulation of hepatic lipogenesis, Nature reviews Molecular cell biology 16(11) (2015) 678.

[359] J.W. Perfield, L.C. Ortinau, R.T. Pickering, M.L. Ruebel, G.M. Meers, R.S. Rector, Altered hepatic lipid metabolism contributes to nonalcoholic fatty liver disease in leptin-deficient $\mathrm{Ob} / \mathrm{Ob}$ mice, Journal of obesity 2013 (2013).

[360] T. Kakuma, Y. Lee, M. Higa, Z.-w. Wang, W. Pan, I. Shimomura, R.H. Unger, Leptin, troglitazone, and the expression of sterol regulatory element binding proteins in liver and pancreatic islets, Proceedings of the National Academy of Sciences 97(15) (2000) 8536-8541.

[361] M. Chełchowska, J. Ambroszkiewicz, J. Mazur, L. Lewandowski, T. Maciejewski, M. Ołtarzewski, J. Gajewski, Effect of tobacco smoking on the maternal and fetal adipokine axis in relation to newborn birth weight and length, Przeglad lekarski 71(11) (2014) 567-571. 
[362] C.S. Mantzoros, A. Varvarigou, V.G. Kaklamani, N.G. Beratis, J.S. Flier, Effect of birth weight and maternal smoking on cord blood leptin concentrations of full-term and preterm newborns, The Journal of Clinical Endocrinology \& Metabolism 82(9) (1997) 2856-2861.

[363] T. Fu, Y.C. Kim, S. Byun, D.H. Kim, S. Seok, K. Suino-Powell, H.E. Xu, B. Kemper, J.K. Kemper, FXR Primes the Liver for Intestinal FGF15 Signaling by Transient Induction of beta-Klotho, Mol Endocrinol 30(1) (2016) 92-103.

[364] S. Kir, S.A. Beddow, V.T. Samuel, P. Miller, S.F. Previs, K. Suino-Powell, H.E. Xu, G.I. Shulman, S.A. Kliewer, D.J. Mangelsdorf, FGF19 as a postprandial, insulin-independent activator of hepatic protein and glycogen synthesis, Science (New York, N.Y.) 331(6024) (2011) 1621-1624. [365] F. Pietrocola, L. Galluzzi, J.M. Bravo-San Pedro, F. Madeo, G. Kroemer, Acetyl coenzyme A: a central metabolite and second messenger, Cell metabolism 21(6) (2015) 805-821.

[366] S. Heinz-Partington, G. Condous, M. Mongelli, Differential effects of cigarette smoking on birth weight by maternal body mass index, Journal of Obstetrics and Gynaecology 36(5) (2016) 608-610.

[367] W. Zheng, K. Suzuki, T. Tanaka, M. Kohama, Z. Yamagata, O.C.H.S. Group, Association between maternal smoking during pregnancy and low birthweight: effects by maternal age, PloS one 11(1) (2016) e0146241.

[368] T. Mine, T. Tanaka, T. Nakasone, T. Itokazu, Z. Yamagata, Y. Nishiwaki, Maternal smoking during pregnancy and rapid weight gain from birth to early infancy, Journal of epidemiology $27(3)$ (2017) 112-116.

[369] X. Wu, J. Huang, L. Dai, J. Zhou, Z. Huang, B. Yu, Adolescent diabetes induced by multiple parental exposures to cigarette smoke condensate, Toxicology letters (2019).

[370] S.E. Purisch, C. Gyamfi-Bannerman, Epidemiology of preterm birth, Seminars in perinatology, Elsevier, 2017, pp. 387-391.

[371] W.J. Simpson, A preliminary report on cigarette smoking and the incidence of prematurity, American journal of obstetrics and gynecology 73(4) (1957) 808-815.

[372] S. Soneji, H. Beltrán-Sánchez, Association of Maternal Cigarette Smoking and Smoking Cessation With Preterm Birth, JAMA network open 2(4) (2019) e192514-e192514. 
[373] H. Goldstein, I.D. Goldberg, T.M. Frazier, G.E. Davis, Cigarette smoking and prematurity, Public Health Reports 79(7) (1964) 553.

[374] N.R. Shah, M.B. Bracken, A systematic review and meta-analysis of prospective studies on the association between maternal cigarette smoking and preterm delivery, American journal of obstetrics and gynecology 182(2) (2000) 465-472.

[375] I.D. Mclntosh, Smoking and pregnancy: II. Offspring risks, Public Health Rev 12(1) (1984) 29 63.

[376] G. Salmasi, R. Grady, J. Jones, S.D. McDonald, K.S. Group*, Environmental tobacco smoke exposure and perinatal outcomes: a systematic review and meta-analyses, Acta obstetricia et gynecologica Scandinavica 89(4) (2010) 423-441.

[377] M. Kharrazi, G.N. DeLorenze, F.L. Kaufman, B. Eskenazi, J.T. Bernert Jr, S. Graham, M. Pearl, J. Pirkle, Environmental tobacco smoke and pregnancy outcome, Epidemiology (2004) 660670.

[378] G. Fantuzzi, G. Aggazzotti, E. Righi, F. Facchinetti, E. Bertucci, S. Kanitz, F. Barbone, G. Sansebastiano, M.A. Battaglia, V. Leoni, Preterm delivery and exposure to active and passive smoking during pregnancy: a case-control study from Italy, Paediatric and perinatal epidemiology $21(3)(2007)$ 194-200.

[379] E. Davis, F. Waffarn, C. Uy, C. Hobel, L. Glynn, C. Sandman, Effect of prenatal glucocorticoid treatment on size at birth among infants born at term gestation, Journal of Perinatology 29(11) (2009) 731.

[380] E. Bevilacqua, R. Brunelli, M.M. Anceschi, Review and meta-analysis: benefits and risks of multiple courses of antenatal corticosteroids, The Journal of Maternal-Fetal \& Neonatal Medicine 23(4) (2010) 244-260.

[381] T. Braun, D.M. Sloboda, B. Tutschek, T. Harder, J.R. Challis, J.W. Dudenhausen, A. Plagemann, W. Henrich, Fetal and neonatal outcomes after term and preterm delivery following betamethasone administration, International Journal of Gynecology \& Obstetrics 130(1) (2015) 6469. 
[382] S.A. Stout, E.V. Espel, C.A. Sandman, L.M. Glynn, E.P. Davis, Fetal programming of children's obesity risk, Psychoneuroendocrinology 53 (2015) 29-39.

[383] E. Turola, S. Petta, E. Vanni, F. Milosa, L. Valenti, R. Critelli, L. Miele, L. Maccio, V. Calvaruso, A.L. Fracanzani, Ovarian senescence increases liver fibrosis in humans and zebrafish with steatosis, Disease models \& mechanisms 8(9) (2015) 1037-1046.

[384] J.S. Klair, J.D. Yang, M.F. Abdelmalek, C.D. Guy, R.M. Gill, K. Yates, A. Unalp-Arida, J.E. Lavine, J.M. Clark, A.M. Diehl, A longer duration of estrogen deficiency increases fibrosis risk among postmenopausal women with nonalcoholic fatty liver disease, Hepatology 64(1) (2016) 8591.

[385] Y. Kamada, S. Kiso, Y. Yoshida, N. Chatani, T. Kizu, M. Hamano, M. Tsubakio, T. Takemura, H. Ezaki, N. Hayashi, Estrogen deficiency worsens steatohepatitis in mice fed high-fat and highcholesterol diet, American Journal of Physiology-Gastrointestinal and Liver Physiology 301(6) (2011) G1031-G1043.

[386] K. Marinou, M. Adiels, L. Hodson, K.N. Frayn, F. Karpe, B.A. Fielding, Young women partition fatty acids towards ketone body production rather than VLDL-TAG synthesis, compared with young men, British journal of nutrition 105(6) (2011) 857-865.

[387] N.I.o. Health, Smoking and Tobacco Control Monograph No. 10: Health effects of exposure to environmental tobacco smoke: The report of the California Environmental Protection Agency: US Department of Health and Human Services, NIH publication, 1999.

[388] S. Gong, Y.-L. Miao, G.-Z. Jiao, M.-J. Sun, H. Li, J. Lin, M.-J. Luo, J.-H. Tan, Dynamics and correlation of serum cortisol and corticosterone under different physiological or stressful conditions in mice, PloS one 10(2) (2015) e0117503.

[389] J.M. Hopkinson, R.J. Schanler, J.K. Fraley, C. Garza, Milk production by mothers of premature infants: influence of cigarette smoking, Pediatrics 90(6) (1992) 934-938.

[390] B.L. Horta, M.S. Kramer, R.W. Platt, Maternal smoking and the risk of early weaning: a metaanalysis, American Journal of Public Health 91(2) (2001) 304.

[391] A. Knudsen, H. Pedersen, J. Klebe, Impact of smoking on the duration of breastfeeding in mothers with insulin-dependent diabetes mellitus, Acta Paediatrica 90(8) (2001) 926-930. 
[392] P. Bachour, R. Yafawi, F. Jaber, E. Choueiri, Z. Abdel-Razzak, Effects of smoking, mother's age, body mass index, and parity number on lipid, protein, and secretory immunoglobulin $A$ concentrations of human milk, Breastfeeding Medicine 7(3) (2012) 179-188.

[393] A. Baheiraei, A. Shamsi, S. Khaghani, S. Shams, M. Chamari, H. Boushehri, A. Khedri, The effects of maternal passive smoking on maternal milk lipid, Acta Medica Iranica (2014) 280-285.

[394] A. Szlagatys-Sidorkiewicz, D. Martysiak-Żurowska, G. Krzykowski, M. Zagierski, B. Kamińska, Maternal smoking modulates fatty acid profile of breast milk, Acta Paediatrica 102(8) (2013) e353-e359.

[395] S.-M. Kim, S.-J. Kim, J.-Y. Kim, J.-R. Kim, K.-H. Cho, Breast Milk from Smokers Contains Less Cholesterol and Protein and Smaller Size of Apolipoprotein Al Resulting in Lower Zebrafish Embryo Survivability, Breastfeeding Medicine 12(6) (2017) 365-372.

[396] A. Winiarska-Mieczan, Cadmium, lead, copper and zinc in breast milk in Poland, Biological trace element research 157(1) (2014) 36-44.

[397] E. Rahimi, M. Hashemi, Z.T. Baghbadorani, Determination of cadmium and lead in human milk, International Journal of Environmental Science \& Technology 6(4) (2009) 671-676.

[398] M. Bassil, F. Daou, H. Hassan, O. Yamani, J.A. Kharma, Z. Attieh, J. Elaridi, Lead, cadmium and arsenic in human milk and their socio-demographic and lifestyle determinants in Lebanon, Chemosphere 191 (2018) 911-921.

[399] M. Zagierski, A. Szlagatys-Sidorkiewicz, A. Jankowska, G. Krzykowski, M. Korzon, B. Kaminska, Maternal smoking decreases antioxidative status of human breast milk, Journal of Perinatology 32(8) (2012) 593.

[400] H.S. Klonoff-Cohen, S.L. Edelstein, E.S. Lefkowitz, I.P. Srinivasan, D. Kaegi, J.C. Chang, K.J. Wiley, The effect of passive smoking and tobacco exposure through breast milk on sudden infant death syndrome, Jama 273(10) (1995) 795-798.

[401] H. Guedes, L. Souza, Exposure to maternal smoking in the first year of life interferes in breastfeeding protective effect against the onset of respiratory allergy from birth to $5 \mathrm{yr}$, Pediatric Allergy and Immunology 20(1) (2009) 30-34. 
[402] H. El Marroun, M.N. Schmidt, I.H. Franken, V.W. Jaddoe, A. Hofman, A. Van Der Lugt, F.C. Verhulst, H. Tiemeier, T. White, Prenatal tobacco exposure and brain morphology: a prospective study in young children, Neuropsychopharmacology 39(4) (2014) 792.

[403] S.J. Roza, B.O. Verburg, V.W. Jaddoe, A. Hofman, J.P. Mackenbach, E.A. Steegers, J.C. Witteman, F.C. Verhulst, H. Tiemeier, Effects of maternal smoking in pregnancy on prenatal brain development. The Generation R Study, European Journal of Neuroscience 25(3) (2007) 611-617. [404] P. Lozano, E. Arillo-Santillán, I. Barrientos-Gutíerrez, L.M. Reynales Shigematsu, J.F. Thrasher, E-cigarette social norms and risk perceptions among susceptible adolescents in a country that bans e-cigarettes, Health Education \& Behavior 46(2) (2019) 275-285. 


\section{CURRICULUM VITAE}

Kendall S. Stocke

University of Louisville School of Public Health and Information Sciences

Department of Environmental and Occupational Health Sciences

Louisville, KY 40202

\section{Education}

$\mathrm{PhD}$

University of Louisville, School of Public Health and Information Technology, Department of Environmental and Occupational Health

M.S.

University of Louisville, Department of Biology Graduated

Postgraduate University of Louisville School of Medicine 2010

Left program before completion to pursue other career options

BS

Kentucky Wesleyan College, Biology Graduated Valedictorian

BS
Aug. 2013-Present

Jan. 2012-August 2013

Aug. 2008-September

May 2008

May 2008

\section{Awards and Honors}

ResearchLouisville! - 1st place poster

September 2014

Alpha Chi National College Honor Scholarship Society

April 2006-May 2008

The National College Dean's List

March 2005

Dean's List and President's Scholar

December 2004

Kentucky Wesleyan College James Graham Brown Scholar

August 2004

\section{Professional Experience}

September 2014-Present

Graduate Research Assistant, University of Louisville, School of Public Health and Information Science, Department of Environmental and Occupational Health, Louisville, KY, Advisor: Dr. Rachel Neal 
February 2014-August 2014

Graduate Research Assistant, University of Louisville, School of Public Health and Information Science, Department of Environmental and Occupational Health, Louisville, KY, Advisor: Dr. Gary Hoyle

August 2013-January 2014

Graduate Research Assistant, University of Louisville, School of Public Health and Information Science, Department of Environmental and Occupational Health, Louisville, KY, Advisor: Dr.

Rachel Neal

April 2012-August 2013

Master's Student, University of Louisville, Department of Biology, Louisville, KY, Advisor: Dr. Cynthia Corbitt

June 2008-August 2008

Research Assistant in Summer Research Scholar Program, Mitchell Memorial Cancer Center, Owensboro, KY, Advisor: Dr. Keith Davis

June 2007-August 2007

Research Assistant in Summer Undergraduate Research Experience, Indiana University School of Medicine Evansville Campus, Advisor: Dr. Tracy Anthony

\section{Areas of Expertise}

Skilled in a variety of laboratory techniques including:

- Western Blot analysis

- PCR

- Inhalation exposure (cigarette smoke, e-cigarette smoke)

- Drug administration

- DEXA scanning for body compositional traits in mice

- Histology (dissection of tissue, tissue processing, paraffin embedding, sectioning of both paraffin and frozen sections, and staining)

- Immunofluorescence

- Laser Capture Microdissection

- Microarray

- Cell line culturing

Skilled in a variety of advanced statistical analyses and bioinformatic techniques including:

- $\quad 16 S$ sequencing analysis (utilizing QIIME and other related tools)

- R statistical programming language (my primary toolset for statistical analyses)

- Python programming language (can write scripts for relatively simple bioinformatics problems)

- Use of Linux and Shell scripting

- Various machine learning techniques and feature engineering (Random Forests, PLSDA, among others)

- Linear and non-Linear Mixed Effects Modeling

- Imputation of missing data

- Basic skill in pulling data from SQL-based databases

\section{Publications}


S. Musah, J. Chen, C. Schlueter, D.M. Humphrey Jr, K. Stocke, M.I. Hoyle, G.W. Hoyle, Inhibition of chlorine-induced airway fibrosis by budesonide, Toxicology and Applied

Pharmacology 363 (2019) 11-21.

R.E. Neal, J. Chen, C. Webb, K. Stocke, C. Gambrell, R.M. Greene, M.M. Pisano, Developmental cigarette smoke exposure II: Hepatic proteome profiles in 6 month old adult offspring, Reproductive Toxicology 65 (2016) 414-424.

R.E. Neal, R. Jagadapillai, J. Chen, C. Webb, K. Stocke, R.M. Greene, M.M. Pisano, Developmental cigarette smoke exposure II: Hippocampus proteome and metabolome profiles in adult offspring, Reproductive Toxicology 65 (2016) 436-447.

R.E. Neal, R. Jagadapillai, J. Chen, C.L. Webb, K. Stocke, C. Gambrell, R.M. Greene, M.M. Pisano, Developmental cigarette smoke exposure II: Kidney proteome profile alterations in 6 month old adult offspring, Reproductive Toxicology 65 (2016) 425-435.

V.L. Massey, K.S. Stocke, R.H. Schmidt, M. Tan, N. Ajami, R.E. Neal, J.F. Petrosino, S. Barve, G.E. Arteel, Oligofructose protects against arsenic-induced liver injury in a model of environment/obesity interaction, Toxicology and Applied Pharmacology 284(3) (2015) 304-314.

\section{Meeting Abstracts}

K. Stocke, C.L. Webb, C. Corbitt, R.M. Greene, M.M. Pisano, R.E. Neal (2019) Sex-dependent Impact of Prenatal Cigarette Smoke Exposure on Phenotypic Characteristics and Hepatic SIRT1 Protein Expression. Ohio Valley Chapter of SETAC (Mar).

IL Burciaga, K Stocke, C Corbitt, RE Neal (2019) Prenatal cigarette exposure and effect on liver function. KAS Annual Meeting, Berea KY (Nov) and Summer Undergraduate Research Program Day (August).

M Kinard, K Stocke, RE Neal, C Corbitt (2019) Prenatal cigarette smoke exposure: neurological and behavioral effects. KAS Annual Meeting, Berea KY (Nov) and Undergraduate Research Day (April).

R. Singhal, H. Donde, S. Ghare, J. Zhang, S. Reddy, S. Joshi-Barve, C. McClain, S. Barve, K. Stocke, M. Vital (2018) Metagenomic analysis identifies that loss of butyrate producing bacteria is a major pathogenic component in alcohol induced gut microbial dysbiosis. Research!Louisville (Oct)

R. Singhal, H. Donde, K. Stocke, S. Ghare, G. Wilson, J. Zhang, S. Joshi-Barve, C. McClain, S. Barve (2018) Alcohol-induced gut microbial dysbiosis is characterized by the loss of butyrate producing bacteria: Relevance to the pathogenesis of alcoholic liver disease (ALD). Research!Louisville (Oct)

K.S. Stocke, C.L. Webb, N. Ajami, R.M. Greene, M.M. Pisano, R.E. Neal (2017) Prenatal Cigarette Smoke Exposure Influences the Cecal Microbiome in Adult Offspring. Keystone Symposia Conference: Bile Acid Receptors as Signal Integrators in Liver and Metabolism (Mar)

K.S. Stocke, N. Ajami. J.F. Petrosino, R.E. Neal (2015) High-Fat Diet Modulation of Fecal Microbiota in Offspring Exposed to Prenatal Alcohol. Ohio Valley Chapter Society of Toxicology (Nov)

K. Stocke, N. Ajami, J. Petrosino, R. Neal (2015) High-Fat Diet Modulation of Fecal Microbiota in Offspring Exposed to Prenatal Alcohol. Research!Louisville (Oct). 
K. Stocke, K.R. Jala, B. Abomoleak, C. Gambrell, R.E. Neal (2014) Gestational Alcohol

Exposure Alters Offspring Fecal Microbial Community Structure. Ohio Valley Chapter Society of Toxicology (Sept)

K. Stocke, K. Jala, B. Abomoleak, C. Gambrell, R. Neal (2014) Gestational Alcohol Exposure Alters Offspring Fecal Microbial Community Structure. Research!Louisville (Sept) K.S. won first place in the School of Public Health (Basic Research)

Bamji, S, R. Page, A. Sanders, D. Patel, A. Alvarez, C. Gambrell, K. Naik, A. Raghavan, K. Stocke, E Gordon, M.E. Burow, S.M. Boue, C.M. Klinge, M. Ivanova, and C. Corbitt (2013) Effects of soy glyceollins on gene expression in mice. KAS (Nov).

\section{Platform Presentations}

K. Stocke, C.L. Webb, N. Ajami, J.F. Petrosino, R.M. Greene, M.M. Pisano, R.E. Neal (2017) Characterization of the Combined Impact of Prenatal Cigarette Smoke Exposure and Postnatal Diet on Sex-Specific Gut Microbiota Community Function in Adult Offspring. OVSOT

Student/Pos-doc Summer Meeting (July) 